\title{
URSACHEN DER
}

\section{RISSBILDUNG BEI}

\section{DIREKTBESCHICHTUNGEN}

MIT MELAMINHARZ-

IMPRÄGNIERTEN PAPIEREN

\author{
Dissertation \\ zur Erlangung des Doktorgrades (Dr. rer. nat.) \\ der Fakultät für Forstwissenschaften und Waldökologie \\ der Georg-August-Universität Göttingen
}

vorgelegt von

Mark Meder

geboren am 02.12.1986 in Weinheim

Braunschweig, 2020 
1. Gutachter:

2. Gutachter:

3. Gutachter:

Tag der mündlichen Prüfung:
Prof. Dr. Carsten Mai (Universität Göttingen)

Prof. Dr. Kai Zhang (Universität Göttingen)

Dr. Dirk Lukowsky (Fraunhofer WKI)

26.11.2020 


\section{DANKSAGUNG}

Meinem ehemaligen Fachbereich Oberflächentechnologie des Fraunhofer WKI danke ich für die außergewöhnliche Arbeitsatmosphäre, die Kollegialität und die schöne Zeit auch außerhalb der Arbeitszeit. Bei Philipp Schmidt und Dr. Stefan Friebel möchte ich mich zudem für die Gespräche zu den chemischen Untersuchungen bedanken. Dem Fachbereich Holzwerkstoff- und NaturfaserTechnologien danke ich für die Hilfe im Technikum, sowie namentlich bei Richard Deetz und Dr. Peter Meinlschmidt für die Diskussionen und Hilfestellungen. Shaghayegh Ameri danke ich für das mathematische Modell und die Hilfe bei Fragen zu Mechanik.

Für die Finanzierung des Aif-Projekts bin ich dem Bundesministerium für Wirtschaft und Energie dankbar. Bedanken möchte ich mich auch bei den Projektpartnern, insbesondere beim internationalen Verein für technische Holzfragen e.V., surfactor Germany GmbH, Ed. Heckewerth Nachf. GmbH \& Co. KG, Saréco Deutschland GmbH sowie Hans Schmid GmbH \& Co. KG für die großzügige Unterstützung des Projekts.

Meiner Familie danke ich für die Unterstützung während des Studiums, der Promotion und der gesamten restlichen Zeit.

Bei Prof. Dr. Carsten Mai bedanke ich mich für die Unterstützung bei der Promotion und den stets konstruktiven Diskussionen. Dank gilt Dr. André Klüppel von der Universität Göttingen für die DVSMessungen.

Besonderer Dank gilt meinem Betreuer und Mentor Dr. Dirk Lukowsky, bei dem ich mich beginnend mit einer Projektarbeit, fortführend in einer Masterthesis sowie abschließend in der Dissertation immer tiefer in die Materie einarbeiten durfte. Bedanken möchte ich mich insbesondere für die unzähligen Diskussionsrunden, das Sparring und die großartige Betreuung.

Danke! 


\section{INHALTSVERZEICHNIS}

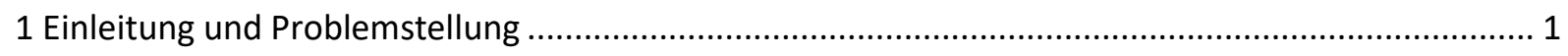

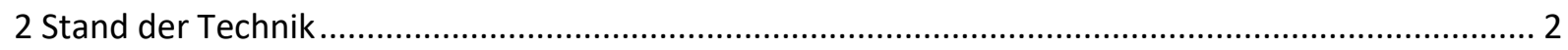

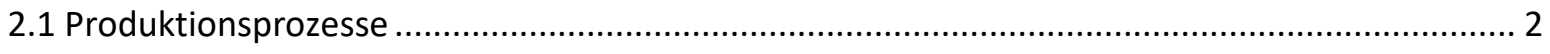

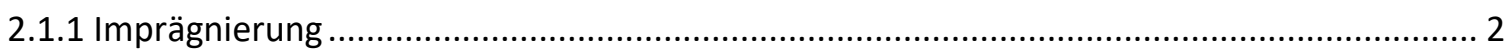

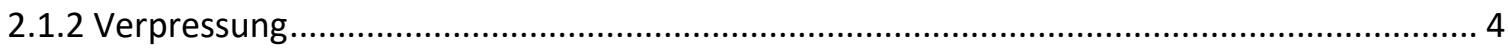

2.2 Aushärtung von Melamin-Imprägnierharzen ................................................................... 5

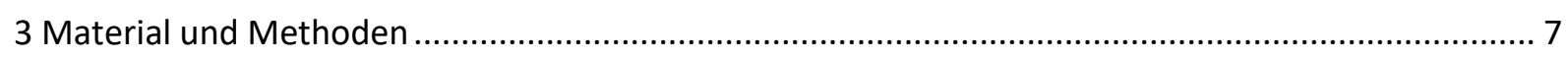

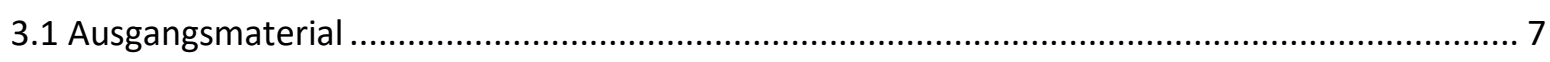

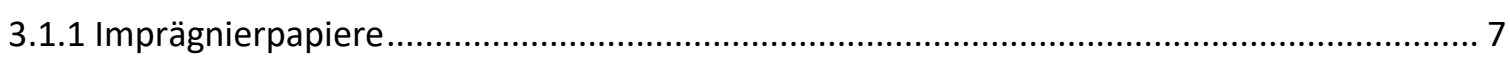

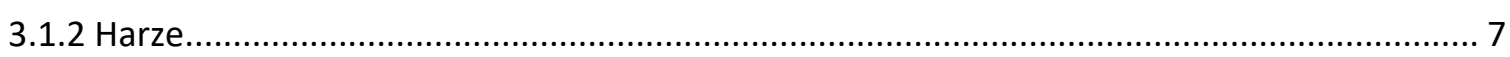

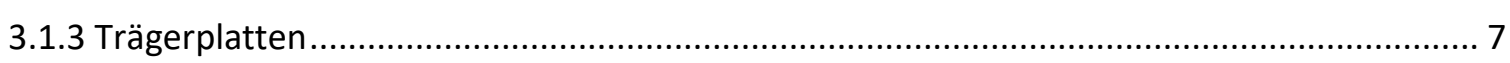

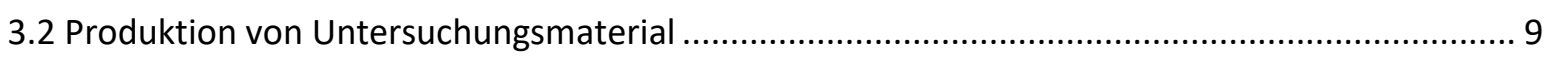

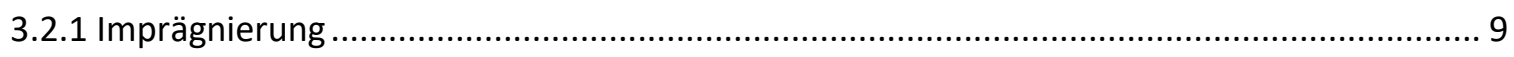

3.2.2 Beschichtungsverfahren und Aushärtung freier Filme................................................... 9

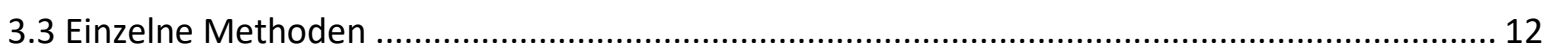

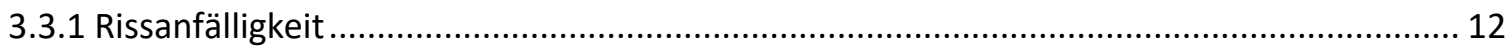

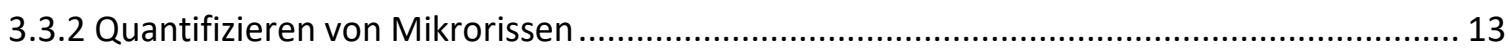

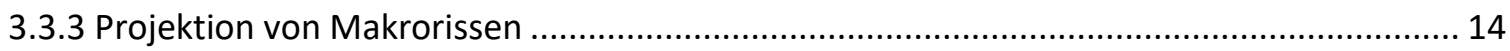

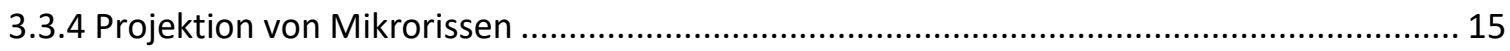

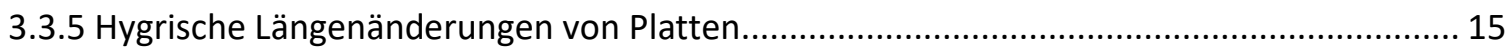

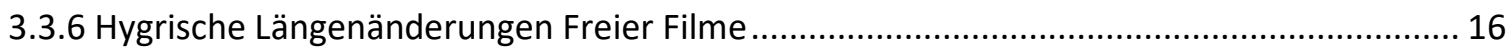

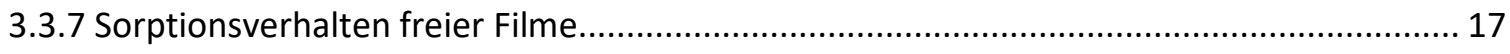

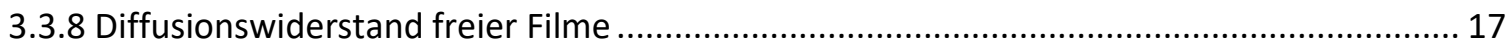

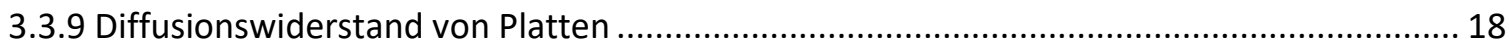

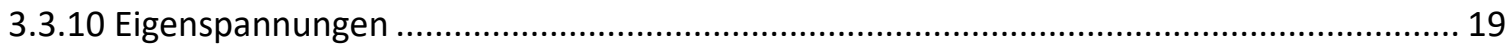

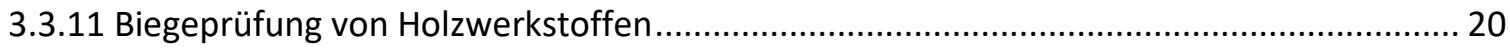

3.3.12 Model zur Berechnung mechanischen Kennwerten der Direktbeschichtung ................... 20

3.3.13 Zugprüfung freier Filme und Imprägnierpapiere ........................................................ 22

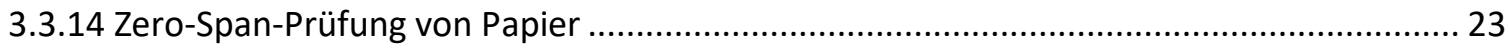

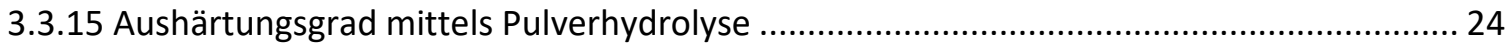

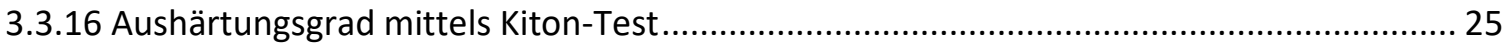

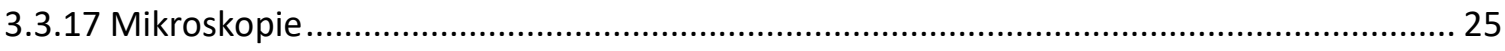

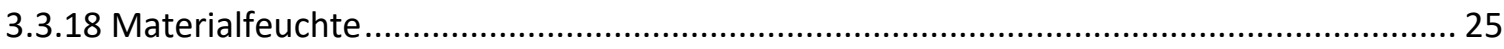

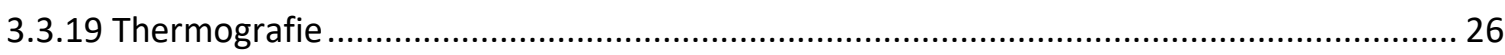

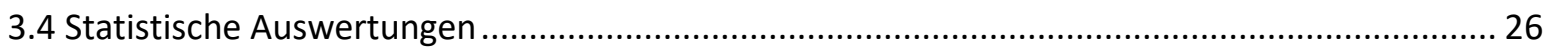




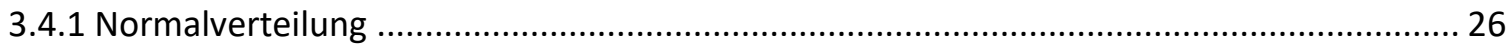

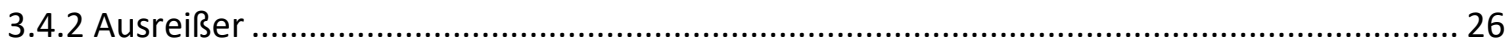

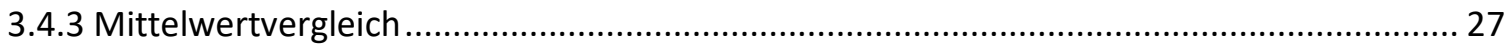

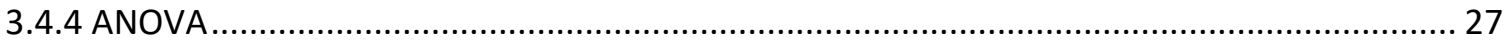

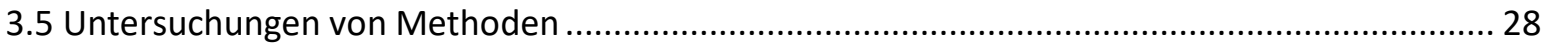

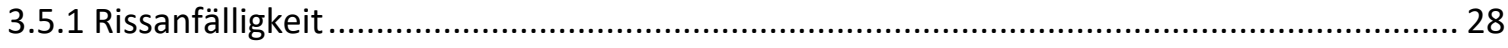

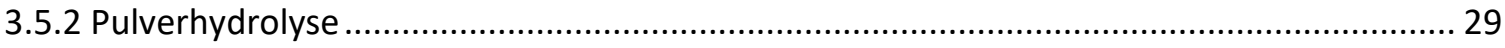

3.5.3 Model zur Berechnung mechanischer Kennwerte der Direktbeschichtung ........................ 30

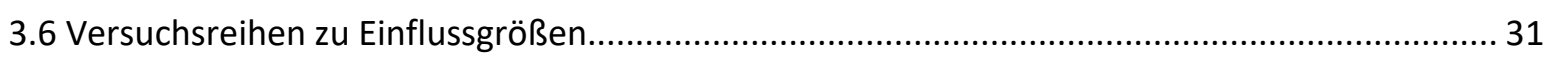

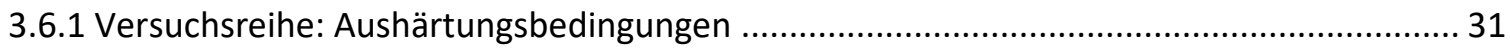

3.6.2 Versuchsreihe: Blättrigkeit und Füllgrad des Papiers......................................................... 32

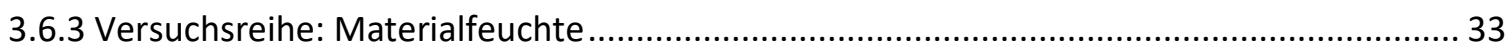

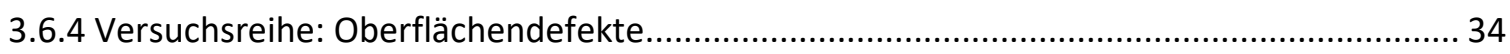

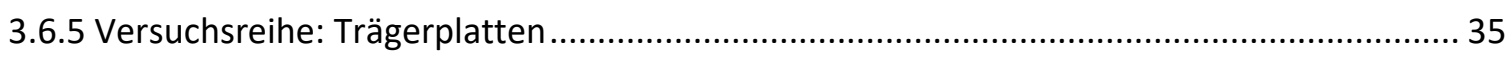

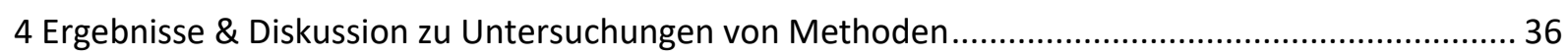

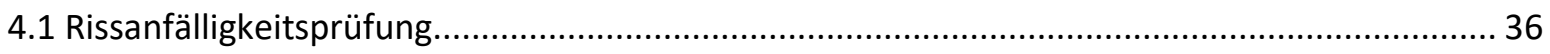

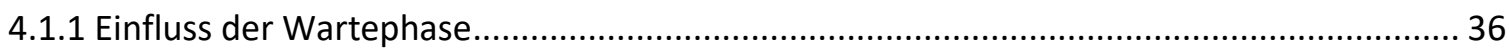

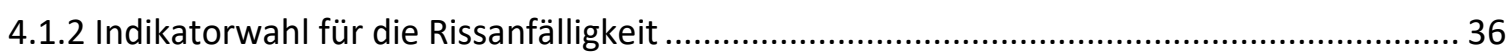

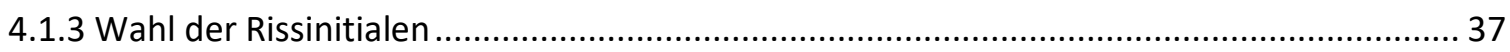

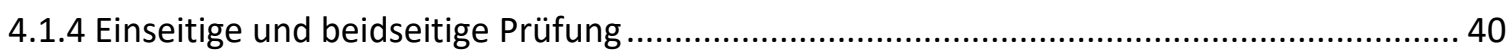

4.1.5 Temperatur im Ofen und Dauer der Ofenbelastung .................................................... 41

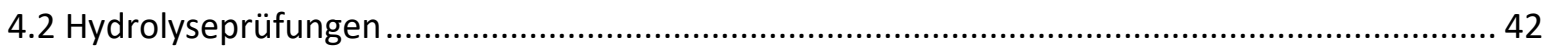

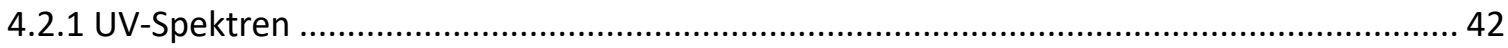

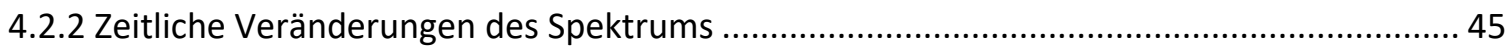

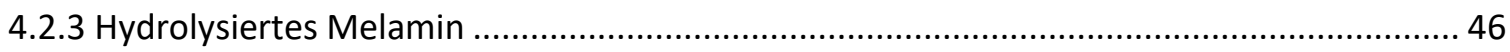

4.2.4 Umgebungstemperatur, Hydrolysedauer und Säurekonzentration .................................. 47

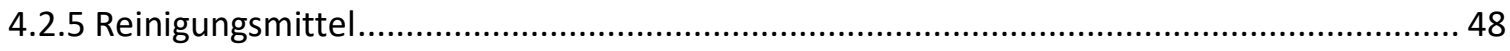

4.2.6 Vergleich des Hydrolyseverfahrens zum Kiton-Test............................................................ 48

4.3 Model zur Berechnung mechanischer Kennwerte der Direktbeschichtung .............................. 50

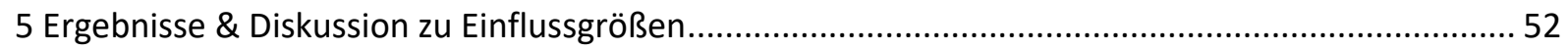

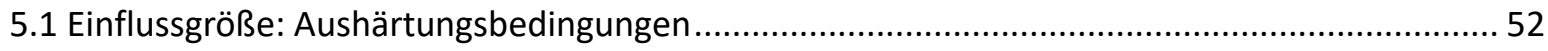

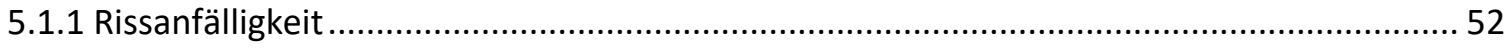

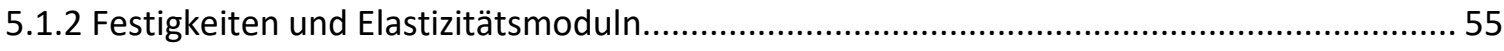

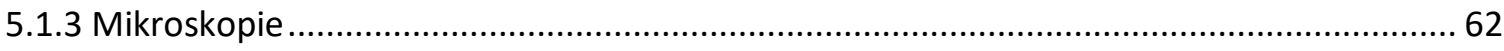

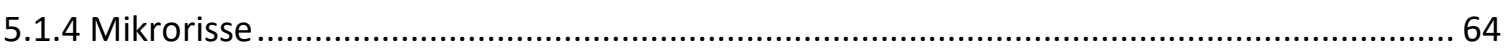

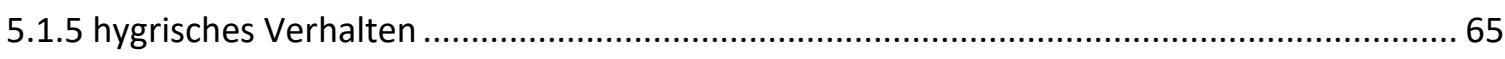




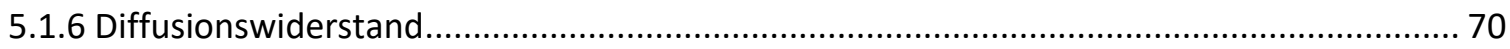

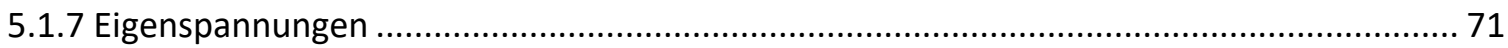

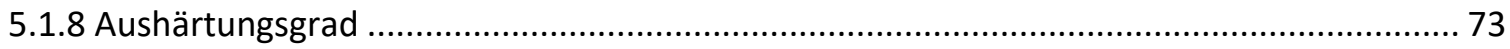

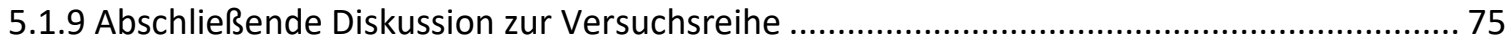

5.2 Einflussgröße: Blättrigkeit und Füllgrad des Papiers ................................................................ 79

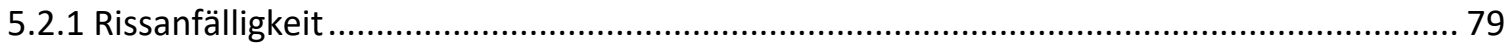

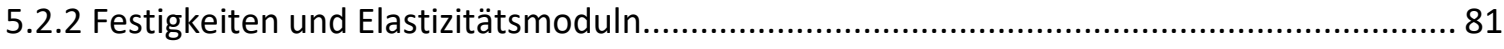

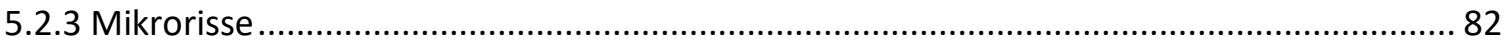

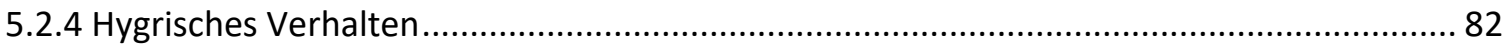

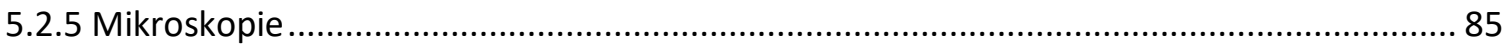

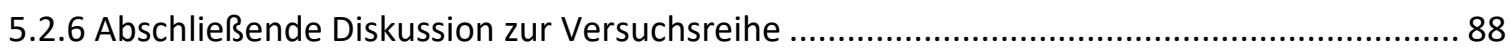

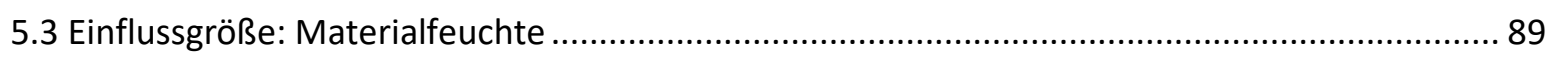

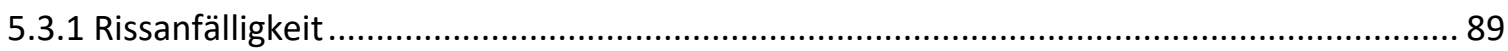

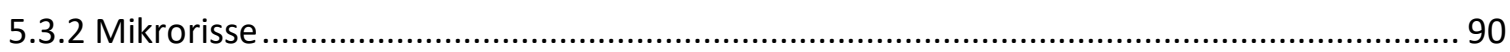

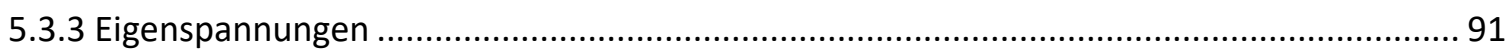

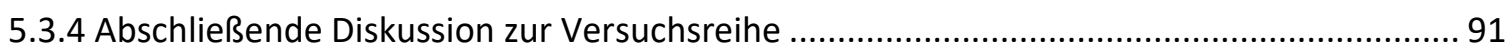

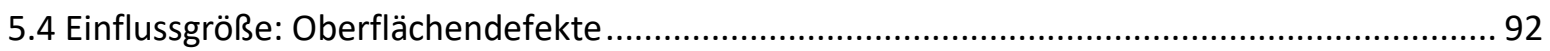

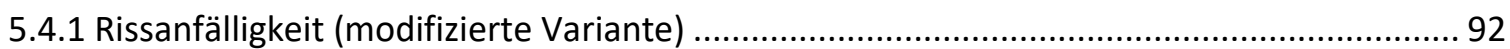

5.4.2 Temperaturverteilung in der Presse................................................................................. 93

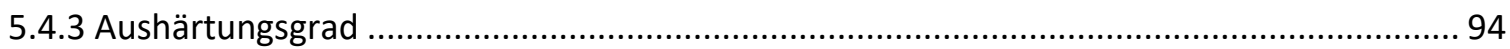

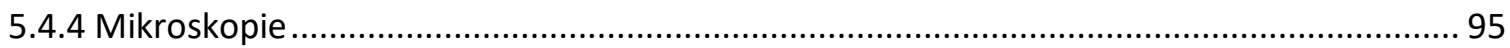

5.4.5 Abschließende Diskussion zur Versuchsreihe ................................................................ 96

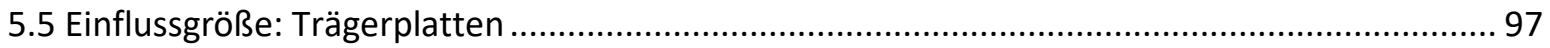

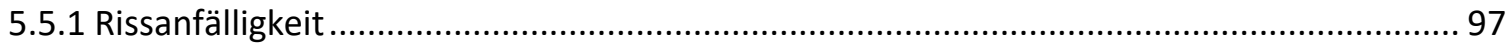

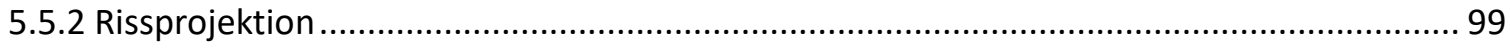

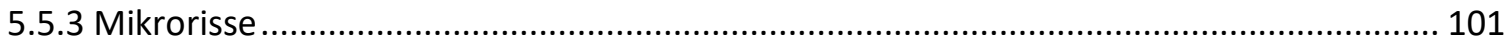

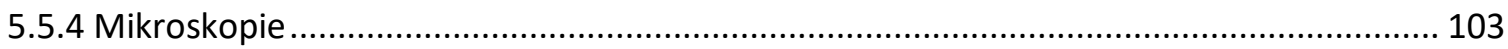

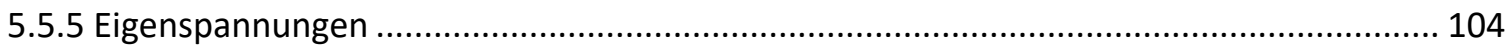

5.5.6 Abschließende Diskussion zur Versuchsreihe .............................................................. 105

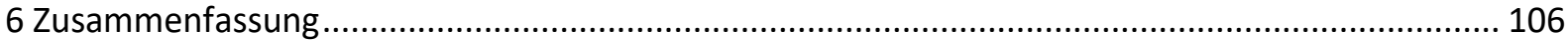

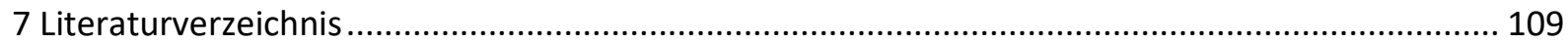




\section{ABBILDUNGSVERZEICHNIS}

Abbildung 1: Schematische Darstellung des ersten Abschnitts einer Imprägnieranlage:

Harzbeaufschlagung...

Abbildung 2: Schematische Darstellung des zweiten Abschnitts einer Imprägnieranlage: Trocknung .. 3 Abbildung 3: Schematische Darstellung des dritten Abschnitts einer Imprägnieranlage: Trocknung.... 4

Abbildung 4: Methylolierung von Melamin nach MerLINE; VUKUSIC; ABDALA 2012 ............................. 5

Abbildung 5: Vernetzung von methyloliertem Melamin nach MERLINE; VUKUSIC; ABDALA 2012 ............. 5

Abbildung 6: Zuschnitt der Papiere nach der Imprägnierung ........................................................ 9

Abbildung 7: Schematischer Stapel der Materialien bei den drei Beschichtungsverfahren................ 10

Abbildung 8: Auf- (links) und Seitenansicht (rechts) für eine Probe zur Messung der Rissanfälligkeit

von Direktbeschichtungen .................................................................................. 12

Abbildung 9: Verschiedene Vergrößerungen einer Platte. ...................................................... 13

Abbildung 10: Arbeitsschritte bei der Rissprojektion. ........................................................... 14

Abbildung 11: Ablauf des Probenzuschnitts freier Filme zur Messung der hygrischen Längenänderung.

Abbildung 12: Produktionsschritte direktbeschichteter Platten zu Prüfkörpern für die Messung von

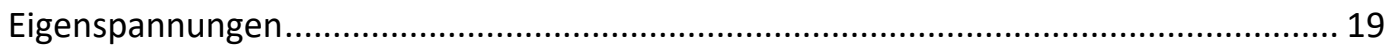

Abbildung 13: Prüfkörper mit Verformung auf Grund von Eigenspannungen. ................................ 19

Abbildung 14: Produktionsschritte vom freien Film bzw. Papieren zu Prüfkörpern für Zugversuche.. 22

Abbildung 15: Hergestelltes Prüfmaterial zur Untersuchung der Auswirkungen von

Aushärtungsbedingungen........................................................................ 31

Abbildung 16: Lage der einzelnen Oberflächendefekte auf den Plattenhälften............................... 34

Abbildung 17: Risse an Bohrlöcher bei der Rissanfälligkeitsprüfung. ........................................... 38

Abbildung 18: Risse an Nuten bei der Rissanfälligkeitsprüfung................................................... 38

Abbildung 19: Gemittelte kumulierte Risslängen (links) und Materialfeuchten (rechts) von

Probensätzen bei Variation von Temperatur und Dauer der Rissanfälligkeitsprüfung.... 41

Abbildung 20: Verlauf der relativen Luftfeuchte verschiedener Ausgangsfeuchten bei Erhöhung der

Temperatur ................................................................................................. 42

Abbildung 21: Strukturformeln von Melamin und dessen Substitutionsprodukte.............................42

Abbildung 22: UV-Spektren von Melamin, Ammelin, Ammelid, Cyanursäure und Harnstoff sowie ein

typisches Spektrum der Pulverhydrolyse einer reinen MF-Direktbeschichtung. ........... 43

Abbildung 23: Extinktionen einer Pulverhydrolyse sowie von Harzbestandteilen. .............................44

Abbildung 24: UV-Spektren derselben Hydrolyselösung direkt nach der Hydrolyse (0 h), nach mehrstündiger Lagerung ( $1 \mathrm{~h}$ bis $96 \mathrm{~h}$ ) und geschüttelt nach 96-stündiger Lagerung.... 45

Abbildung 25: Extinktionen bei $237 \mathrm{~nm}$ von Melamin unterschiedlicher Konzentrationen gelöst in

Salzsäure (15\%-ig)

Abbildung 26: Hydrolysierte Melaminmasse [ $\mu \mathrm{g}$ ] für Hydrolysen durchgeführt bei einer

Umgebungstemperatur von $21^{\circ} \mathrm{C}$ (links) und $40{ }^{\circ} \mathrm{C}$ (rechts) mit Säurekonzentrationen von $5 \%$ bis $20 \%$ und Hydrolysedauern von $1 \mathrm{~h}$ bis $8 \mathrm{~h}$

Abbildung 27: Hydrolysierte Melaminmassen [ $\mu \mathrm{g}$ ] für Hydrolysen von Oberflächen, die vorher auf unterschiedliche Art und Weise gereinigt wurden ................................................48

Abbildung 28: Aushärtungsgrade nach der Pulverhydrolyse und nach dem Kiton-Test ................... 49

Abbildung 29: Sensitivitätsanalyse zum Model...................................................................... 50

Abbildung 30: Kumulierte Risslängen [mm] direktbeschichteter Platten unterschiedlicher

Aushärtungsgrade.

Abbildung 31: Stark überhärtete Probe $(25 \mathrm{~cm} \times 25 \mathrm{~cm})$ mit schnell härtendem Harz nach der Rissanfälligkeitsprüfung. 
Abbildung 32: Gemessene Biegefestigkeiten $\left[\mathrm{N} / \mathrm{mm}^{2}\right]$ und Elastizitätsmoduln $\left[\mathrm{N} / \mathrm{mm}^{2}\right]$ von direktbeschichteten Platten $(n=6)$ sowie berechnete Zugfestigkeiten und Elastizitätsmoduln von Direktbeschichtungen unterschiedlicher Aushärtungsgrade...... 55

Abbildung 33: Schematischer Spannungsverlauf bei der Biegeprüfung ............................................ 56

Abbildung 34: Biegefestigkeiten $\left[\mathrm{N} / \mathrm{mm}^{2}\right]$ und Elastizitätsmoduln $\left[\mathrm{N} / \mathrm{mm}^{2}\right]$ von erneut heißverpressten Spanplatten und einer unverpressten Referenz................................. 57

Abbildung 35: Zugfestigkeiten [N/ $\left.\mathrm{mm}^{2}\right]$ und Elastizitätsmoduln $\left[\mathrm{N} / \mathrm{mm}^{2}\right]$ freier Filme

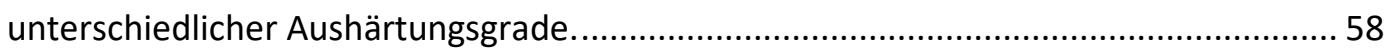

Abbildung 36: Verdrehen freier Filme unterschiedlicher Presstemperatur. ...................................... 59

Abbildung 37: Zero-Span-Festigkeiten $\left[\mathrm{N} / \mathrm{mm}^{2}\right]$ sowie Zug-Festigkeiten $\left[\mathrm{N} / \mathrm{mm}^{2}\right]$ von parallel sowie senkrecht zur Produktionsrichtung geprüften Papieren. .................................................6 60

Abbildung 38: Bruchkanten von Papierproben bei einem Zugversuch (links) und einer Zero-SpanPrüfung (rechts).

Abbildung 39: Risse unterschiedlicher Tiefen an einer stark überhärteten Probe................................ 62

Abbildung 40: Mikroskopische Aufnahmen von Mikrorissen (UV-Fluoreszenz mit Anregung bei $365 \mathrm{~nm}$ ).

Abbildung 41: Mikrorissdichte $\left[\mu \mathrm{m} / \mathrm{cm}^{2}\right]$ direktbeschichteter Platten unterschiedlicher

Aushärtungsgrade. .64

Abbildung 42: Aufnahme einer Probe mit Makrorissen und hoher Mikrorissdichte.

Abbildung 43: Kurven zur hygrischen Längenänderung freier Filme unterschiedlicher

Aushärtungsgrade.

Abbildung 44: Sorption und Desorption [\%] von freien Filmen unterschiedlicher Aushärtungsgrade. 66

Abbildung 45: Kurven zur hygrischen Längenänderung von Spanplatten.

Abbildung 46: Diffusionsverhalten direktbeschichteter Platten und freier Filme.

Abbildung 47: Änderungen des Stichmaßes [mm], wenn die relative Luftfeuchte von $30 \%$ auf $85 \%$ erhöht wird.

Abbildung 48: Durch Pulverhydrolyse gelöstes Melamin [mg/l] direktbeschichteter Platten unterschiedlicher Aushärtungsgrade.

Abbildung 49: Wiederholung der Biegefestigkeiten $\left[\mathrm{N} / \mathrm{mm}^{2}\right]$ direktbeschichteter Platten und Spanplatten, der Zugfestigkeiten [N/ $\mathrm{mm}^{2}$ ] freier Filme als auch der Zero-Span-Festigkeit $\left[\mathrm{N} / \mathrm{mm}^{2}\right]$ der Papiere. 75

Abbildung 50: Wiederholung der Rissanfälligkeit [mm], Biegefestigkeiten $\left[\mathrm{N} / \mathrm{mm}^{2}\right]$ und Mikrorissdichte $\left[\mu \mathrm{m} / \mathrm{cm}^{2}\right]$ derselben direktbeschichteten Platten.

Abbildung 51: Zusammenhang von Biegefestigkeiten direktbeschichteter Platten und deren

Mikrorissdichte

Abbildung 52: Arithmetische Mittelwerte von kumulierten Risslängen [mm] direktbeschichteter

Platten unterschiedlicher Blättrigkeit (links) und unterschiedlicher Papiere (rechts) . ... 79

Abbildung 53: Aufnahme einer 2-Blättrigen Probe nach der Rissanfälligkeitsprüfung. .80

Abbildung 54: Zugfestigkeiten (links) und Elastizitätsmoduln (rechts) 1-blättriger und 2-blättriger freier Filme.

Abbildung 55: Kurven zur hygrischen Längenänderung freier Filme.

Abbildung 56: Sorption und Desorption von 1-blättrigen und 2-blättrigen freien Filmen. 83

Abbildung 57: Aufsicht auf einen Riss bei einer 1-blättrigen Direktbeschichtung (oben) und

Querschnitte von gerissenen 1-blättrigen (Mitte) und 2-blättrigen Proben (unten) ....... 85

Abbildung 58: Dünnschliffe von 1-blättrigen (oben) und 2-blättrigen (unten) Direktbeschichtungen

(links) und freien Filmen (rechts) 86

Abbildung 59: Kumulierte Risslängen [mm] direktbeschichteter Platten unterschiedlicher Materialfeuchten beim Verpressen. 
Abbildung 60: Mikrorisse $\left[\mu \mathrm{m} / \mathrm{cm}^{2}\right]$ direktbeschichteter Platten unterschiedlicher Materialfeuchten beim Verpressen.

Abbildung 61: Stichmaß [mm] beim ariden Klima (links) und Änderungen des Stichmaßes [mm], wenn die relative Luftfeuchte von 30\% auf 85\% erhöht wird (rechts).................................... 91

Abbildung 62: Position der Defektstellen (oben) und kumulierte Risslängen für $5 \mathrm{~cm} \times 5 \mathrm{~cm}$ Raster (unten).

Abbildung 63: Thermografie-Aufnahmen des oberen Pressblechs direkt nach dem Direktbeschichten einer Platte

Abbildung 64: Position der Defektstellen (oben) und Aushärtungsgrade $[\mathrm{mg} / \mathrm{l}]$ an ausgewählten Stellen (unten).

Abbildung 65: Querschnitte (oben) und Aufsichten (unten) über Nuten (rechts) und abseits von Nuten (links)

Abbildung 66: Kumulierte Risslängen [mm] direktbeschichteter Platten unterschiedlicher

Trägerplatten.

Abbildung 67: $25 \mathrm{~cm} \times 25 \mathrm{~cm}$ Rissanfälligkeitsprobe der Grobspanplatte nach der Prüfung. 99 Abbildung 68: Aufnahmen der unteren Hälften von Rissanfälligkeitsproben der Sperrhölzer nach der Prüfung. 100

Abbildung 69: Mikrorissdichten $\left[\mu \mathrm{m} / \mathrm{cm}^{2}\right]$ direktbeschichteter Platten mit unterschiedlichen

Trägerplatten. 101

Abbildung 70: Mikrorissprojektion bei der Grobspanplatte. 101

Abbildung 71: Mikrorissprojektion bei der Grobspanplatte. 102

Abbildung 72: Querschnitte direktbeschichteter Sperrhölzer abseits von Rissen (oben) und an Rissen (unten).

Abbildung 73: Riss in der Grobspanplatte.

Abbildung 74: Änderungen des Stichmaßes [mm], wenn die relative Luftfeuchte von $30 \%$ auf $85 \%$ erhöht wird. 


\section{TABELLENVERZEICHNIS}

Tabelle 1: Herstellerangaben zu den verwendeten Imprägnierpapieren...................................... 7

Tabelle 2: Parameter der verwendeten Trägerplatten. .......................................................... 8

Tabelle 3: Parameter zur Rissanfälligkeitsmethode ............................................................... 12

Tabelle 4: Parameter zur Quantifizierung von Mikrorissen ......................................................... 13

Tabelle 5: Parameter zur Projektion von Makrorissen............................................................. 14

Tabelle 6: Parameter zur Projektion von Mikrorissen.......................................................... 15

Tabelle 7: Parameter zur Messung hygrischer Längenänderung von Platten ................................... 15

Tabelle 8: Parameter zur Messung hygrischer Längenänderung freier Filme ................................. 16

Tabelle 9: Parameter zur Messung des Sorptionsverhaltens freier Filme ...................................... 17

Tabelle 10: Parameter zur Messung des Diffusionswiderstands freier Filme .................................. 17

Tabelle 11: Parameter zur Messung des Diffusionswiderstands von Platten .................................. 18

Tabelle 12: Parameter zur Messung von Eigenspannungen .................................................... 19

Tabelle 13: Parameter zur Biegeprüfung von Holzwerkstoffen .....................................................20

Tabelle 14: Parameter zur Zugprüfung freier Filme und Imprägnierpapiere.................................... 22

Tabelle 15: Parameter zur Zero-Span-Prüfung von Papier.......................................................... 23

Tabelle 16: Parameter zur Messung des Aushärtungsgrades mittels Pulverhydrolyse ...................... 24

Tabelle 17: Parameter zur Messung des Aushärtungsgrades mittels Kiton-Test .............................25

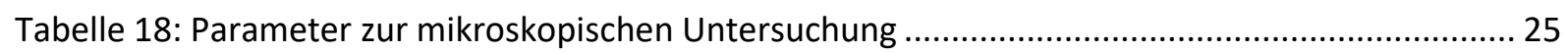

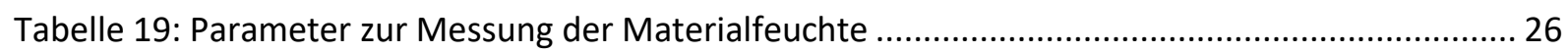

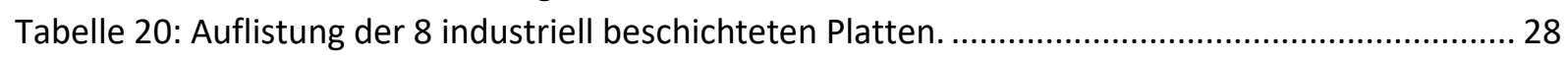

Tabelle 21: Auflistung des hergestellten Untersuchungsmaterials und der daran durchgeführten Untersuchungen zu Aushärtungsbedingungen ................................................. 32

Tabelle 22: Auflistung des hergestellten Untersuchungsmaterials und der daran durchgeführten Untersuchungen zur Blättrigkeit.

Tabelle 23: Auflistung des hergestellten Untersuchungsmaterials und der daran durchgeführten Untersuchungen zu Materialfeuchten

Tabelle 24: Auflistung des hergestellten Untersuchungsmaterials und der daran durchgeführten Untersuchungen zu Oberflächendefekten ....

Tabelle 25: Auflistung des hergestellten Untersuchungsmaterials und der daran durchgeführten

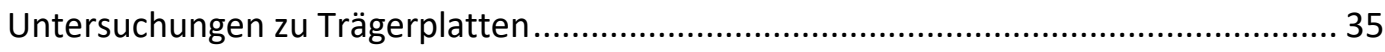

Tabelle 26: Indikatoren als Maß für die Rissanfälligkeit direktbeschichteter Platten ..........................36

Tabelle 27: Kumulierte Risslängen [mm] direktbeschichteter Platten an unterschiedlichen Rissinitialen.

Tabelle 28: Kumulierte Risslänge [mm] direktbeschichteter Platten bei einseitigen und beidseitigen Rissinitialen

Tabelle 29: Biegefestigkeiten $\left[\mathrm{N} / \mathrm{mm}^{2}\right]$ und Elastizitätsmodul $\left[\mathrm{N} / \mathrm{mm}^{2}\right]$ von direktbeschichteten Spanplatten............................................................................................. 56

Tabelle 30: Vergleich von Literaturwerten mit den Messwerten zum hygrischen Quellen freier Filme.

Tabelle 31: Differentielle Quell- und Schwindmaße von Spanplatten

Tabelle 32: Längen- und Massenänderung bei Quellung freier Filme sowie von OEHLER (2016) vermessenen Materialien. 


\section{FORMELVERZEICHNIS}

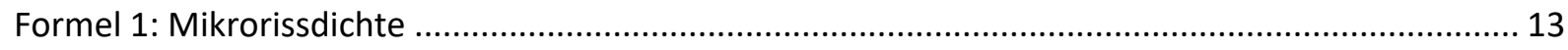

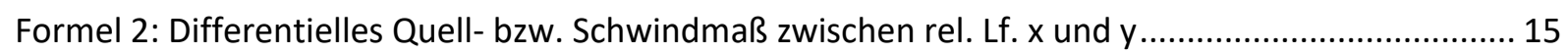

Formel 3: Wasserdampfdiffusionswiderstands-zahl nach DIN EN ISO 12572 (2017) .......................... 18

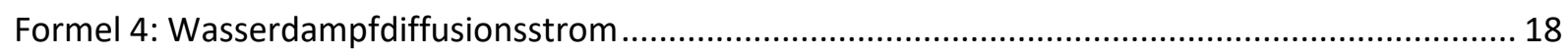

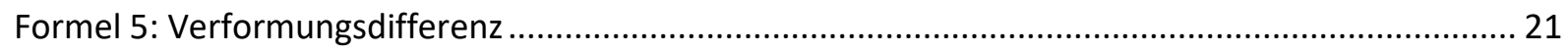

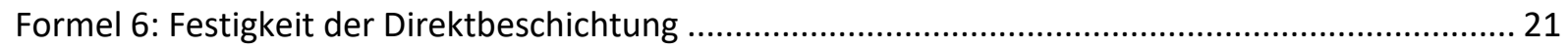

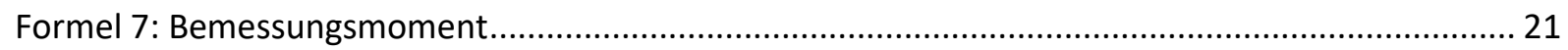

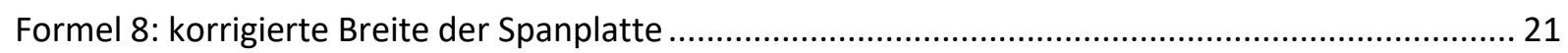

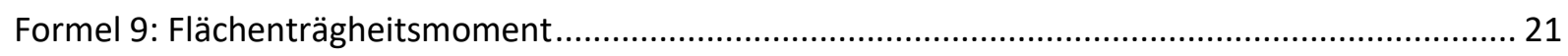

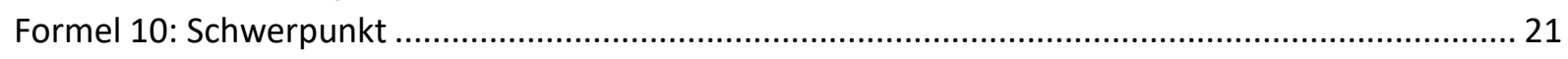

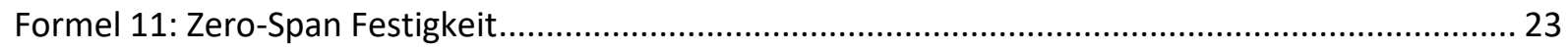

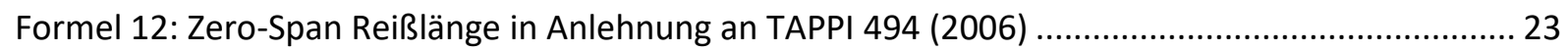

Formel 13: Zero-Span Bruchlast nach Pulmac International (Unbekannt) ......................................... 23

Formel 14: Berechnung der Konzentration hydrolysierten Melamins aus der Extinktion..................... 24

Formel 15: Berechnung der Masse hydrolysierten Melamins aus der Extinktion ................................ 24

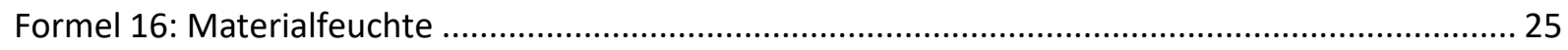

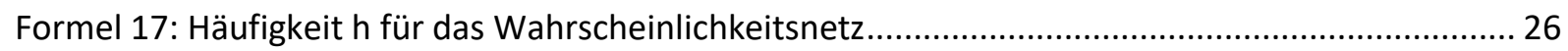

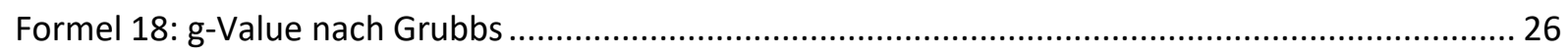

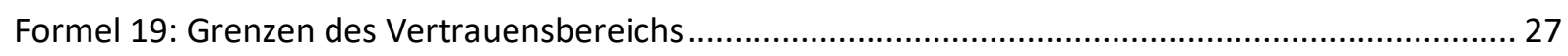

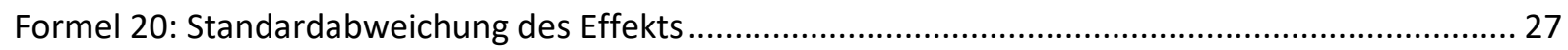

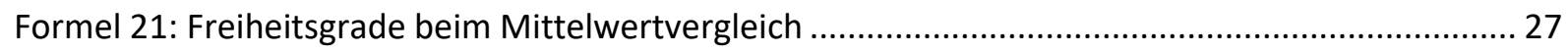

Formel 22: relative Luftfeuchte bei bekannter absoluter Luftfeuchte ............................................. 29

Formel 23: Absolute Feuchte bei bekannter relativer Luftfeuchte....................................................29

Formel 24: Sättigungsdampfdruck für Temperaturen zwischen $-50^{\circ} \mathrm{C}$ bis $+100^{\circ} \mathrm{C}$ (Magnus-Formel) 29 


\section{EINLEITUNG UNd PROBLEMStelLUNG}

Direktbeschichtungen zählen zu den meist verwendeten Holzbeschichtungen für die Innenanwendung (Anonymus 2012; ICDLI 2017). 2013 wurden allein in Deutschland ca. 550 Millionen $\mathrm{m}^{2}$ Direktbeschichtungen gekauft und 850 Millionen $\mathrm{m}^{2}$ dekorative Laminate (sowohl Direktbeschichtungen als auch HPL) produziert. Weltweit werden 2,5 Milliarden $\mathrm{m}^{2}$ papierbasierte Oberflächen jedes Jahr produziert, Tendenz steigend. (Freedonia Group 2014)

Bei Direktbeschichtungen handelt es sich um mit Harz imprägnierte Papiere, welche auf Holzwerkstoffe verpresst werden. Unter Druck und Hitze härtet das Harz aus und wird mit der Trägerplatte quasiverschweißt. Direktbeschichtungen sind mechanisch, thermisch als auch chemisch belastbar (ZEPPENFELD; GRUNWALD 2005). Sie werden daher auch in Bereichen mit hoher Belastung wie Küchen- und Labormobiliar oder Fußböden eingesetzt. Es können jedoch Risse in der Direktbeschichtung auftreten. Wie häufig Risse auftreten ist nicht bekannt. Es handelt sich jedoch nicht um ein vernachlässigbares Problem. Risse, die während der Produktion auftreten, sind weniger problematisch. Solche Risse werden während der Qualitätskontrolle erkannt und die Platten aussortiert. Es werden erfahrungsbasierte Anpassungen in der Produktion vorgenommen und die Probleme abgestellt. Viel problematischer sind Risse, die erst nach Tagen, Wochen oder Monaten auftreten. Wenn die Platten bereits verbaut sind, sind Reklamation oft teuer. Schadenssummen können mehrere Hunderttausend bis Millionen Euro betragen. Von 2012 bis 2015 wurden zehn Schadensanalysen zu gerissenen Direktbeschichtungen am Fraunhofer WKI durchgeführt. Da die Direktbeschichtung untrennbar mit der Trägerplatte quasiverschweißt ist, lassen sich die Ursachen für die Rissbildung oft nicht identifizieren. Forschungsarbeiten zu Rissen in Direktbeschichtungen liegen nicht vor. Vom 01.09.2016 bis 31.08.2018 wurde daher ein Projekt (IGF-Projekt 18693 N) durchgeführt, um Einflussgrößen der Rissbildung zu identifizieren und neue Methoden zur Aufnahme wichtiger Kennwerte zu entwickeln. Im Rahmen des Projekts wurde diese Dissertation angefertigt.

Es werden zahlreiche Synonyme für Direktbeschichtungen genutzt. Teilweise leiten sich diese von Normen ab. Die Bezeichnung „direct pressed laminate“ (DPL) wird von der Norm für Laminatböden (DIN EN 13329:2017) für die Beschichtung verwendet. Dagegen umfasst der Begriff „,melamine faced board“ (MFB) (DIN EN 14323:2014) sowohl die Beschichtung als auch die Trägerplatte. In Australien und Neuseeland werden solche Platten als "reconstituted wood-based panel“ bezeichnet (AS/NZS 4266.0:2004). Andere Bezeichnungen haben ihren Ursprung in den Produktionsprozessen, wie "KT-Platte" für Kurztaktplatte. In den USA werden sie aufgrund des Quasiverschweißens des Melaminharzes mit dem Holzwerkstoff als "thermally fused melamine“ (TFM) bezeichnet. Für die Dissertation wurden folgende Begriffe gewählt:

- „Direktbeschichtung“ für die Beschichtung exklusive der Trägerplatte

- „direktbeschichtete Platte“ für die Beschichtung inklusive der Trägerplatte 


\section{STAND DER TECHNIK}

Im Folgenden sind die Produktionsprozesse von Direktbeschichtungen und die Aushärtung von mit Melaminharz imprägnierten Papieren dargestellt. Zur Rissbildung von Direktbeschichtungen wurden keine wissenschaftlichen Untersuchungen gefunden.

\subsection{PROdUKTIONSPROZESSE}

Die Produktion von direktbeschichteten Platten lässt sich in zwei voneinander entkoppelte Prozessschritte gliedern; Imprägnierung und Direktbeschichtung.

\subsubsection{IMPRÄGNIERUNG}

Bei der Imprägnierung durchläuft das Papier drei Abschnitte: Harzbeaufschlagung, Trocknung und Formatierung. Ziel ist es das Papier mit der wässrigen Harzlösung zu imprägnieren und das Harz soweit vorzukondensieren, dass es sich nicht mehr vom Papier löst.

\section{HARZBEAUFSCHLAGUNG}

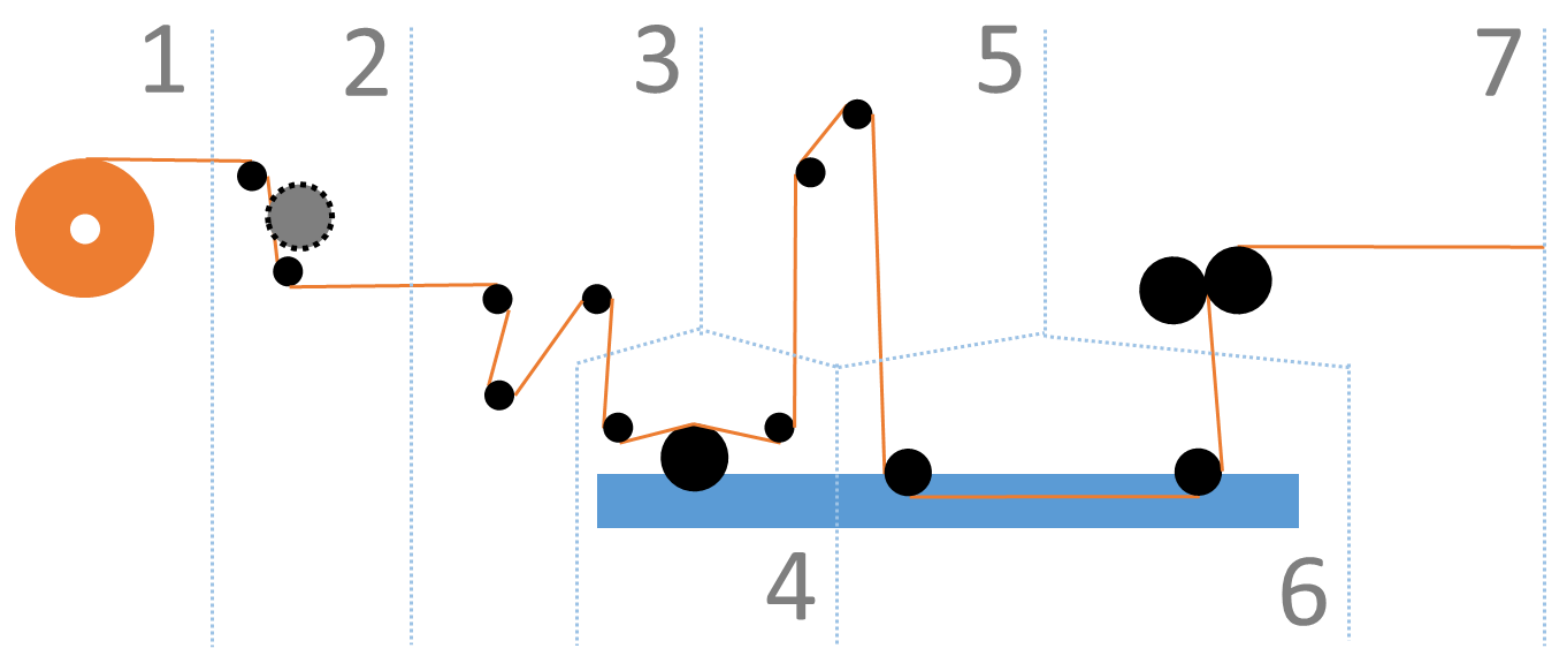

Abbildung 1: Schematische Darstellung des ersten Abschnitts einer Imprägnieranlage: Harzbeaufschlagung

Das zu imprägnierende Papier ist als Rolle eingespannt (Abbildung 1) und wird durch die Imprägnieranlage gezogen. Ein Motor an der Einspannvorrichtung unterstützt den Abspulvorgang (1). Direkt im Anschluss befindet sich ein Besäumer (2). Da sich beim späteren Harzaufschlag und Trocknen die Bahnbreite verändert, findet nur ein grober Zuschnitt statt. Durch den Voreinschnitt auf das grobe Endmaß lässt sich Harz einsparen; es muss weniger Papier imprägniert werden. Allerdings können Einrisse an den Papierrändern entstehen. Zudem führt die verringerte Bahnbreite zu einer höheren Zugspannung im Papier. Das Risiko eines Bahnrisses steigt. Ein Vorbesäumen findet daher nur statt, wenn die Harzersparnis das Risiko eines Bahnrisses überwiegt. Nachfolgend wird das Papier über mehrere Rollen geführt (3). Durch die Lage der Walzen zueinander wird der Anpressdruck des Papiers erhöht. Die Zugspannung, mit der das Papier durch die Maschine geführt wird, wird besser übertragen. Zwei Rollen drücken die Papierbahn auf eine Auftragswalze (4). Die Walze ist zum Teil in eine Harzwanne eingetaucht. Durch die Rotation der Walze wird Harz aufgenommen und auf das Papier übertragen. Durch die einseitige Beaufschlagung kann das Harz in das Papier eindringen und dabei die Luft verdrängen. Würde das Harz beidseitig aufgebracht, könnte die Luft nicht entweichen und würde zu Defekten beim Verpressen führen. Direkt nach der Auftragswalze wird das Papier über die Atemstrecke geführt (5). In dieser Phase soll dem viskosen Harz Zeit gegeben werden, in das Papier 
einzudringen. Je feinporiger das Papier bzw. viskoser das Harz ist, desto länger benötigt es dafür. Die Länge der Atemstrecke wird entsprechend angepasst. Bei der nachfolgenden Tränkung wird das Papier durch eine Harzwanne geführt und nimmt dabei große Mengen des Harzes auf (6). Direkt im Anschluss wird die Papierbahn durch Dosierwalzen geführt (7). Durch den Abstand zwischen den Walzen lässt sich der Harzauftrag einstellen. Verdrängtes Harz fließt in die Wanne zurück.

Derartig imprägnierte Papiere werden als 1-stufig imprägniert bezeichnet. Bei mehrstufigen Imprägnierungen wird die Papierbahn nach den Dosierwalzen an Wärmestrahlern vorbeigeführt. Das Harz kondensiert leicht aus. Danach wird das Papier in eine zweite Harzwanne getaucht und erneut die Auftragsmenge über Dosierwalzen reguliert. Durch eine mehrstufige Imprägnierung wird das Papier mit mehreren Lagen Harz imprägniert, z.B. um den Papierkern mit einem günstigeren Harnstoffharz zu imprägnieren und ihn mit teurerem, hochwertigerem Melaminharz zu ummanteln. Man spricht von Kern- und Mantelharz.

\section{$\underline{\text { TROCKNUNG }}$}

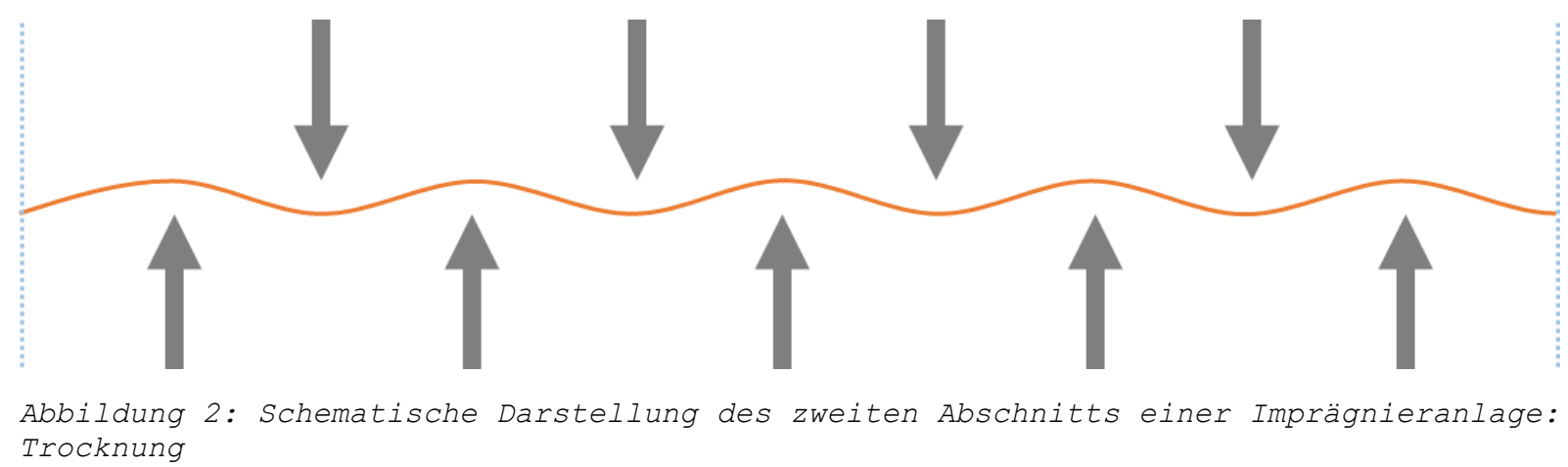

Die Trocknung dient dazu, das flüssige Harz im Papier zu fixieren und das Harz im Papier lagerfähig und handhabbar zu machen. Die imprägnierte Papierbahn (kurz Imprägnat) wird dafür durch einen Trockner geführt. Durch diesen Trockner wird die Bahn nicht mit Walzen, sondern mit Luftströmen geführt. Das Harz kondensiert leicht. Durch diese Polykondensationsreaktion wird Wasser frei, welches vom Luftstrom abgetragen wird. Anhand der Materialfeuchte lässt sich ableiten, wie stark die Vorkondensation ausgefallen ist. Ist die Feuchte niedrig, war die Vorkondensation stark, ist die Feuchte hoch, war sie schwach. Die Trocknung ist ein Nebeneffekt, an dem man den erwünschten Effekt, die Vernetzung des Harzes, beurteilen kann. Ist die Strömungsgeschwindigkeit zu stark oder die Temperatur zu hoch eingestellt, trocknet das Papier ohne die gewünschte Kondensation aus. Die Endfeuchte lässt sich dann nicht mehr als Maß der Kondensation verwenden. 


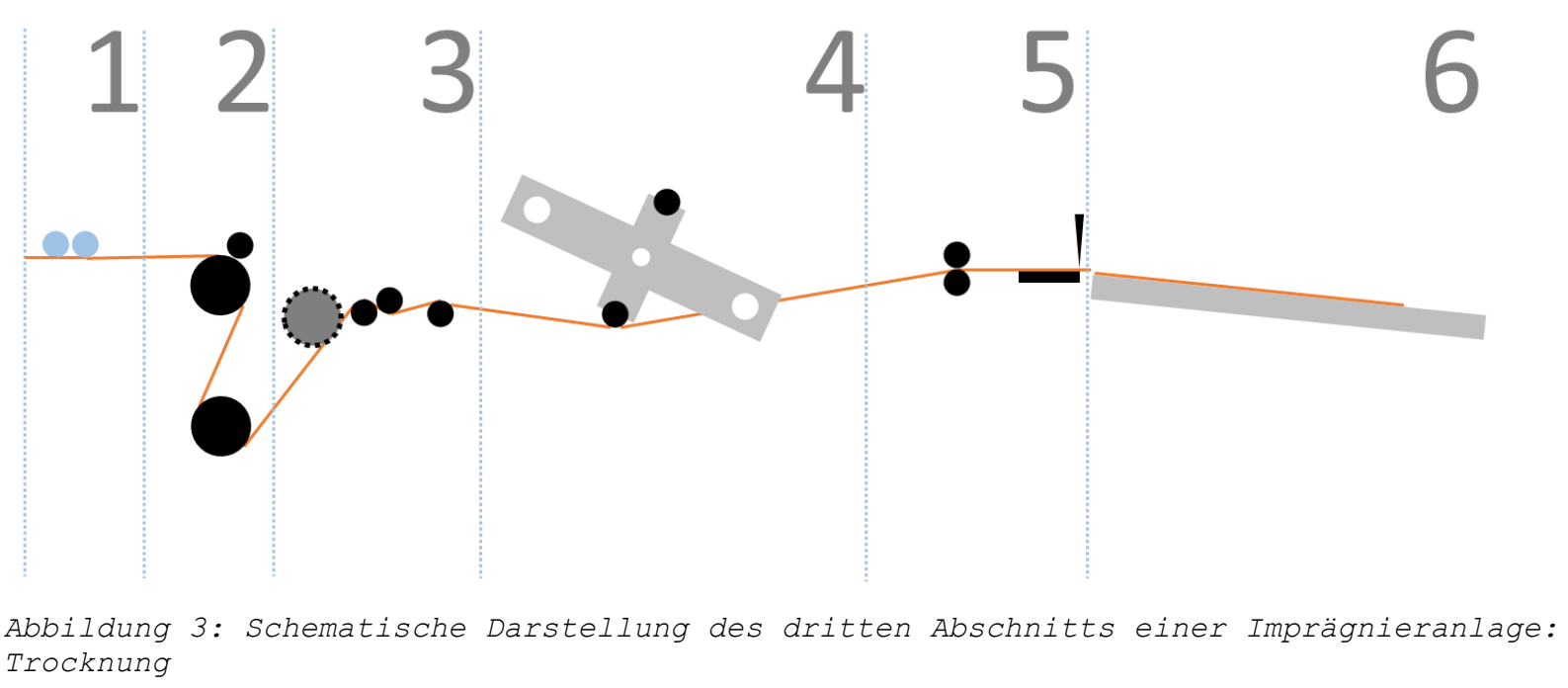

Bei der Formatierung soll das imprägnierte Papier auf die gewünschte Blattgröße bzw. Rollenbreite gebracht werden. Zuerst wird das vom Trocknen aufgewärmte Papier mit Kühlwalzen (1) auf Raumtemperatur gebracht (Abbildung 3). Anschließend wird die Bahn über mehrere Walzen geführt, um erneut den Anpressdruck zu erhöhen und eine bessere Führung zu gewährleisten (2). Die Papierbahn wird auf die gewünschte Endbreite besäumt (3) und aufgerollt. Werden als Endprodukt Bögen gewünscht, wird die Bahn an dem Aufroller (4) vorbei in den Längsformatierer (5) geführt. Dieser schneidet die Bahn auf die gewünschte Länge und die entstehenden Bögen werden auf einem Ablagetisch gesammelt (6).

\subsubsection{VERPRESSUNG}

Ein Stapel aus Imprägnat, Trägerplatte und weiterem Imprägnat wird in eine Taktpresse gefahren und bei ca. $185^{\circ} \mathrm{C}$ und $2,5 \mathrm{~N} / \mathrm{mm}^{2}$ zusammengepresst. Druck und Temperatur werden üblicherweise nicht variiert. Die Dauer der Heißverpressung ist abhängig von der Reaktivität des Harzes und der gewünschten Oberflächengüte. Eine zu geringe Pressdauer kann Offenporigkeit, Haftungsprobleme und geringe Chemikalienresistenz bewirken. Dem gegenüber stehen Kantenausbrüche beim Zuschnitt und eine geringe Verformbarkeit bei zu hoher Pressdauer. Meist wird eine möglichst geringe Pressdauer angestrebt, um den Durchsatz zu erhöhen. Die Temperatur in der Presse wird über die gesamte Pressdauer gehalten. Nach dem Öffnen der Presse wird die beschichtete Platte entnommen und gestapelt oder separiert ausgekühlt.

Die Oberflächenstruktur der Direktbeschichtung lässt sich über die Pressbleche einstellen. Soll eine Hochglanzoberfläche entstehen, werden die Pressbleche nahe Raumtemperatur zurückgekühlt, bevor die Presse öffnet. 


\subsection{AUSHÄRTUNG VON MELAMIN-IMPRÄGNIERHARZEN}

Die Aushärtungsreaktion von Melamin-Formaldehyd-Harzen lässt sich in zwei Schritte gliedern; Methylolierung und Vernetzung (KohlmaYr, et al. 2013; MerLine; et al. 2012). Bei der Methylolierung reagieren die primären Aminogruppen des Melamins mit Formaldehyd zu sekundären und tertiären Aminogruppen (Abbildung 4). Das Molverhältnis von Melamin zu Formaldehyd bestimmt wie viele der Aminogruppen umgesetzt werden. Dieser Reaktionsschritt findet vor der Imprägnierung des Papiers statt.

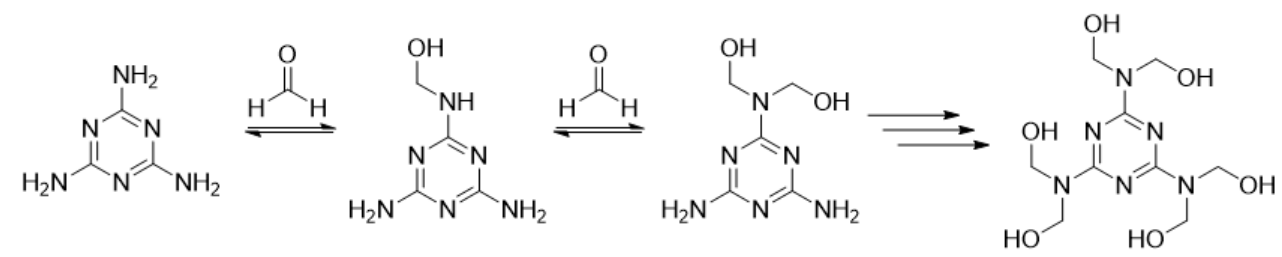

Abbildung 4: Methylolierung von Melamin nach MERLINE; VUKUSIC; ABDALA 2012

Die Vernetzung findet sowohl bei der Imprägnierung als auch der Verpressung statt. Bei der Vernetzung kann sich methyloliertes Melamin über zwei Brücken binden. Zwischen zwei Methylolgruppen können Methylen-Ether-Brücke entstehen (Abbildung 5). Bindet sich eine Methylolgruppe an ein primäres Amin bildet sich eine Methylen-Brücke. In beiden Fällen läuft eine Kondensationsreaktion ab, Wasser wird frei. (KANDELBAUER, et al. 2009a; KoHLMAYR, et al. 2013). Die Vernetzung kann sowohl rein thermisch als auch mit Härtern wie Ammoniumsalze erfolgen (DIEM; MATTHIAS; WAGNER 2000). Die gängige Praxis bei Direktbeschichtungen ist eine Aushärtung mittels Temperierung und Härterzusatz. Dadurch wird ein schnellerer Reaktionsablauf gewährleistet. (Merline; VuKusic; ABdala 2012)<smiles>OCN(CO)c1nc(N(CO)CO)nc(N(CO)CO)n1</smiles><smiles>OCN(CO)c1nc(N(CO)CO)nc(N(CO)CO)n1</smiles><smiles>OCN(CO)c1nc(N(CO)CO)nc(N(CO)COCN(CO)c2nc(N(CO)CO)nc(N(CO)CO)n2)n1</smiles><smiles>OCN(CO)c1nc(N(CO)CO)nc(N(CO)CO)n1</smiles><smiles>Nc1cc(O)nc(N)n1</smiles><smiles>OCN(CO)c1nc(N(CO)CO)nc(N(CO)CN(CO)c2nc(N(CO)CO)nc(N(CO)CO)n2)n1</smiles>
Vernetzung 
Geräte wie der patentierte Golombek-Prüfapparat (GoLomBeK Unbekannt; US 5001068:1991) messen das Aushärteverhalten. Das Harz im Imprägnat härtet unter oszillierenden Bewegungen in einem Ölbad aus. Aus dem Verlauf des Strömungswiderstands des Imprägnats lassen sich Informationen zur Flexibilität, Aushärtungsgeschwindigkeit und Fließzeit ziehen (KANDELBAUER, et al. 2009b).

Studien bescheinigen der Imprägnatsfeuchte bei der Aushärtung eine wichtige Rolle (KOHLMAYR, et al. 2013). Ist das Imprägnat zu feucht, kommt es zum sogenannten Blocken. Gestapelte bzw. aufgerollte Imprägnate kleben zusammen und können nicht mehr verwendet werden. Ist es zu trocken, hat das Imprägnat eine zu geringe Reaktivität. Die Adhäsion zur Trägerplatte fällt mangelhaft aus und Härte, Glanz und Medienresistenz sind geringer. (KANDELBAUER, et al. 2009a)

Zur Bestimmung des Aushärtungsgrads gibt es verschiedene Ansätze. Beim Kiton-Test (AS/NZS 4266.21:2004) werden die Abnahme des Glanzgrads und die Verfärbung auf Grund einer FarbSäure-Lösung evaluiert. Auf welchen Mechanismen diese Reaktion beruht, ist nicht bekannt. Es wird jedoch vermutet, dass die Farblösung an den freien Methylolgruppen reagiert. Je unterhärteter das Harz, desto mehr freie Methylolgruppen sind vorhanden und desto stärker reagiert die Farblösung. Die Industrie nutzt meist eine hauseigene Abwandlung der Norm. GUENTHER (1988) zeigt eine Möglichkeit auf die Shore-Härte zur Aushärtungsbestimmung heranzuziehen. Mittels NIR-Spektroskopie können sowohl von Melaminharzen als auch von Phenolharzen Aushärtungsgrade ermittelt werden (KANDELBAUER, et al. 2010; WASHINGTON, et al. 2009). Dieses online-taugliche Verfahren wird teilweise bereits bei der kontinuierlichen Produktion von Continous Pressed Laminate (kurz CPL) angewendet. Das Patent zur NIR-Messung (EP 3238934 A1:2017) schreibt allerdings eine Kalibrierung mittels Säuremethoden vor. 


\section{Material und Methoden}

\subsection{AUSGANGSMATERIAL}

Das Untersuchungsmaterial wurde zusammen mit Industriepartnern des Forschungsprojekts ausgewählt. Es sollte von den Ausgangskomponenten Papier, Harz und Trägerplatte sowohl eine rissunempfindliche als auch eine rissanfällige Variante verwendet werden. Bei den Papieren wurde dazu der Füllgrad (im Wesentlichen der Titandioxidgehalt) variiert, bei den Harzen die Reaktivität. Als Trägerplatten wurden Spanplatten gewählt, die sich hinsichtlich Recyclinganteil und Größe der Deckschichtspäne unterscheiden. Um den Einfluss der Trägerplatte auf die Rissbildung umfassender untersuchen zu können, wurde die Auswahl um weitere Holzwerkstoffe erweitert.

\subsubsection{IMPRÄGNIERPAPIERE}

Es wurden zwei Imprägnierpapiere der Firma Munksjö Paper GmbH verwendet. Beide Papiere wurden als Rollen angeliefert. Tabelle 1 listet die Herstellerangaben zu den Papieren auf.

Tabelle 1: Herstellerangaben zu den verwendeten Imprägnierpapieren.

Laut Hersteller beschreibt der Aschegehalt den Titandioxidgehalt der Papiere.

\begin{tabular}{l|l|c|c} 
Materialeigenschaft & Prüfmethode & $\begin{array}{l}\text { Gefültes } \\
\text { Standardpapier }\end{array}$ & $\begin{array}{l}\text { Geringer } \\
\text { gefülltes } \\
\text { Alternativpapier }\end{array}$ \\
\hline Flächengewicht $\left[\mathrm{g} / \mathrm{m}^{2}\right]$ & DIN EN ISO 536 (2012) & 75 & 75 \\
Aschegehalt [\%] & ISO $2144(2015)$ & 40 & 30 \\
pH-Wert [] & ISO $6588-2$ (2012) & $6,5-7,5$ & $6,5-7,5$ \\
Luftdurchlässigkeit & ISO $5636-5$ (2013) & $19-20$ & 14 \\
nach Gurley [s] & DIN 53107 (2016) & $100-110$ & 50 \\
Glätte nach Beck [s] & DIN ISO 3781 (2012) & 7 & 9
\end{tabular}

\subsubsection{HARZE}

Es wurden zwei Harze der Firma Hans Schmid KG verwendet. Die Harze unterscheiden sich hinsichtlich der Aushärtungsgeschwindigkeit. Das langsam aushärtende ist ein Standardharz für Imprägnierung, das schnell härtende ist ein Gegenzugharz. Es handelt sich um reine Melaminharze ohne Harnstoff.

\subsubsection{TRÄGERPLATTEN}

Zwei $16 \mathrm{~mm}$ Spanplatten unterschiedlicher Hersteller wurden verwendet. Die Platten wurden aus jeweils einer Charge produziert. Beide Spanplatten werden von den Herstellern als Spanplatten für die Direktbeschichtung geführt. Die Spanplatte, die bei den meisten Versuchen verwendet wurde, hat laut den Industriepartnern eine gängige Oberflächenqualität. Die andere Spanplatte hat eine feinere Deckschicht und keinen Recyclinganteil.

Die restlichen Holzwerkstoffe wurden nur im Rahmen einer Versuchsreihe beschichtet und geprüft. Bei diesen Trägerplatten handelt es sich um handelsübliche Spanplatten, MDF, Sperrhölzer sowie Gipsund Brandschutzplatten unbekannter Hersteller. 
Tabelle 2: Parameter der verwendeten Trägerplatten.

\begin{tabular}{|c|c|c|}
\hline Holzwerkstoff & $\begin{array}{l}\text { Stärke } \\
{[\mathrm{mm}]}\end{array}$ & Anmerkung \\
\hline Spanplatte & 9 & \\
\hline Spanplatte & 11 & \\
\hline Spanplatte & 16 & "Standardplatte" (verwendet bei den meisten Versuchen) \\
\hline Spanplatte & 16 & feine Deckschicht, ohne Recyclinganteil \\
\hline Spanplatte & 25 & \\
\hline Grobspanplatte & 25 & grobe spanplatte, kein OSB \\
\hline $\mathrm{MDF}$ & 3 & \\
\hline $\mathrm{MDF}$ & 9 & \\
\hline $\mathrm{MDF}$ & 11 & \\
\hline Sperrholz & 8 & Birke, 5 Lagen \\
\hline Brandschutzplatte & 20 & \\
\hline Gipsplatte & 10 & \\
\hline
\end{tabular}




\subsection{Produktion VON Untersuchungsmaterial}

\subsubsection{IMPRÄGNIERUNG}

Es wurden vier Imprägnate produziert. Die Papiere wurden von Hans Schmid KG einstufig an einer industriellen Imprägnierstraße mit $105 \mathrm{~g} / \mathrm{m}^{2}$ beaufschlagt. Als Endfeuchte (Imprägnatfeuchte nach der Trocknung) wurde 6,2\% eingestellt. Es wurden zwei Formate hergestellt; $3000 \times 1250 \mathrm{~mm}^{2}$ für die industrielle Verpressung und $1500 \times 1000 \mathrm{~mm}^{2}$ (Abbildung 6) für Verpressungen im Technikum.

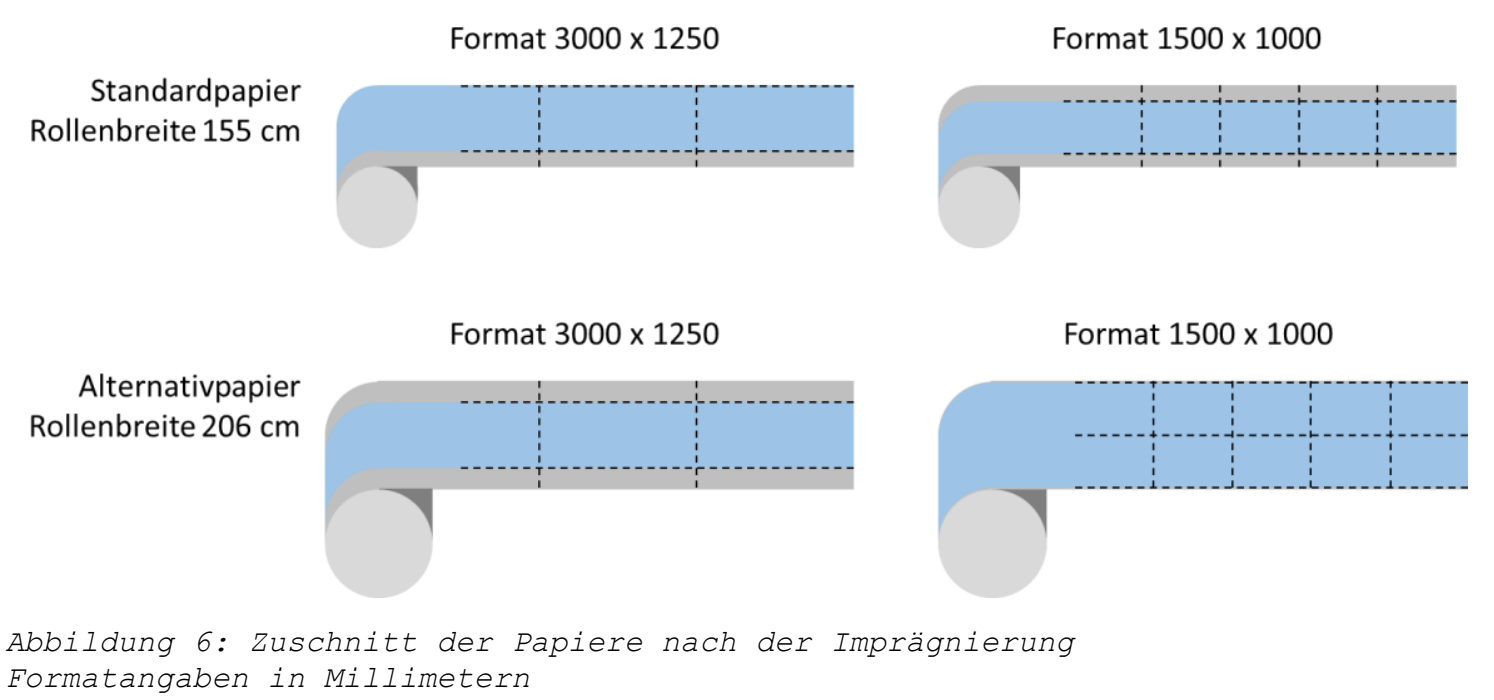

\subsubsection{BeSCHICHTUNGSVERFAHREN UND AUSHÄRTUNG FREIER FILME}

Zur Entwicklung und Optimierung von Prüfmethoden wurden wenige Plattenvarianten benötigt - diese allerdings in großem Umfang und möglichst homogen. Solche Platten wurden industriell verpresst. Für die Untersuchungen von Einflussgrößen auf die Rissbildung wurden viele Plattenvarianten in geringem Umfang benötigt. Dazu wurden Platten im Technikum des Fraunhofer WKI beschichtet. Es wurde sowohl mit dem gängigen Pressverfahren des Technikums als auch mit einem modifizierten, industrienahen Verfahren direktbeschichtet.

Unabhängig vom Pressverfahren wurden, sofern nicht anders angegeben, folgende Einstellungen gewählt:

- Pressdruck: $2,5 \mathrm{~N} / \mathrm{mm}^{2}$

- Presstemperatur: $185^{\circ} \mathrm{C}$

- Pressdauer: schnell härtendes Harz $35 \mathrm{~s}$, langsam härtendes Harz $45 \mathrm{~s}$

Untersuchungsmaterial wurde mit einem Mindestabstand von $10 \mathrm{~cm}$ vom Plattenrand entnommen. 


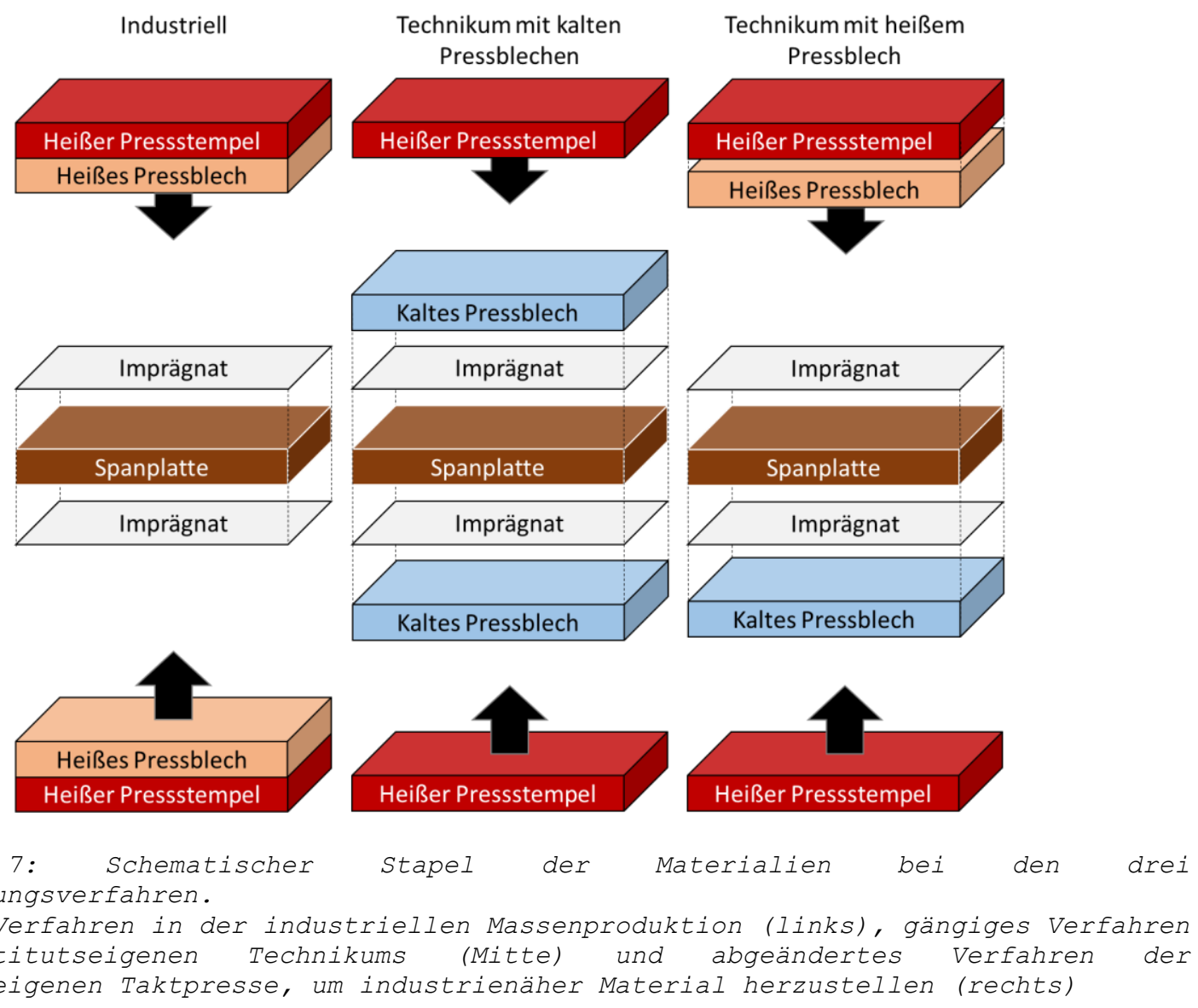

Abbildung 7 : Beschichtungsverfahren.

Gängiges Verfahren in der industriellen Massenproduktion (links), gängiges Verfahren des institutseigenen Technikums (Mitte) und abgeändertes Verfahren der institutseigenen Taktpresse, um industrienäher Material herzustellen (rechts)

\section{INDUSTRIELLE VERPRESSUNG}

Die industrielle Verpressung wurde bei Saréco Deutschland $\mathrm{GmbH}$ an einer industrietypischen Taktpresse durchgeführt. Auf einen Schlitten wird ein Imprägnat ausgebreitet. Eine Spanplatte wird automatisiert auf das Imprägnat abgelegt und von einem weiteren Imprägnat händisch bedeckt. Der Schlitten fährt in die Presse, legt den Stapel auf einem heißen Pressblech ab und fährt wieder raus. Die Presse fährt von oben einen heißen Pressstempel auf den Stapel und presst ihn zusammen. Das Imprägnat wird mit der Spanplatte quasiverschweißt. Nach dem Öffnen der Presse wird die Platte automatisiert mit Saugnäpfen aus der Presse gehoben und in einem Stapel abgelegt.

Industrielle Beschichtungen fanden im Format $3 \mathrm{~m}$ x 1,25 m statt.

\section{TECHNIKUMSVERPRESSUNG MIT KALTEN PRESSBLECH}

Bei der industriellen Verpressung sind die Pressbleche an den Pressstempeln befestigt und werden kontinuierlich beheizt. Bei der Technikumsverpressung werden die Pressbleche vor der Presse zur Bildung des Materialstapels verwendet und sind daher nicht vorgeheizt.

Vor der Taktpresse (Dieffenbacher TP 120/160/80) wird ein Stapel aus Imprägnaten, Spanplatte und kalten Pressblechen geschichtet (Abbildung 7). Der Stapel wird von einem Schlitten in die Presse auf einen heißen Pressstempel geschoben. Ein weiterer heißer Pressstempel fährt von oben auf den Stapel und presst inn zusammen. Die Pressstempel erhitzen die kalten Pressbleche, welche wiederum die Wärme an die Imprägnate und die Spanplatte weitergeben. Mit Ablauf der Pressdauer öffnet sich die Presse und die beschichtete Platte mit den nun heißen Pressbleche wird vom Schlitten aus der Presse gezogen. Das obere Pressblech wird abgehoben, die direktbeschichtete Platte entnommen und zum Abkühlen separat gelagert. 
Alle Technikumsverpressungen wurden mit Platten im Format 1,5 $\mathrm{m} \times 1,25 \mathrm{~m}$ und Imprägnaten im Format $1,5 \mathrm{~m} \times 1 \mathrm{~m}$ verpresst.

\section{$\underline{\text { TECHNIKUMSVERPRESSUNG MIT VORGEHEIZTEM PRESSBLECH }}$}

Um die Pressblechnutzung bei der Technikumsverpressung industrienäher zu gestalten, wurde das obere Pressbleche an der Presse befestigt. Dieses Pressblech wird bei einer Verpressung einer Spanplatte vorgeheizt. Erst danach wird der Stapel in die Presse geschoben und mit dem nun vorgeheizten Pressblech verpresst (Abbildung 7). Bei so beschichteten Platten wurde nur die Seite, die mit dem vorgeheizten Pressblech in Kontakt kam, geprüft.

Dieses Verfahren wurde auch genutzt um freie Filme zu produzieren. Bei freien Filmen handelt es sich um ausgehärtete Direktbeschichtungen ohne Trägerplatten. Zur Produktion wird lediglich ein ReleasePapier zwischen Imprägnat und Spanplatte gelegt werden. Release-Papiere werden bei der industriellen Produktion von CPL verwendet, um ein Verkleben des Imprägnats mit den Pressbändern zu verhindern. Bei der Produktion freier Filme verhindert das Release-Papier, dass das Imprägnat sich beim Aushärten mit der Spanplatte verbindet. Da bei diesem Pressverfahren lediglich die mit heißem Pressblech ausgehärtete Direktbeschichtung geprüft werden sollte, wurde auf das untere Imprägnat verzichtet. 


\subsection{EINZELNE METHOdEN}

\subsubsection{RISSANFÄLLIGKEIT}

Mit dieser Methode wird die Anfälligkeit einer direktbeschichteten Platte gegen Rissbildung quantifiziert. Die Methode ist eine Abwandlung der Risswiderstandsprüfung nach DIN EN 14323 (2014).

Aus der zu untersuchenden Platte werden Proben entnommen, deren Ränder gefast und Nut sowie Bohrloch in die Prüffläche eingebracht (Abbildung 8). Nach einer Akklimatisierung im Normklima, werden die Proben im Ofen belastet und anschließend die Länge entstandener Risse vermessen (Tabelle 3). Als Risslänge wird die direkte Entfernung beider Rissenden gemessen. Von jeder Probe wird eine kumulierte Risslänge berechnet. Der Mittelwert der kumulierten Risslängen aller Proben wird als Maß für die Rissanfälligkeit der Platte angegeben.

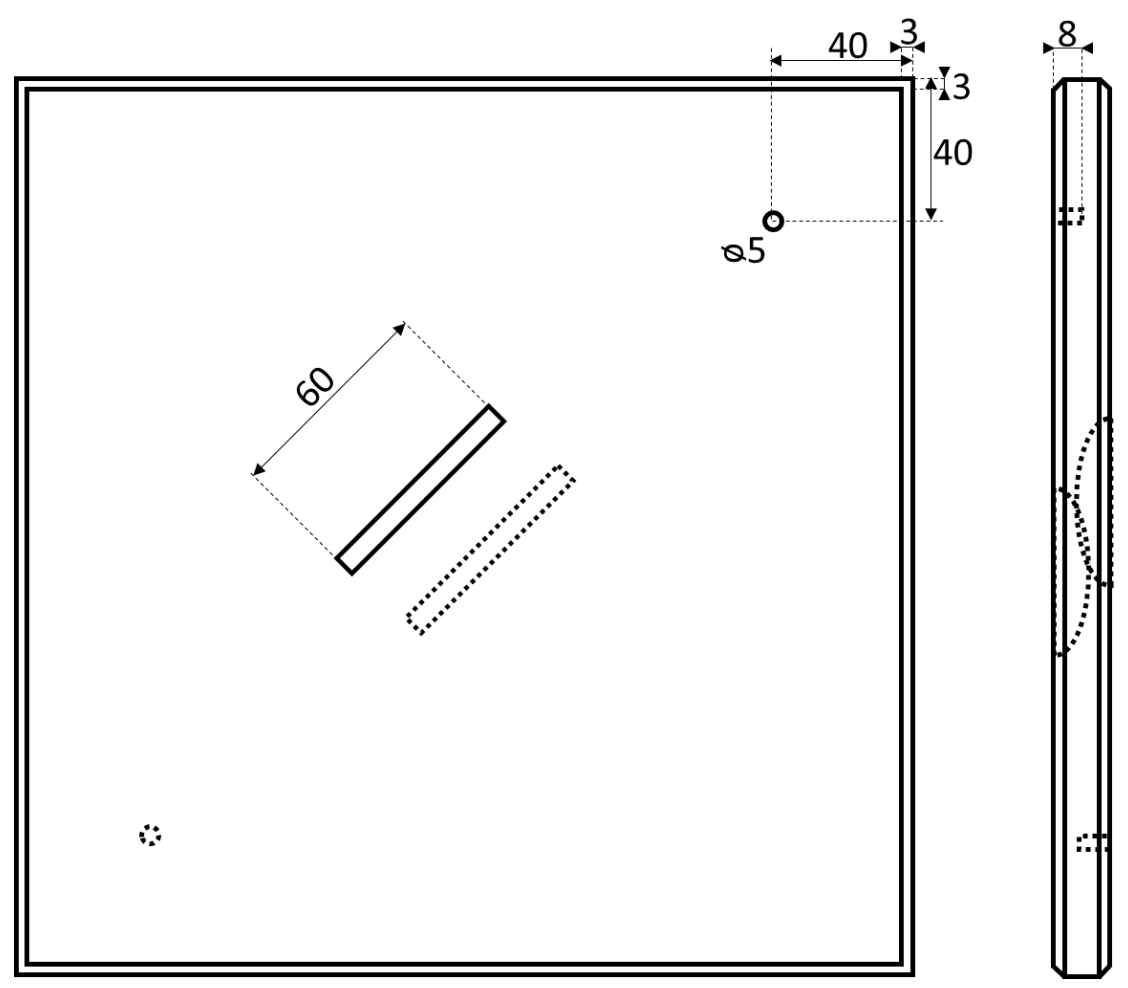

Abbildung 8: Auf- (links) und Seitenansicht (rechts) für eine Probe zur Messung der Rissanfälligkeit von Direktbeschichtungen

Tabelle 3: Parameter zur Rissanfälligkeitsmethode

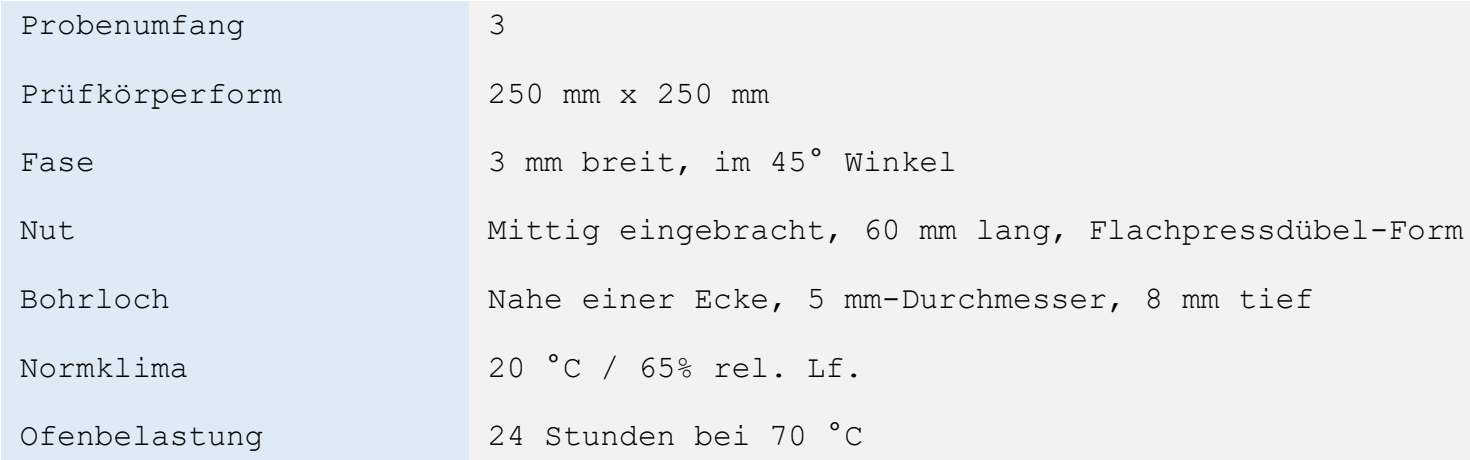



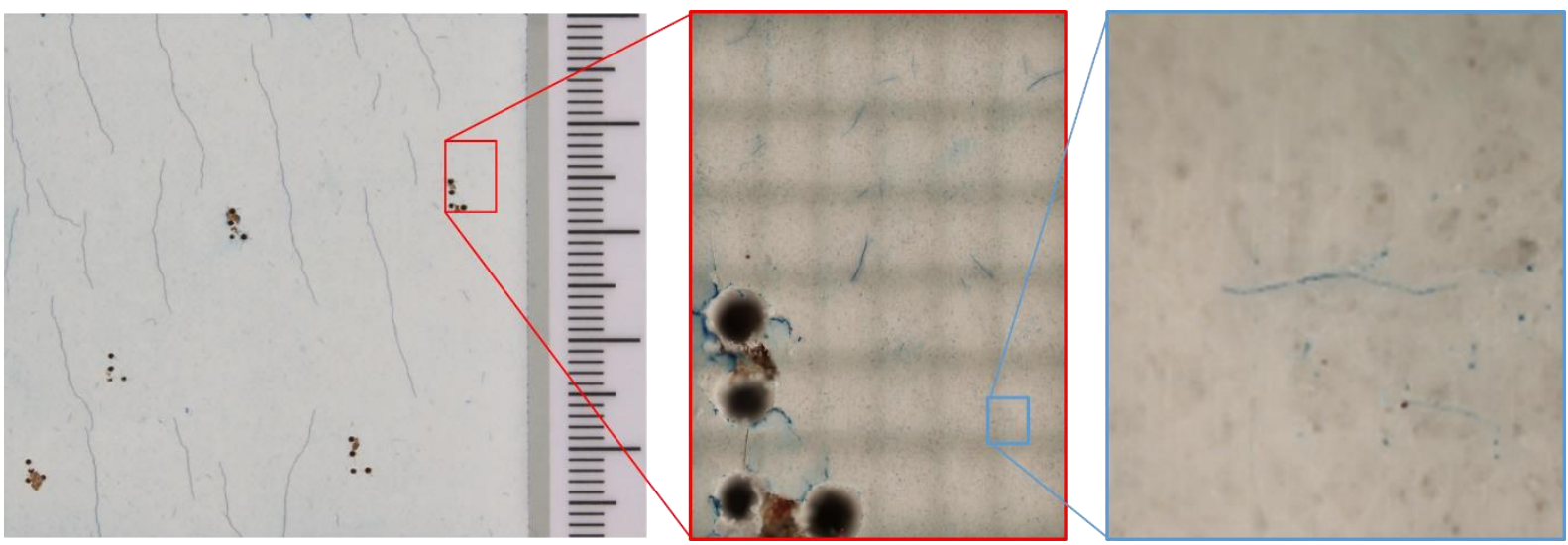

Abbildung 9: Verschiedene Vergrößerungen einer Platte.

Links ein Foto einer $5 \mathrm{~cm} \times 5 \mathrm{~cm}$ Probe, Mitte eine Aufnahme bestehend aus 36 mikroskopischen Einzelaufnahmen, rechts Vergrößerung eines Mikrorisses.

Neben den mit bloßem Auge sichtbaren Makrorissen treten in der Direktbeschichtung auch nur bei hoher Vergrößerung sichtbare Mikrorisse auf. Mit dieser Methode werden solche Mikrorisse quantifiziert.

Die zu untersuchende Oberfläche einer Probe wird mit Tuschierpaste eingerieben (Tabelle 4). Anschließend wird die Tuschierpaste mit Ethanol entfernt. Nur Risse sind auch nach der Reinigung mit Ethanol weiterhin eingefärbt. An drei Stellen wird unter einem Mikroskop entlang der gesamten Breite der Probe nach Mikrorissen gesucht. Bei dem verwendeten Mikroskop und einer Probenbreite von ca. $50 \mathrm{~mm}$ ergab sich dadurch eine Fläche von fast $150 \mathrm{~mm}^{2}$. Werden keine Mikrorisse gefunden, wird die Untersuchung beendet und der Probe eine Mikrorissdichte von $0 \mu \mathrm{m} / \mathrm{cm}^{2}$ zugewiesen. Wird mindestens ein Mikroriss gefunden, werden an 5 zufällig gewählten Stellen jeweils 36 mikroskopische Aufnahmen angefertigt und zu einer Großaufnahme (Stitching oder Multiple Image Alignment genannt) zusammengefügt. Die Längen aller Mikrorisse werden vermessen und mittels Formel 1 wird die Mikrorissdichte der Stelle berechnet. Als Mikrorissdichte der Platte wird das arithmetische Mittel der einzelnen Stellen gebildet.

Formel 1: Mikrorissdichte

$R_{\mu}=\frac{\sum l}{A}$

$\begin{array}{lll}R_{\mu} & {\left[\frac{\mu m}{\mathrm{~cm}^{2}}\right]} & \text { Mikrorissdichte } \\ l & {[\mu \mathrm{m}]} & \text { Einzelrisslänge } \\ A & {\left[\mathrm{~cm}^{2}\right]} & \begin{array}{l}\text { Abmessungen der } \\ \text { untersuchten } \\ \end{array} \\ & & \text { Stelle }\end{array}$

Tabelle 4: Parameter zur Quantifizierung von Mikrorissen

$\begin{array}{ll}\text { Untersuchte Stellen } & 5 \\ \text { Tuschierpaste } & \text { Diamant Tuschierpaste für Oberflächenprüfungen - blau } \\ \text { Abmessungen der Großaufnahme } & 6540 \mu \mathrm{m} \times 4665 \mu \mathrm{m} \\ \text { Mikroskop } & \text { Zeiss Axioplan (200-fache Vergrößerung) } \\ \text { Software } & \text { NIS Elements } 5.11\end{array}$




\subsubsection{PROJEKTION VON MAKRORISSEN}

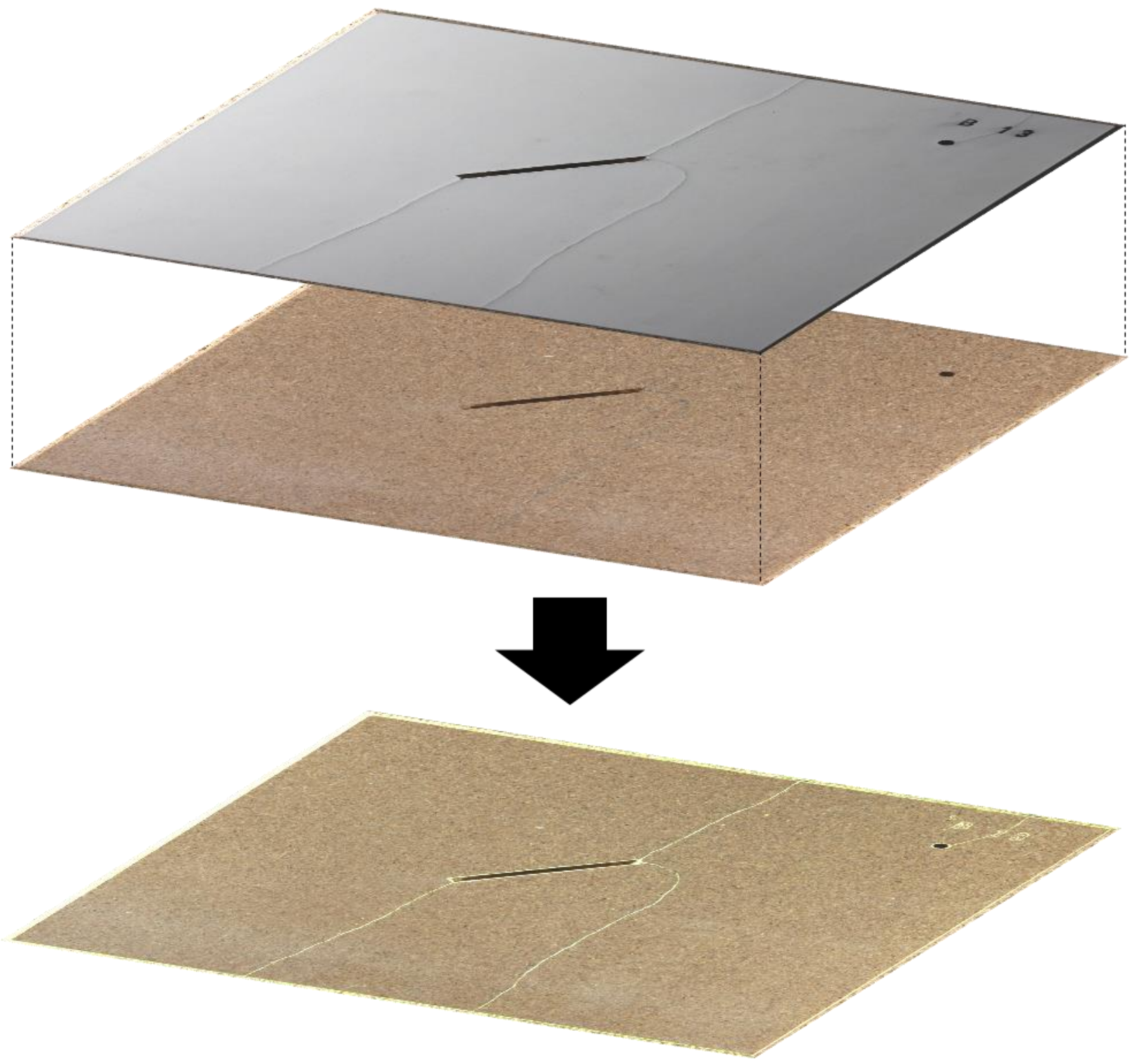

Abbildung 10: Arbeitsschritte bei der Rissprojektion.

Mit der Rissprojektion lässt sich ein Einfluss des Untergrunds auf die Risslage und den Rissverlauf prüfen.

Von einer auf Rissanfälligkeit geprüften Probe wird die Oberfläche fotografiert, die Direktbeschichtung heruntergeschliffen und die Oberfläche erneut fotografiert. Mit einem Bildbearbeitungsprogramm werden die beiden Fotos übereinander ausgerichtet (Abbildung 10) und das Foto mit den Rissen wird mit einem vorinstallierten Filter des Bildbearbeitungsprogramms skeletiert (Tabelle 5). Dadurch werden die Risse der Direktbeschichtung auf dem geschliffenen Untergrund angezeigt.

Tabelle 5: Parameter zur Projektion von Makrorissen

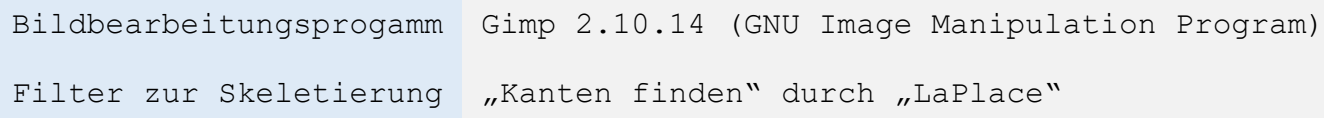




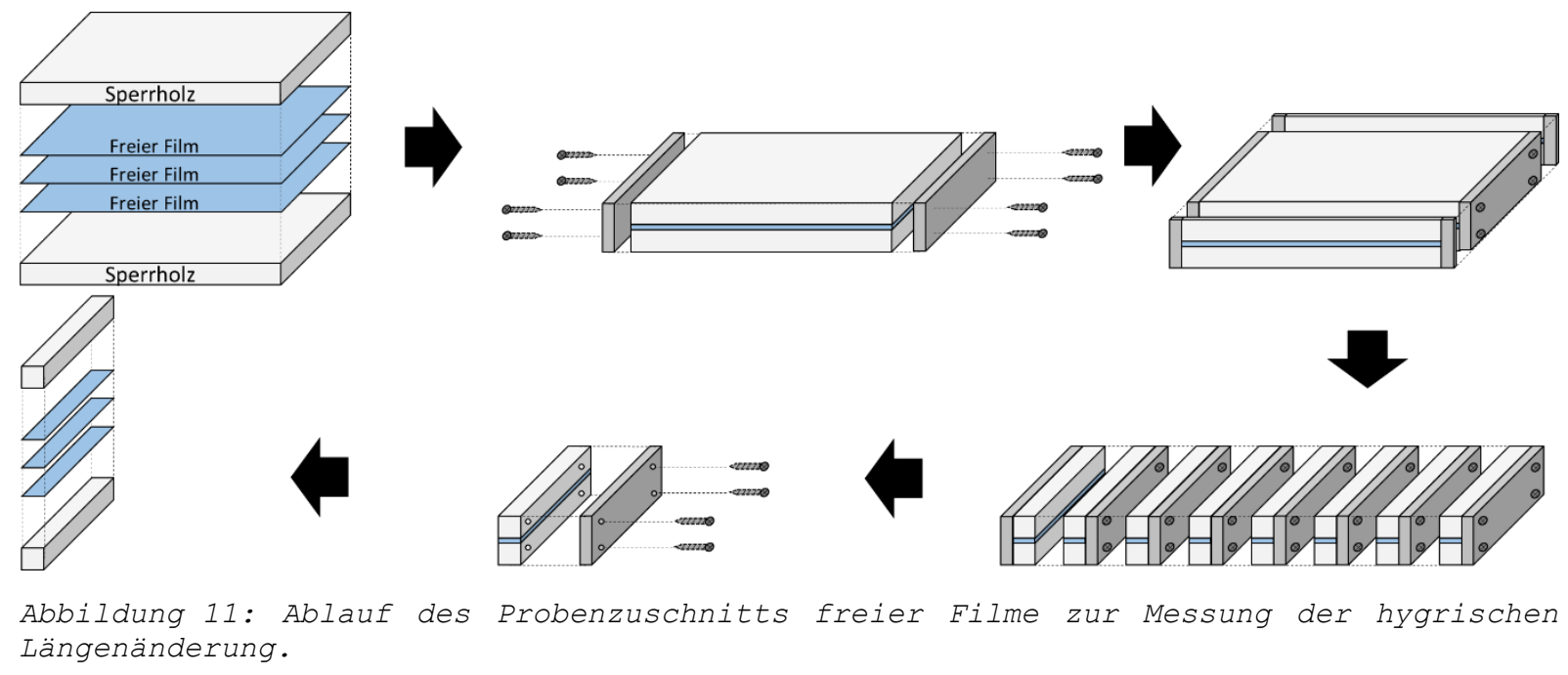

Freie Filme sind äußerst zerbrechlich. Es müssen daher bei der Probenpräparation Vorkehrungen getroffen werden. Dazu werden die freien Filme zwischen Sperrhölzern eingespannt und Abschnitte in der gewünschten Probenform abgesägt (Abbildung 11). Einseitig befestigte Bretter dienen dabei zur mittelbaren Fixierung der Stapel. Zur besseren Handhabung werden die Proben auf Blättern kleinflächig mit Klebeband fixiert. Jeweils ein Blatt mit Proben eines freien Films durchläuft Klimata mit steigender bzw. fallender relativer Luftfeuchte (Tabelle 8, in Anlehnung an DIN EN 318 (2002)).

Die hygrischen Längenänderungen der freien Filme lassen sich nicht wie die von Holzwerkstoffen messen. Der mechanische Längenmesser würde die Proben verformen oder sogar zerbrechen. Daher werden die Prüfkörper stattdessen plangedrückt und gescannt. Die Bilder werden kalibriert und beide Längskanten der Prüfkörper vermessen und gemittelt. Quellmaße werden nach Formel 2 berechnet. Die gravimetrischen Messungen wurden in einem separaten Versuch (Kapitel 3.3.7) durchgeführt.

Tabelle 8: Parameter zur Messung hygrischer Längenänderung freier Filme

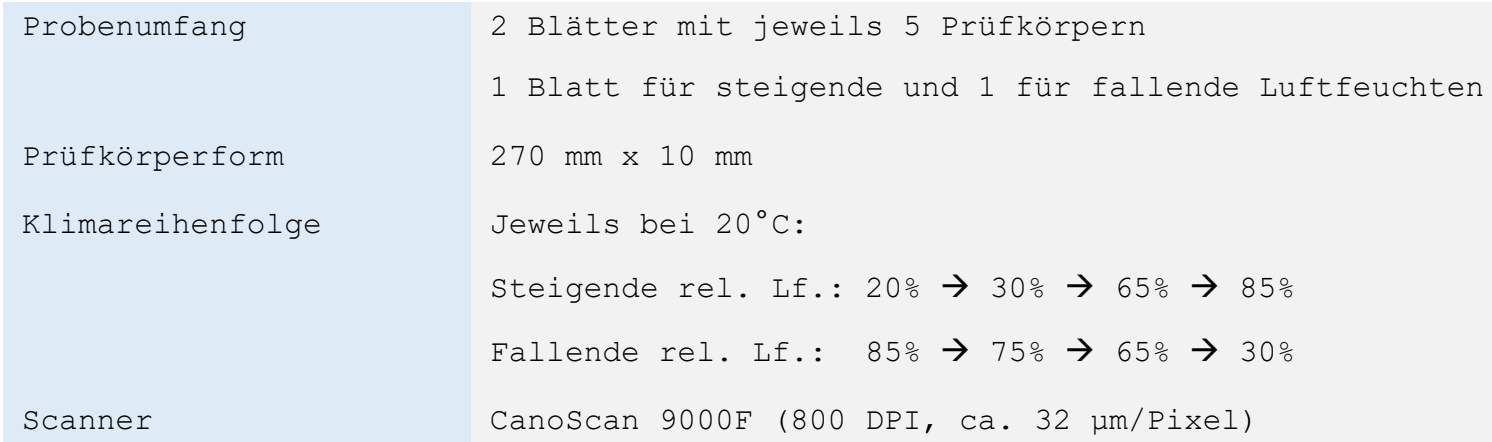




\subsubsection{SORPTIONSVERHALTEN FREIER FILME}

Bei der hygrischen Längenänderung von Holzwerkstoffen nach DIN EN 318 (2002) werden auch die Massen mitaufgezeichnet. Bei freien Filmen wurde die gravimetrische Messung in dieses separate Verfahren ausgelagert. Aufgenommen werden die Daten mittels DVS (Dynamic Vapour Sorption).

DVS besteht aus einer Klimakammer mit feinauflösender Waage. Zu Pulver zermahlene freie Filme werden auf die Waage gelegt und durchlaufen einen Klimazyklus. Klima und Probenmasse werden kontinuierlich aufgenommen. Nach Erreichen der Massekonstanz (Gleichgewichtsfeuchte) in einem Klima wird das nächste Klima angefahren (Tabelle 9).

Tabelle 9: Parameter zur Messung des Sorptionsverhaltens freier Filme

\begin{tabular}{|c|c|}
\hline Probenumfang & 1 \\
\hline Prüfkörpermasse & $\mathrm{Ca} \cdot 20 \mathrm{mg}$ \\
\hline Klimareihenfolge & $\begin{array}{l}\text { Bei } 20^{\circ} \mathrm{C} \text { und in 5\% rel. Lf. Schritten: } \\
0 \% \text { rel. Lf. }->100 \% \text { rel. Lf. }->0 \% \text { rel. Lf. }\end{array}$ \\
\hline $\begin{array}{l}\text { Kriterium für } \\
\text { Massekonstanz }\end{array}$ & $\begin{array}{l}\text { Durchschnittliche Massenzunahme pro Minute für } 10 \text { Minuten } \\
\text { unter 0,002\% (analog HimmeL; MAI 2015) }\end{array}$ \\
\hline Messgerät & $\begin{array}{l}\text { DVS: DVS Advantage (Surface Measurement Systems Ltd.), } \\
\text { Genauigkeit Gravimetrie: 0,1 } \mu \mathrm{g}, \\
\text { Genauigkeit Luftfeuchte: 0,5\% rel. Lf. } \\
\text { Mühle: Retsch ZM } 200 \text { (0,12 mm Maschen) }\end{array}$ \\
\hline
\end{tabular}

\subsubsection{DiFFUSIONSWIDERSTAND FREIER FILME}

Der Diffusionswiderstand wird mittels Dry-Cup und Wet-Cup bestimmt (DIN EN ISO 12572:2017). Bei diesen Methoden wird gemessen, wie schnell ein Feuchteaustausch zwischen humiden und ariden Klimata stattfindet, wenn diese durch das Untersuchungsmaterial getrennt sind.

In Gefäße wird Trockengel (bei Dry-Cup) oder Natriumcarbonat (bei Wet-Cup) gegeben. Ein Abschnitt des freien Films wird als Membran über die Öffnung des Gefäßes gespannt. Die Masse der Gefäße werden während der Lagerung bei $23^{\circ} \mathrm{C} / 50 \%$ rel. Lf. regelmäßig bestimmt (Tabelle 10). Die Wasserdampfdiffusionsäquivalente Luftschichtdicke bzw. die Wasserdampfdiffusionswiderstandszahl berechnet sich aus der Probenmasse nach Formel 3 und Formel 4.

Tabelle 10: Parameter zur Messung des Diffusionswiderstands freier Filme

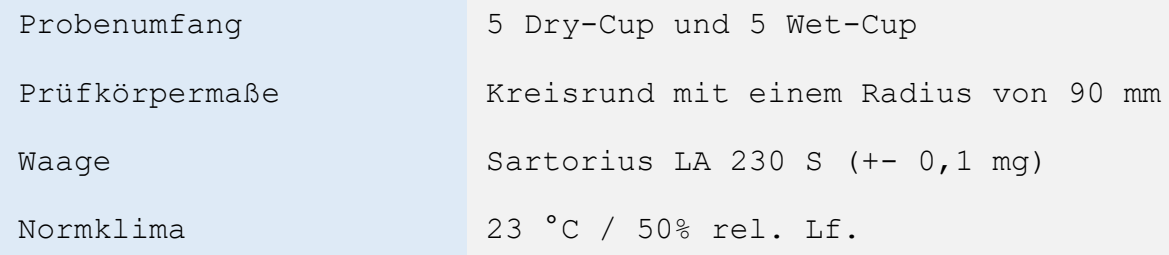


Formel 3: Wasserdampfdiffusionswiderstandszahl nach DIN EN ISO 12572 (2017)

$\mu=\frac{\frac{0,086 * p_{0}}{k_{d} * T * p} *\left(\frac{T}{273}\right)^{1,81} * A * \frac{\Delta p}{G}}{d}$

Forme1 4: Wasserdampfdiffusionsstrom

$G=\frac{\sum_{i} \frac{m_{2}-m_{1}}{t_{2}-t_{1}}}{i}$

\begin{tabular}{|c|c|c|}
\hline$G$ & {$[\mathrm{~kg} / \mathrm{s}]$} & $\begin{array}{l}\text { Wasserdampfdiffusions- } \\
\text { strom }\end{array}$ \\
\hline$m$ & {$[k g]$} & Masse des Prüfkörpers \\
\hline$t$ & {$[s]$} & $\begin{array}{l}\text { Zeitpunkt der } \\
\text { Massemessung }\end{array}$ \\
\hline$p_{0}$ & {$[h P a]$} & $\begin{array}{l}\text { Normaler Luftdruck } \\
(1013,25 \mathrm{hPa})\end{array}$ \\
\hline$k_{d}$ & {$\left[\frac{\mathrm{J}}{\mathrm{kgK}}\right]$} & $\begin{array}{l}\text { Gaskonstante von } \\
\text { Wasserdampf }\end{array}$ \\
\hline$T$ & {$[K]$} & Temperatur \\
\hline$p$ & {$[\mathrm{~Pa}]$} & Luftdruck \\
\hline$A$ & {$\left[m^{2}\right]$} & Probekörperfläche \\
\hline$\Delta p$ & {$[\mathrm{~Pa}]$} & $\begin{array}{l}\text { Differenz der } \\
\text { Wasserdampfteildrücke }\end{array}$ \\
\hline$d$ & {$[m]$} & $\begin{array}{l}\text { mittlere } \\
\text { Probekörperdicke }\end{array}$ \\
\hline
\end{tabular}

\subsubsection{DifFusIONSWIDERSTAND VON PLATTEN}

Bei der Bestimmung des Diffusionswiderstands freier Filme sind viele Prüfkörper gerissen. Der Diffusionswiderstand konnte an diesen Proben nicht bestimmt werden. Als alternative Methode wurden daher Messungen an direktbeschichteten Spanplatten durchgeführt. Gemessen wird, wieviel Feuchtigkeit durch die Direktbeschichtung verdunstet.

Die Ränder von im Normklima akklimatisierte Proben werden mit Silikon abgedichtet. Die Platten werden gewogen, bei $70^{\circ} \mathrm{C}$ temperiert und abschließend erneut gewogen (Tabelle 11). Die prozentuale Massenabnahme dient als Maß für den Diffusionswiderstand.

Tabelle 11: Parameter zur Messung des Diffusionswiderstands von Platten

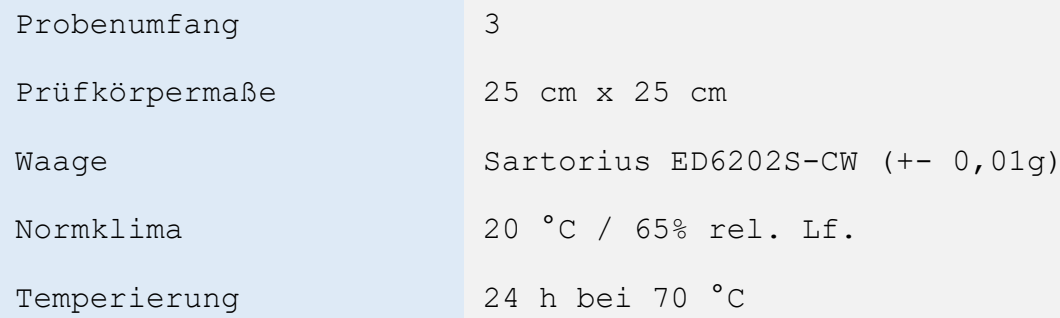




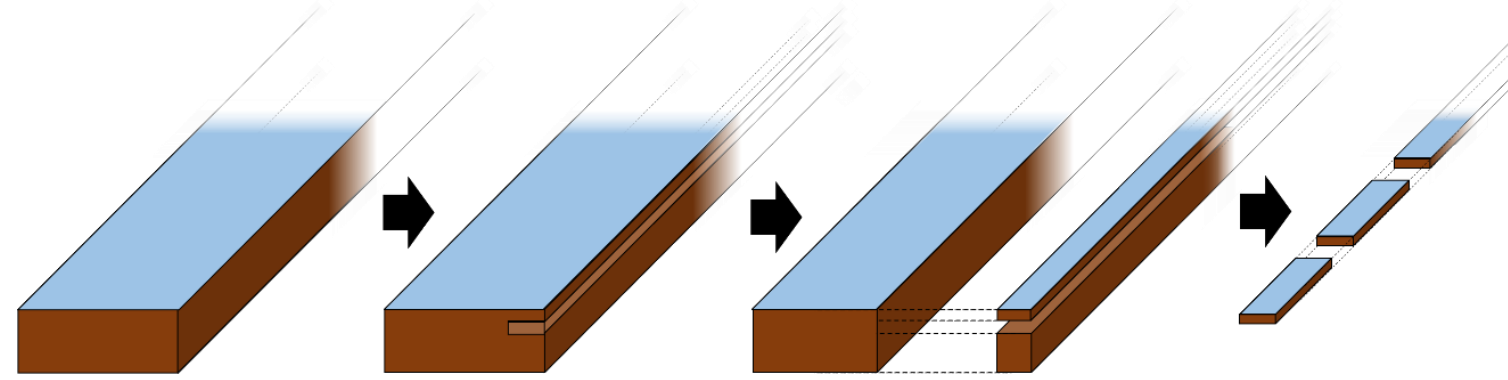

Abbildung 12: Produktionsschritte direktbeschichteter Platten zu Prüfkörpern für die Messung von Eigenspannungen

Spannungen zwischen der Direktbeschichtung und der Spanplatte können zu Verformungen führen. Dieser Effekt wurde genutzt, um die Eigenspannungen abzuschätzen. Das Stichmaß der Verformung dient als Maß für die Eigenspannungen.

Die Seite von direktbeschichteten Platten wird dicht unter der Oberfläche mit einer Fräse eingekerbt (Abbildung 12). An einer Kreissäge wird von der eingekerbten Seite ein Streifen runtergeschnitten. Der Streifen aus Spanplattendeckschicht und Direktbeschichtung wird abgelängt. Die Proben werden erst in einem ariden, dann in einem humiden Klima bis zur Massekonstanz gelagert (Tabelle 12). In beiden Klimata werden die Stichmaße der Proben (Abbildung 13) mit einem Messkeil vermessen.

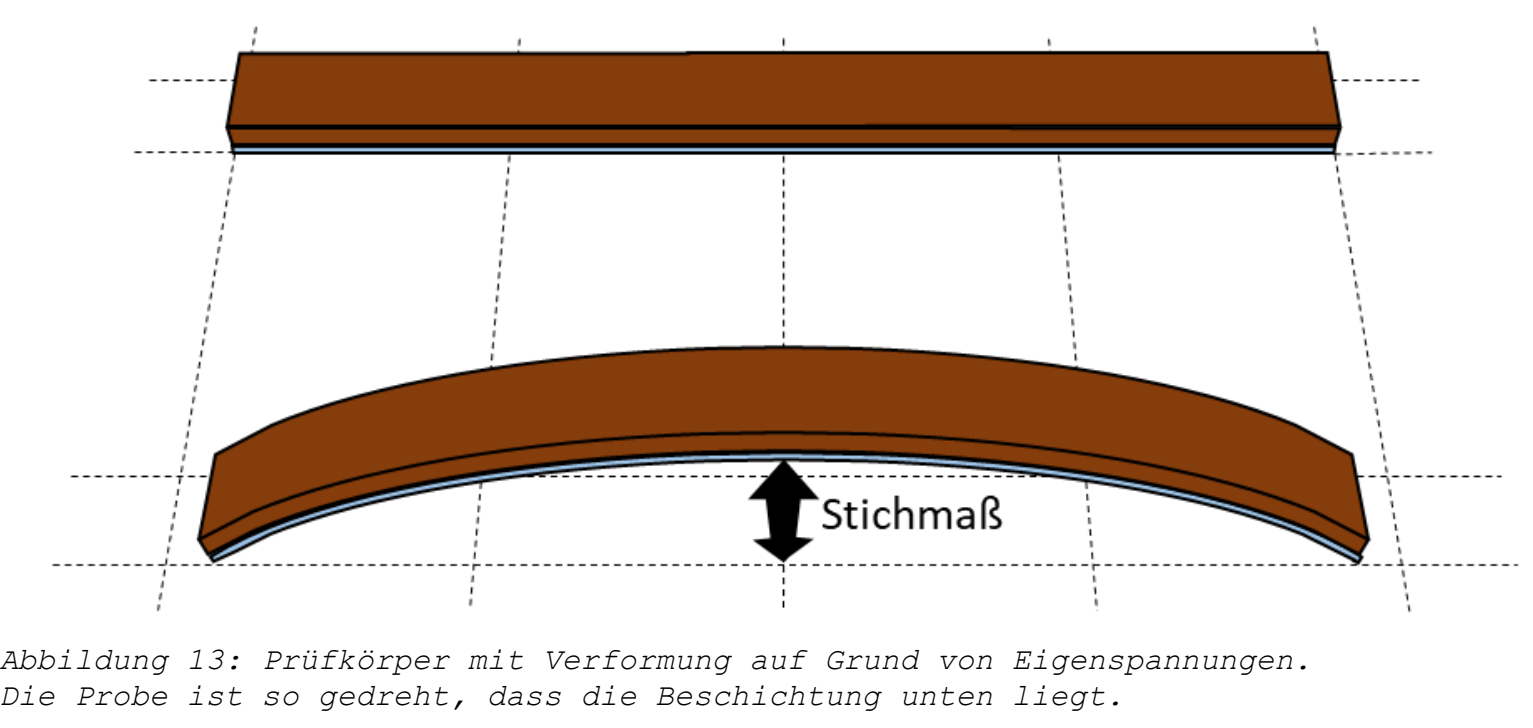

Tabelle 12: Parameter zur Messung von Eigenspannungen

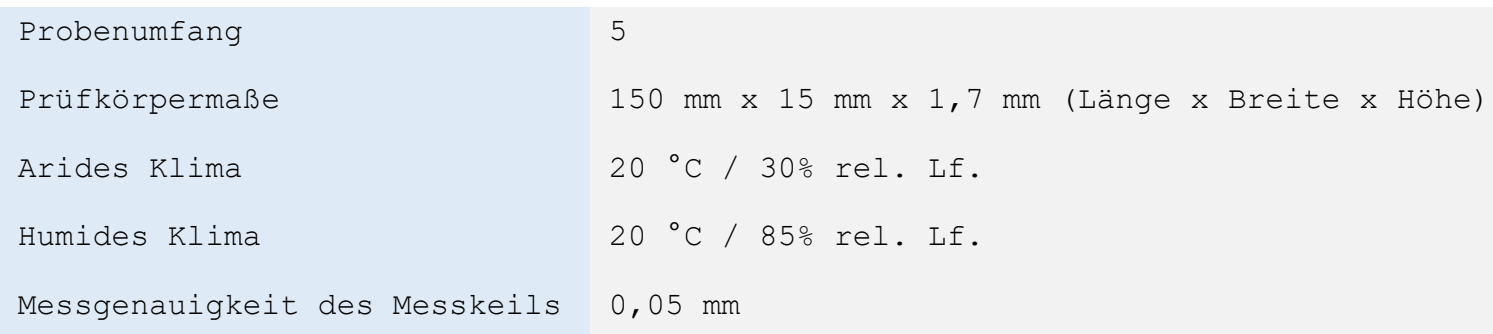




\subsubsection{BiEGEPRÜFUNG VON HOLZWERKSTOFFEN}

Bei der Biegeprüfung kommt es zu einer Spannungsverteilung im Prüfkörper; eine Prüfkörperseite wird auf Zug und die gegenüberliegende auf Druck belastet. Wird eine direktbeschichtete Spanplatte auf Biegung belastet, lässt sich in einer der Direktbeschichtungen eine rissverursachende Zugspannung nachstellen und messen.

Biegeprüfungen werden gemäß DIN EN 310 (1993) durchgeführt. Abweichend zur Norm wird lediglich senkrecht zur Produktionsrichtung geprüft (Tabelle 13). Die Prüfkörper werden so in die Maschine eingelegt, dass die mit heißem Pressblech beschichtete Direktbeschichtung auf Zug belastet wird.

Tabelle 13: Parameter zur Biegeprüfung von Holzwerkstoffen

$\begin{array}{ll}\text { Probenumfang } & 6 \\ \text { Prüfkörpermaße } & 370 \mathrm{~mm} \times 50 \mathrm{~mm} \\ \text { Normklima } & 20^{\circ} \mathrm{C} / 65 \% \mathrm{rel} \text { Lf. } \\ \text { Messschieber } & \text { Mitutoyo CD-15DC } \\ \text { Vorkraft } & 30 \mathrm{~N} \\ \text { Prüfgeschwindigkeit } & 6 \mathrm{~mm} / \mathrm{Min} \\ \text { Stützweite } & 320 \mathrm{~mm} \\ \text { Zugmaschine } & \text { Zwick Z1474 } 30 \mathrm{kN}\end{array}$

\subsubsection{Model ZUR BereChnUng MeChANISCHEN KenNWERTEN DER DiREKTBESCHICHTUNG}

Mechanische Kennwerte einer irreversibel aufgetragenen Beschichtung lassen sich näherungsweise über Biegeprüfungen bestimmen. Mit dem verwendeten mathematischen Model lassen sich Zugfestigkeit und Elastizitätsmodul der Direktbeschichtung ohne Einfluss der Trägerplatte berechnen.

An der direktbeschichteten Platte werden Biegeprüfungen (Kapitel 3.3.11) durchgeführt. Aus dem Protokoll der Biegeprüfung wird ein Messwert aus dem linearen Verformungsbereich gewählt. Ausgehend von der Einzellast $F$ und Verformung $d_{\text {mess }}$ des gewählten Messwerts wird mittels Zielwertsuche das Elastizitätsmodul der Direktbeschichtung bestimmt, bei dem die Verformungsdifferenz (Formel 5) 0 ergibt. Dafür wurde die Zielwertsuche von Microsoft Office Excel verwendet. Anschließend wird die Zugfestigkeit nach Formel 6 berechnet. 
Formel 5: Verformungsdifferenz

$$
d_{\text {Diff }}=\frac{F * l^{3}}{48 * E_{D} * I}-d_{m e s s}
$$

\begin{tabular}{|c|c|c|}
\hline$F$ & {$[N]$} & $\begin{array}{l}\text { Einzellast bei } \\
\text { der Verformung } \\
d_{\text {mess oder }} \\
\text { beim Bruch }\end{array}$ \\
\hline$l$ & {$[\mathrm{~mm}]$} & Auflagerabstand \\
\hline$E_{S}$ & {$\left[\mathrm{~N} / \mathrm{mm}^{2}\right]$} & $\begin{array}{l}\text { E-Modul der } \\
\text { Spanplatte }\end{array}$ \\
\hline$E_{D}$ & {$\left[\mathrm{~N} / \mathrm{mm}^{2}\right]$} & $\begin{array}{l}\text { E-Modul der } \\
\text { Direktbesch. }\end{array}$ \\
\hline$h_{S}$ & {$[\mathrm{~mm}]$} & $\begin{array}{l}\text { Höhe der } \\
\text { Spanplatte }\end{array}$ \\
\hline$h_{D}$ & {$[\mathrm{~mm}]$} & $\begin{array}{l}\text { Höhe der } \\
\text { Direktbesch. }\end{array}$ \\
\hline$b$ & {$[\mathrm{~mm}]$} & $\begin{array}{l}\text { Breite des } \\
\text { Prüfkörpers }\end{array}$ \\
\hline$d_{m e s s}$ & {$[\mathrm{~mm}]$} & $\begin{array}{l}\text { Gemessene } \\
\text { Verformung bei } \\
\text { der Einzellast } \\
F\end{array}$ \\
\hline
\end{tabular}

Formel 6: Festigkeit der Direktbeschichtung

$F_{\text {max }, \mathrm{D}}=M * \frac{h_{D}+h_{S}-a}{I}$

Formel 7: Bemessungsmoment

$M=\frac{F * l}{4}$

Formel 8: korrigierte Breite der Spanplatte

$b_{k}=\frac{E_{S}}{E_{D}} * b$

Formel 9: Flächenträgheitsmoment

$I=\left(b * \frac{h_{D}^{3}}{6}\right)+\left(b_{k} * \frac{h_{S}^{3}}{12}\right)$

Formel 10: Schwerpunkt

$a=\frac{\left(b * h_{D} *\left(h_{S}+\frac{3}{2} h_{D}\right)\right)+\left(b_{k} * h_{S} *\left(h_{D}+\frac{h_{S}}{2}\right)\right)+\left(b * \frac{3}{2} h_{D}\right)}{b * h_{D}+b_{k} * h_{S}+b * h_{D}}$ 
3.3.13 ZUGPRÜFUNG FREIER FILME UND IMPRÄGNIERPAPIERE
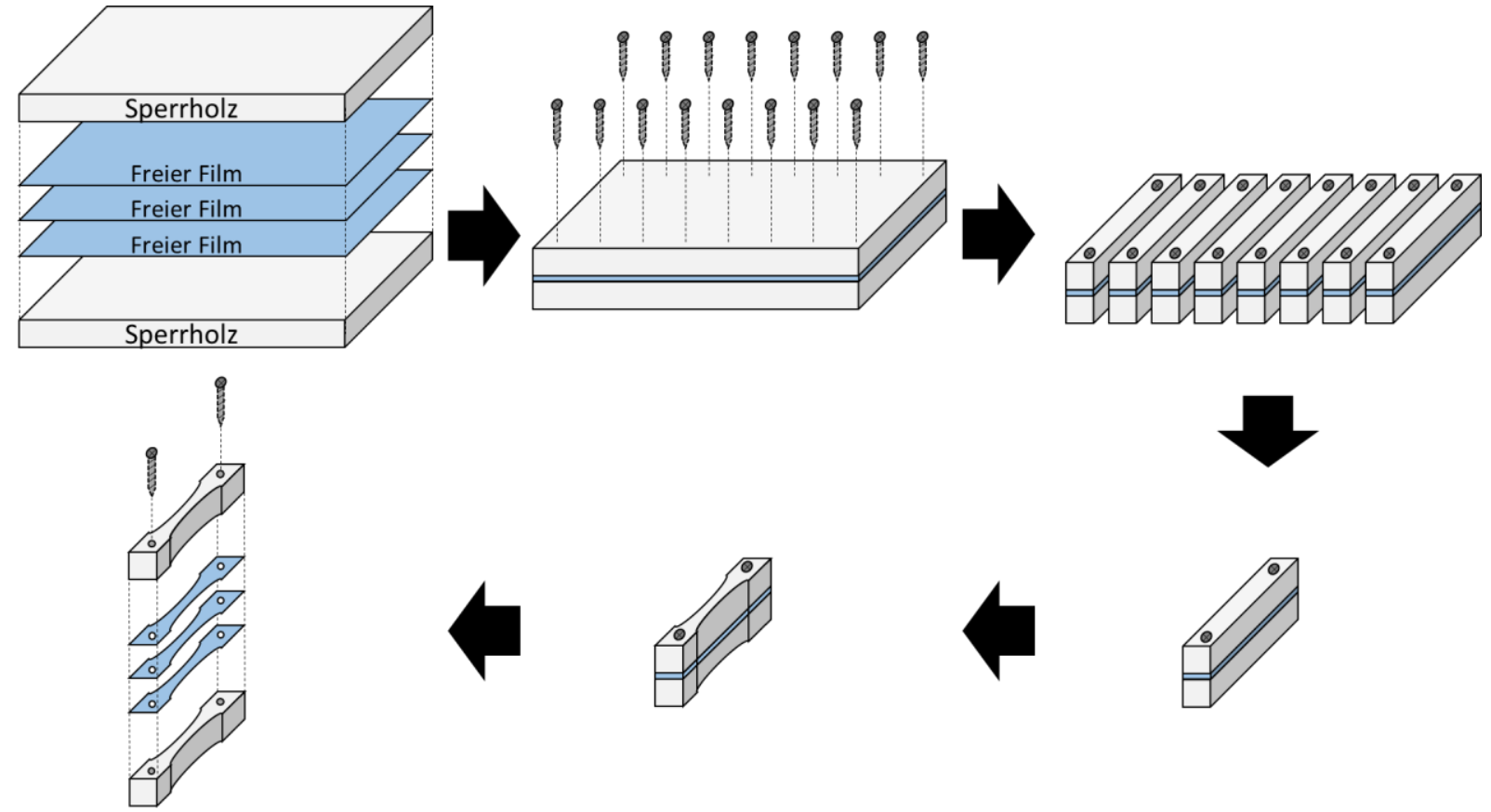

Abbildung 14: Produktionsschritte vom freien Film bzw. Papieren zu Prüfkörpern für Zugversuche.

Risse in Direktbeschichtungen entstehen durch Zugspannungen. Mit der Zugprüfung lässt sich eine solche Beanspruch nachstellen und wichtige mechanische Kennwerte einer Direktbeschichtung ermitteln.

Freie Filme sind äußerst zerbrechlich. Zur Probenpräparation müssen sie daher zwischen Sperrhölzern mit Schrauben stabilisiert werden (Abbildung 7). Mit einer Tischkreissäge werden Stäbe abgeschnitten und in jedem Stab wird eine Knochenform eingefräst. Die Proben erhalten ein Speckle-Muster (feine Tropfen aufgetragen mit einem Aerosolspray), werden bei Normklima akklimatisiert und einer Zugprüfung unterzogen (Tabelle 14). Kurz vor der Prüfung werden Dicke und Breite an der Verjüngung mit einem Messschieber aufgenommen.

Tabelle 14: Parameter zur Zugprüfung freier Filme und Imprägnierpapiere

\begin{tabular}{|c|c|}
\hline Probenumfang & 10 \\
\hline Prüfkörperform & $\begin{array}{l}\text { Knochenform Typ } 5 \text { (DIN EN ISO 527-3:2003) mit } 15 \text { mm Breite } \\
\text { an der Verjüngung }\end{array}$ \\
\hline Normklima & $20^{\circ} \mathrm{C} / 65 \%$ rel. Lf. \\
\hline Messschieber & Mitutoyo CD-15DC \\
\hline Vorkraft & $1 \mathrm{~N}$ \\
\hline Prüfgeschwindigkeit & $1 \mathrm{~mm} / \mathrm{min}$ \\
\hline Zugmaschine & 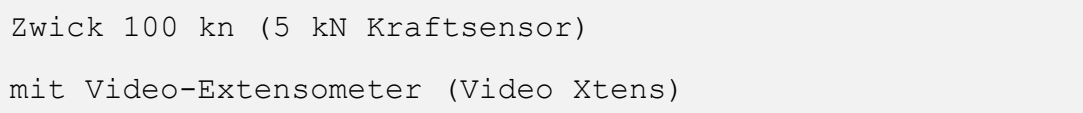 \\
\hline
\end{tabular}




\subsubsection{Zero-SPAN-PRÜFUng Von PAPIER}

Bei der Zugprüfung von Papier kommt es zu einem auseinanderziehen des Faservlieses. Um mechanische Kennwerte des Papiers ohne Auseinandergleiten der Fasern zu ermitteln, wurden ZeroSpan-Prüfungen durchgeführt. Zero-Span ist ein Zugversuch, bei dem die Probenklemmen direkt aneinander liegen. Die Spannweite (Span) zwischen den Klemmen ist 0 (Zero).

Das verwendete Gerät (Tabelle 15) gibt für den Bruch den aufgewendeten Druck an. Dieser wird mit der Formel 11 in die Festigkeit bzw. mit Formel 12 in die Reißlänge umgerechnet.

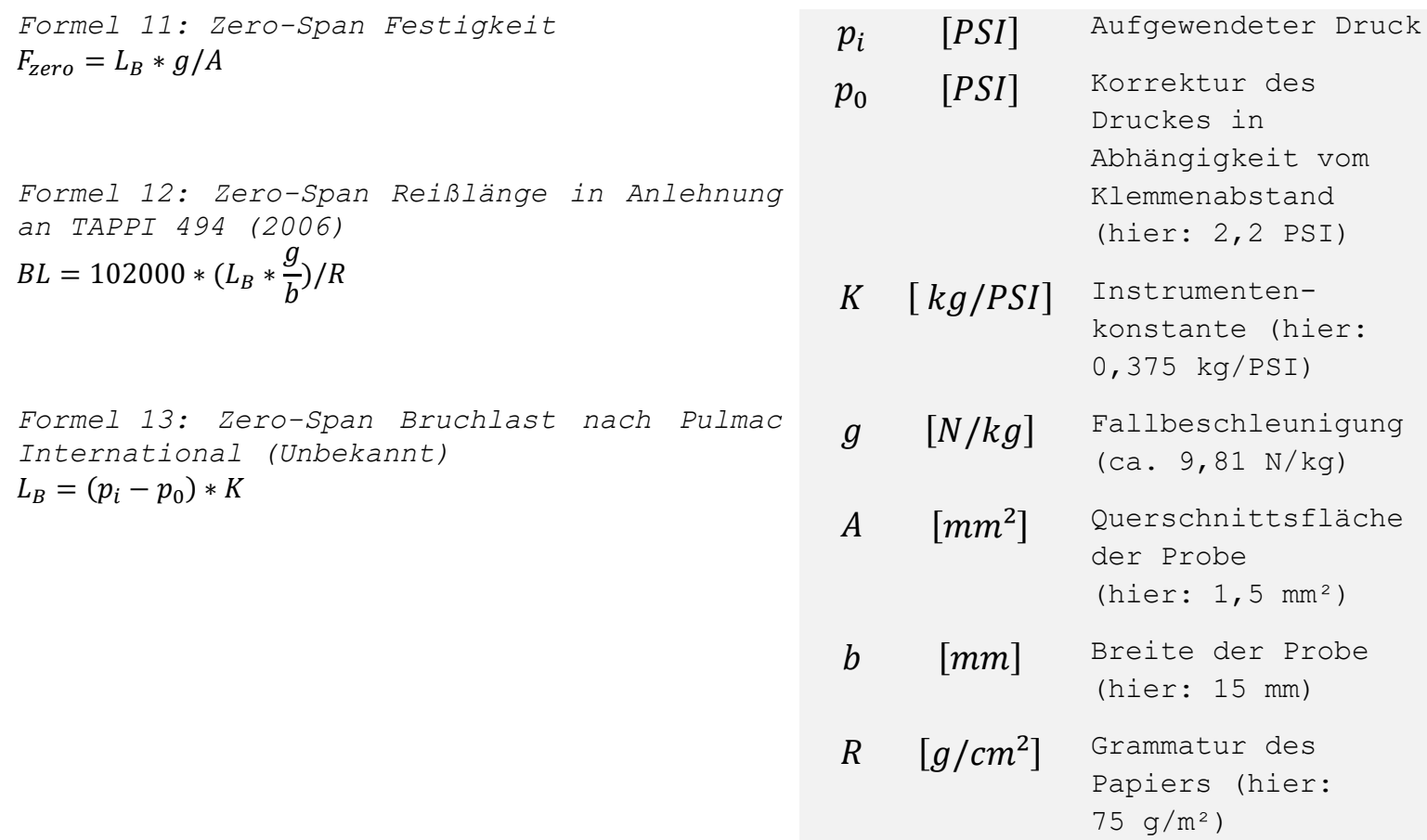

Tabelle 15: Parameter zur Zero-Span-Prüfung von Papier

$\begin{array}{ll}\text { Probenumfang } & 15 \\ \text { Prüfkörpermaße } & 70 \mathrm{~mm} \times 15 \mathrm{~mm} \\ \text { Messgerät } & \text { Pulmac Z-Span } 1000 \\ \text { Anpressdruck } & 60 \text { PSI }\end{array}$




\subsubsection{AUSHÄRTUNGSGRAD MITTELS PULVERHYDROLYSE}

Bei der Pulverhydrolyse macht man sich die Abhängigkeit der Säureresistenz vom Aushärtungsgrad bei Direktbeschichtungen zu nutze. Es wird gemessen, wieviel Melamin mit Säure aus einem Pulver der Direktbeschichtung gelöst werden kann.

Mit Schleifpapier wird ein Pulver von der Direktbeschichtung gewonnen. In Fläschchen portioniertes Pulver wird in einem Wasserbad temperiert. Bei Erreichen der Temperatur wird ebenfalls temperierte Salzsäure hinzugegeben. Die Salzsäure wird nach Ablauf einer definierten Zeit aufgenommen, filtriert und UV-spektrometrisch vermessen (Tabelle 16). Aus der Extinktion lässt sich die Masse hydrolysierten Melamins als Maß für den Aushärtungsgrad bestimmen. Für das genutzte Spektrometer lässt sich die Extinktion mittels Formel 14 bzw. Formel 15 umrechnen.

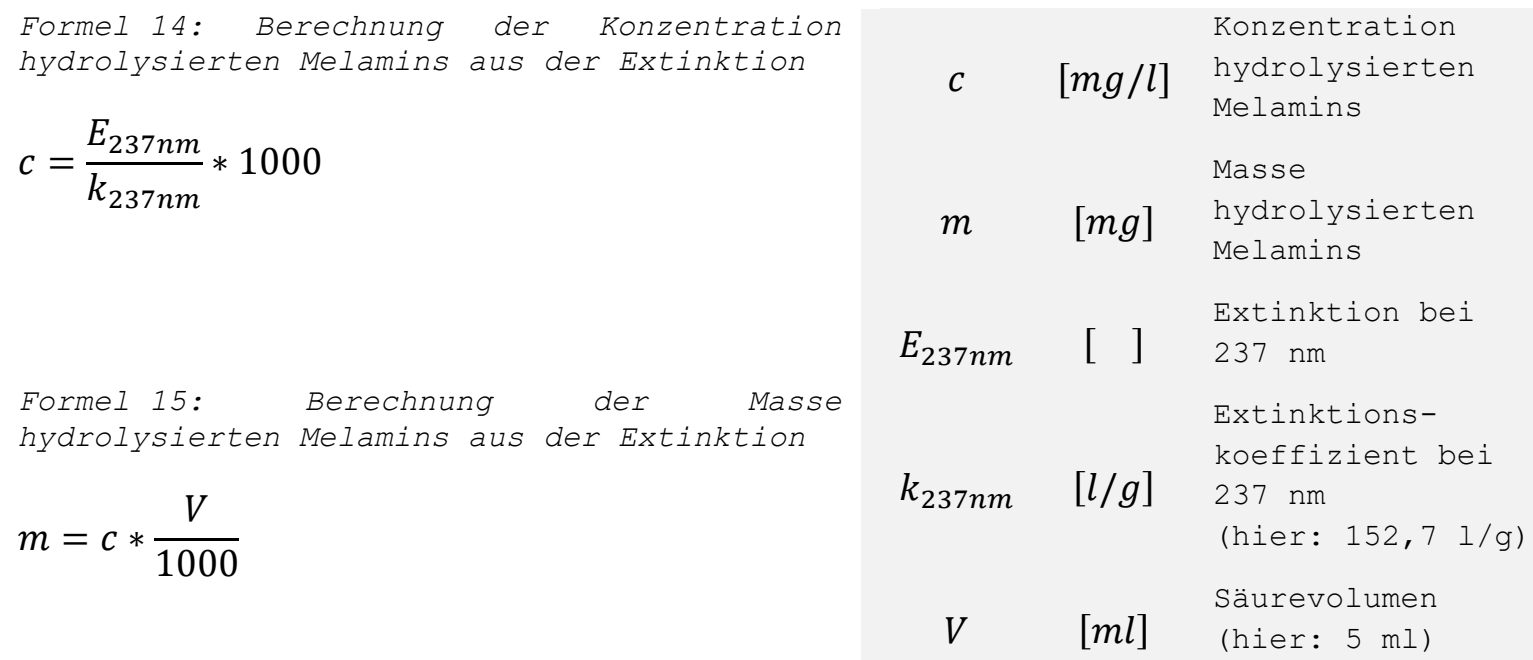

Tabelle 16: Parameter zur Messung des Aushärtungsgrades mittels Pulverhydrolyse

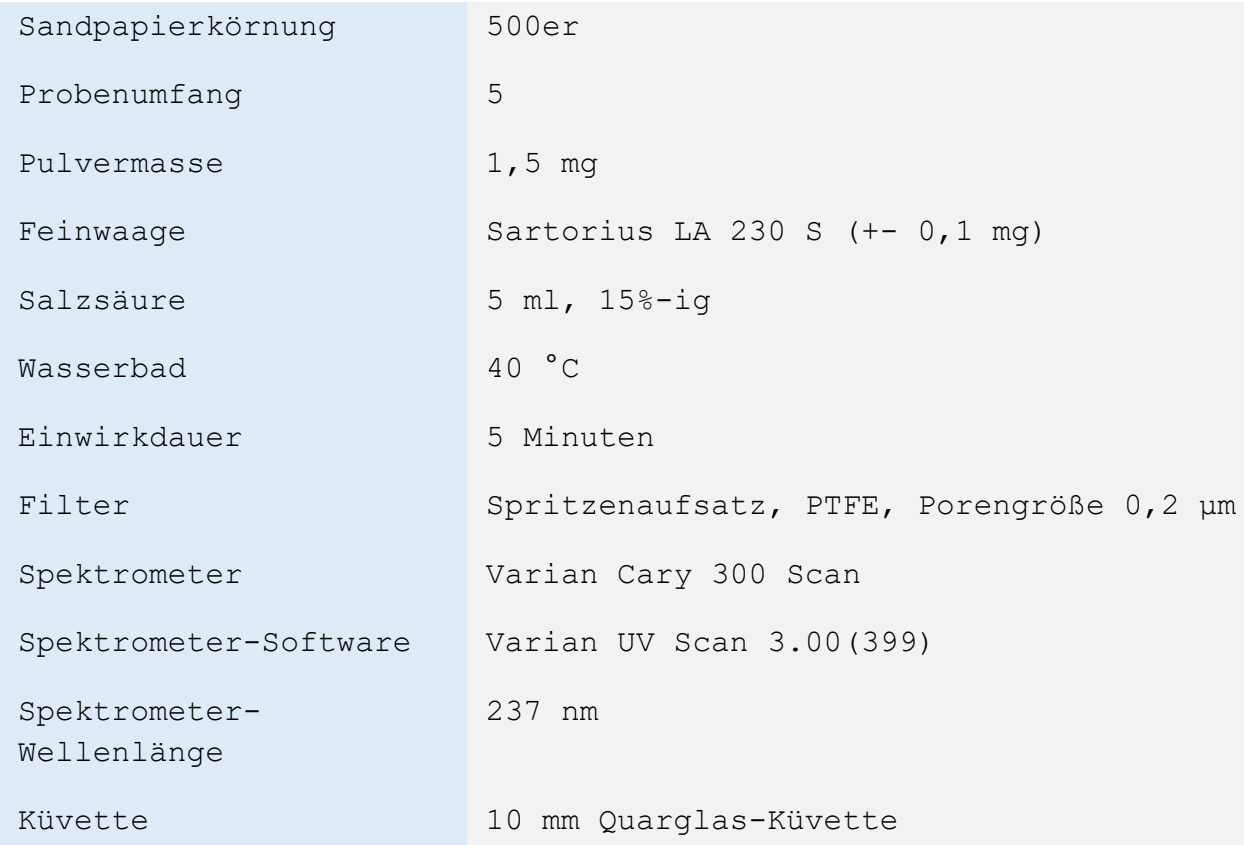




\subsubsection{AUSHÄRTUNGSGRAD MITTELS KITON-TEST}

Beim Kiton-Test wird die Direktbeschichtung einer Säurelösung ausgesetzt. Es wird jedoch nicht hydrolysiertes Melamin quantifiziert, sondern die Oberflächenveränderung klassifiziert.

Der Kiton-Test wird gemäß der Prüfvorschrift des Fraunhofer WKI durchgeführt (Anonymus ) ${ }^{1}$. Eine Farblösung wirkt unter einer Uhrglasschale abgedeckt auf die Prüfoberfläche ein (Tabelle 17). Anschließend wird die Oberfläche mit Wasser gesäubert und der Verfärbung durch die Prüflösung eine von sechs sogenannten Anfärbungsstufen zugewiesen: keine Anfärbung (Überhärtung) bis starke Verfärbung (Unterhärtung).

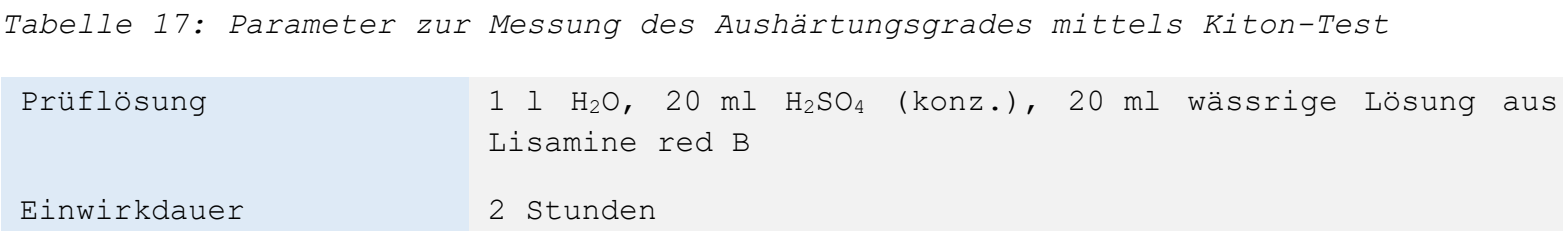

\subsubsection{MIKROSKOPIE}

Aufnahmen werden mittels Auflichtmikroskopie angefertigt. Querschnitte werden vorher mit Schleifpapier (Körnung P500, P1000, dann P3000) geschliffen. Risse werden gegebenenfalls vor dem Schleifen eingefärbt. Dazu werden die Risse mit Tuschierpaste eingerieben und anschließend mit Ethanol gereinigt (Tabelle 18). Die Farbe dringt in die Risse ein und ist auch nach der Reinigung sichtbar. Teilweise werden Proben werden fluoreszenzmikroskopisch (Anregung bei $365 \mathrm{~nm}$ ) dokumentiert.

Tabelle 18: Parameter zur mikroskopischen Untersuchung

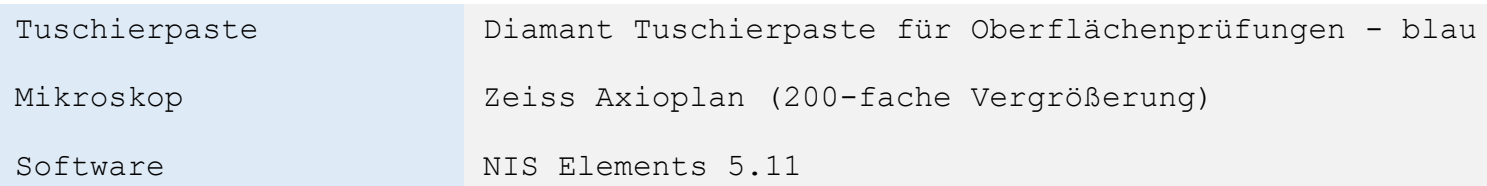

\subsubsection{MATERIALFEUCHTE}

Materialfeuchten werden nach der Darrtrocknungsmethode (DIN EN 13183-1-Ber:2003) bestimmt. Von Proben wird die Masse mit einer Waage vor und nach einer Trocknung aufgenommen (Tabelle 19). Die Materialfeuchte berechnet sich nach Formel 16.

\begin{tabular}{|c|c|c|c|}
\hline Formel 16: Materialfeuchte & $u$ & {$[\%]$} & Materialfeuchte \\
\hline$u=\frac{\left(m_{\text {feucht }}-m_{\text {trocken }}\right)}{m} * 100$ & $m_{\text {feucht }}$ & {$[g]$} & Masse der \\
\hline & $m_{\text {trocken }}$ & {$[g]$} & $\begin{array}{ll}\text { Masse der } & \text { der } \\
\text { trockenen Probe }\end{array}$ \\
\hline
\end{tabular}

\footnotetext{
${ }^{1}$ Die Norm AS/NZS 4266.21:2004 beschreibt ein ähnliches Verfahren, welches sich im Wesentlichen nur beim Färbemittel (Rhodamine) und der Evaluierung unterscheidet. In der Norm gibt es nur 5 Klassen. Zusätzlich zur Verfärbung wird auch ein möglicher Glanzverlust evaluiert.
} 
Tabelle 19: Parameter zur Messung der Materialfeuchte

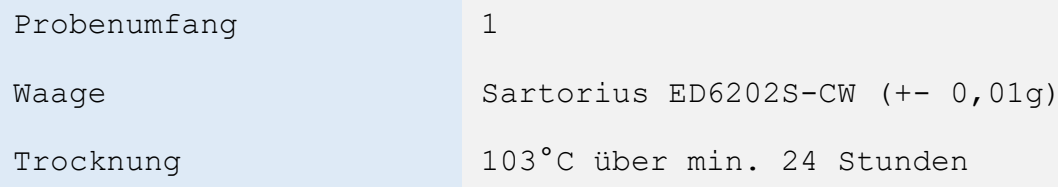

\subsubsection{THERMOGRAFIE}

Für Thermografie-Aufnahmen wurde die Kamera FLIR B20 HS PAL verwendet.

\subsection{StATISTISCHE AUSWERTUNGEN}

\subsubsection{NORMALVERTEILUNG}

Messwerte werden auf Normalverteilung mittels Wahrscheinlichkeitsnetz überprüft. Sämtliche Messwerte werden der Größe nach sortiert und erhalten eine kumulierte Häufigkeit nach Formel 17. Die Messwerte werden mit ihrem Wert als Y-Ordinate und der kumulierten Häufigkeit als X-Ordinate in ein Koordinatensystem eingetragen. Liegt eine Normalverteilung der Werte vor, so ergeben die Punkte näherungsweise eine Gerade.

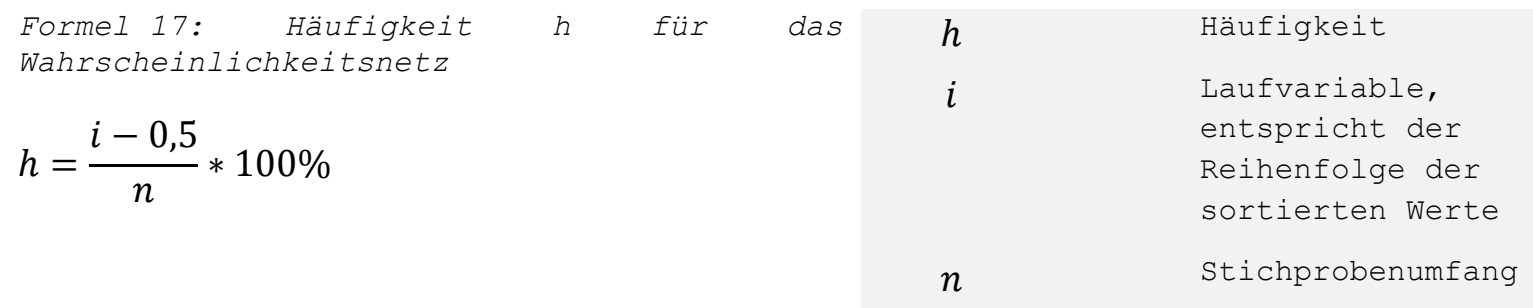

\subsubsection{AUSREIßER}

Ausreißer werden nach GRUBBS 1969 identifiziert. Dazu wird die maximale Differenz zwischen den Einzelwerten einer Gruppe und deren Mittelwert ins Verhältnis gesetzt zur Standardabweichung (Formel 18). Ist dieses Verhältnis (g-Value) größer als ein vorgegebener Grenzwert, gilt der Einzelwert, der die maximale Differenz zum Mittelwert aufweist, als Ausreißer. Die Grenzwerte gibt GRUBBS 1969 in Abhängigkeit vom Stichprobenumfang der Gruppe und dem Vertrauensniveau vor.

$$
\begin{aligned}
& \text { Formel 18: g-Value nach Grubbs } \\
& g=\frac{\max _{i}\left|x_{i}-\bar{x}\right|}{s}
\end{aligned}
$$

$\begin{array}{ll}g & \text { g-Value } \\ \bar{x} & \text { Mittelwert } \\ x_{i} & \text { Einzelwert } i \\ s & \text { Standardabweichung }\end{array}$




\subsubsection{MitTELWERTVERGLEICH}

Einzelne Gruppen wurden auf signifikante Unterschiede untersucht, indem das Vertrauensniveau der Mittelwertdifferenz geprüft wird (Formel 19). Liegt zwischen den Grenzen des Vertrauensniveaus der Wert 0, unterscheiden sich die Gruppen für das gewählte Vertrauensniveau nicht signifikant.

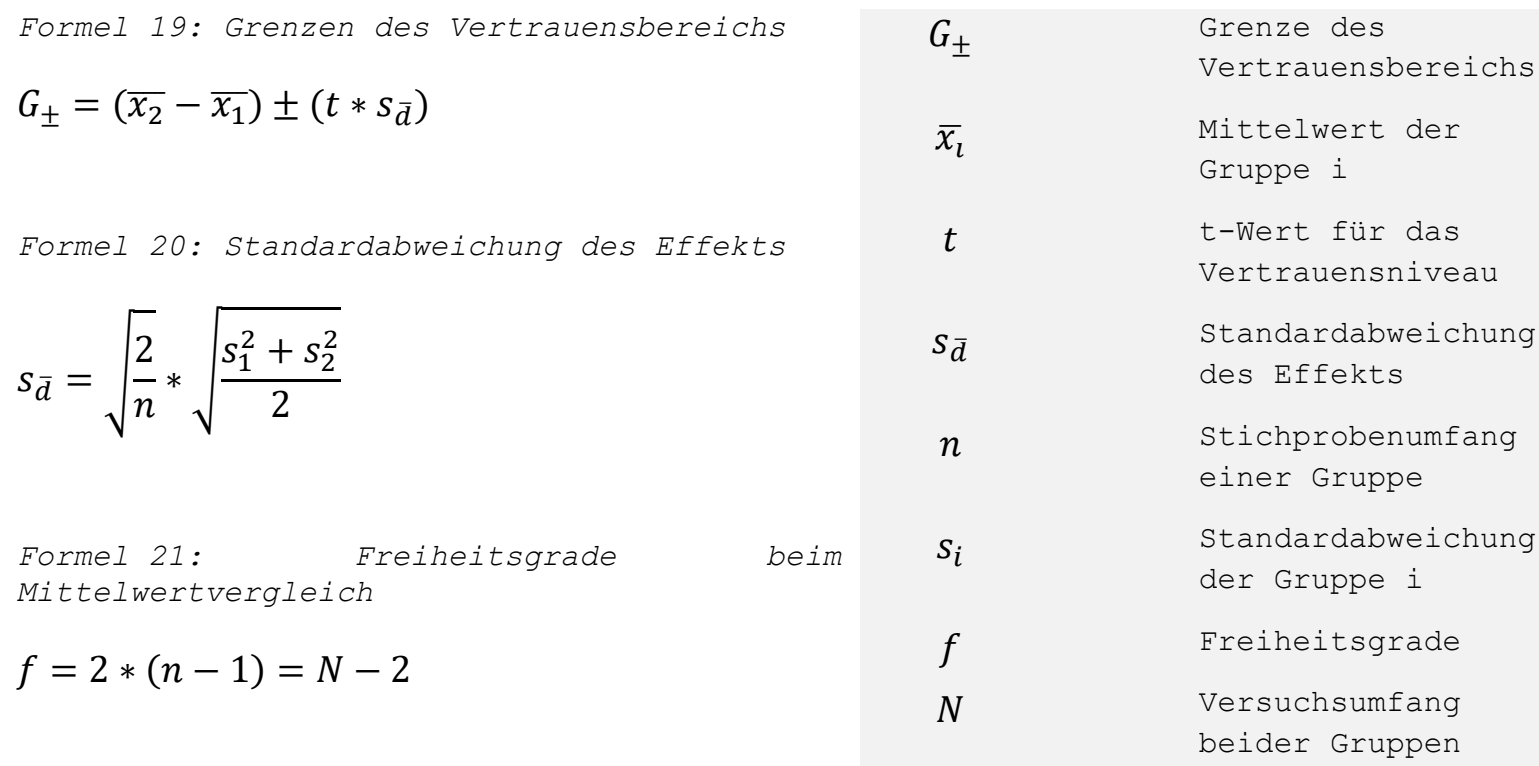

$G_{ \pm}$

$\bar{x}_{l}$

$t$

$S_{\bar{d}}$

$n$

$S_{i}$

Mittelwertvergleich

$f=2 *(n-1)=N-2$

Freiheitsgrade beim

Grenze des

Vertrauensbereichs

Mittelwert der Gruppe i

$t$-Wert für das

Vertrauensniveau

Standardabweichung des Effekts

Stichprobenumfang einer Gruppe

Standardabweichung der Gruppe i

Freiheitsgrade

Versuchsumfang beider Gruppen

Es werden die t-Werte aus KLEPPMANN (2016) verwendet. Die dazu notwendigen Freiheitsgrade können nach Formel 21 berechnet werden. Als Vertrauensniveau wird sowohl 95\% (schwach signifikant), 99\% als auch 99,9\% (hoch signifikant) überprüft.

\subsubsection{ANOVA}

Um Modelle auf signifikante Einflüsse zu prüfen, werden ANOVAs (Analysis of variance) angewendet. Die Berechnungen und Auswertungen erfolgt mittels der Software Design Expert 10 (Stat-Ease, Inc.). Die Modelle sind dem Anhang beigefügt. 


\subsection{UNTERSUCHUNGEN VON METHODEN}

\subsubsection{RISSANFÄLLIGKEIT}

DIN EN 14323 (2014) definiert eine Prüfung des Risswiderstands von Direktbeschichtungen. Um die Differenzierbarkeit zu erhöhen, wurde die Normprüfung modifiziert. Im Folgenden sind die Untersuchungen dargestellt, die zur Entwicklung der Modifikation, genannt Rissanfälligkeitsprüfung, beigetragen haben.

Es sind nur die Modifikationen zur in Kapitel 3.3.1 dargestellten Fertigentwicklung aufgelistet. Sämtliche Untersuchungen wurden an Probensätzen bestehend aus jeweils 3 Proben jeder industriell beschichteten Platte (Tabelle 20) durchgeführt. Als Ergebnis werden teilweise die Ergebnisse zu den einzelnen Platten und teilweise das arithmetische Mittel des Probensatzes angegeben.

Tabelle 20: Auflistung der 8 industriell beschichteten Platten.

Bei der "alternativen" Spanplatte handelt es sich um die l6er-Spanplatte mit feiner Deckschicht (Kapitel 3.1.3)

\begin{tabular}{|c|c|c|c|c|}
\hline Platte & Reaktivität des Harzes & Trägerplatte & Presszeit & Blättrigkeit \\
\hline A & Langsam & Alternative & $38 \mathrm{~s}$ & 1 \\
\hline B & Langsam & Alternative & $55 \mathrm{~s}$ & 1 \\
\hline $\mathrm{C}$ & Schnell & Alternative & $38 \mathrm{~s}$ & 1 \\
\hline $\mathrm{D}$ & Langsam & Alternative & $55 \mathrm{~s}$ & 2 \\
\hline $\mathrm{E}$ & Langsam & Standard & $38 \mathrm{~s}$ & 1 \\
\hline $\mathrm{F}$ & Langsam & Standard & $55 \mathrm{~s}$ & 1 \\
\hline G & Schnell & Standard & $38 \mathrm{~s}$ & 1 \\
\hline $\mathrm{H}$ & Langsam & Standard & $38 \mathrm{~s}$ & 2 \\
\hline
\end{tabular}

\section{4-STÜNDIGE WARTEPHASE}

Die Normprüfung sieht zwischen Ofenbelastung und Evaluierung eine 24-stündige Abkühlphase vor. Bei mehreren Probensätzen wurde sowohl direkt nach der Ofenbelastung als auch nach der Abkühlphase die Rissanfälligkeit bestimmt.

\section{INDIKATORWAHL FÜR DIE RISSANFÄLLIGKEIT}

Von einem Probensatz wird die Rissanfälligkeit an Hand der Anzahl der Risse sowie der maximalen, kumulierten und mittleren Risslänge ausgewertet.

\section{WAHL DER FEHLSTELLEN UND EINSEITIGE BZW. BEIDSEITIGE PRÜFUNG}

In mehrere Probensätze werden Nuten und Bohrlöcher unterschiedlicher Formen und Größen als Rissinitialen eingebracht.

\section{EINSEITIGE BZW. BEIDSEITIGE PRÜFUNG}

Ein Probensatz erhält nur auf der Vorder-, einer nur auf der Rückseite und einer auf beiden Seiten Rissinitialen. 


\section{TEMPERATUR UND DAUER DER OFENBELASTUNG}

Ofentemperatur und Belastungsdauer werden variiert. Für jeden Messpunkt (Temperatur und Dauer) wird ein Probensatz geprüft. Von den Proben wird die Materialfeuchte vor und nach der Ofenbelastung gemäß Darrmethode (Kapitel 3.3.17) bestimmt. Nach Formel 22 wird der Einfluss der relativen Luftfeuchte des Ausgangsklimas auf die relative Luftfeuchte im Ofen berechnet.

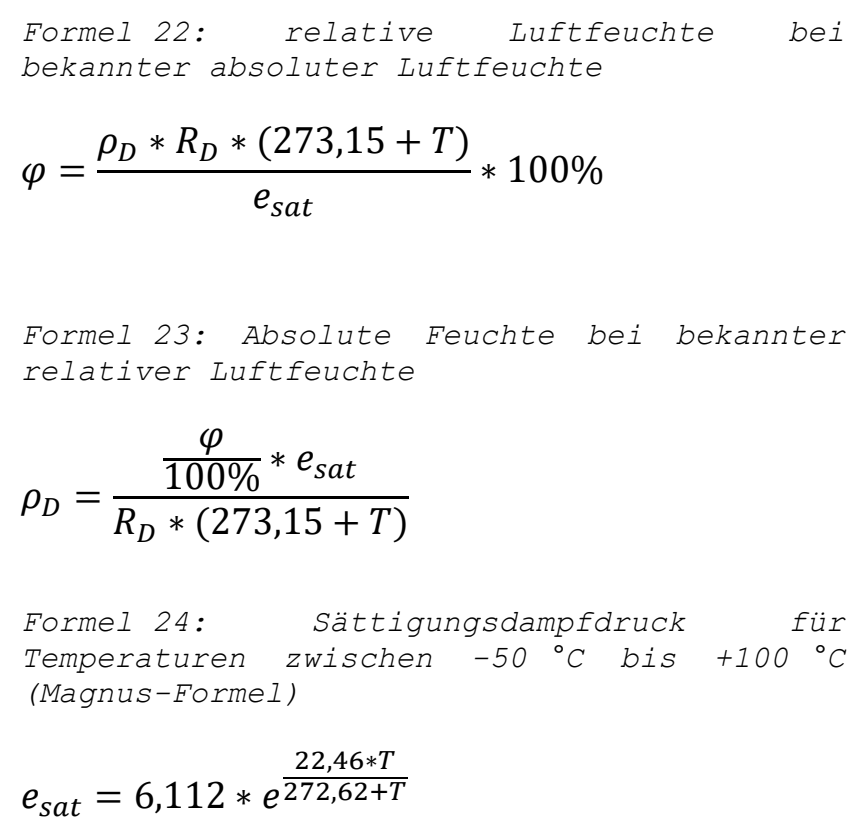

\subsubsection{PULVERHYDROLYSE}

Die Hydrolyse-Methoden zur Bestimmung des Aushärtungsgrads basieren auf einer verbreiteten Methode (Morath; Woods 1958; Nowak-Ossorio; Braun 1982; SChindlbaUer; Anderer 1980). Das Verfahren wurde zur Pulverhydrolyse (Kapitel 3.3.15) weiterentwickelt. Im Folgenden sind Untersuchungen zu dieser Methode dargestellt.

\section{UV-SPEKTREN UND MELAMINKONZENTRATION}

Von Harnstoff, Melamin und Dissoziationsprodukten von Melamin werden Konzentrationsreihen in Salzsäure angefertigt und diese UV-spektrometrisch von $190 \mathrm{~nm}$ bis $900 \mathrm{~nm}$ vermessen.

Aus der Konzentrationsreihe von Melamin wird mittels linearer Regression eine Formel aufgestellt, um von der gemessenen Extinktion bei $237 \mathrm{~nm}$ auf die Melaminkonzentration schließen zu können.

\section{ZEITLICHE VERÄNDERUNGEN DES SPEKTRUMS}

Von der industriell beschichteten Platte $C$ (Kapitel 3.5.1) wird eine Hydrolyselösung hergestellt. Das UV-Spektrum der Lösung wird direkt nach der Hydrolyse und nach mehrstündiger Lagerung aufgenommen. Nach 4 Tagen wird die Probe vermessen, geschüttelt und ein letztes Mal vermessen.

\section{UMGEBUNGSTEMPERATUR, HYDROLYSEDAUER UND SÄUREKONZENTRATION}

Der Aushärtungsgrad der industriell beschichteten Platte $\mathrm{C}$ wird bei unterschiedlichen Temperaturen, Säurekonzentrationen und Hydrolysezeiten bestimmt. 


\section{REINIGUNGSMITTEL}

Jeweils 8 Proben der industriell beschichteten Platte $C$ (Kapitel 3.5.1) werden mit Ethanol, Wasser, Aceton oder einem Papier gereinigt und anschließend deren Aushärtungsgrad bestimmt.

\section{VERGLEICH MIT DEM KITON-TEST}

Von 9 Platte aus der Versuchsreihe „Aushärtungsbedingungen“ (Kapitel 3.6.1) wird der Aushärtungsgrad nach der Pulverhydrolyse und nach dem Kiton-Test (Kapitel 3.3.19) bestimmt. Die Platten wurden mit Imprägnaten des schnell härtenden Harzes mit kaltem Pressblech beschichtet. Pressdauer und -temperatur werden variiert, um unterschiedliche Aushärtungsgrade einzustellen (Kapitel 3.6.1).

\subsubsection{Model ZUR BereChNUNG MeChANISCHER KenNWERTE DER DiReKTBESCHICHTUNG}

Um den Einfluss der Eingabewerte auf das Model evaluieren zu können, wurde eine Sensitivitätsanalyse durchgeführt. Jeweils ein Eingabewert wurde variiert und der sich dadurch ergebende Einfluss auf Festigkeit und Elastizitätsmodul dokumentiert. 


\subsection{VERSUCHSREIHEN ZU EINFLUSSGRÖßEN}

\subsubsection{VERSUCHSREIHE: AUSHÄRTUNGSBEDINGUNGEN}

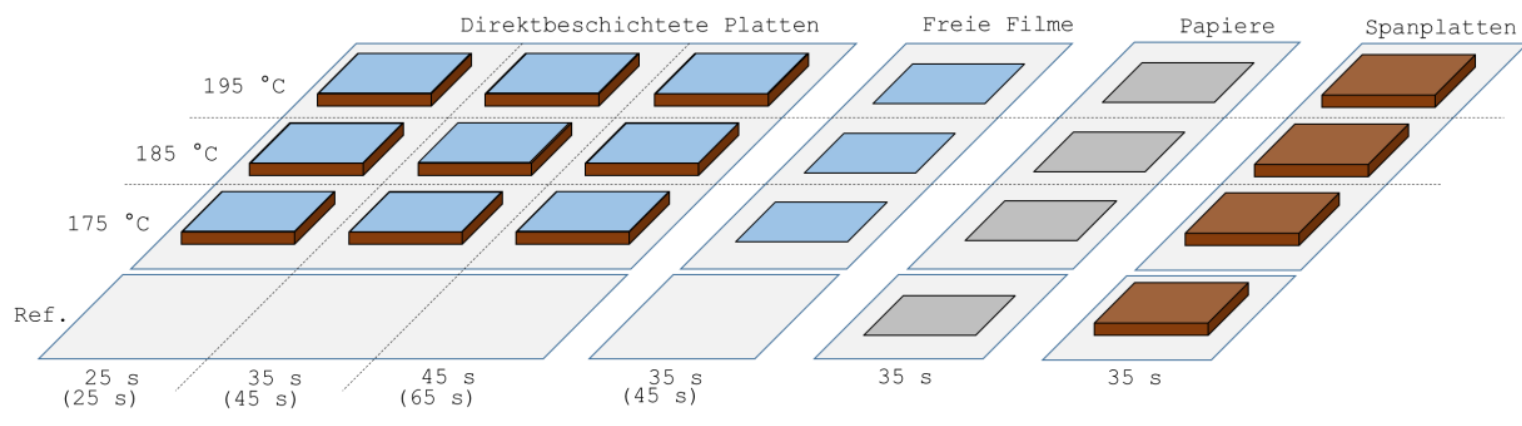

Abbildung 15: Hergestelltes Prüfmaterial zur Untersuchung der Auswirkungen von Aushärtungsbedingungen.

Bei den direktbeschichteten Platten und freien Filmen stehen die Presszeiten des langsam härtenden Harzes in Klammern und die des schnell härtenden Harzes darüber.

Ziel dieser Versuchsreihe war es, die Einflüsse der Harzreaktivität, Presstemperatur und Pressdauer auf die Rissbildung zu prüfen. Untersucht wurden direktbeschichtete Platten, freie Filme, Papiere und Spanplatten (Abbildung 15). Tabelle 21 listet die Untersuchungen auf, die an dem Material durchgeführt wurden.

\section{DIREKTBESCHICHTETE PLATTEN}

Es wurden Spanplatten (16 mm Standardplatte) mit Imprägnaten beider Harze (gefülltes Standardpapier) beschichtet. Imprägnate des langsam härtenden Harzes wurden mit kalten Pressblechen beschichtet. Vom schnell härtenden Harz wurde Imprägnate mit kalten und mit vorgeheizten Pressblechen beschichtet. Mit jeder der drei Kombinationen aus Harzreaktivität und Pressverfahren wurden 9 Platten mit unterschiedlicher Pressdauer und -Temperatur direktbeschichtet. Als Temperatur wurden die vom Imprägnierer empfohlenen $185^{\circ} \mathrm{C}$ und zusätzlich $175^{\circ} \mathrm{C}$ und $195^{\circ} \mathrm{C}$ eingestellt. Die Pressdauer wurde an das Harz angepasst. Das langsam härtende Harz wurde $25 \mathrm{~s}$, $45 \mathrm{~s}$ (Empfehlung des Imprägnierers) und $65 \mathrm{~s}$ verpresst, das schnell härtende Harz $25 \mathrm{~s}, 35 \mathrm{~s}$ (Empfehlung des Imprägnierers) und $45 \mathrm{~s}$.

\section{FREIE FILME}

Von allen vier Imprägnaten wurden freie Filme produziert. Die Presstemperatur wurde analog zu den direktbeschichteten Platten variiert $\left(175^{\circ} \mathrm{C}, 185^{\circ} \mathrm{C}\right.$ und $\left.195^{\circ} \mathrm{C}\right)$. Es wurden die Empfehlungen zur Pressdauer des Imprägnierers für das jeweilige Harz eingehalten und nicht variiert (35 s für das schnell härtende und $45 \mathrm{~s}$ für das langsam härtende Harz).

\section{SPANPLATTEN UND NICHT IMPRÄGNIERTE PAPIERE}

Spanplatten (16 mm Standardplatte) und nicht-imprägnierte Papiere (gefülltes Standardpapier) wurden mit vorgeheizten Pressblechen heißverpresst. Die Presstemperatur wurde variiert $\left(175^{\circ} \mathrm{C}\right.$, $185^{\circ} \mathrm{C}, 195^{\circ} \mathrm{C}$ ), die Dauer lag bei $35 \mathrm{~s}$. Eine Spanplatte und ein Papier wurden als Referenz nicht erneut heißverpresst. 


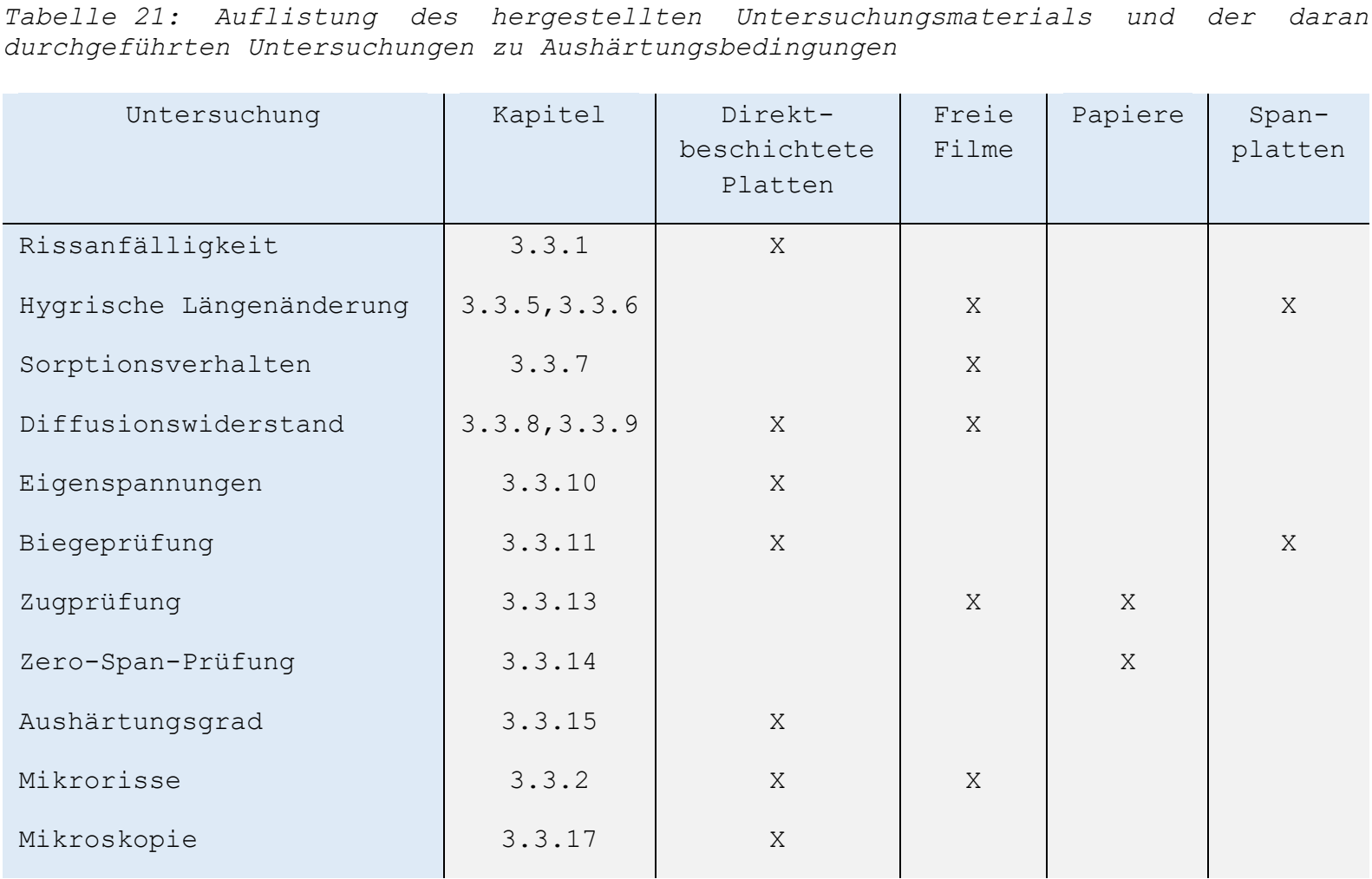

\subsubsection{VeRSUCHSREIHE: BLÄTtRIGKEIT UND FüLLGRAD DES PAPIERS}

Die Blättrigkeit und der Füllgrad des Papiers sollten variiert und somit deren Einflüsse auf die Rissbildung untersucht werden. Als Blättrigkeit versteht man die Anzahl an Imprägnatlagen einer Direktbeschichtung.

Es wurden 32 direktbeschichtete Platten aus allen Kombinationen der folgenden Optionen mit kaltem Pressblech verpresst:

- Trägerplatte: Standard-Spanplatte oder alternative $16 \mathrm{~mm}$ Spanplatte

- Pressdauer: $48 \mathrm{~s}$ oder $65 \mathrm{~s}$

- Harz: langsam oder schnell härtendes Harz

- Imprägnierpapier: gefülltes Standardpapier oder niedriger gefülltes Alternativpapier

- Blättrigkeit: 1 oder 2 (parallel übereinanderliegende) Imprägnate

Zusätzlich zu den Platten wurden 1-blättrige und 2-blättrige freie Filme produziert (schnell härtendes Harz, gefülltes Standardpapier). Tabelle 22 listet die Methoden auf, die an dem so hergestellten Material durchgeführt wurden².

${ }^{2}$ Der Versuch wurde ursprünglich als vollfaktorieller Versuchsplan entworfen, um die Einflüsse der fünf Optionen zu bestimmen. Die Einflüsse von Trägerplatten, Pressdauer sowie Harzreaktivität wurden später als separate Versuchsreihen ausgelagert. In dieser Versuchsreihe wird daher auf die Prüfung der Einflüsse dieser Optionen verzichtet. 
Tabelle 22: Auflistung des hergestellten Untersuchungsmaterials und der daran durchgeführten Untersuchungen zur Blättrigkeit

\begin{tabular}{l|c|c|c}
\multicolumn{1}{c|}{ Untersuchung } & Kapitel & Direktbeschichtete Platten & $\begin{array}{c}\text { Freie } \\
\text { Filme }\end{array}$ \\
\hline Rissanfälligkeit & 3.3 .1 & $\mathrm{X}$ & \\
Hygrische Längenänderung & 3.3 .6 & & $\mathrm{X}$ \\
Sorptionsverhalten & 3.3 .7 & $\mathrm{X}$ \\
Zugprüfung & 3.3 .13 & $\mathrm{X}$ \\
Aushärtungsgrad & 3.3 .15 & $\mathrm{X}$ & $\mathrm{X}$ \\
Mikrorisse & 3.3 .2 & $\mathrm{X}$
\end{tabular}

\subsubsection{VeRSUCHSREIHE: MATERIALFEUCHTE}

Es sollte geprüft werden, ob die Materialfeuchte von Spanplatte und Imprägnat die Rissanfälligkeit der direktbeschichteten Platte beeinflussen. Spanplatten (16 mm Standardplatte) und Imprägnate des schnell härtenden Harzes (gefülltes Standardpapier) wurden bei $20^{\circ} \mathrm{C}$ und $30 \%, 50 \%$ oder $65 \%$ rel. Lf. über 8 Wochen klimatisiert. Anschließend wurden alle neun möglichen Kombinationen aus den drei Spanplatten- und den drei Imprägnatsfeuchten verpresst. Unmittelbar vor dem Verpressen wurden von Abschnitten der Spanplatte und der Imprägnate die Feuchte nach der Darrmethode (Kapitel 3.3.18) bestimmt. Tabelle 23 listet die Methoden auf, die an dem Material durchgeführt wurden.

Tabelle 23: Auflistung des hergestellten Untersuchungsmaterials und der daran durchgeführten Untersuchungen zu Materialfeuchten

\begin{tabular}{l|c|c}
\multicolumn{1}{c|}{ Untersuchung } & Kapitel & Direktbeschichtete Platten \\
\hline Rissanfäligkeit & 3.3 .1 & $\mathrm{X}$ \\
Eigenspannungen & 3.3 .10 & $\mathrm{x}$ \\
Mikrorisse & 3.3 .2 & $\mathrm{x}$ \\
Mikroskopie & 3.3 .17 & $\mathrm{x}$
\end{tabular}




\subsubsection{VERSUCHSREIHE: OBERFLÄCHENDEFEKTE}

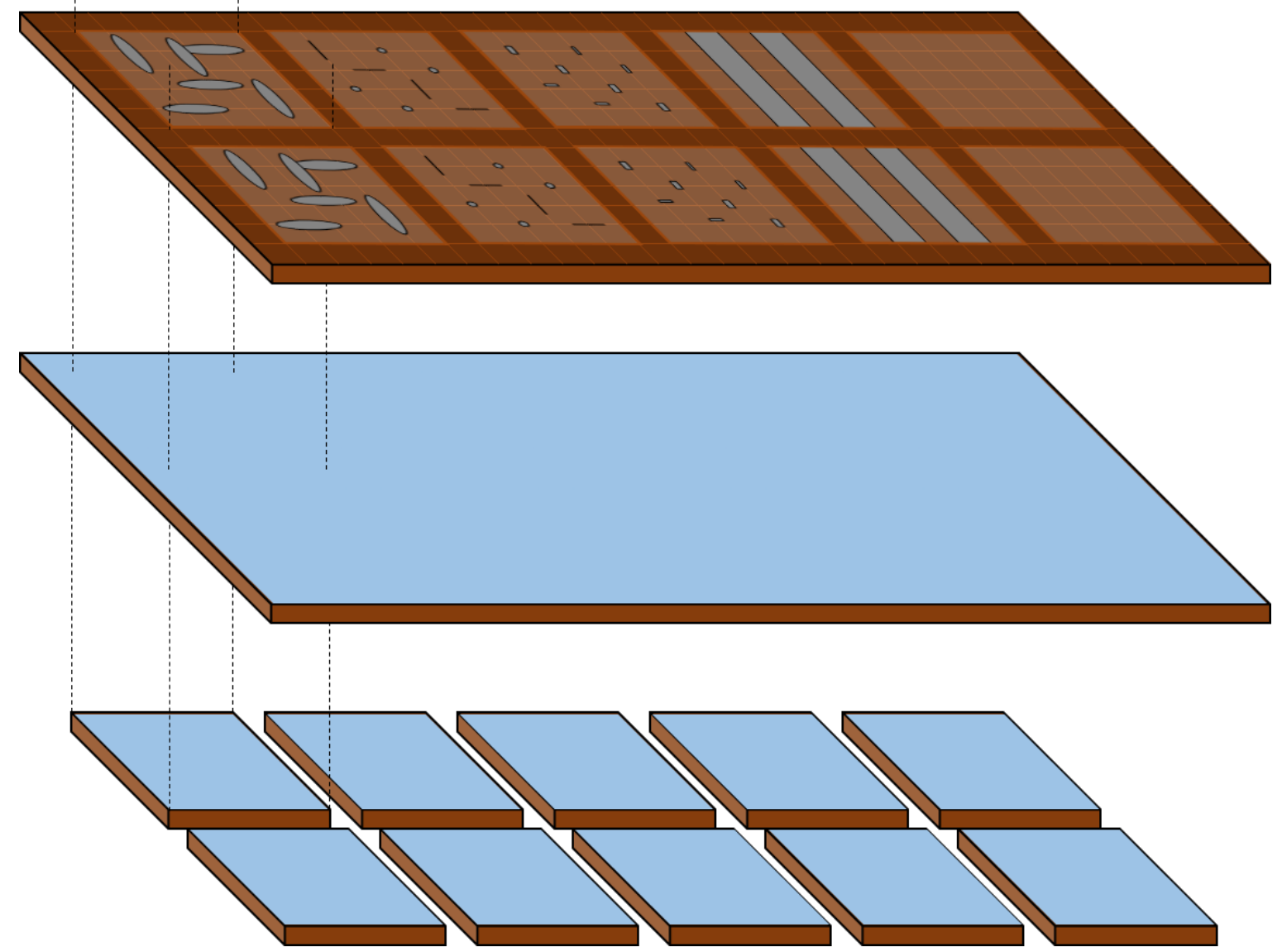

Abbildung 16: Lage der einzelnen Oberflächendefekte auf den Plattenhälften

Oben: unbeschichtete Platte mit eingebrachten Defekten, Mitte: beschichtete Platte mit Defekten in der Spanplatte, Unten: Ausgesägte Proben der beschichteten Platte.

Aus Schadensanalysen ist bekannt, dass Störstellen in der Spanplattendeckschicht zu Rissen in der darüber liegenden Direktbeschichtung führen können. Es sollte überprüft werden, bei welchen Defekten es zu einer solchen Rissbildung kommt. Dazu wurde eine Spanplatte (16 mm Standardplatte) längs halbiert. Auf jede Plattenhälfte wurde ein $5 \mathrm{~cm}$-Raster gezeichnet und 10 Bereiche mit $25 \mathrm{~cm} \times 25 \mathrm{~cm}$ markiert. Diese Flächen wurden mit unterschiedlichen Oberflächendefekte versehen (Abbildung 16, von links nach rechts angeordnet):

1. Mit einem schräg gehaltenem Handschleifgerät wird an mehreren Flächen die Oberfläche abgeschliffen.

2. Mit einem Cutter-Messer wird mit unterschiedlichem Druck in die Deckschicht geschnitten. Zusätzlich werden mit Hammer und Nagel Störstellen eingeschlagen.

3. Mehrere längliche Löcher werden mit einer Oberfräse eingebracht.

4. An einem $5 \mathrm{~cm}$ breiten Bereich wird mit einer Pinzette einzelne Fasern der Deckschicht entfernt und in einem weiteren $5 \mathrm{~cm}$ breiten Abschnitt werden lose Fasern mit einem Klebeband abgezogen.

5. Die Oberfläche wird als Referenzprobe nicht mit Störstellen versehen.

Die Plattenhälften wurden mit Imprägnaten des schnell härtenden Harzes (gefülltes Standardpapier) direktbeschichtet. Eine Hälfte wurde $35 \mathrm{~s}$ lang verpresst, die andere $45 \mathrm{~s}$. Anschließend wurde das $5 \mathrm{~cm}$-Raster auf den Platten nachgezeichnet und die $25 \mathrm{~cm} \times 25 \mathrm{~cm}$ Bereiche rausgesägt. Tabelle 24 listet die Methoden auf, die an dem so hergestellten Material durchgeführt wurden. Die 
Rissanfälligkeitsmethode wurde abweichend zur in Kapitel 3.3.1 beschrieben Methode ohne Nuten und Bohrlöcher durchgeführt.

Tabelle 24: Auflistung des hergestellten Untersuchungsmaterials und der daran durchgeführten Untersuchungen zu Oberflächendefekten

\begin{tabular}{l|c|c}
\multicolumn{1}{c|}{ Untersuchung } & Kapitel & Direktbeschichtete Platten \\
\hline Rissanfälligkeit & $\begin{array}{c}\text { Abwandlung } \\
\text { zu } 3.3 .1\end{array}$ & $\mathrm{X}$ \\
Aushärtungsgrad & 3.3 .15 & $\mathrm{X}$ \\
Mikroskopie & 3.3 .17 & $\mathrm{X}$
\end{tabular}

\subsubsection{VERSUCHSREIHE: TRÄGERPLATTEN}

Bei dieser Versuchsreihe sollte der Einfluss der Trägerplatten auf die Rissbildung untersucht werden.

Auf verschiedene bei $20^{\circ} \mathrm{C} / 50 \%$ rel. Lf. klimatisierte Trägerplatten (Kapitel 3.1.3) wurden Imprägnate des schnell härtenden Harzes (gefülltes Standardpapier) mit vorgeheizten Pressblechen verpresst. Die Presse wurde an die unterschiedlichen Plattenstärken angepasst. Tabelle 25 listet die Methoden auf, die an dem so hergestellten Material durchgeführt wurden.

Tabelle 25: Auflistung des hergestellten Untersuchungsmaterials und der daran durchgeführten Untersuchungen zu Trägerplatten

\begin{tabular}{l|c|c}
\multicolumn{1}{c|}{ Untersuchung } & Kapitel & Direktbeschichtete Platten \\
\hline Rissanfälligkeit & 3.3 .1 & $\mathrm{X}$ \\
Makrorissprojektion & 3.3 .3 & $\mathrm{X}$ \\
Mikrorissprojektion & 3.3 .4 & $\mathrm{X}$ \\
Eigenspannungen & 3.3 .10 & $\mathrm{X}$ \\
Mikrorisse & 3.3 .2 & $\mathrm{X}$ \\
Mikroskopie & 3.3 .17 & $\mathrm{X}$
\end{tabular}




\section{ERgEBNISSE \& DISKUSSION ZU UNTERSUCHUNGEN VON METHOdEN}

\subsection{RISSANFÄLLIGKEITSPRÜFUNG}

Die Normprüfung DIN EN 14323 (2014) erlaubt eine Klassifizierung des Risswiderstands. Um die Differenzierbarkeit zu erhöhen, wurden zahlreiche Modifikationen geprüft.

\subsubsection{EINFLUSS DER WARTEPHASE}

Die Normprüfung sieht eine 24-stündige Wartephase zwischen der Ofenbelastung und der Evaluierung vor. Es wurde geprüft, ob sich das Rissbild währenddessen signifikant ändert oder die Wartephase entfallen kann.

\section{ERGEBNISSE}

Während der Wartephase kam es bei 2 von insgesamt 144 geprüften Proben zu Veränderungen. Bei beiden Platten war jeweils 1 Riss um 0,5 mm länger geworden.

\section{DISKUSSION}

Auf Grund des marginalen Unterschieds der kumulierten Risslängen wurde bei weiteren Prüfungen von der Wartephase abgesehen. Bei weiteren Untersuchungen erfolgt die Evaluierung unmittelbar nach dem Abkühlen der Platten.

\subsubsection{INDIKATORWAHL FÜR DIE RISSANFÄLLIGKEIT}

Bei der Normprüfung wird die Rissbildung klassifiziert. Durch Verwendung einer alternativen Bewertungsgrundlage sollte die Differenzierbarkeit der Rissanfälligkeit optimiert werden.

\section{ERGEBNISSE}

Tabelle 26: Indikatoren als Maß für die Rissanfälligkeit direktbeschichteter Platten

\begin{tabular}{|c|c|c|c|c|c|c|c|c|}
\hline \multirow[t]{2}{*}{ Platte } & \multicolumn{2}{|c|}{$\begin{array}{c}\text { Anzahl } \\
\text { aller Risse }\end{array}$} & \multicolumn{2}{|c|}{$\begin{array}{l}\text { Maximale } \\
\text { Risslänge }\end{array}$} & \multicolumn{2}{|c|}{$\begin{array}{l}\text { Kumulierte } \\
\text { Risslänge }\end{array}$} & \multicolumn{2}{|c|}{$\begin{array}{l}\text { Mittlere } \\
\text { Risslänge }\end{array}$} \\
\hline & [ ] & {$[\%]$} & {$[\mathrm{mm}]$} & {$[\%]$} & {$[\mathrm{mm}]$} & {$[\%]$} & {$[\mathrm{mm}]$} & {$[\%]$} \\
\hline A & 10 & 9 & 2 & 3 & 5 & 3 & 1 & 5 \\
\hline B & 12 & 11 & 3 & 5 & 11 & 7 & 1 & 8 \\
\hline C & 16 & 15 & 7 & 12 & 23 & 15 & 3 & 14 \\
\hline D & 11 & 11 & 31 & 52 & 47 & 30 & 6 & 34 \\
\hline $\mathrm{E}$ & 9 & 8 & 2 & 4 & 3 & 2 & 1 & 5 \\
\hline $\mathrm{F}$ & 11 & 10 & 3 & 5 & 4 & 3 & 1 & 6 \\
\hline G & 20 & 18 & 5 & 8 & 30 & 19 & 2 & 11 \\
\hline $\mathrm{H}$ & 19 & 18 & 6 & 11 & 34 & 22 & 3 & 15 \\
\hline$\Sigma$ & 107 & 100 & 59 & 100 & 157 & 100 & 18 & 100 \\
\hline
\end{tabular}

Tabelle 26 listet Indikatoren auf, die als Indikator für die Rissanfälligkeit untersucht wurden. Maximale, kumulierte und mittlere Risslänge zeigen eine ähnliche Einstufung der Rissanfälligkeit und eine hohe Spannweite der Ergebnisse. Die Rissanzahl führt teilweise zu einer unterschiedlichen Einstufung (Platte D) und zeigt eine geringe Spannweite. 


\section{DISKUSSION}

Die Dokumentation der maximalen Risslänge verursacht den geringsten Aufwand. Theoretisch muss nur ein einziger Riss vermessen werden. Bei den anderen Indikatoren ist die Aufnahme aller Risse notwendig. Allerdings werden Platten mit vielen kleinen Rissen anhand der maximalen Risslänge und der mittleren Risslänge als wenig rissanfällig bewertet. Für weitere Untersuchungen wurde die kumulierte Risslänge verwendet. Zusätzlich zur guten Differenzierbarkeit gibt sie das Ausmaß der Rissbildung am besten wieder.

Treten viele Risse auf, kann die Dokumentation äußerst lange dauern. In einem Extremfall wurden 15 Minuten für eine einzelne Probe benötigt. Soll die Methode praktische Anwendung finden, ist eine Beschleunigung der Methode durch eine andere ungenauere, aber dafür schnellere Rissdokumentation zu empfehlen. Beispielsweise könnten die Felder eines auf die Oberfläche aufgetragenen Gitternetzes gezählt werden, in denen Risse vorkommen.

\subsubsection{WAHL DER RISSINITIALEN}

Aus Schadensanalysen ist bekannt, dass von praxistypischen Bearbeitungen wie Nuten und Bohrlöchern vermehrt Risse der Direktbeschichtung ausgehen. Es wurde geprüft, ob solche Rissinitialen in den Prüfkörpern Risse provozieren und die Differenzierbarkeit der Rissanfälligkeit verbessern.

\section{ERGEBNISSE}

Tabelle 27: Kumulierte Risslängen [mm] direktbeschichteter Platten an unterschiedlichen Rissinitialen.

Bei den Nuten ist die Form angegeben (Abbildung 17 und Abbildung 18), bei den Bohrlöchern der Durchmesser.

"mit S.": mit eingedrehter Schraube

\begin{tabular}{|c|c|c|c|c|c|c|c|c|}
\hline \multirow[t]{3}{*}{ Platte } & \multicolumn{8}{|c|}{ Kumulierte Risslänge [mm] } \\
\hline & \multirow{2}{*}{$\begin{array}{l}\text { Abseits } \\
\text { von } \\
\text { Fehl- } \\
\text { stellen }\end{array}$} & \multicolumn{3}{|c|}{ Nut } & \multicolumn{4}{|c|}{ Bohrloch } \\
\hline & & / & + & $\mathrm{x}$ & $3 \mathrm{~mm}$ & $5 \mathrm{~mm}$ & $\begin{array}{c}5 \mathrm{~mm} \\
\text { mit } \mathrm{S}\end{array}$ & $35 \mathrm{~mm}$ \\
\hline A & 0 & 5 & 5 & 10 & 0 & 0 & 5 & 0 \\
\hline B & 0 & 11 & 18 & 15 & 0 & 0 & 14 & 0 \\
\hline C & 0 & 21 & 33 & 38 & 5 & 2 & 31 & 6 \\
\hline $\mathrm{D}$ & 0 & 47 & 74 & 69 & 3 & 0 & 28 & 6 \\
\hline $\mathrm{E}$ & 0 & 3 & 5 & 4 & 0 & 0 & 11 & 0 \\
\hline $\mathrm{F}$ & 0 & 4 & 7 & 8 & 1 & 0 & 13 & 0 \\
\hline G & 0 & 26 & 40 & 42 & 21 & 5 & 21 & 14 \\
\hline $\mathrm{H}$ & 6 & 27 & 47 & 39 & 15 & 8 & 33 & 7 \\
\hline$\Sigma$ & 6 & 143 & 229 & 225 & 44 & 14 & 157 & 33 \\
\hline
\end{tabular}

Es wurde darauf geachtet, dass die Rissinitialen gängigen Bearbeitungen von Direktbeschichtungen entsprechen. Bei 144 Probekörper ist nur ein einziger Riss abseits der Fehlstellen aufgetreten (Tabelle 27). An Böhrlöchern entstehen Risse ringsum (Abbildung 17), bei Nuten stets an den Ecken (Abbildung 18). 


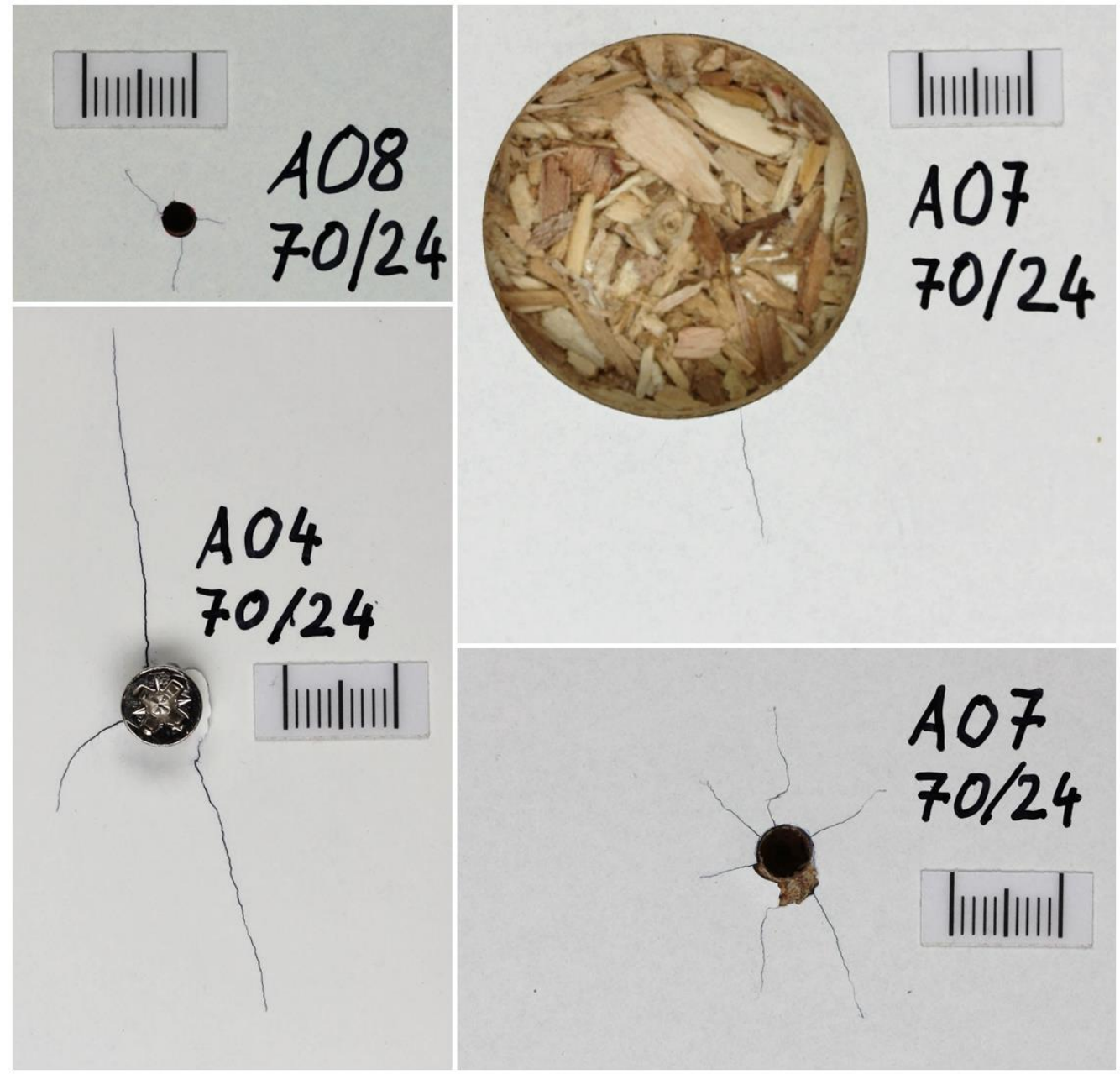

Abbildung 17: Risse an Bohrlöcher bei der Rissanfälligkeitsprüfung.

Es handelt sich um Aufnahmen von Platten unterschiedlicher Rissanfälligkeit. Die Risslängen sind daher nicht vergleichbar.

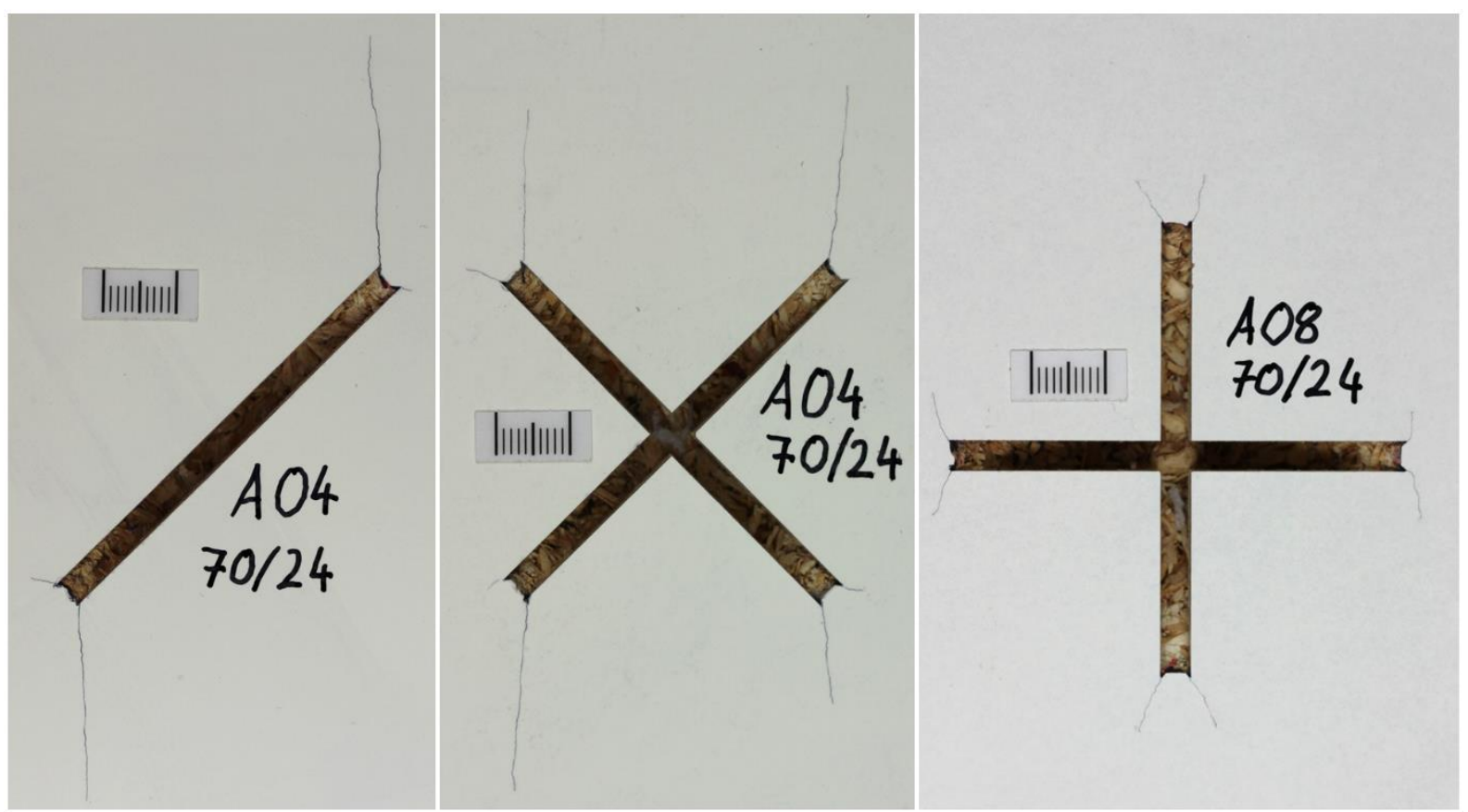

Abbildung 18: Risse an Nuten bei der Rissanfälligkeitsprüfung.

Es handelt sich um Aufnahmen von Platten unterschiedlicher Rissanfälligkeit. Die Risslängen sind daher nicht vergleichbar. 


\section{DISKUSSION}

Die Rissinitialen erlauben eine Differenzierung der Rissanfälligkeit der Platten. Da nur ein einziger Riss abseits der Rissinitialen beobachtet wurde, wäre ohne die Rissinitialen (also bei normgemäßer Prüfung) kein signifikanter Unterschied zwischen den Platten nachweisbar gewesen. In der Praxis sind solche Rissinitialen regelmäßig der Ausgangspunkt von Rissen. Die Rissinitialen erlauben daher eine realistische Differenzierung der Rissanfälligkeit.

An Rissinitialen ergeben sich zwei voneinander leicht abweichende Evaluierungen. An Nuten und Bohrlöchern mit eingedrehter Schraube erlauben die kumulierten Risslängen eine ähnliche Differenzierung. Davon unterscheiden sich die Bohrlöcher ohne Schraube. Deutlich wird dies an Platte D. Platte D hat bei Bohrlöchern ohne Schraube geringe kumulierte Risslängen. An anderen Rissinitialen sind die kumulierten Risslängen dieser Platte hoch, überwiegend sogar die höchsten aller Platten. Erklären lässt sich das mit dem Schwindverhalten der Spanplatte. An den Rissinitialen ist ein Feuchteaustausch der Spanplatte mit der Umgebungsluft gegeben. Die Spanplatte kann während der Ofenbelastung stärker schwinden und die umliegende Direktbeschichtung erfährt höhere Zugspannungen. Bei $3 \mathrm{~mm}$ und $5 \mathrm{~mm}$ Bohrlöchern könnte die Öffnung zu klein sein, um einen nennenswerte Feuchteaustausch auszulösen. Diese Hypothese wird jedoch durch die Risse an der $35 \mathrm{~mm}$ Bohrung entkräftet. Diese sollte mit dem größten Durchmesser besonders deutlich Risse aufgrund der Schwindspannung aufzeigen. Das ist nicht der Fall. Eine weitere Hypothese basiert auf der Spannungsverteilung. Rissinitialen stellen Kerben dar. Kräfte werden um Kerben herumgeleitet, es kommt zu Spannungsspitzen an den Rändern (MATTHECK; BETHGE 2003). Die geometrische Form einer Kerbe spielt bei der Spannungsverteilung eine wichtige Rolle. Bohrungen sind kreisrund, Spannungen werden daher relativ problemlos umgeleitet. Hingegen kommt es bei den Nuten zu Spannungsspitzen an den Ecken. Diese Hypothese wird durch die Risslage an den Nuten bestärkt. Risse an Nuten treten stets an diesen Ecken auf (Abbildung 18). Einen Sonderfall bildet das Bohrloch mit Schraube. Die Schraube drückt das Bohrloch auseinander und induziert dadurch eine zusätzliche Spannung.

Bei der weiteren Anwendung dieser Methode wurde eine Rissinitiale aus beiden Gruppen verwendet: $5 \mathrm{~mm}$ Bohrloch ohne Schraube und eine einzelne diagonale Nut. 


\subsubsection{EINSEITIGE UND BEIDSEITIGE PRÜFUNG}

Es sollte geprüft werden, ob beidseitiges Einbringen von Rissinitialen einen Einfluss auf die Rissbildung und damit auf die Ansprache der Rissanfälligkeit hat. Beidseitig eingebrachte Rissinitialen würden eine effizientere Nutzung des Probenmaterials erlauben.

\section{ERGEBNISSE}

Tabelle 28: Kumulierte Risslänge [mm] direktbeschichteter Platten bei einseitigen und beidseitigen Rissinitialen

\begin{tabular}{|c|c|c|c|c|}
\hline \multirow[t]{3}{*}{ Platte } & \multicolumn{4}{|c|}{ Kumulierte Risslängen [mm] } \\
\hline & \multicolumn{2}{|c|}{ Prüfung an separaten Proben } & \multicolumn{2}{|c|}{ Prüfung an derselben Probe } \\
\hline & Vorderseite & Rückseite & Vorderseite & Rückseite \\
\hline A & 3 & 4 & 5 & 7 \\
\hline B & 5 & 6 & 11 & 13 \\
\hline $\mathrm{C}$ & 17 & 25 & 23 & 31 \\
\hline D & 45 & 51 & 47 & 62 \\
\hline $\mathrm{E}$ & 2 & 1 & 3 & 4 \\
\hline $\mathrm{F}$ & 3 & 5 & 4 & 7 \\
\hline G & 22 & 20 & 30 & 30 \\
\hline $\mathrm{H}$ & 24 & 24 & 34 & 37 \\
\hline$\Sigma$ & 121 & 136 & 157 & 190 \\
\hline$\sum$ & \multicolumn{2}{|c|}{257} & \multicolumn{2}{|c|}{347} \\
\hline
\end{tabular}

Platten mit nur einseitig eingebrachten Rissinitialen zeigen gegenüber Proben mit beidseitigen Rissinitialen stets weniger Risse (Tabelle 28). Die Rangfolge zur Rissanfälligkeit der Platten verändert sich nicht.

\section{DISKUSSION}

Die erhöhte Rissanfälligkeit bei beidseitig eingebrachten Rissinitialen ist nicht auf Ausreißer einzelner Platten zurückzuführen. Bei sämtlichen geprüften Platten ist die kumulierte Risslänge höher, wenn in beide Seiten Rissinitialen eingebracht werden. Unterschiedliche Prüfungsbedingungen lassen sich ausschließen. Alle Proben wurden gleichzeitig im selben Ofen belastet. Im Ofen waren die Proben zufällig auf mehrere Gestelle aufgeteilt.

Als mögliche Ursachen für höhere kumulierte Risslängen bei beidseitiger Prüfung einer Probe kommen das veränderte Schwindverhalten der Spanplatte sowie Spannungsspitzen durch Überlagerung der Spannungen der beidseitigen Rissinitialen infrage. An Rissinitialen ist ein schnellerer Feuchteaustausch der Spanplatte mit der trocknen Ofenluft möglich. Bei beidseitig eingebrachten Rissinitialen kann es zu einem schnelleren Schwund der Spanplatte kommen. Die Direktbeschichtung steht unter einer höheren Spannung und reißt daher stärker auf.

Bei der weiteren Anwendung der Methode wurden beidseitig Rissinitialen eingebracht. Die Rangfolge zur Rissanfälligkeit der Platten bleibt unverändert und das Prüfmaterial wird effizienter genutzt. 


\subsubsection{TeMPERATUR IM OfEn UND DAUER DER OfEnBELASTUNG}

\section{ERGEBNISSE}
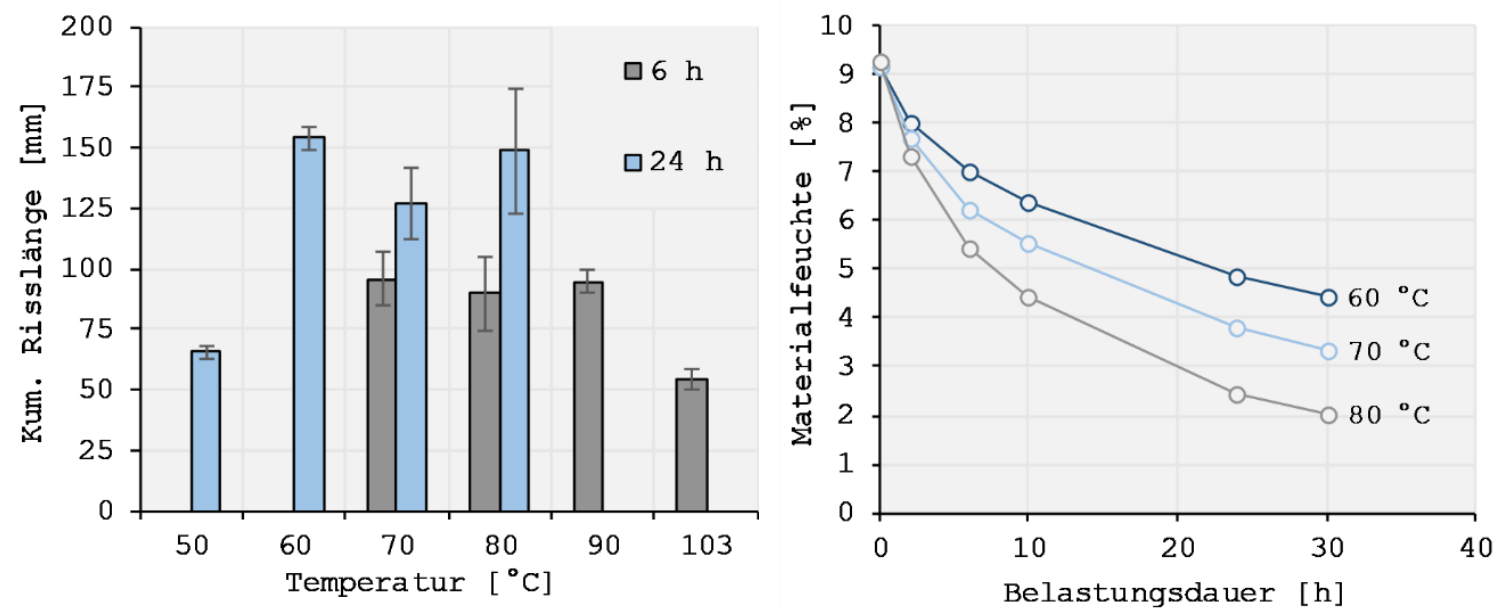

Abbildung 19: Gemittelte kumulierte Risslängen (links) und Materialfeuchten (rechts) von Probensätzen bei Variation von Temperatur und Dauer der Rissanfälligkeitsprüfung. Die kumulierten Risslängen bei $50{ }^{\circ} \mathrm{C}$ und $60{ }^{\circ} \mathrm{C}$ wurden nur nach 24 stunden und bei $90{ }^{\circ} \mathrm{C}$ und $103{ }^{\circ} \mathrm{C}$ nur nach 6 Stunden ermittelt.

Zwischen $60^{\circ} \mathrm{C}$ und $90^{\circ} \mathrm{C}$ sind die kumulierten Risslängen bei den jeweiligen Belastungsdauern relativ konstant (Abbildung 19). Bei 6 stündiger Belastung ist die kumulierte Risslänge geringer als nach 24 stündiger Belastung. Sowohl bei verringerter als auch bei erhöhter Temperatur $\left(50^{\circ} \mathrm{C} \mathrm{bzw} .103^{\circ} \mathrm{C}\right)$ sinkt die kumulierte Risslänge. Nach 24 stündiger Belastung ist bei keiner der geprüften Temperaturen die Ausgleichsfeuchte der Proben erreicht.

\section{DISKUSSION}

Eine Abhängigkeit der kumulierten Risslänge zur Materialfeuchte ist nicht erkennbar.

Bei der Rissanfälligkeitsprüfung treten hygrische Schwindungen und thermische Expansionen auf. Laut CARDARELLI (2018) liegt der thermische Ausdehnungskoeffizient von Melaminharz bei $22 * 10^{-6} / \mathrm{K}$. EYERER; ELSNER; HIRTH (2005) geben eine Spannweite von 40 bis $60 * 10^{-6} / \mathrm{K}$ an. Mit den Werten lässt sich die thermische Längenänderung bei $50^{\circ} \mathrm{C}$ berechnen: $0,07 \%$ bis $0,18 \%$. Die hygrische Schwindung des mit Harz imprägnierten Papiers (Werte stammen aus Kapitel 5.1.5.1) ist mit ca. 0,7\% um ein 4 bis 10-faches größer. Bei den geringeren Temperaturen im Ofen wird die Belastung von der hygrischen Schwindung dominiert. Bei $103^{\circ} \mathrm{C}$ steigt die thermische Längenänderung auf $0,18 \%$ bis $0,5 \%$. Die hygrische Schwindung ist mit $1,2 \%$ nur noch 2,4 bis 6 -fach so groß. Der Einfluss der thermischen Längenänderung steigt. Die Werte zur hygrischen und thermischen Längenänderung erklären jedoch nicht, warum es bei $60^{\circ} \mathrm{C}$ bis $80^{\circ} \mathrm{C}$ über 24 Stunden bzw. bei $70^{\circ} \mathrm{C}$ bis $90^{\circ} \mathrm{C}$ über 6 Stunden zu einer unveränderten Rissanfälligkeit und bei $103^{\circ} \mathrm{C}$ sogar zu einem Abfall der kumulierten Risslänge kommt. Möglich sind Spannungsverringerungen durch ein stärkeres Schwinden der Spanplatte oder eine höhere Elastizität der Direktbeschichtung bei höheren Temperaturen. Es handelt sich um einen komplexen Zusammenhang, der nicht geklärt werden konnte. 


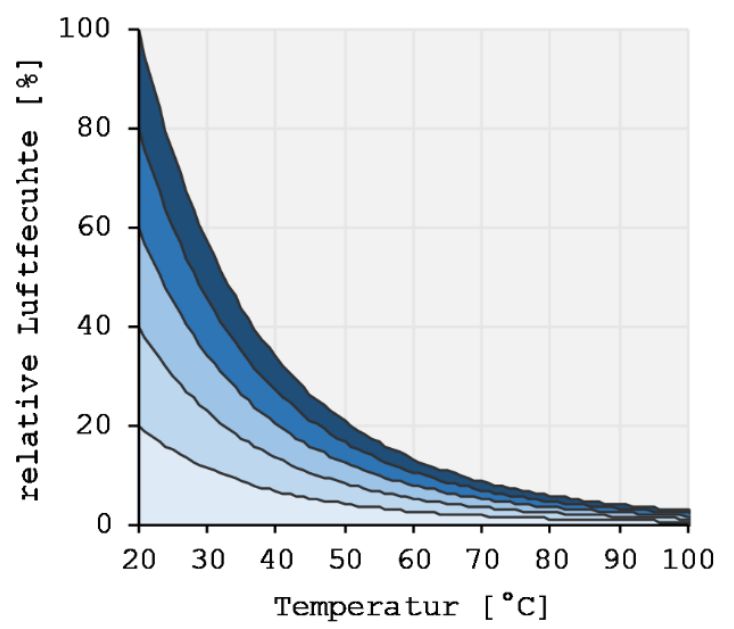

Abbildung 20: Verlauf der relativen Luftfeuchte verschiedener Ausgangsfeuchten bei Erhöhung der Temperatur

(Berechnet nach Formel 22)

Das Raumklima hat einen Einfluss auf die Ofenbelastung. Steigt die Raumfeuchte um den Ofen von $20 \%$ auf $60 \%$, erhöht sich die relative Luftfeuchte bei $50{ }^{\circ} \mathrm{C}$ im Ofen von $4,2 \%$ auf $12,5 \%$. Bei $70{ }^{\circ} \mathrm{C}$ würde sich die relative Luftfeuchte im Ofen nur von 1,7\% auf 5,2\% erhöhen. Bei höheren Ofentemperaturen nimmt die Spannweite der beiden relativen Luftfeuchte nur gering ab, der Einfluss der Ofentemperatur auf die relative Luftfeuchte sinkt. Bei der weiteren Anwendung der Methode wurde die Ofenbelastung bei $70^{\circ} \mathrm{C}$ durchgeführt. Diese Temperatur ermöglicht eine gute Differenzierung und die Luftfeuchte im Ofen ist kaum vom Raumklima beeinflusst.

Bei einer Prüfdauer von 6 Stunden kann die gesamte Prüfung innerhalb eines Arbeitstages erfolgen. Allerdings sind gegenüber der normgemäßen 24-stündigen Prüfung die kumulierten Risslängen geringer und die Differenzierbarkeit der Platten ist schwächer. Bei der weiteren Anwendung der Methode wurde daher eine 24-stündige Belastungsdauer gewählt.

\subsection{HYDROLYSEPRÜFUNGEN}

Es wurden Einflussgrößen auf die Hydrolyse von Direktbeschichtungen und auf die UV-Spektroskopie identifiziert und die Methode weiter optimiert.

\subsubsection{UV-SPEKTREN}<smiles>Nc1nc(N)nc(N)n1</smiles>

Melamin<smiles>Nc1nc(N)nc(O)n1</smiles>

Ammelin<smiles>Nc1nc(O)nc(O)n1</smiles>

Ammelid<smiles>Oc1nc(O)nc(O)n1</smiles>

Cyanursäure

Abbildung 21: Strukturformeln von Melamin und dessen Substitutionsprodukte

Melamin kann bei der Hydrolyse zu Ammelin dissoziieren (Abbildung 21), welches weiter zu Ammelid und Cyanursäure zerfallen kann (SCHRÖDER; FRANZ; HAGEN 1976). Es wurden die UV-Spektren von Melamin, dessen Substitutionsprodukte und Harnstoff (als möglicher Bestandteil bei Mischharzen) aufgenommen, um deren mögliche Beeinflussung der Hydrolyseprüfung zu bestimmen. 


\section{ERGEBNISSE}
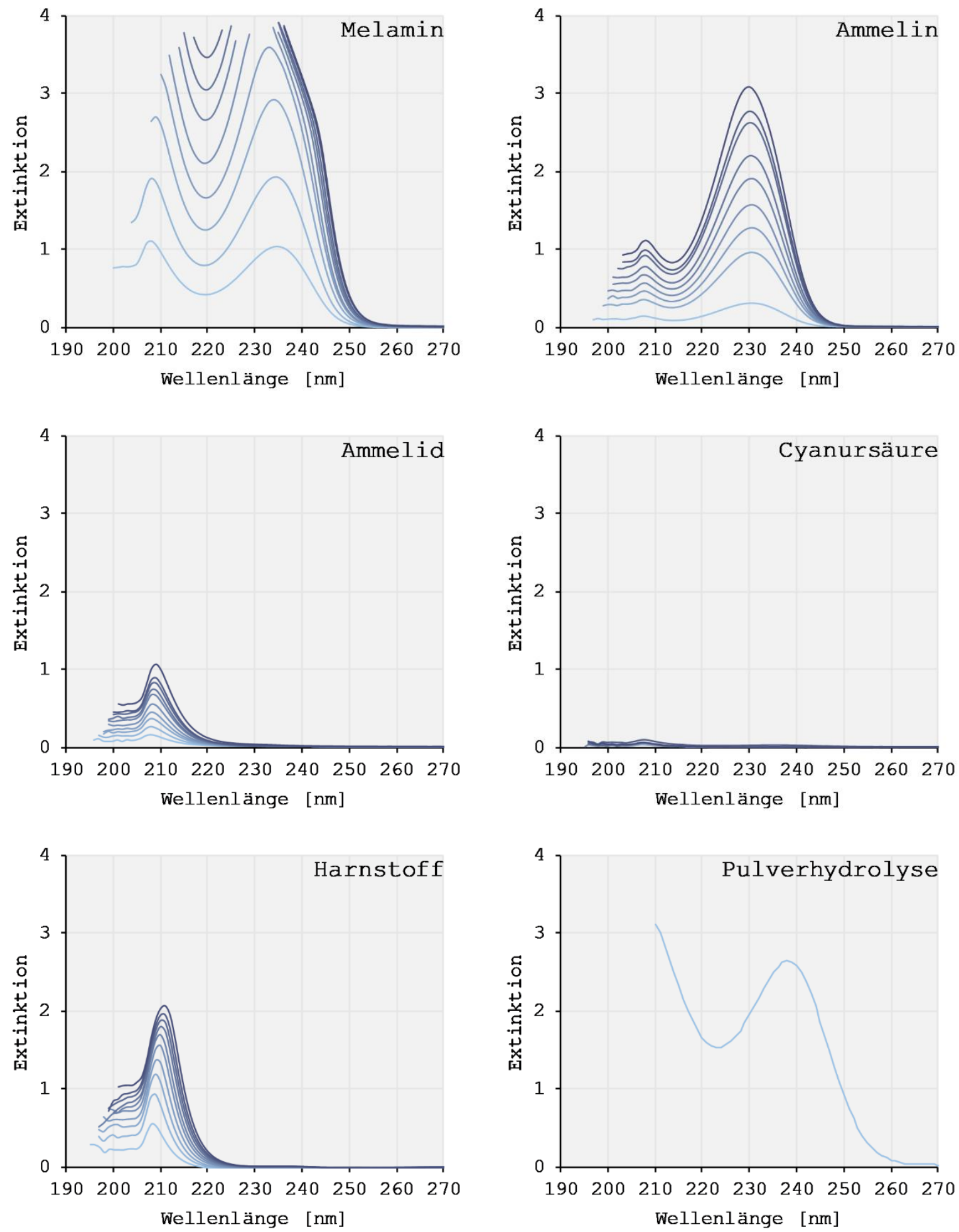

Abbildung 22: UV-Spektren von Melamin, Ammelin, Ammelid, Cyanursäure und Harnstoff sowie ein typisches Spektrum der Pulverhydrolyse einer reinen MF-Direktbeschichtung. Harnstoff: $20 \mathrm{~g} / \mathrm{l}$ bis $200 \mathrm{~g} / \mathrm{l}$, anderen Stoffe: $6 \mathrm{mg} / \mathrm{l}$ bis $54 \mathrm{mg} / \mathrm{I}$

Im UV-Spektrum von Melamin lassen sich Peaks bei $235 \mathrm{~nm}$ und $208 \mathrm{~nm}$ beobachten (Abbildung 22). Für Ammelin sind ebenfalls zwei Peaks zu beobachten; bei $230 \mathrm{~nm}$ und $208 \mathrm{~nm}$. Das Spektrum von Ammelid weist nur einen Peak bei 209 nm auf. Cyanursäure hat verschwindend geringe Extinktionen. Bei ca. $207 \mathrm{~nm}$ kann ein Peak beobachtet werden. Im UV-Spektrum der Pulverhydrolse einer 
MF-Direktbeschichtung ist ein Peak bei $237 \mathrm{~nm}$ zu beobachten. Unterhalb von $210 \mathrm{~nm}$ deutet sich ein Sekundärpeak an. Harnstoff zeigt je nach Stoffkonzentration einen Peak zwischen 208 nm und 212 nm. Mit abnehmender Konzentration sinkt der Peak und das Peakmaximum verschiebt sich zu geringeren Wellenlängen. Von $270 \mathrm{~nm}$ bis $900 \mathrm{~nm}$ ist keine der Substanzen UV/Vis-aktiv.

\section{DISSKUSSION}

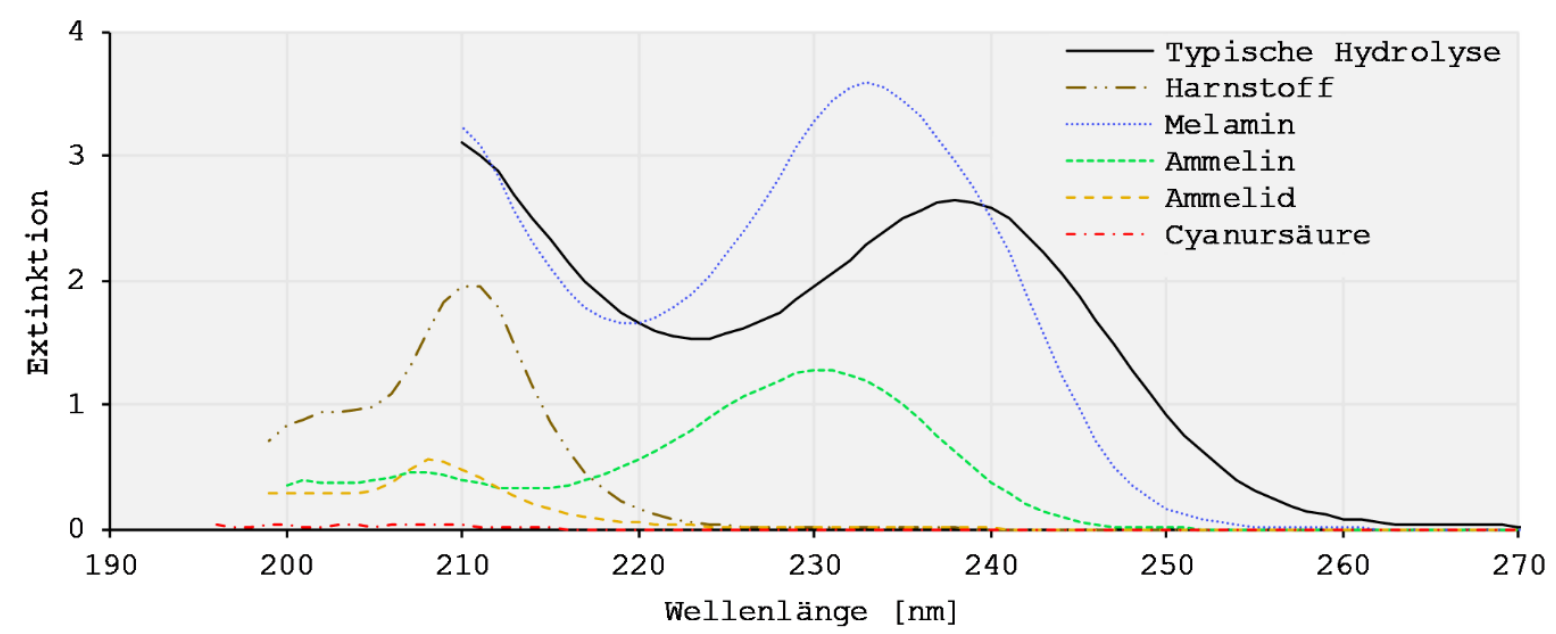

Abbildung 23: Extinktionen einer Pulverhydrolyse sowie von Harzbestandteilen.

Harnstoff: $200 \mathrm{~g} / \mathrm{l}$, andere Stoffe: $24 \mathrm{mg} / \mathrm{l}$

Mit fortschreitender Abnahme der Aminogruppen sinkt die Absorption und Peaks fallen weg (Abbildung 23). Wird Melamin zu Ammelin umgesetzt, entfällt der Peak bei $210 \mathrm{~nm}$ und die Extinktion über den gesamten Wellenlängenbereich nimmt ab. Zerfällt es weiter zu Ammelid, verschwindet der Peak bei $230 \mathrm{~nm}$. Bei Cyanursäure ist bei gleicher Konzentration fast keine Extinktion erkennbar.

Die Pulverhydrolyse einer MF-Direktbeschichtung zeigt ein ähnliches Spektrum wie Melamin. Abweichend ist jedoch, dass beim Pulverhydrolyse-Spektrum der primäre Peak bei höheren Wellenlängen liegt. Dies könnte durch einen bathochromen Effekt hervorgerufen werden. Durch die Methylolierung erhöht sich die Elektronendichte. Es wird weniger Energie - sprich kürzere Wellenlängen - zur Anregung benötigt. Die Pulverhydrolyse könnte auch statt reinem Melamin methyloliertes Melamin aus der Direktbeschichtung gelöst haben. Bei in Wasser gelöstem Melamin beobachten EBDon; HUNT; O'ROURKE (1987) einen Peak bei $205 \mathrm{~nm}$. Mit zunehmender Methylolierung verschiebt sich der Peak zu $215 \mathrm{~nm}$. Unterstellt man Melamin ein analoges Verhalten in Salzsäure sollte sich der $235 \mathrm{~nm}$ Peak von reinem Melamin bis zu ca. $245 \mathrm{~nm}$ bei hoher Methylolierung verschieben. Dieses Spektrum würde dem der Pulverhydrolyse gleichen. 


\subsubsection{ZeITLICHE VERÄNDERUNGEN DES SPEKTRUMS}

Nach dem Abfiltrieren des Pulvers bei einer Pulverhydrolyse könnten weiterhin Reaktionen in der Lösung stattfinden und das UV-Spektrum verändern. Ein strikt einzuhaltender zeitlicher Ablauf von Hydrolyse und UV-spektroskopischer Vermessung wäre notwendig. Um diese zeitliche Abhängigkeit zu überprüfen wurde das UV-Spektrum einer Pulverhydrolyse zu unterschiedlichen Zeiten aufgenommen.

\section{ERGEBNISSE}

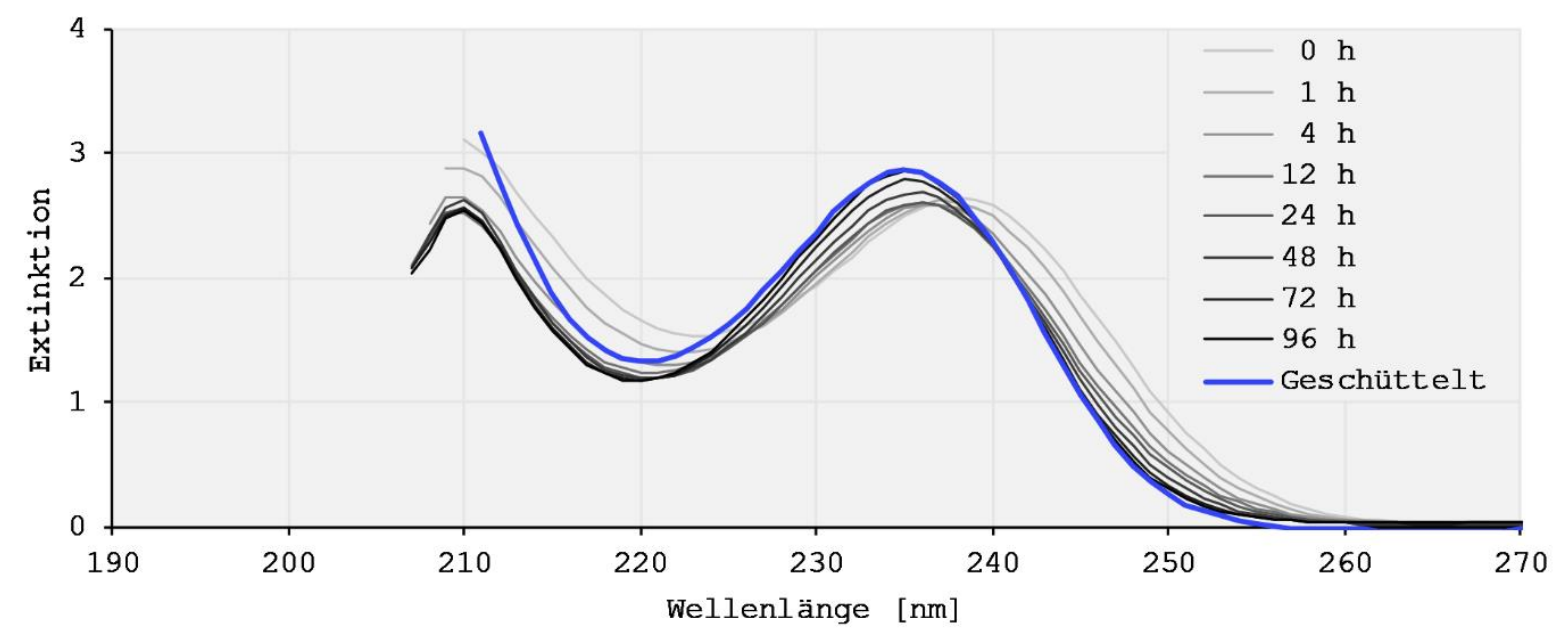

Abbildung 24: UV-Spektren derselben Hydrolyselösung direkt nach der Hydrolyse (0 h), nach mehrstündiger Lagerung ( 1 h bis 96 h) und geschüttelt nach 96-stündiger Lagerung

Nach Filtern des Pulvers aus der Hydrolyselösung einer MF-Direktbeschichtung können Änderungen im UV-Spektrum (Abbildung 24) beobachtet werden. In den ersten Stunden sinken die Extinktionen der Lösung unterhalb $225 \mathrm{~nm}$ und oberhalb $237 \mathrm{~nm}$. Zwischen $225 \mathrm{~nm}$ und $237 \mathrm{~nm}$ steigt die Extinktion nach 24 Stunden an. Durch Schütteln der Probe steigt die Extinktion unterhalb $230 \mathrm{~nm}$.

\section{DISKUSSION}

Aus Kapitel 4.2.1 sind die Spektren von Melamin und dessen Abbauprodukte bekannt. Ein Abbau reinen Melamins kann nicht zu den Veränderungen des Spektrums während der Lagerung führen. Im Laufe der Lagerung nähert sich das Spektrum der Pulverhydrolyse dem von reinem Melamin an, nicht dem eines Abbauprodukts. Wie bereits in Kapitel 4.2.1 diskutiert, könnte das Spektrum der Pulverhydrolyse durch methyloliertes Melamin geprägt sein. Im Laufe der Lagerung könnte das methylolierte Melamin abgebaut werden und dadurch eine Spektrenänderung zu reinem Melamin bewirken.

Das veränderte Spektrum nach dem Schütteln kann auf ein Konzentrationsgefälle zurückzuführen sein. Durch das Schütteln würde der Stoff verteilt und das Spektrum verändert sich. Um welchen Stoff es sich dabei handelt, wurde nicht untersucht.

Ein strikt einzuhaltender zeitlicher Ablauf nach dem Filtrieren ist nicht notwendig. Proben können mindestens für 96 Stunden eingelagert werden. 


\subsubsection{HYDROLYSIERTES MELAMIN}

Die Angabe der Extinktion als Ergebnis der Hydrolyselösung hat Nachteile. Das Lambert-Beer'sche Gesetz (Zusammenhang zwischen Extinktion und Stoffkonzentration) gilt nur im linearen Bereich. Ist die Extinktion zu hoch, geht die Linearität verloren. Die Lösung muss verdünnt und erneut vermessen werden. Bei einer Angabe der Stoffkonzentration lässt sich eine solche Verdünnung einbeziehen. Es wurde daher das Spektrometer kalibriert, um die Stoffkonzentration als Messergebnis angeben zu können.

\section{ERGEBNIS}

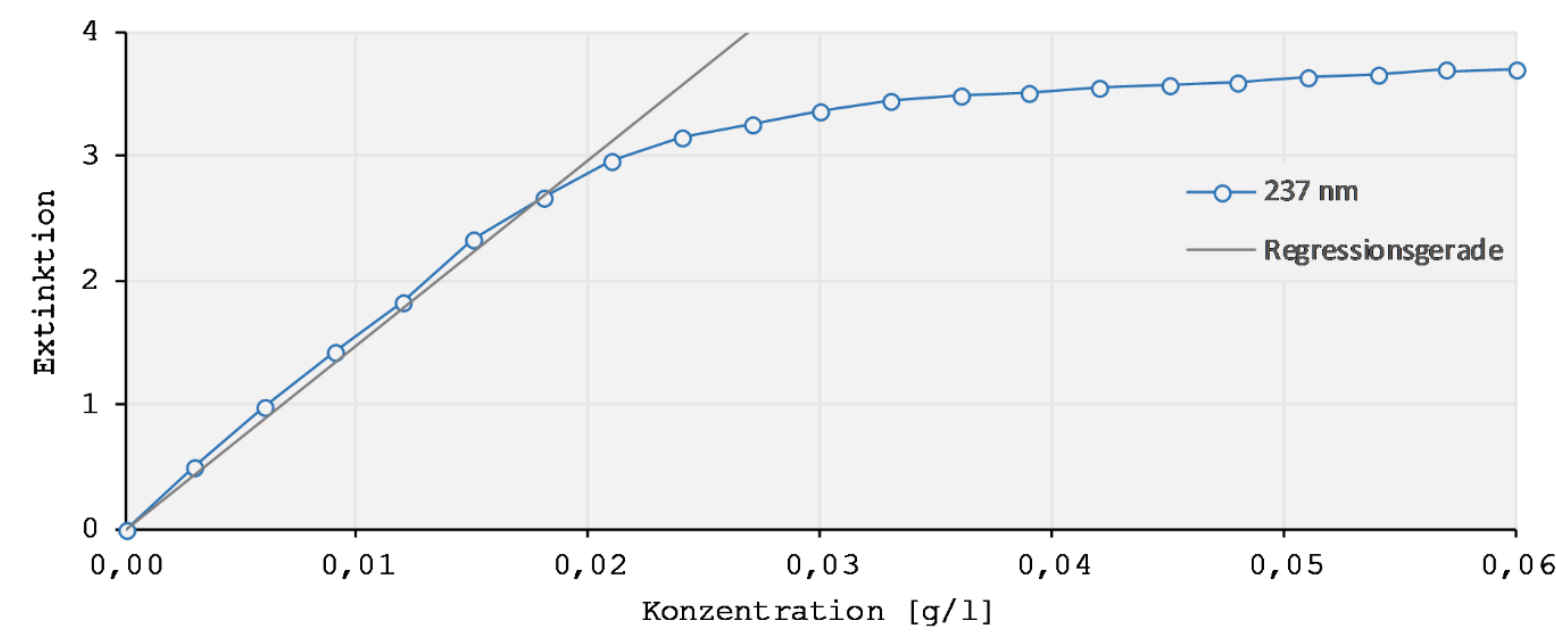

Abbildung 25: Extinktionen bei $237 \mathrm{~nm}$ von Melamin unterschiedlicher Konzentrationen gelöst in Salzsäure (15\%-ig)

Zur Quantifizierung einer Melaminkonzentration in einer Lösung wird von SCHRÖDER; FRANZ; HAGEN (1976) auf die Extinktion bei $237 \mathrm{~nm}$ verwiesen. Melamin weist bei dieser Wellenlänge einen ausgeprägten, linearen Zusammenhang zwischen Extinktion und Konzentration auf (Abbildung 25). Dieser Zusammenhang ist beim verwendeten UV-Vis-Spektrometer für eine Extinktion unterhalb von 3 gegeben. Aus der Extinktion und der Regressionsgeraden ergibt sich für Formel 14 zur Quantifizierung des Melamins ein Extinktionskoeffizient von $k_{237}=152,7 \mathrm{l} / \mathrm{g}$.

\section{DISKUSSION}

Im Weiteren wird statt der Extinktion die errechnete Stoffkonzentration des hydrolysierten Melamins angegeben. Wie in Kapitel 4.2.1 aufgeführt, handelt es sich beim hydrolysierten Stoff nicht um reines Melamin. Entsprechend ist der errechnete Wert nicht als Melaminkonzentration, sondern als melaminäquivalente Konzentration zu verstehen. 


\subsubsection{UMGEBUNGSTEMPERATUR, HYDROLYSEDAUER UND SÄUREKONZENTRATION}

Die Hydrolyseprüfung dauerte ursprünglich 2 Stunden. Durch Variation verschiedener Stellgrößen sollte geprüft werden, ob die Methode beschleunigt werden kann ohne Genauigkeit einzubüßen und ob eine längere Prüfung Vorteile bringt.

\section{ERGEBNISSE}
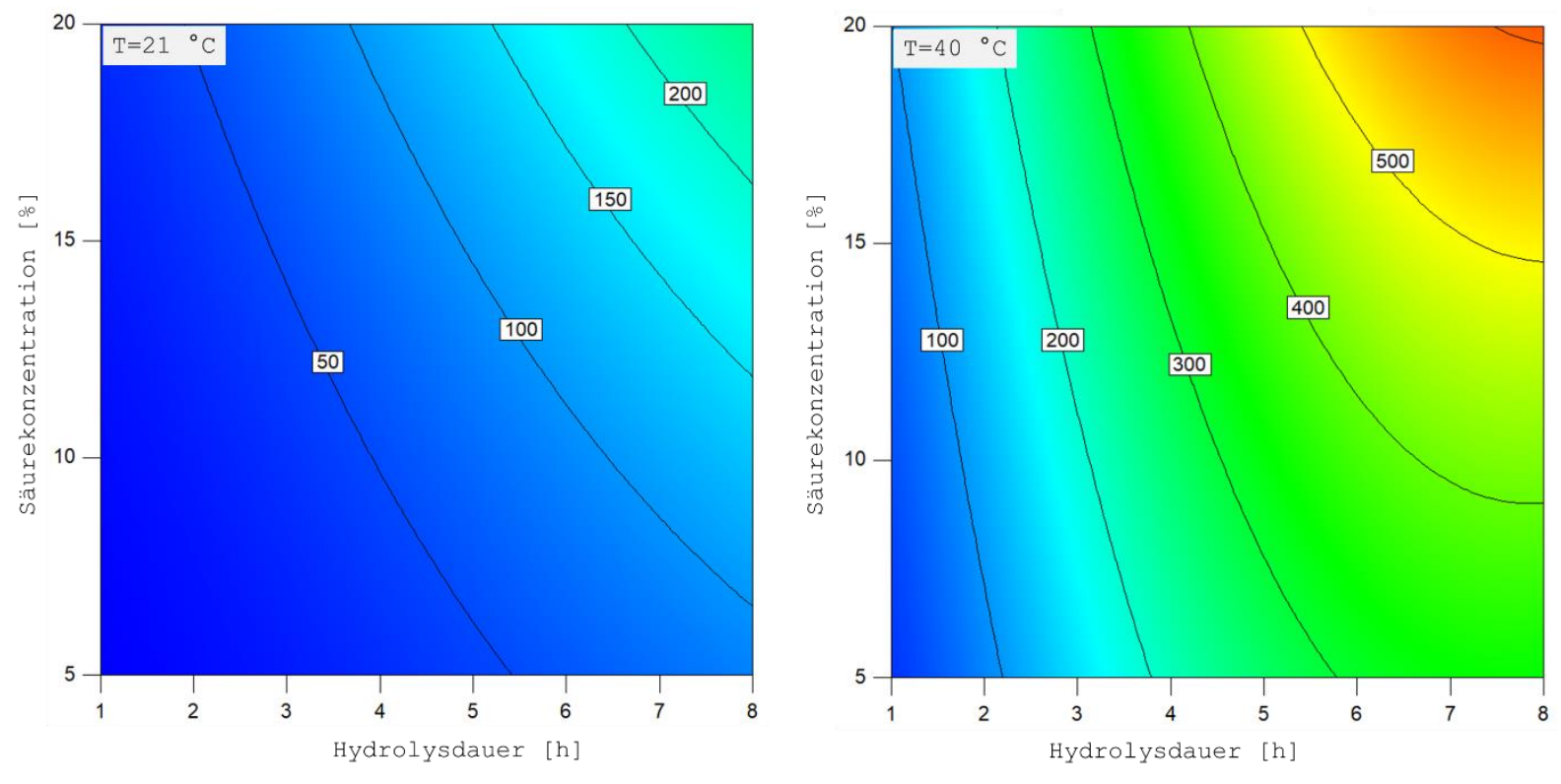

Abbildung 26: Hydrolysierte Melaminmasse [pg] für Hydrolysen durchgeführt bei einer Umgebungstemperatur von $21{ }^{\circ} \mathrm{C}$ (links) und $40{ }^{\circ} \mathrm{C}$ (rechts) mit Säurekonzentrationen von 5\% bis 20\% und Hydrolysedauern von 1 h bis $8 \mathrm{~h}$

Eine Erhöhung der Säurekonzentration, Hydrolysedauer oder Umgebungstemperatur hat einen Anstieg des hydrolisierten Melamins zur Folge (Abbildung 26).

\section{DISKUSSION}

Die Säurehydrolyse der Direktbeschichtung folgt offensichtlich der Van't-Hoff'schen Regel: eine Erhöhung der Umgebungstemperatur um 10 Kelvin führt zu einer Verdoppelung bis Vervierfachung der Reaktionsgeschwindigkeit. Die Temperaturabhängigkeit der Reaktion verbietet es, die Hydrolyse in nicht temperierten Räumen durchzuführen. Bereits eine Temperaturänderung von $1{ }^{\circ} \mathrm{C}$ kann den Messwert um bis zu 15\% verändern. Da Laboratorien üblicherweise keine genaue Temperierung besitzen, sollte die Reaktion in einem Wärmeschrank oder Wasserbad oberhalb der maximalen erwartbaren Raumtemperatur erfolgen. Zudem läuft die Reaktion schneller ab, man erhält schneller ein Messergebnis. Durch die Wahl einer hohen Säurekonzentration kann die Hydrolysedauer weiter verkürzt werden. Es zeigt sich keine Abnahme der Genauigkeit. 


\subsubsection{REINIGUNGSMITTEL}

Verunreinigung der zu hydrolysierenden Oberfläche könnten einen Einfluss auf das Messergebnis haben. Mit Reinigungsmitteln können zwar Verunreinigung entfernt werden. Allerdings könnten die Reinigungsmittel ebenfalls die Hydrolyse beeinflussen. Es sollte daher geprüft werden, ob eine Reinigung der Oberfläche das Messergebnis beeinträchtigt.

\section{ERGEBNISSE}

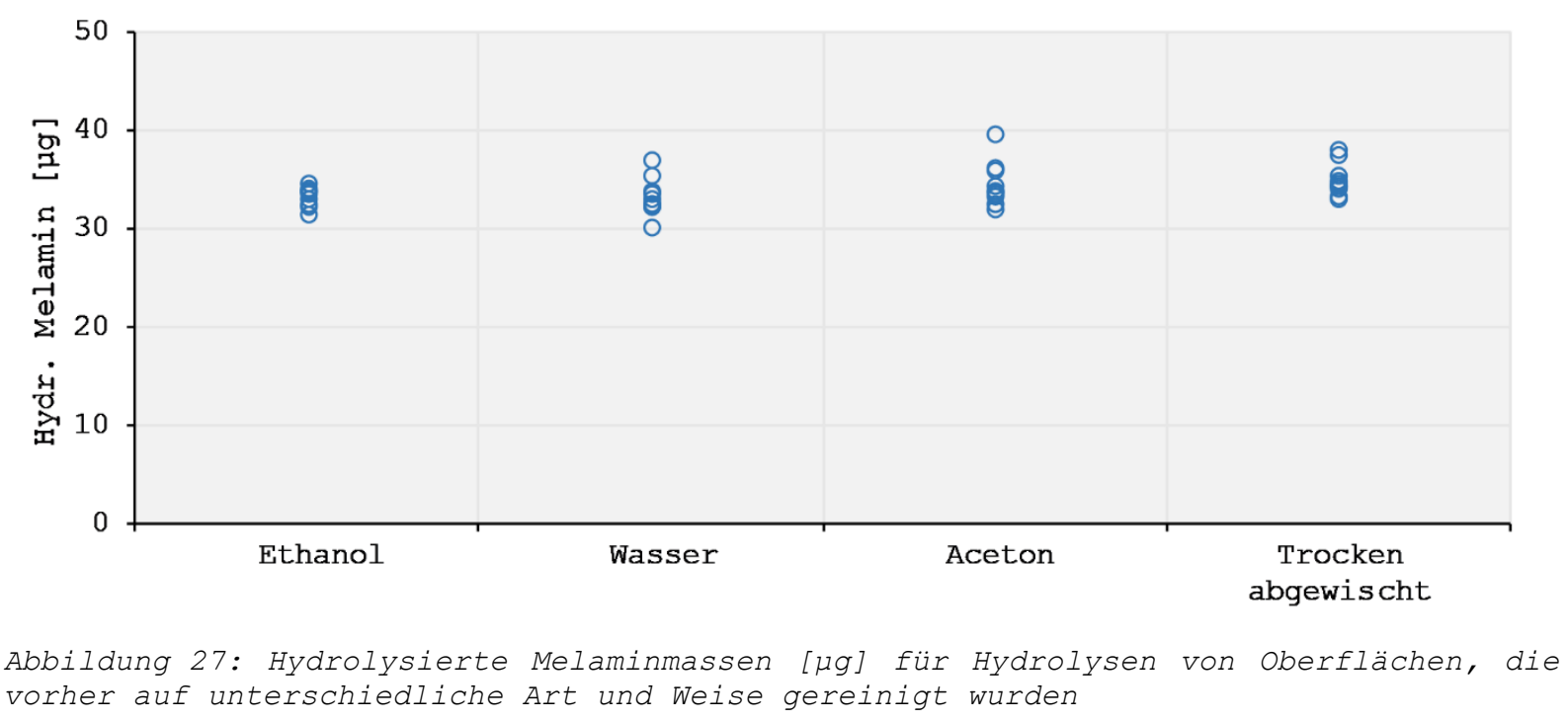

Die Messwerte aller Reinigungsmittel sind gleich. Das Messergebnis ist nicht abhängig vom Reinigungsmittel (Abbildung 27).

\section{DISKUSSION}

Oberflächenverschmutzung können einen Einfluss auf die Messwerte haben. Es sollte daher stets eine Oberflächenreinigung vor der Hydrolyse erfolgen. Die geprüften Reinigungsmittel verändern nicht die Messung. Sowohl Ethanol, Aceton als auch Wasser können daher verwendet werden.

Bei der Anwendung der Methode waren sämtliche Proben dieser Arbeit augenscheinlich sauber, sie wurden jedoch präventiv mit Aceton gereinigt.

\subsubsection{VERGLEICH DES HYDROLYSEVERFAHRENS ZUM KITON-TEST}

Der Kiton-Test ist die verbreitetste Prüfung des Aushärtungsgrades von Direktbeschichtungen. KitonTest und Hydrolyseprüfung wurden verglichen und geprüft, welche Methode den Aushärtungsgrad besser differenziert.

Es wurden 9 Platten aus der Versuchsreihe zu Aushärtungsbedingungen (Kapitel 5.1) verwendet. Sie unterscheiden sich hinsichtlich des Aushärtungsgrades, der über die Pressdauer und -temperatur eingestellt wurde. 


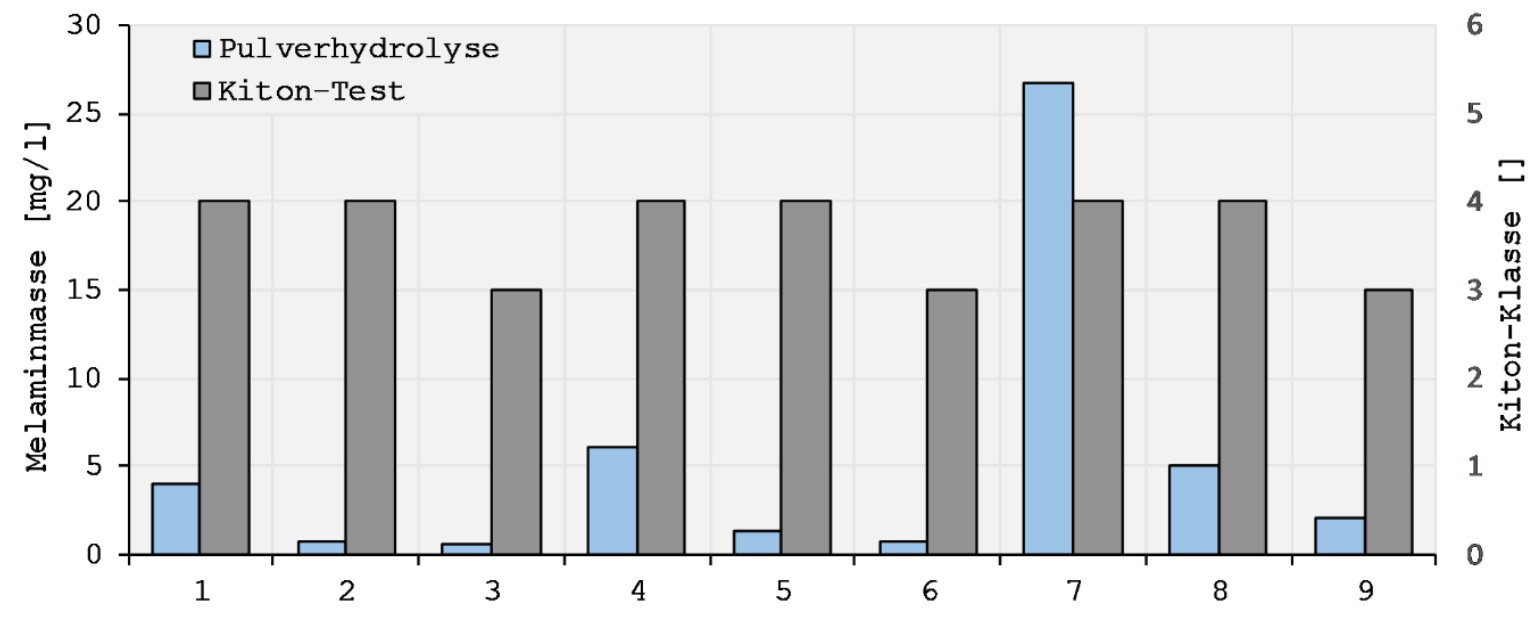

Abbildung 28: Aushärtungsgrade nach der Pulverhydrolyse und nach dem Kiton-Test

Der Kiton-Test zeigte bei den Platten lediglich 2 unterschiedliche Klassen des Aushärtungsgrades (Abbildung 28). Die Spannweite bei der Hydrolyseprüfung ist größer.

\section{DISKUSSION}

Folgende Vorteile bietet die Pulverhydrolyse gegenüber einem Kiton-Test:

1. Mittels der Pulverhydrolyse lässt sich der Aushärtungsgrad der Platten stärker differenzieren.

2. Bei der Hydrolyse wird ein objektiver Messwert ausgegeben. Beim Kiton-Test muss der Verfärbung durch den Prüfer subjektiv eine Klasse zugewiesen werden.

3. Bei der Pulverhydrolyse ist die Prüftemperatur festgelegt. Der Kiton-Test basiert auf demselben Wirkprinzip, eine Säurereaktion mit der Oberfläche. Auch beim Kiton-Test ist ein Einfluss der Umgebungstemperatur auf die Reaktionsgeschwindigkeit und damit das Ergebnis zu erwarten. Beim Kiton-Test nach AS/NZS 4266.21 (2004) wird diese Einflussgröße allerdings vernachlässigt.

4. Die Pulverhydrolyse ist mit 5 Minuten deutlich schneller als der zweistündige Kiton-Test.

5. Dunkle Oberflächen lassen sich mit dem Kiton-Test nicht bewerten.

6. Beim Kiton-Test tritt die Färbelösung in oberflächennahe Fasern ein und färbt diese. Letztlich bewertet der Test daher, wie lange die Harzschicht über den Papierfasern der Säurelösung widersteht. Bereits eine geringfügig höhere Harzbeaufschlagung kann zu veränderten Resultaten führen. Vergleichende Aussagen von Kiton-Tests sind daher nur an Direktbeschichtungen desgleichen Imprägnats gültig. (LUKOWSKY 2015; BöTTCHER; SAGROSKE 1985).

Ein Nachteil der Pulverhydrolyse ist der Arbeitsaufwand. Beim Kiton-Test muss nur Säure auf die Oberfläche gegeben werden. Zwei Stunden später wird die Verfärbung bewertet. Während den zwei Stunden können andere Tätigkeiten durchgeführt werden. Bei der Pulverhydrolyse muss Pulver abgeschliffen und eingewogen, die Hydrolyse durchgeführt und die Lösung spektroskopisch vermessen werden. Die Arbeitszeit bei der Pulverhydrolyse ist daher länger als beim Kiton-Test. 


\subsection{Model ZUR BereChNUng MeChANISCHER KenNWERTE DeR DIREKTBESCHICHTUNG}

Um den Einfluss der Eingabewerte des Models auf die Zugfestigkeit und das Elastizitätsmodul zu bestimmen, wurde eine Sensitivitätsanalyse durchgeführt.

\section{ERGEBNISSE}
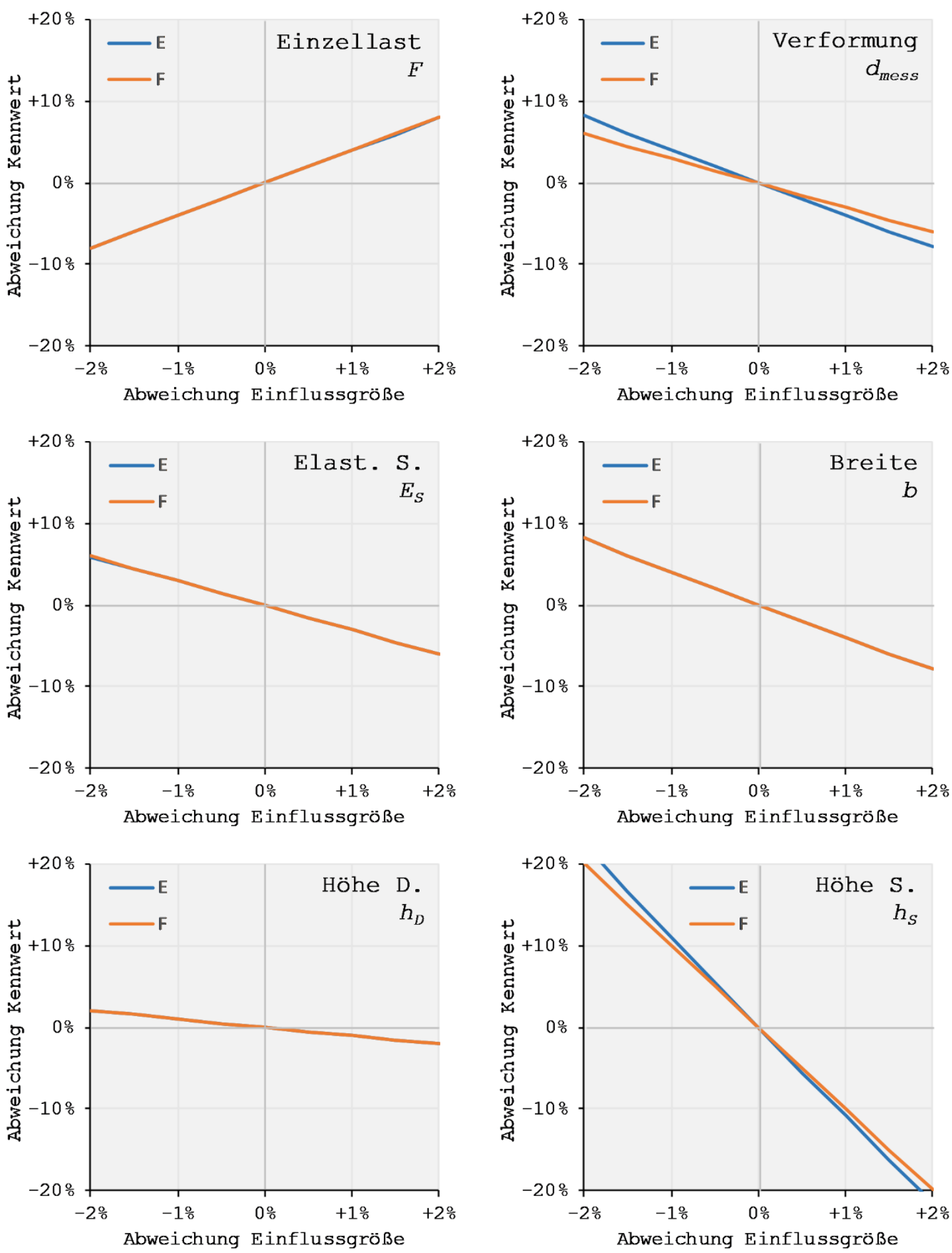

Abbildung 29: Sensitivitätsanalyse zum Model

E: Elastizitätsmodul der Direktbeschichtung, F: Festigkeit der Direktbeschichtung S. Spanplatte, D: Direktbeschcihtung, Elast. S.: Elastizitätsmodul der Spanplatte 
Sämtliche Einflussgrößen zeigen einen linearen Zusammenhang der untersuchten Variation zur Einflussgröße (Abbildung 29). Die als Einzellast $F$ und Verformung $d_{\text {mess }}$ bezeichneten Werte entsprechen den Werten des Messpunkts im linearelastischen Bereich.

\section{DISKUSSION}

Einzellast, Verformung und Elastizitätsmodul der Spanplatte werden während der Biegeprüfung von der Prüfmaschine aufgenommen und dokumentiert. Realistische Schwankungen dieser Werte $(0,1 \%)$ haben kaum einen Einfluss auf das Model. Bei der Probenbreite sind Abweichungen von 0,05 $\mathrm{mm}$ bei $50 \mathrm{~mm}$ Probendicke realistisch. Ein solche Abweichung verändert die mechanischen Kennwerte nur um $0,4 \%$. Auch die Höhe der Direktbeschichtung hat einen vergleichsweisen geringen Einfluss. Selbst eine Überschätzung der Schichtdicke von $2 \%$ führt nur zu einer 2\%-igen Abweichung beider mechanischer Kennwerte. Bei einer Abweichung der Spanplattenhöhe von 0,1 mm bei einer $16 \mathrm{~mm}$ Spanplatte verändern sich die mechanischen Kennwerte der Direktbeschichtung um lediglich $0,7 \%$.

Realistische Schwankungen der Kennwerte haben nur einen geringen Einfluss auf das Model. 


\section{ERGEBNISSE \& DISKUSSION ZU EINFLUSSGRÖßEN}

\subsection{EINFLUSSGRÖßE: AUSHÄRTUNGSBEDINGUNGEN}

Es wurde untersucht, wie Aushärtungsbedingungen die Rissanfälligkeit beeinflussen. Dazu wurden direktbeschichtete Platten mit verschiedenen Aushärtungsgraden produziert. Der Aushärtungsgrad wurde über die Pressdauer und -temperatur eingestellt. Es wurde sowohl mit kaltem als auch mit vorgeheiztem Pressblech beschichtet. Zusätzlich wurden freie Filme produziert und Spanplatten sowie Imprägnierpapiere analog eines Beschichtungsprozesses heißverpresst.

\subsubsection{RISSANFÄLLIGKEIT}

Es wurde geprüft, wie ausgeprägt die Rissanfälligkeit der produzierten Platten ist. Dazu wurden Prüfkörper $(n=3)$ mit beidseitig eingebrachten Rissinitialen über 24 Stunden bei $70^{\circ} \mathrm{C}$ belastet und deren kumulierte Risslänge als Maß für die Rissanfälligkeit der Platte bestimmt.

\section{ERGEBNISSE}

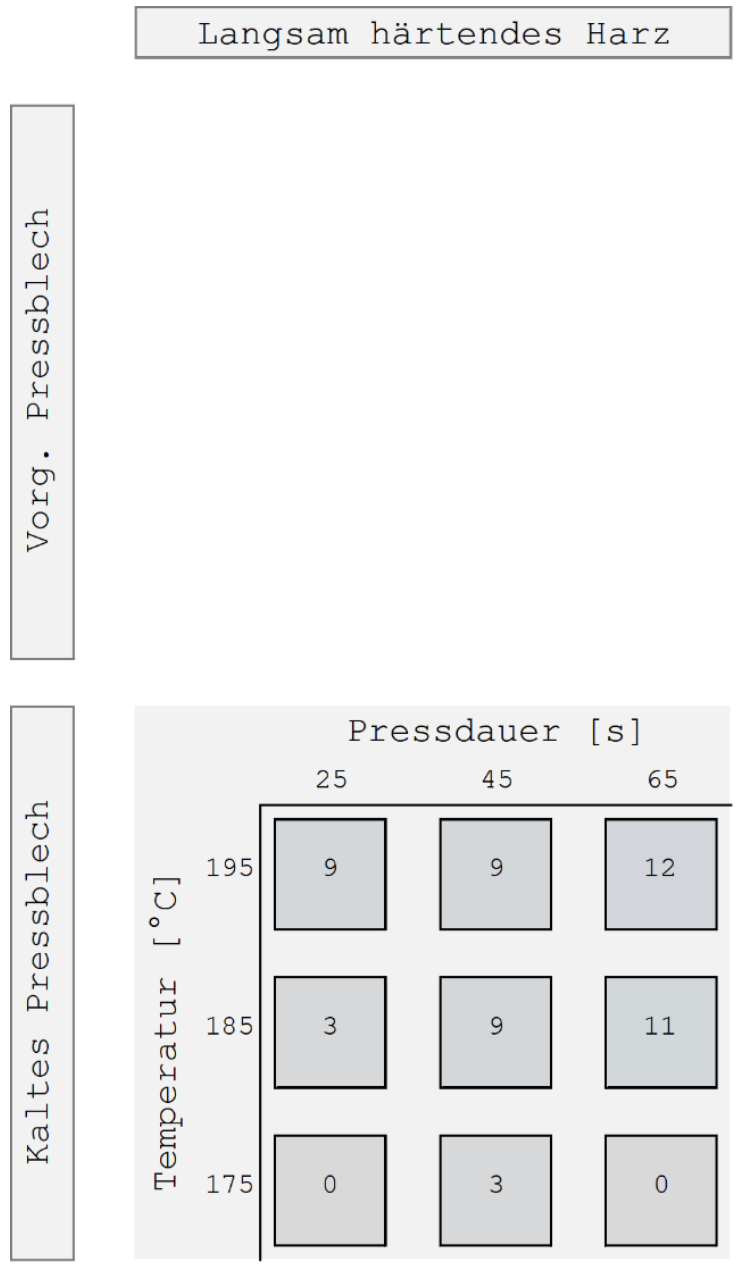

Abbildung 30: Kumulierte Risslängen unterschiedlicher Aushärtungsgrade.

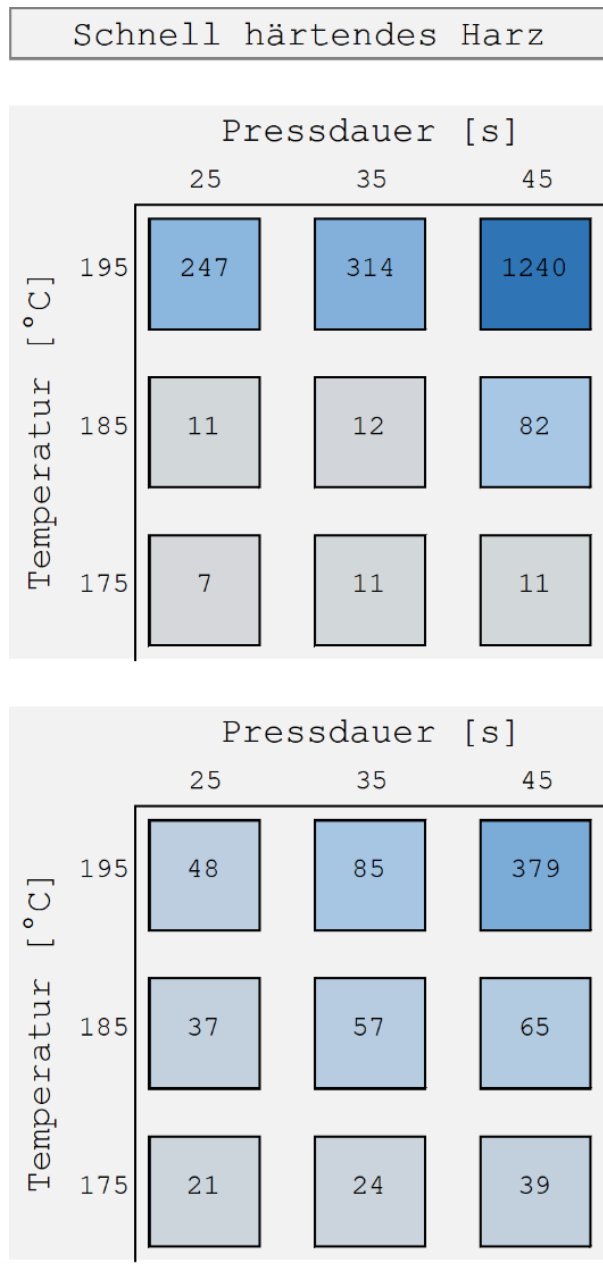

[mm ] direktbeschichteter

Platten

Die kumulierten Risslängen sind abhängig von den Aushärtungsbedingungen (Abbildung 30). Je höher der Energieeintrag beim Verpressen (längere Pressdauer bzw. höhere Presstemperatur), desto höher ist die Rissanfälligkeit der Platte. Die ANOVA-Modelle (siehe Anhang) bestätigen einen signifikanten Zusammenhang. Dieser Trend ist beim langsam härtenden Harz schwach ausgeprägt; selbst bei einer 
starken Überhärtung $\left(195^{\circ} \mathrm{C}, 65 \mathrm{~s}\right)$ entstehen lediglich $12 \mathrm{~mm}$ kumulierte Risslänge. Beim schnell härtenden Harz wurden bei demselben Pressverfahren dagegen bis zu 379 mm Risse gemessen.

Die kumulierten Risslängen bei mit vorgeheiztem Pressblech beschichteten Platten sind teilweise höher und teilweise niedriger als ihre mit kaltem Pressblech produzierten Gegenstücke. Unterhärtete Platten sind weniger gerissen, überhärtete stärker.

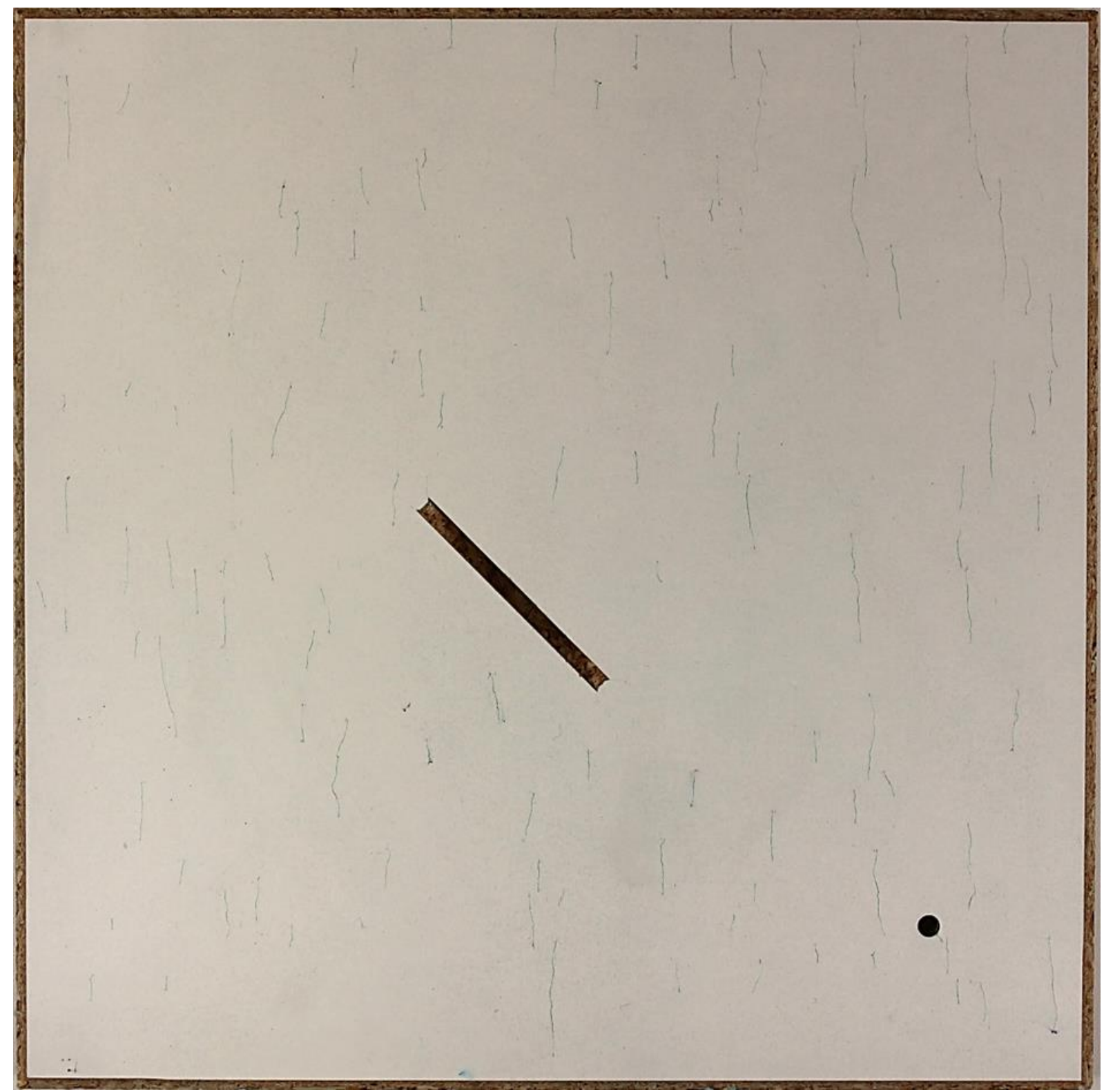

Abbildung 31: Stark überhärtete Probe $(25 \mathrm{~cm} \times 25 \mathrm{~cm})$ mit schnell härtendem Harz nach der Rissanfälligkeitsprüfung.

Risse sind zur Kontrastierung angefärbt.

Das Rissbild (Abbildung 31) einer stark überhärteten Probe ist geprägt durch viele über die Fläche verteilten Risse. Bei unterhärteten bis leicht überhärteten Proben sind nur Risse an Nuten und Bohrlöchern entstanden. 


\section{DISKUSSION}

Ein Zusammenhang zwischen erhöhter Rissanfälligkeit und Überhärtung gilt in der Branche als gesichert. Veröffentlichungen dazu konnten jedoch nicht gefunden werden. Die kumulierten Risslängen bestätigen diese Abhängigkeit. Zudem besteht eine Abhängigkeit der Rissanfälligkeit vom Harztyp. Die rissanfälligste Platte des langsam härtenden Harzes reißt weniger als die rissunempfindlichste des schnell härtenden Harzes. Eine erhöhte Rissanfälligkeit auf Grund eines nicht vergleichbaren, höheren Aushärtungsgrads lässt sich jedoch ausschließen. Die Aushärtungsgrade beider Harze bewegen sich in einem vergleichbaren Rahmen (Kapitel 5.1.8).

Optimal gehärtete und unterhärtete Platten reißen weniger, wenn sie mit vorgeheiztem Pressblech beschichtet wurden. Zu erwarten war eine höhere kumulierte Risslänge aller mit vorgeheizten Pressblech produzierten Platten. Die mit vorgeheiztem Pressblech produzierten Platten wurden zu einem späteren Zeitpunkt in einem anderen Ofen geprüft. Dies legt den Verdacht nahe, dass die Belastungen der Probengruppen in den Öfen nicht gleich waren. Eine geringere Temperatur lässt sich als Ursache ausschließen. Laut den Untersuchungen zur Rissanfälligkeitsmethode führt selbst eine Temperaturschwankung von $10^{\circ} \mathrm{C}$ nicht zu einer Änderung der kumulativen Risslänge. Möglicherweise war der Luftstrom im zweiten Ofen schwächer, die Proben wurden sanfter belastet. In dem Fall würden die Rissanfälligkeiten sämtlicher mit vorgeheiztem Pressblech beschichteten Platten unterschätzt.

Bei stark überhärteten Platten entstehen viele Risse bei der Prüfung abseits der Rissinitialen. Nut und Bohrloch sind bei diesen Platten nicht nötig, um die Rissanfälligkeit dieser Platte zu beurteilen. Im Gegensatz dazu reißen unterhärtete und leicht überhärtete Proben nur an Nut oder Bohrloch. Bei diesen Platten sind die Rissinitialen essentiell zur Differenzierung der Rissanfälligkeit. 


\subsubsection{FESTIGKEITEN UND ELASTIZITÄTSMODULN}

Bei einer Überhärtung könnten sich mechanische Eigenschaften verschlechtern und die erhöhte Rissanfälligkeit hervorrufen. Um einen solchen Einfluss zu prüfen, wurde von den direktbeschichteten Platten Festigkeiten und Elastizitätsmoduln aufgenommen. Um den Ursprung der dabei aufgedeckten Festigkeitsabnahme nachzuvollziehen, wurden nachfolgend auch die mechanischen Kennwerte der einzelnen Komponenten (Direktbeschichtungen anhand freier Filme, unbeschichtete Spanplatten sowie nicht imprägniertes Papier) geprüft.

\subsubsection{DIREKTBESCHICHTETE PLATTEN}

Um einen Einfluss des Aushärtungsgrades auf die mechanischen Kennwerte der direktbeschichteten Platten nachzuweisen wurden Proben $(n=6)$ im 3-Punkt-Biegeversuch geprüft. Die Festigkeit der Direktbeschichtung auf der zugbelasteten Seite (auf der der Bruch auftritt) wurde berechnet.

\section{ERGEBNISSE}
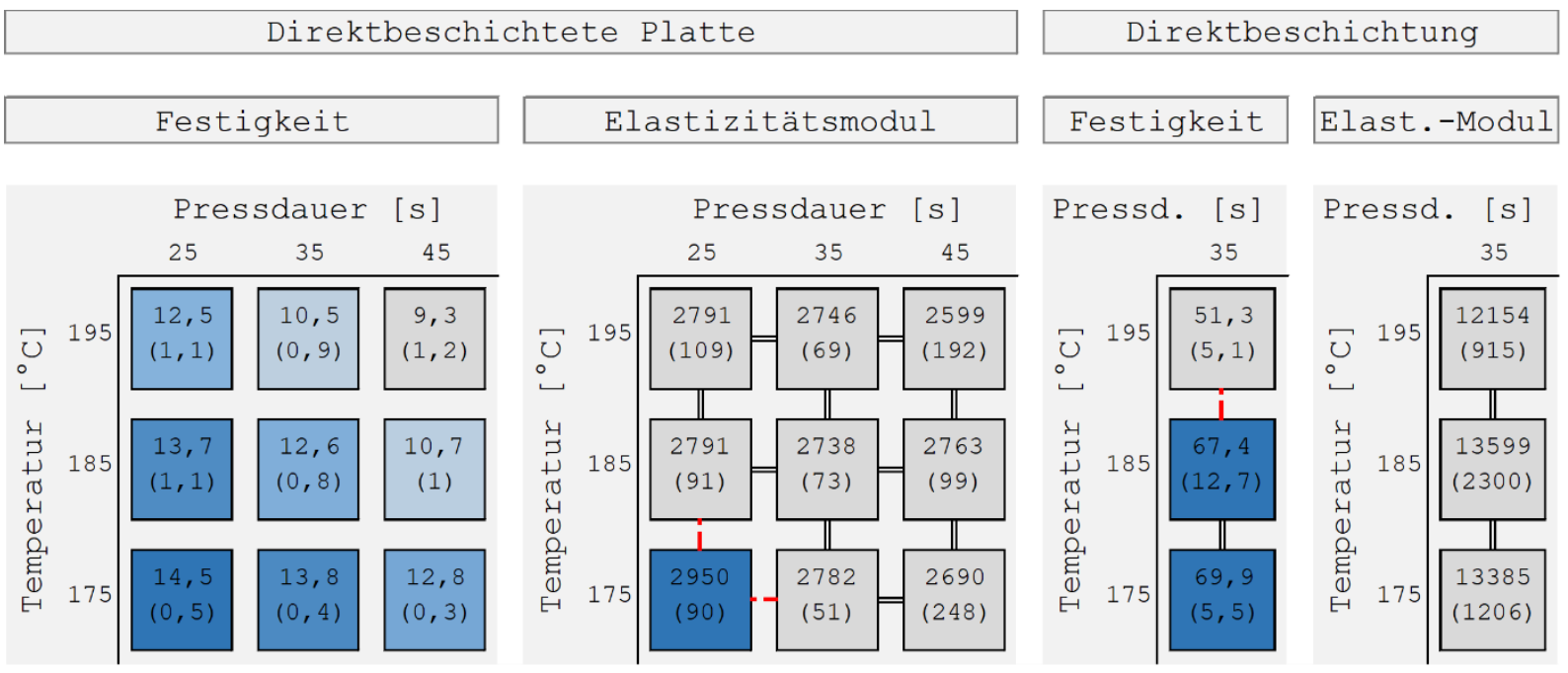

Abbildung 32: Gemessene Biegefestigkeiten [N/ $\mathrm{mm}^{2}$ ] und Elastizitätsmoduln [N/mm²] Von direktbeschichteten Platten ( $n=6)$ sowie berechnete Zugfestigkeiten und Elastizitätsmoduln von Direktbeschichtungen unterschiedlicher Aushärtungsgrade.

Doppellinie: kein signifikanter Unterschied, gestrichelte Linie: signifikanter Unterschied, in Klammern: Standardabweichung

Mit zunehmender Pressdauer und -temperatur sinkt die Biegefestigkeit der direktbeschichteten Platten (Abbildung 32). Das ANOVA-Model (siehe Anhang) bestätigt einen hoch-signifikanten Einfluss beider Parameter auf die Biegefestigkeit. Die Elastizitätsmoduln hingegen zeigen keine signifikanten Unterschiede mit Ausnahme der stark unterhärteten Platte. Diese ist steifer.

Die berechneten Elastizitätsmoduln der Direktbeschichtung sind nicht signifikant unterschiedlich. Die berechneten Zugfestigkeiten der $195^{\circ} \mathrm{C}$-Platten sind signifikant geringer (95\%-Niveau). 


\section{DISKUSSION}

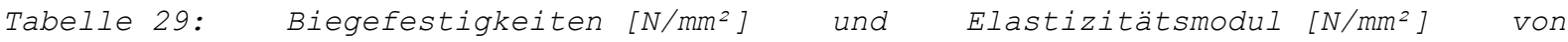
direktbeschichteten Spanplatten.

\begin{tabular}{l|l|l} 
& $\begin{array}{l}\text { Festigkeit } \\
{\left[\mathrm{N} / \mathrm{mm}^{2}\right]}\end{array}$ & $\begin{array}{l}\text { Elastizitätsmodul } \\
{\left[\mathrm{N} / \mathrm{mm}^{2}\right]}\end{array}$ \\
\hline Direktbeschichtete Platte & $9,3-14,5$ & $2600-2950$ \\
- Messwert & $14,5-16,6$ & $1900-2380$ \\
- NemLI; ÇOLAKOG ${ }^{2}$ LU (2005) & 9 & 2150 \\
Unbeschichtete Spanplatte & 1350
\end{tabular}

Die von NEMLI; ÇOLAKOG ¿̌ (2005) bestimmten mechanischen Kennwerte direktbeschichteter Platten (Tabelle 29) weichen von den Werten des hier geprüften Untersuchungsmaterials ab. Aufgrund der analog abweichenden Kennwerte bei den unbeschichteten Spanplatten ist dies plausibel.

Die höhere Steifigkeit der unterhärteten Platte kann durch eine höhere Steifigkeit der Direktbeschichtung hervorgerufen werden. Wie in Kapitel 5.1.2.3 gezeigt wird, haben unterhärtete freie Filme eine höhere Steifigkeit.

Mittels des Rechenmodels lassen sich Zugfestigkeiten der Direktbeschichtung bestimmen. Bei Biegeversuchen ist die Spannung nicht homogen über den Probenquerschnitt verteilt (MATTHECK; BETHGE 2003). Aufgrund des Flächenträgheitsmoments und der im Vergleich zur Spanplatte hohen Steifigkeit (Kapitel 5.1.2.3 und 5.1.2.2) übernimmt die außenliegende Direktbeschichtung einen Großteil der Spannungen und bestimmt maßgeblich die Biegefestigkeit (Abbildung 33). Die bei der Biegeprüfung auftretenden Schubspannungen sind aufgrund der geringen Probendicke vernachlässigbar. Es ist aktuell nicht abschätzbar, wie genau dieses Model die tatsächliche Zugfestigkeit wiedergibt. Dennoch zeigt das Model großes Potential. Es wurde keine Veröffentlichung gefunden, in der ein Weg aufgezeigt wird mechanische Kennwerte einer irreversibel aufgetragenen Beschichtung zu bestimmen. Die verringerte Festigkeit kann die erhöhte Rissanfälligkeit verursachen. Wodurch diese Festigkeitsabnahme ausgelöst wird, wird in der abschließenden Diskussion diskutiert.

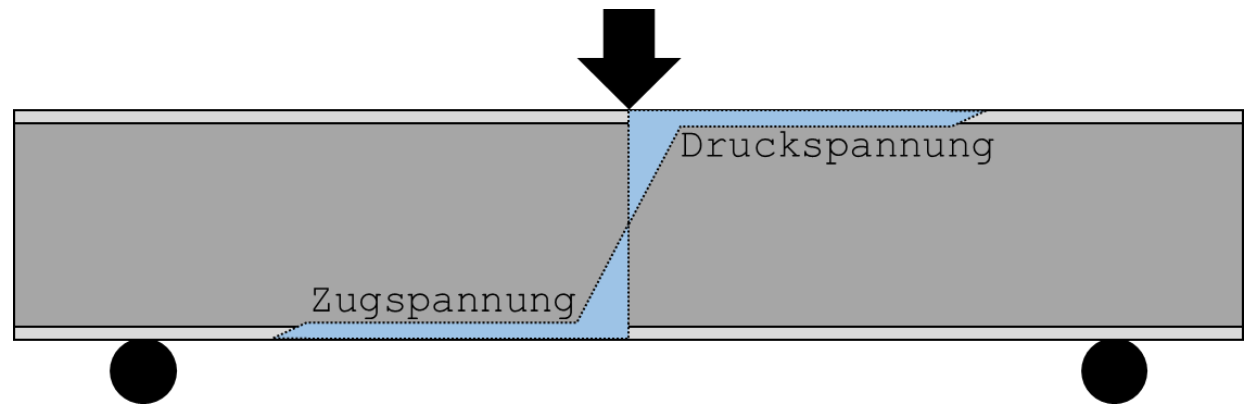

Abbildung 33: Schematischer Spannungsverlauf bei der Biegeprüfung 


\subsubsection{SPANPLATTEN}

Um zu prüfen, ob die Festigkeitsabnahme direktbeschichteter Platten durch Veränderungen der Spanplatte hervorgerufen werden, wurden Spanplatten analog eines Beschichtungsprozesses heißverpresst und Proben ( $n=6)$ im 3-Punkt-Biegeversuch geprüft.

\section{ERGEBNISSE}

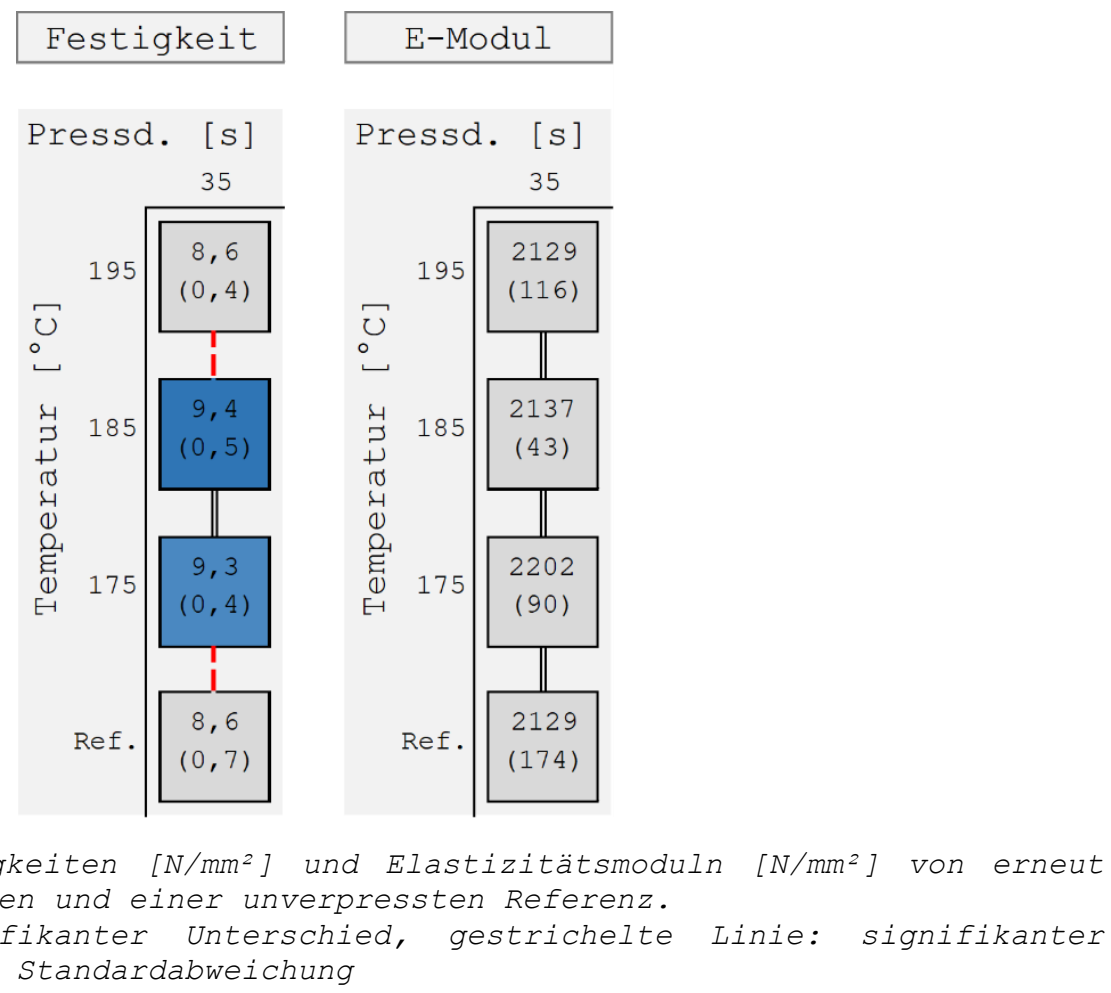

Die Spanplatten zeigen keine signifikant unterschiedlichen Elastizitäten. Bei den Biegefestigkeiten sind schwach-signifikante Unterschiede (95\% Vertrauensniveau) nachweisbar.

\section{DISKUSSION}

Die Spanplattenhersteller Rauch, Pfleiderer und Egger geben für ihre direktbeschichtbaren $16 \mathrm{~mm}$-Spanplatten Festigkeiten von $8,5 \mathrm{~N} / \mathrm{mm}^{2}$ bis $14 \mathrm{~N} / \mathrm{mm}^{2}$ und Elastizitätsmoduln von $1600 \mathrm{~N} / \mathrm{mm}^{2}$ bis $1950 \mathrm{~N} / \mathrm{mm}^{2}$ an (Rauch Holzwerkstoffe Unbekannt; Pfleiderer 2020a; Pfleiderer 2020b; EGGER 2019; EGGER 2020a; EGGER 2020b). Die Festigkeiten der hier geprüften Spanplatten liegen innerhalb dieser Spannweite. Die Elastizitätsmoduln liegen leicht darüber.

Die gemessenen Biegefestigkeiten werden als nicht signifikant unterschiedlich angesehen. Zum einen weist der paarweise Vergleich nur eine grenzwertige, schwache Signifikanz (95\%-Vertrauensniveau) auf. Grund dafür ist sowohl eine geringere Differenz zwischen den Gruppen als auch ein geringer Probenumfang. Die Signifikanz kann nicht mehr nachgewiesen werden, wenn einzelne Messwerte weggefallen. Zum anderen konnte auch OHLMEYER (2002) keine Abhängigkeit eines erhöhten Energieeintrags auf die Biegeeigenschaften nachweisen. OHLMEYER (2002) hat Spanplatten direkt nach der Produktion in Stapeln auskühlen lassen. Je nach Lage im Stapel waren sie unterschiedlichen Temperaturverläufen ausgesetzt. Biegefestigkeit und Biegemodul der Spanplatten blieben gleich. Allerdings zeigt OHLMEYER (2002) auch, dass die Querzugfestigkeit erst um bis zu $10 \%$ steigen kann und bei weiterer Belastung auf Grund von Hydrolyse auf den ursprünglichen Wert zurückfällt. Ein solcher Verlauf würde zu den gemessenen Biegefestigkeiten passen. Dennoch werden die Werte auf Grund des geringen Unterschieds als nicht signifikant angesehen. 


\subsubsection{FREIE FILME}

Um zu prüfen, ob die Festigkeitsabnahme direktbeschichteter Platten durch Veränderungen der Direktbeschichtung hervorgerufen werden, wurden freie Filme unterschiedlicher Materialien und Aushärtungsgrade $(n=10)$ im Zugversuch geprüft.

\section{ERGEBNISSE}
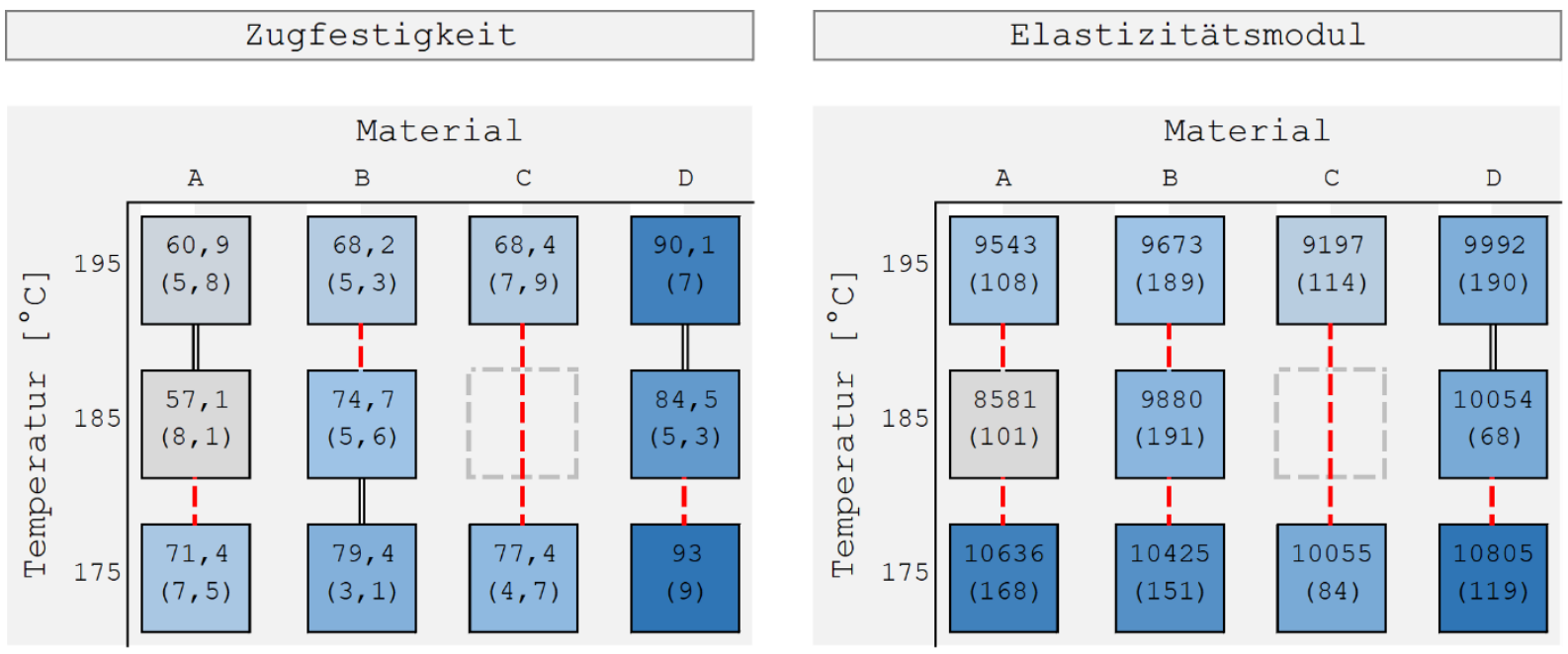

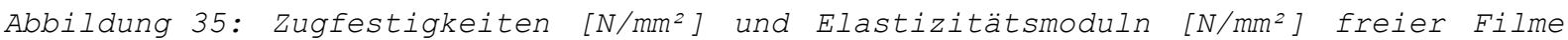
unterschiedlicher Aushärtungsgrade.

Doppellinie: kein signifikanter Unterschied, gestrichelte Linie: signifikanter Unterschied, in Klammern: Standardabweichung

A: Freier Film aus gefülltem Standardpapier \& schnell härtendem Harz,

B: Freier Film aus gefülltem Standardpapier \& langsam härtendem Harz,

C: Freier Film aus geringer gefülltem Papier \& schnell härtendem Harz,

D: Freier Film aus geringer gefülltem Papier \& langsam härtendem Harz

Es wurden freie Filme jeglicher Harz- und Papierkombinationen geprüft (Abbildung 35). Ein materialunabhängiger Zusammenhang zwischen dem mechanischen Verhalten und der Presstemperatur ist nicht vorhanden. Die bei $175^{\circ} \mathrm{C}$ produzierten Varianten weisen jedoch stets die höchsten Festigkeiten und E-Moduln auf. Alle freien Filme zeigen ein einheitliches von der Presstemperatur abhängiges Verformungsverhalten (Abbildung 36). Mit zunehmender Presstemperatur verformen sich die Filme. Bei $175^{\circ} \mathrm{C}$ gepresste Filme verdrehen sich leicht, bei $185^{\circ} \mathrm{C}$ stärker und bei $195^{\circ} \mathrm{C}$ drehen sie sich spiralig auf. 


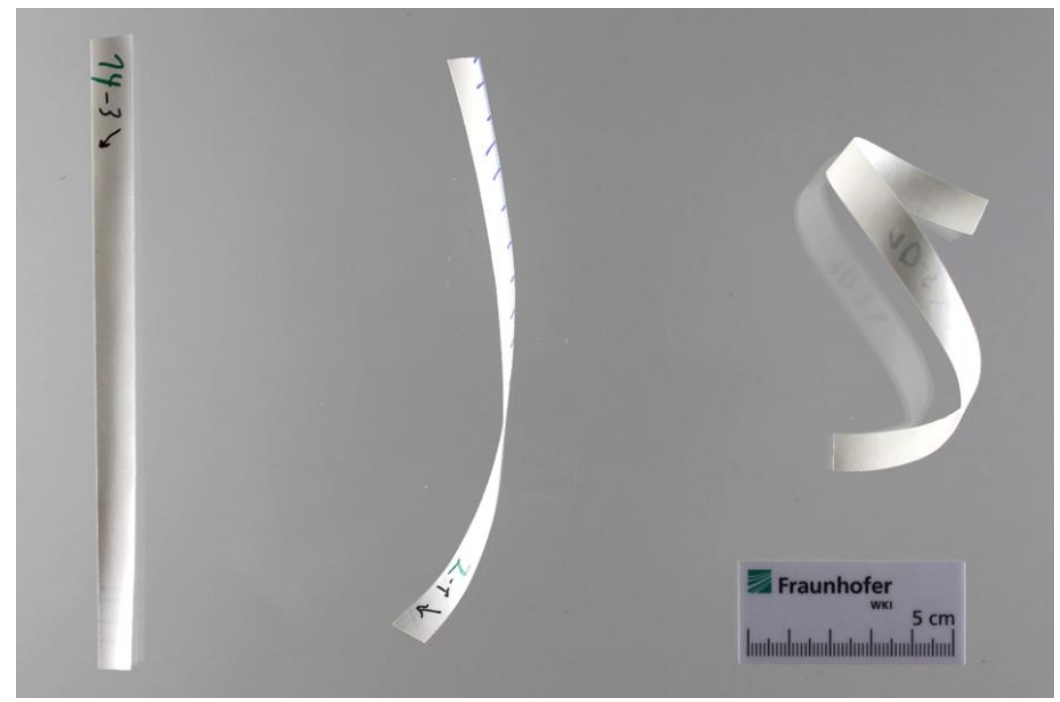

Abbildung 36: Verdrehen freier Filme unterschiedlicher Presstemperatur.

\section{DISKUSSION}

Die freien Filme wurden für die Zugprüfung aus ihrem teils stark aufgerollten Grundzustand plan gedrückt. Dies hat Auswirkungen auf die Prüfung. Einerseits werden die freien Filme durch das Plandrücken in einem Zustand geprüft, den sie als Direktbeschichtung auf einer Platte hätten. In dem Fall würden die freien Filme ebenfalls plan vorliegen. Andererseits weisen die Proben dadurch eine Vorspannung auf. Die Messwerte entsprechen daher nicht gängigen Zugfestigkeitswerten.

Literaturwerte für vergleichbare Materialien sind rar. Am ehesten kommen die Komposite aus mit Flachsfasern verstärkten Melaminharzen von HAGSTRAND; OKSMAN 2001 in Betracht. Sie haben Zugfestigkeiten von $36 \mathrm{~N} / \mathrm{mm}^{2}$ bis $60 \mathrm{~N} / \mathrm{mm}^{2}$. Allerdings lag dabei der Faseranteil mit maximal 22,2 Vol-\% geringer als bei den freien Filmen (ca. 50 Vol-\%). Deren Zugfestigkeiten liegen teilweise nah an der von Melaminharz ohne Fasern (30 N/mm² laut CZICHOS; HENNECKE (2004)). Bei den Kompositen von HAGSTRAND; OKSMAN (2001) hat demnach teilweise nur eine äußerst geringe Verstärkung durch die Flachsfasern stattgefunden. Zudem wurden Gewebe (statt Vliese wie bei den freien Filmen) verwendet. Die mechanischen Kennwerte der freien Filme geben keinen Anlass, deren Richtigkeit in Frage zu stellen.

Die Verformung der freien Filme kann vom asymmetrischen Energieeintrag beim Verpressen stammen. Bei nicht imprägnierten Papieren wird ein solches Verhalten als „Curl“ bzw. „Curling“ bezeichnet. Es tritt bei Feuchte- oder Temperaturänderungen auf wenn eine Asymmetrie entlang der Papierdicke vorliegt (HOLIK 2013), bspw. wenn ein Langsieb bei der Papierproduktion verwendet wurde. In dem Fall weist das Papier auf der Siebseite mehr Fasern und auf der gegenüberliegenden Seite mehr Füllstoffe auf. 


\subsubsection{PAPIERE}

Um zu prüfen ob die Festigkeitsabnahme direktbeschichteter Platten auch bei nicht imprägnierten Papieren nachzuweisen ist, wurden Papiere analog eines Beschichtungsprozesses heißverpresst und im Zugversuch $(n=10)$ sowie Zero-Span ( $n=15)$ geprüft. Zero-Span ist ein Zugversuch, bei dem die Klemmen direkt aneinander liegen.

\section{ERGEBNISSE}

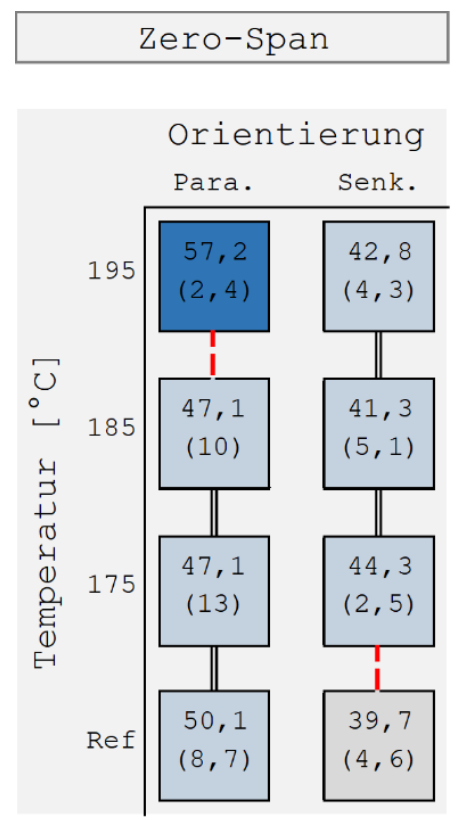

\section{Zugversuch}

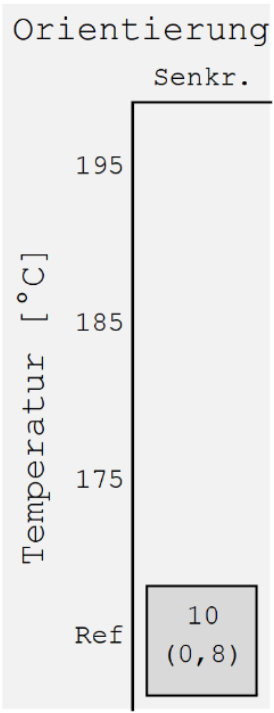

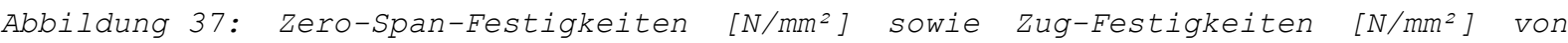
parallel sowie senkrecht zur Produktionsrichtung geprüften Papieren.

Doppellinie: kein signifikanter Unterschied, gestrichelte Linie: signifikanter Unterschied, in Klammern: Standardabweichung

Die Papiere wurden teilweise analog zur Produktion freier Film in einer Heißpresse belastet. Mittelwerte als auch Standardabweichungen der parallel zur Produktionsrichtung geprüften Proben sind überwiegend höher als bei den senkrecht entnommenen Gegenstücken (Abbildung 37). Im Zugversuch geprüft hat das Papier nur ca. 20\% der Zero-Span-Festigkeit.

Die Bruchbilder der Zugversuche unterscheiden sich (Abbildung 38). Beim Zugversuch ist die Bruchkante ausgefranst, bei der Zero-Span-Prüfung scharf abgegrenzt. 

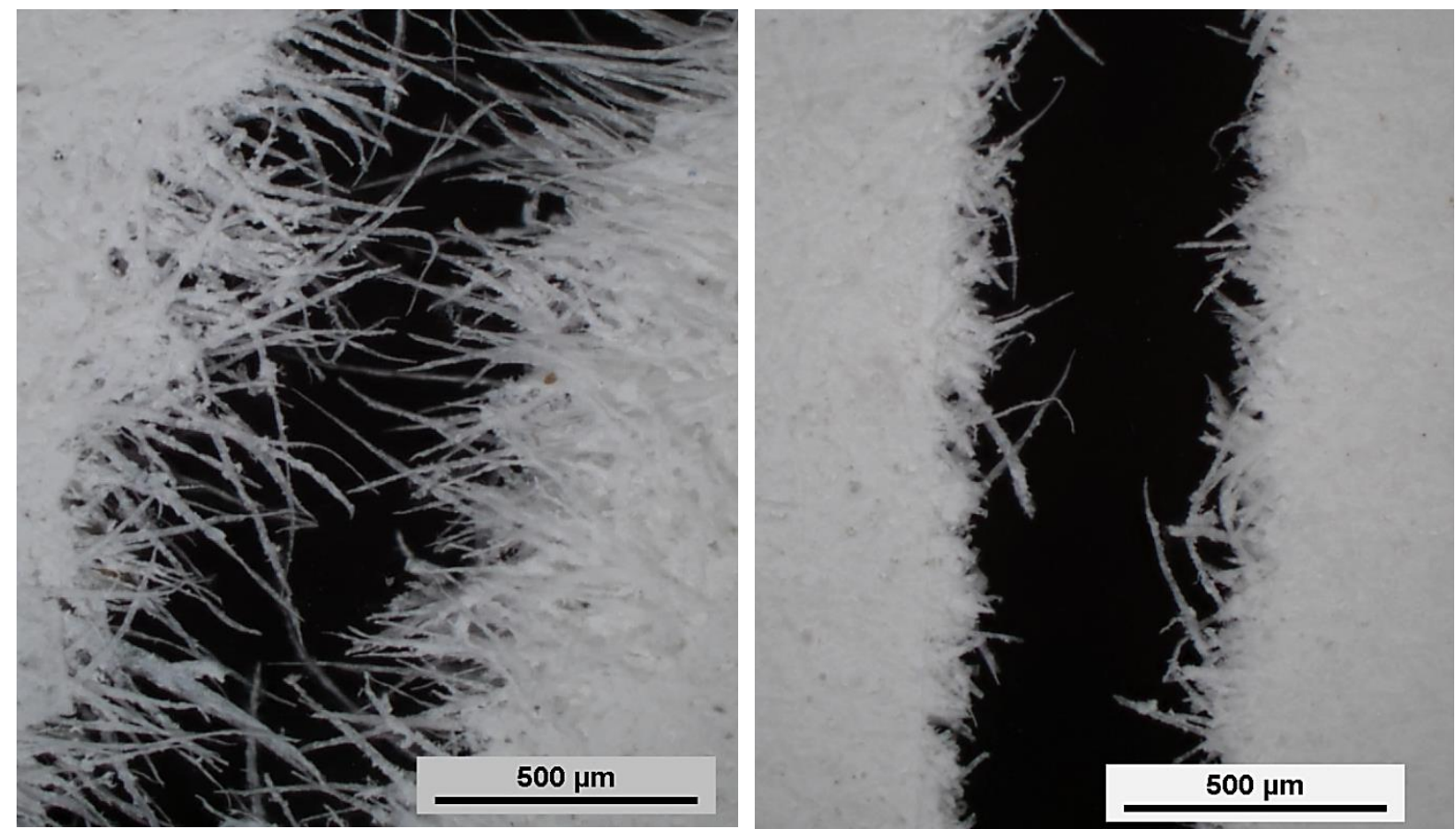

Abbildung 38: Bruchkanten von Papierproben bei einem Zugversuch (links) und einer Zero-Span-Prüfung (rechts).

\section{DISKUSSION}

Laut GuRnagUL; PAGE (1989) liegen die Reißlängen für Papier bei 2,3 km bis 14,7 km. Die Messwerte liegen mit ca. $4 \mathrm{~km}$ bis $6 \mathrm{~km}$ innerhalb dieser Spannweite.

In Produktionsrichtung des Papiers wurden höhere Zero-Span-Festigkeiten gemessen. Diese Anisotropie ist für Papiere üblich (CASEY 1980; HOLIK 2013). Grund ist eine Vorzugsorientierung der Fasern auf Grund des Produktionsprozesses. Die hohen Standardabweichungen bei parallel zur Produktionsrichtung entnommenen Proben könnten einer fehlerhaften Messung geschuldet sein. Schwankt der Druck an dem Prüfgerät, können die Messwerte variieren. Ist der Druck zu gering, rutschen die Proben aus dem Klemmen. Ist er zu hoch, werden die Fasern beschädigt. In beiden Fällen wird eine zu geringe Zero-Span-Festigkeit gemessen (BATCHELOR, et al. 2006). Auch eine heterogene Probenpräparation könnte die Messschwankungen auslösen. Eine zu spät gewechselte Skalpellklinge würde das Papier nicht mehr schneiden, sondern aufreißen. Da die Klingen nicht nach jeder Probengruppe gewechselt wurden, könnten manche Probenränder beschädigt sein. Eine mikroskopische Untersuchung zeigte jedoch keine Auffälligkeiten. Eine Beschädigung bei einer Teilgruppe von Proben erscheint dennoch logisch. Beschädigungen einzelner Proben würden die mittlere Zero-Span-Festigkeit der Gruppe herabsetzen und zu einer höheren Standardabweichung führen. Schließt man alle für eine solche Beschädigung infrage kommenden Messwerte als Ausreißer aus, gleichen sich die Festigkeiten aller parallel entnommenen Proben an. Signifikante Unterschiede sind nur noch zwischen parallel und senkrecht entnommenen Proben nachweisbar. Dies passt zu den Ergebnissen von PAGE 1969. PAGE 1969 weist zwar einen Einfluss der Temperierung auf die Zero-SpanFestigkeit von Papier nach, allerdings erst ab einer Temperierung bei ca. $200^{\circ} \mathrm{C}$ über 1 Stunde. Die Belastung in der Heißpresse ist mit $35 \mathrm{~s}$ wesentlich kürzer und die Temperatur geringer. Die Presstemperatur hat demnach keinen signifikanten Einfluss auf die Festigkeit des Papiers.

Der Unterschied zwischen der Zugfestigkeit und der Zero-Span-Festigkeit ist typisch für Vliese. Beim Zugversuch wird das Vlies auseinandergezogen. Anstatt einen Bruch zu provozieren wird der 
Widerstand beim Auseinandergleiten der Fasern gemessen. Beim Zero-Span wird ein Auseinanderziehen der Fasern unterbunden.

\subsubsection{MIKROSKOPIE}

Risse wurden mit einem Mikroskop untersucht.

\section{ERGEBNISSE}
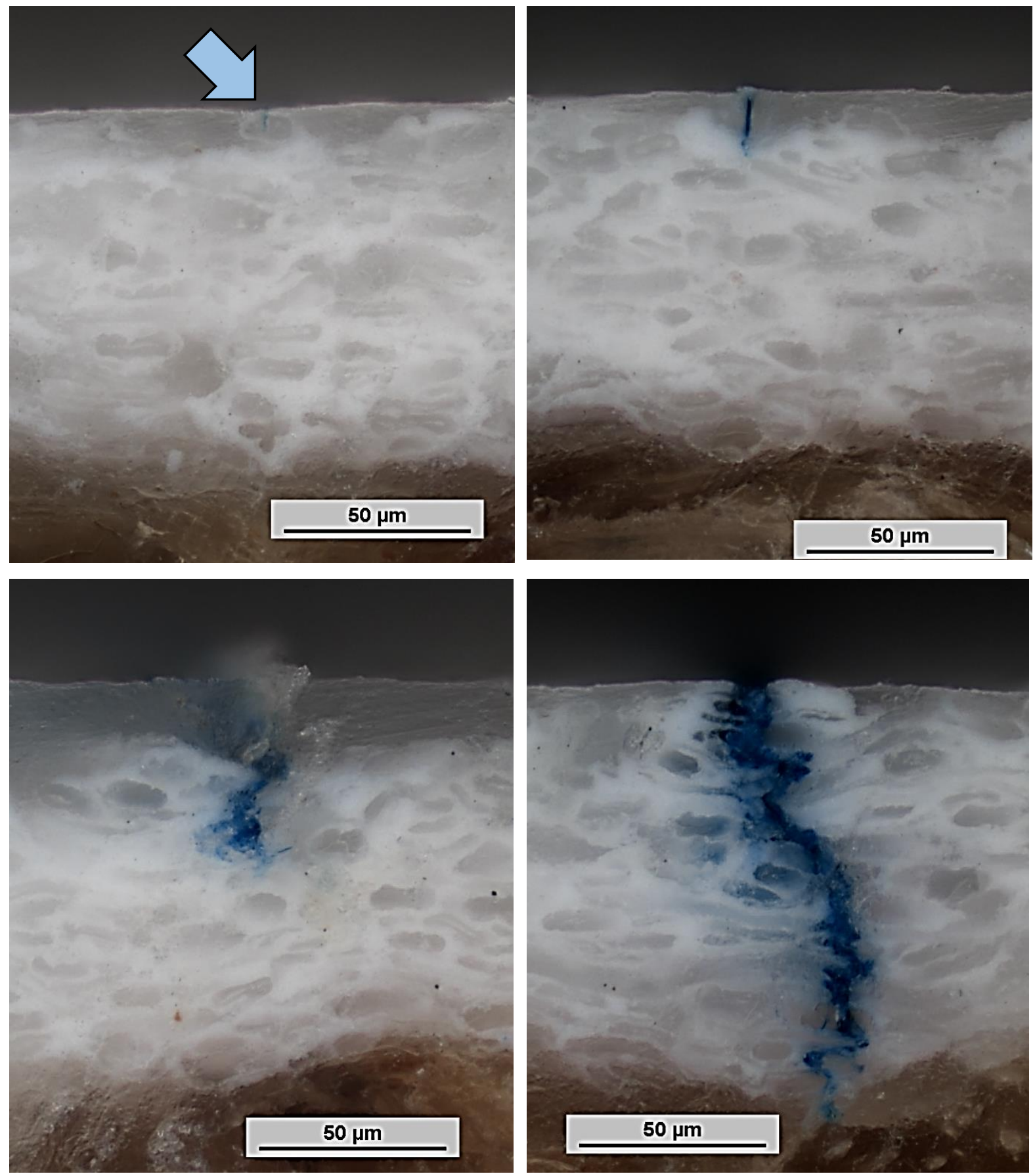

Abbildung 39: Risse unterschiedlicher Tiefen an einer stark überhärteten Probe.

Die Mikrorisse (Abbildung 39, oben links und oben rechts) sind weniger als $1 \mu \mathrm{m}$ geöffnet, Makrorisse über $6 \mu \mathrm{m}$. Der größte Riss war $1 \mathrm{~mm}$ geöffnet. Risse treten sowohl an als auch abseits von Harzseen auf. Als Harzseen werden Bereiche einer Direktbeschichtung bezeichnet, die aus Harz ohne Papierfasern bestehen. 

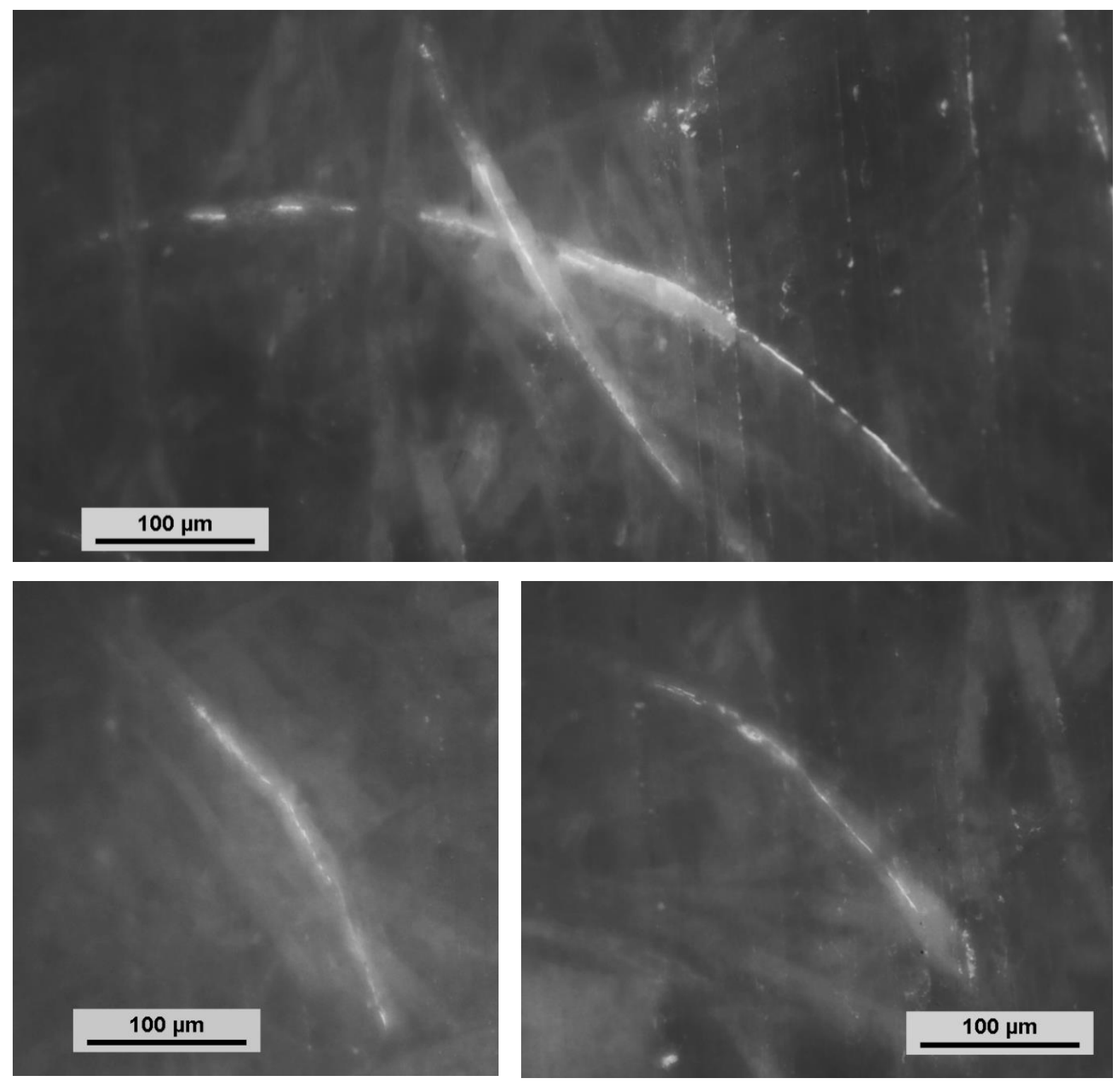

Abbildung 40: Mikroskopische Aufnahmen von Mikrorissen (UV-Fluoreszenz mit Anregung bei $365 \mathrm{~nm}$ ).

Mit Bildbearbeitung wurden die Kontraste erhöht.

Sämtliche Mikrorisse treten stets entlang oberflächennahen Papierfasern auf (Abbildung 39).

\section{DISKUSSION}

Mikrorisse treten überwiegend in der dünnen Harzauflage über Fasern auf. Je tiefer die Risse sind, desto weiter geöffnet sind sie. Größere Risse sind auch ungefärbt mit bloßem Auge sichtbar.

Das Quellen der Fasern sowie das Abkühlen der Direktbeschichtung nach der Verpressung führt zu Dimensionsänderungen. Da es sich bei Direktbeschichtungen um ein heterogenes System handelt, werden Spannungen nicht gleichmäßig verteilt. Solche Dimensionsänderungen könnten zu Spannungsspitzen und gegebenenfalls zum Einreißen der dünnen Harzschichten über oberflächennahen Fasern führen. 


\subsubsection{MIKRORISSE}

Die bei der Mikroskopie beobachteten Mikrorisse könnten die mechanischen Eigenschaften der Direktbeschichtung verändern. Es wurde daher geprüft, ob das Vorkommen der Mikrorisse mit den Festigkeitsabnahmen bei überhärteten Platten übereinstimmt. Dazu wurden angefärbte Mikrorisse unter einem Mikroskop vermessen und das Verhältnis aus kumulierter Risslänger zu untersuchter Fläche als Mikrorissdichte angegeben $(n=5)$.

\section{ERGEBNISSE}

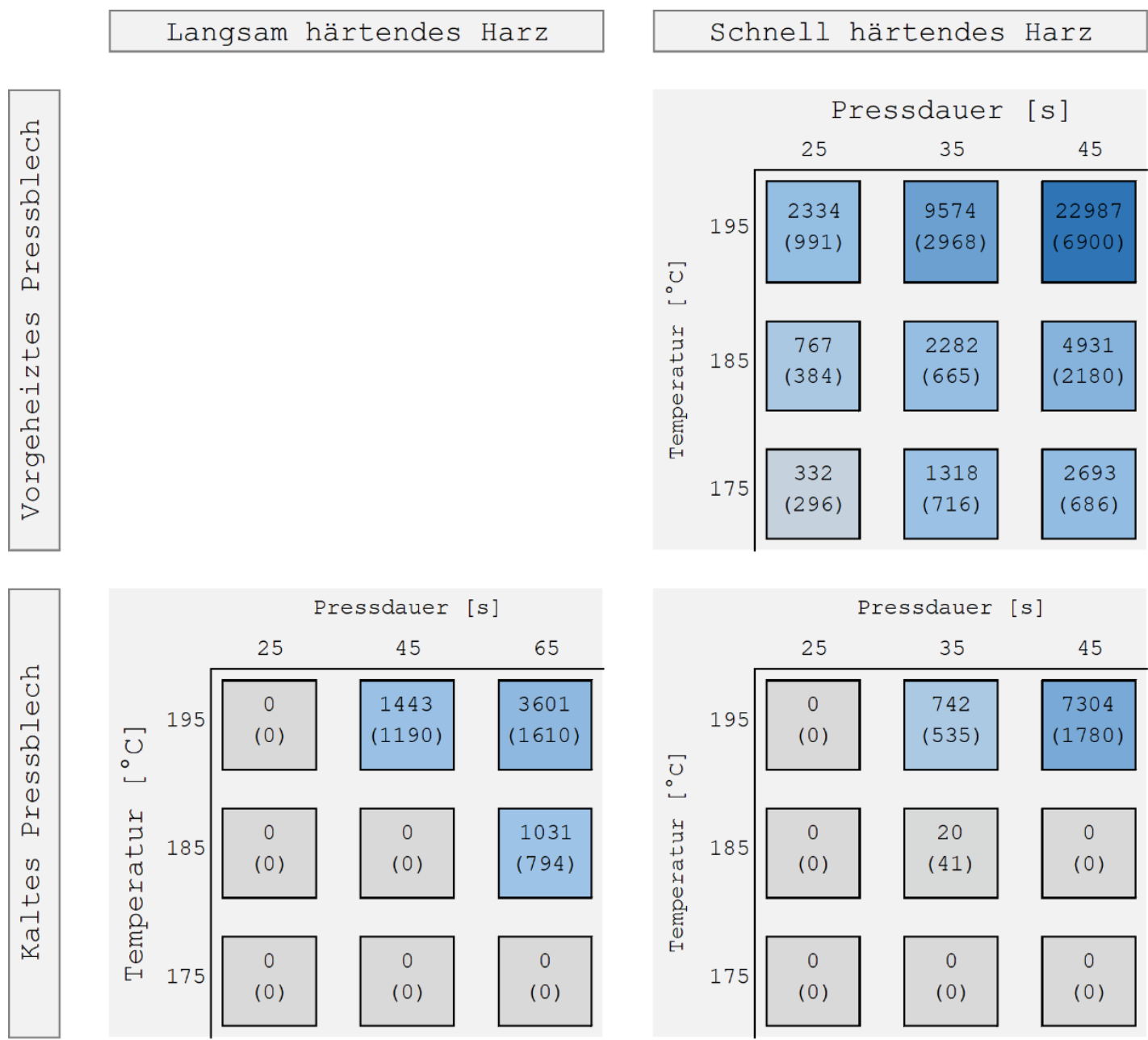

Abbildung 41: Mikrorissdichte $\left[\mathrm{\mu m} / \mathrm{cm}^{2}\right]$ direktbeschichteter Platten unterschiedlicher Aushärtungsgrade.

In den Klammern stehen die Standardabweichungen.

Erhöht man die Pressdauer oder die Presstemperatur steigt die Mikrorissdichte (Abbildung 41). Dieser Zusammenhang ist bei sämtlichen mit vorgeheiztem Pressblech beschichteten Platten ersichtlich. Bei Platten, die mit kaltem Pressblech verarbeitet wurden, sind Mikrorisse nicht oder nur im geringen Umfang entstanden. Das ANOVA-Model zu den Mikrorissen (siehe Anhang) bestätigt einen signifikanten Einfluss sowohl der Pressdauer als auch der Presstemperatur. Die Mikrorissdichten benachbarter Stellen schwanken stark (Abbildung 42).

Bei analog zu den direktbeschichteten Platten produzierten freien Film wurden keine Mikrorisse beobachtet. 


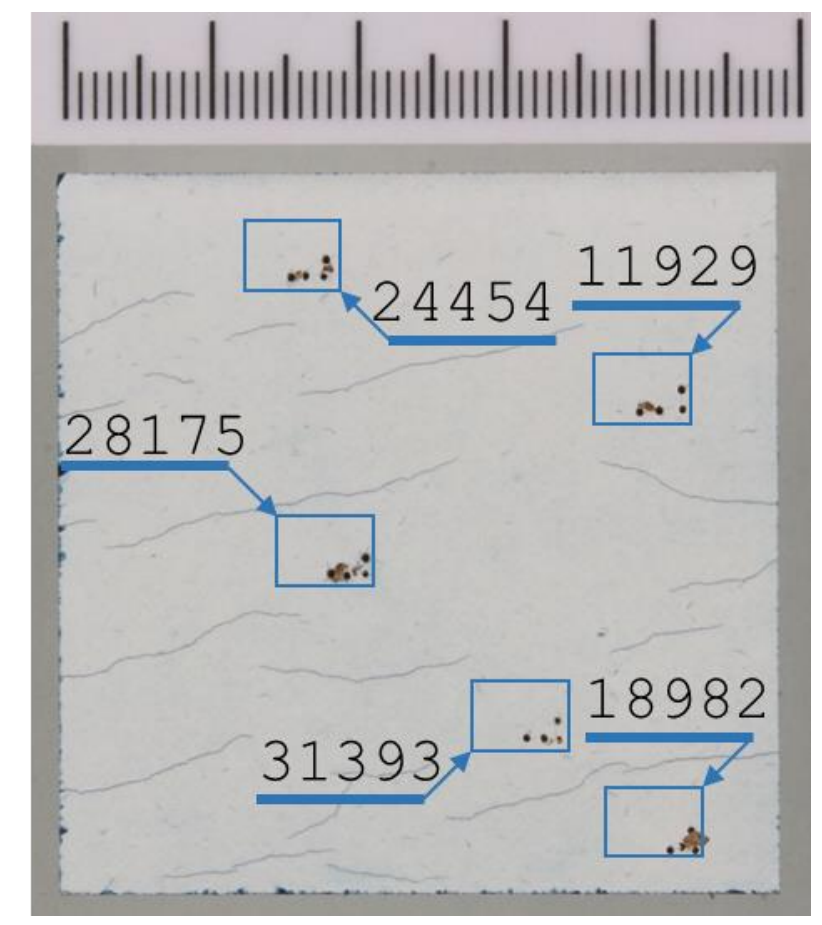

Abbildung 42: Aufnahme einer Probe mit Makrorissen und hoher Mikrorissdichte. Dargestellt sind die Areale, in denen Mikrorisse aufgenommen wurden (blaue Rechtecke) sowie die dazugehörigen Mikrorissdichten [ $\left.\mathrm{\mu m} / \mathrm{cm}^{2}\right]$

\section{DISKUSSION}

Die Standardabweichung der Mikrorissdichte ist bei sämtlichen Platten hoch. Diese Schwankungen deuten auf große lokale Unterschiede der Probenoberfläche hin (Abbildung 42). Trotz der großen Standardabweichung lässt sich ein signifikanter Zusammenhang zwischen den Pressbedingungen und der Mikrorissdichte nachweisen.

Die stark überhärtete Platte hat eine kumulierte Risslänge (Makro) von 1240 mm auf einer Oberfläche von $24,5 \mathrm{~cm} \times 24,5 \mathrm{~cm}$. Dies entspricht umgerechnet einer Makrorissdichte von $2066 \mu \mathrm{m} / \mathrm{cm}^{2}$. Die Mikrorissdichte übersteigt diesen Wert mit $22987 \mu \mathrm{m} / \mathrm{cm}^{2}$ um den Faktor 10.

Die Mikrorisse entstehen vermutlich durch Schwindungen der äußersten Schicht der Direktbeschichtung. Freie Filme rollen sich auf und bauen dadurch Spannungen ab. Als Direktbeschichtung auf einer Spanplatte wird ein Aufrollen unterbunden. Sie stehen unter Spannung und reißen leicht ein. Unbekannt ist, wann die Mikrorisse entstehen. Ob sie bereits beim Heißpressen, beim anschließenden Abkühlen oder bei der Lagerung aufkommen wurde nicht untersucht. Man könnte zu bestimmten Zeitpunkten die Oberfläche unterschiedlich einfärben. Bei der abschließenden Vermessung der Mikrorisse kann man durch die Färbung Rückschlüsse auf den Entstehungszeitpunkt ziehen.

\subsubsection{HYGRISCHES VERHALTEN}

Bei einer Überhärtung könnten die hygrischen Längenänderung der Direktbeschichtung und der Spanplatte voneinander abweichen, Spannungen zwischen den Schichten induzieren und so die erhöhte Rissanfälligkeit bewirken. Um einen solchen Einfluss zu prüfen, wurde von freien Filmen und Spanplatten das hygrische Verhalten bestimmt. 


\subsubsection{FREIE FILME}

Bei freien Filmen wurde die Längenänderung in Produktionsrichtung $(n=5)$ in Klimareihen sowie das Sorptionsverhalten $(n=1)$ in einem Klimazyklus bestimmt.

\section{ERGEBNISSE}

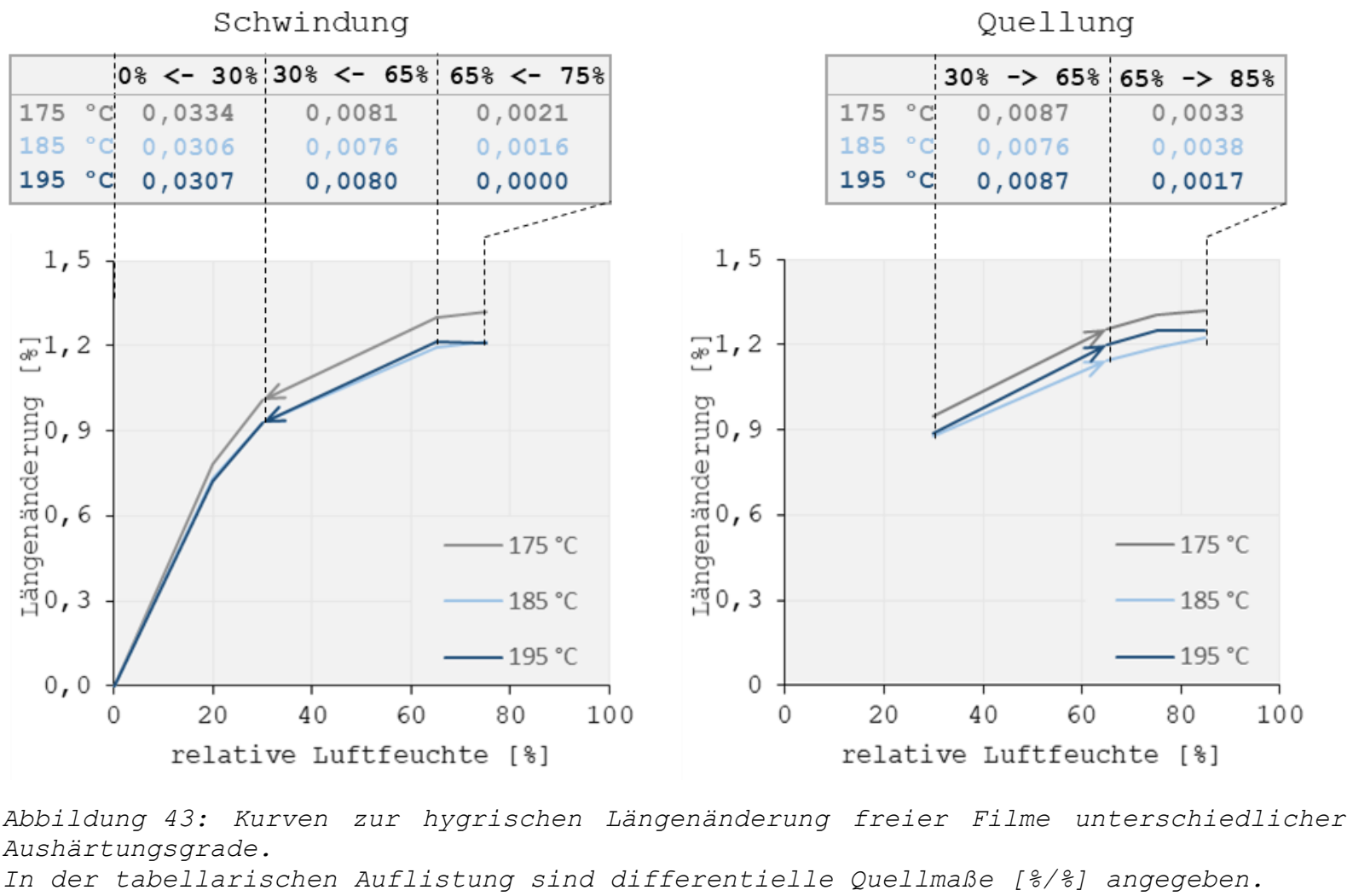

Die differentiellen Quellmaße der Proben unterscheiden sich nur gering (Abbildung 43). Im trockenen Raumklima unter $30 \%$ rel. Luftfeuchte quellen und schwinden $175^{\circ} \mathrm{C}$-Proben stärker. Über $30 \%$ rel. Luftfeuchte sind die differentiellen Quell- und Schwindmaße der freien Filme nahezu gleich.

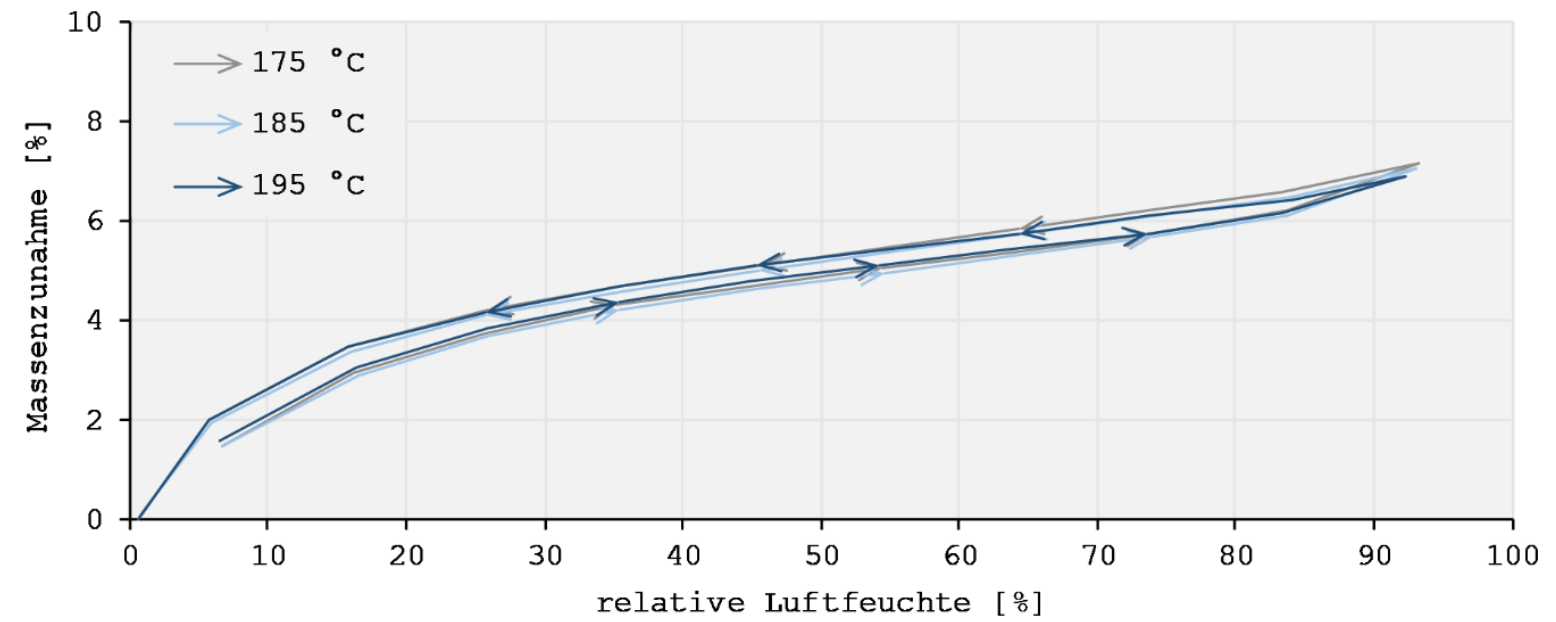

Abbildung 44: Sorption und Desorption [] von freien Filmen unterschiedlicher Aushärtungsgrade.

Das Sorptions- und Desorptionsdiagramm (Abbildung 44) zeigt das typische Verhalten eines hygrischen Materials. Unterschiede zwischen den freien Filmen sind nicht erkennbar. 


\section{DISKUSSION}

Tabelle 30: Vergleich von Literaturwerten mit den Messwerten zum hygrischen Quellen freier Filme.

\begin{tabular}{l|l|l|l} 
& $30 \%$ rel. Lf. & $65 \%$ rel. Lf. & $90 \%$ rel. Lf. \\
\hline Längenänderung & $0,7 \%$ & $0,9 \%$ & $1,1 \%$ \\
- Oefhitr (2016) & $0,9 \%$ & $1,2 \%$ & $1,2 \%$ \\
Massenzunahme & $3,5 \%$ & $5 \%$ & $7 \%$ \\
- Oehuer (2016) & $4 \%$ & $5,5 \%$ & $6,8 \%$
\end{tabular}

Scannen eignet sich durch die verzerrungsfreie Aufnahme, um die Längenänderungen aufzunehmen (LUKOWSKY 2015). OEHLER (2016) misst in einem ähnlichen Verfahren bei Melaminpapieren relative Längen- und Massenänderungen (Tabelle 30), die sich nur leicht von den hier bestimmten Messwerten unterscheiden.

Die unterhärteten freien Filme quellen und schwinden stärker. Zwei Hypothesen sprechen für einen Messfehler. Zum einen geht eine höhere hygrische Längenänderung mit einem stärkeren Sorptionsverhalten einher. Das Sorptionsverhalten der unterhärteten Proben weicht jedoch nicht von dem der anderen freien Filme ab. Zum anderen würden sich die hygrischen Längenänderungen an sämtlichen Messpunkten an die der anderen Proben angleichen, wenn deren Darrlängen um 0,1\% höher wäre. Diese vermeintlich zu geringen Darrlängen zeigen nicht nur eine Teilmenge, sondern alle $175^{\circ} \mathrm{C}$-Proben. Das kann auf einen systematischen Messfehler hindeuten. In dieser Untersuchung wurden Proben nicht randomisiert, sondern gruppiert vermessen. Messfehler wären mit den realen Längen vermischt (KLEPPMANN 2016). Ein ungenügendes Plandrücken der darrgetrockneten Proben beim Scannen könnte einen solchen Messfehler verursachen. Die freien Filme wären leicht gebogen gescannt worden und es würden fälschlicherweise zu geringe Längen gemessen.

Das hygrische Verhalten der unterhärteten Filme wird als Messfehler interpretiert. Demnach hat der Aushärtungsgrad keinen Einfluss auf das hygrische Verhalten der freien Filme. Sicher festgehalten werden kann dies jedoch derzeit nur für normal gehärtete und überhärtete Proben.

\subsubsection{SPANPLATTEN}

Spanplatten wurden zuerst analog eines Beschichtungsprozesses heißverpresst und dann deren hygrischen Längenänderungen ( $n=8)$ in Klimareihen bestimmt. 


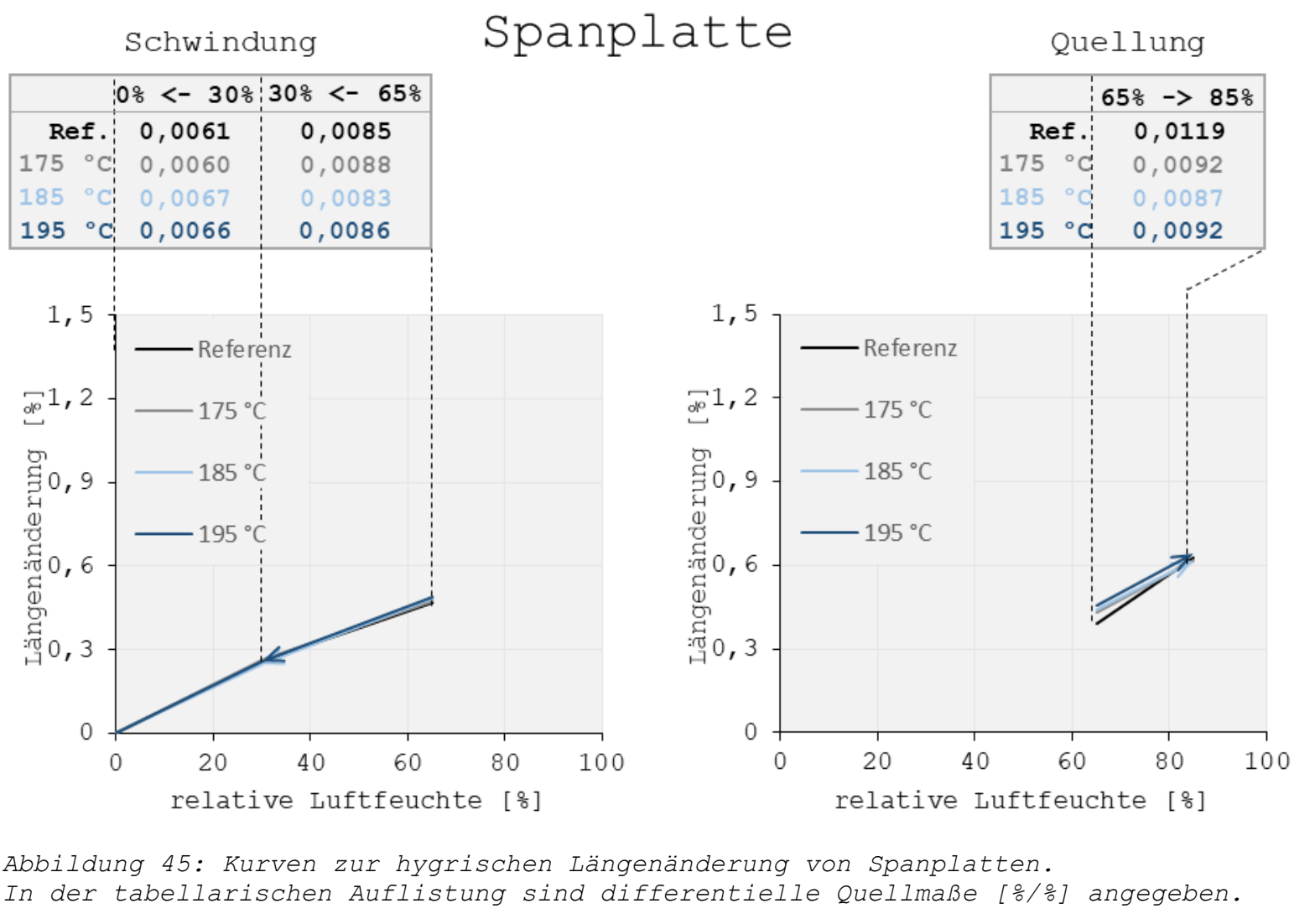

Das hygrische Verhalten der Spanplatte ist unabhängig von der erneuten Heißverpressung (Abbildung 45).

\section{DISKUSSION}

Tabelle 31: Differentielle Quell- und Schwindmaße von Spanplatten

\begin{tabular}{|c|c|c|}
\hline Quelle & $\begin{array}{l}\text { Dif. Schwindmaß }\left[\% / \frac{\circ}{0}\right] \\
(65 \%->30 \% \text { rel. Lf.) }\end{array}$ & $\begin{array}{l}\text { Dif. Quellmaß }[\% / \%] \\
(65 \%->85 \% \text { rel. Lf.) }\end{array}$ \\
\hline Messwerte & $0,008-0,009$ & $0,009-0,012$ \\
\hline ROFFAEL $\quad(2004)$ & 0,005 & 0,01 \\
\hline Thole $\quad$ (1991) Thole 1991 & $0,003-0,004$ & $0,007-0,01$ \\
\hline SONDEREGGER; NIEMZ (2006) & 0,036 & 0,049 \\
\hline SchWAa; StefFen; Korte (1997) & $0,002-0,003$ & $0,006-0,007$ \\
\hline
\end{tabular}

Die Schwindmaße für den Bereich 65\% bis 30\% rel. Lf. liegen über Literaturwerten (Tabelle 31). Die hohen Werte sind möglicherweise auf den Verwendungszweck der Spanplatten zurückzuführen. Bei den hier untersuchten Platten handelt es sich nicht um Spanplatten für den „allgemeinen Zweck“, wie sie in der Literatur untersuchten wurden. Stattdessen wurden für Direktbeschichtung produzierte Spanplatten verwendet. Inwiefern sich diese von Spanplatten für den allgemeinen Zweck unterscheiden ist nicht klar. Durch die höheren Schwindmaße liegen diese gleichauf mit denen freier 
Filme. Ob das Schwindmaß der Spanplatten absichtlich erhöht wurde, um mit denen der Direktbeschichtungen übereinzustimmen, ist jedoch fraglich. Zwar sind gleiche Expansionskoeffizienten von aufeinanderliegenden Schichten auf Grund der dadurch vorliegenden Spannungsarmut erstrebenswert. Allerdings muss dafür das hygrische Verhalten der Direktbeschichtungen bekannt sein. Veröffentlichungen mit entsprechenden Angaben wurden nicht gefunden. Firmeninterne Untersuchungen können nicht ausgeschlossen werden. 


\subsubsection{DIFFUSIONSWIDERSTAND}

Eine diffusionsoffene Direktbeschichtung könnte zu ausgeprägtem Quellen und Schwinden der Spanplatte führen. Das könnte Spannungen zwischen der Beschichtung und der Spanplatte induzieren und die hohe Rissanfälligkeit bei Überhärtung hervorrufen. Um einen entsprechenden Einfluss der Aushärtungsbedingungen auf den Diffusionswiderstand zu prüfen, wurden freie Filmen $(n=5)$ mittels DryCup und WetCup geprüft. Da dabei viele Proben kaputtgingen, wurden anschließend direktbeschichtete Platten geprüft. Dazu wurden Proben $(n=3)$ klimatisiert, Ränder mit Silikon abgedichtet und der Masseverlust nach einer Trocknung bestimmt.

\section{ERGEBNISSE}
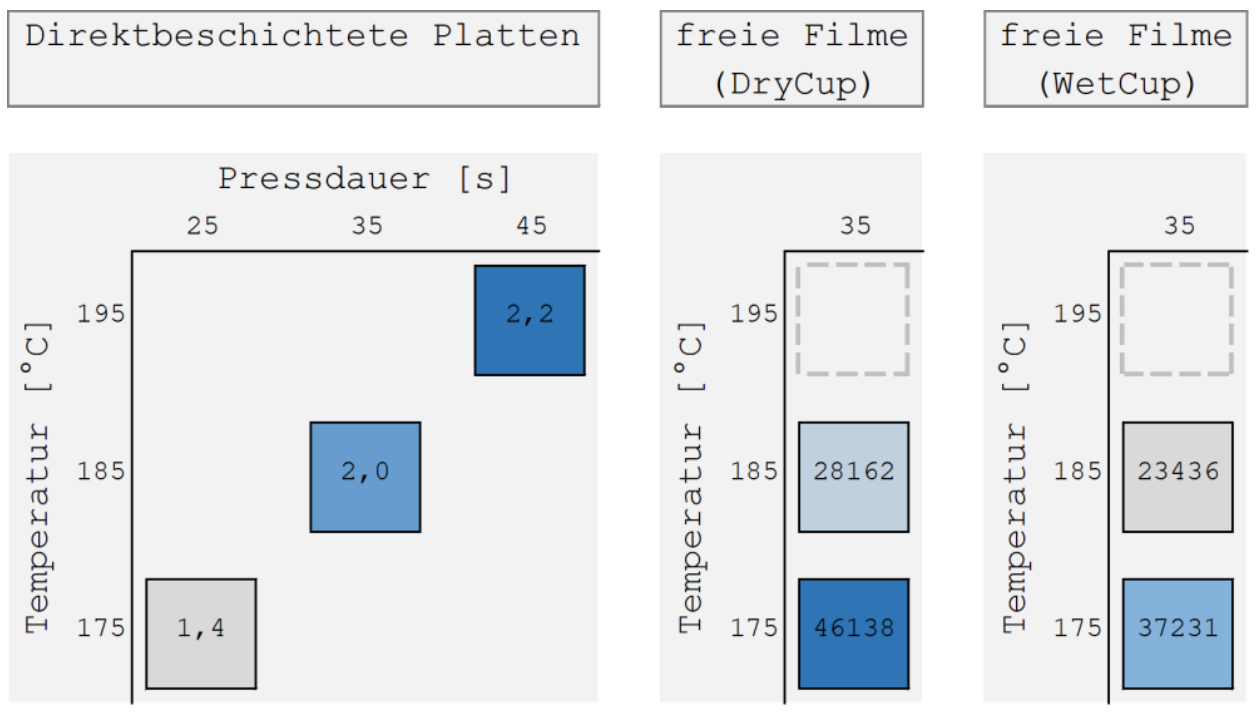

Abbildung 46: Diffusionsverhalten direktbeschichteter Platten und freier Filme. Die Angabe zu den Platten entspricht dem prozentualen Massenverlust bei Trocknung. Bei den freien Filmen sind die $\mu$-Werte angegeben.

Der Diffusionswiderstand ist abhängig vom Aushärtungsgrad. Überhärtete Platten verlieren in derselben Trocknungsphase mehr Masse als geringer gehärtete. Bei DryCup und WetCup sind viele freie Filme gerissen und konnten nicht vermessen werden. Die Werte für die $185^{\circ} \mathrm{C}$ und $175^{\circ} \mathrm{C}$ Proben zeigen ein Verhalten analog zur Prüfung an den direktbeschichteten Platten; unterhärtete Proben mit einem hohen Diffusionswiderstand, optimal gehärtete mit einem niedrigeren.

\section{DISKUSSION}

SONDEREGGER; NIEMZ (2009) berechnen Diffusionskoeffizienten für Spanplatten und direktbeschichtete Platten. Aus deren Messwerten lässt sich ein sd-Wert (DryCup) der Direktbeschichtung von ca. $5 \mathrm{~m}$ ableiten. Der sd-Wert der freien Filme liegt mit ca. 3,0 m bis 4,6 m in einem ähnlichen Rahmen.

Bei einem stark vernetzten Material ist ein höherer Diffusionswiderstand zu vermuten. Die Diffussionswiderstände der freien Filme und der direktbeschichteten Platten nehmen jedoch mit steigender Vernetzung ab. Mikrorisse lassen sich als Ursache ausschließen. Zwar wurden bei den direktbeschichteten Platten Mikrorisse gefunden, deren Umfang in Abhängigkeit zum Aushärtungsgrad steht: überhärtete Proben haben viele, unterhärtete wenige Mikrorisse. Allerdings zeigen freie Filme ein analoges Diffusionsverhalten trotz Abwesenheit von Mikrorissen. Wenn ein Einfluss der Mikrorisse auf das Diffusionsverhalten besteht, ist dieser nicht maßgeblich für das hier gemessene Verhalten. Die Ursache für den abnehmenden Diffusionswiderstand wurde nicht gefunden. 


\subsubsection{EIGENSPANNUNGEN}

Spannungen im System können zum Materialversagen führen. Es sollte geprüft werden, ob bei Proben mit einer hohen Rissanfälligkeit eine hohe Eigenspannung vorliegt. Dazu wurden Proben $(n=5)$ bestehend aus Spanplattendeckschicht und Direktbeschichtung erst bei 30\% rel. Lf., dann 85\% rel. Lf. klimatisiert und das Stichmaß der verformten Proben als Maß für deren Eigenspannung vermessen.

\section{ERGEBNISSE}

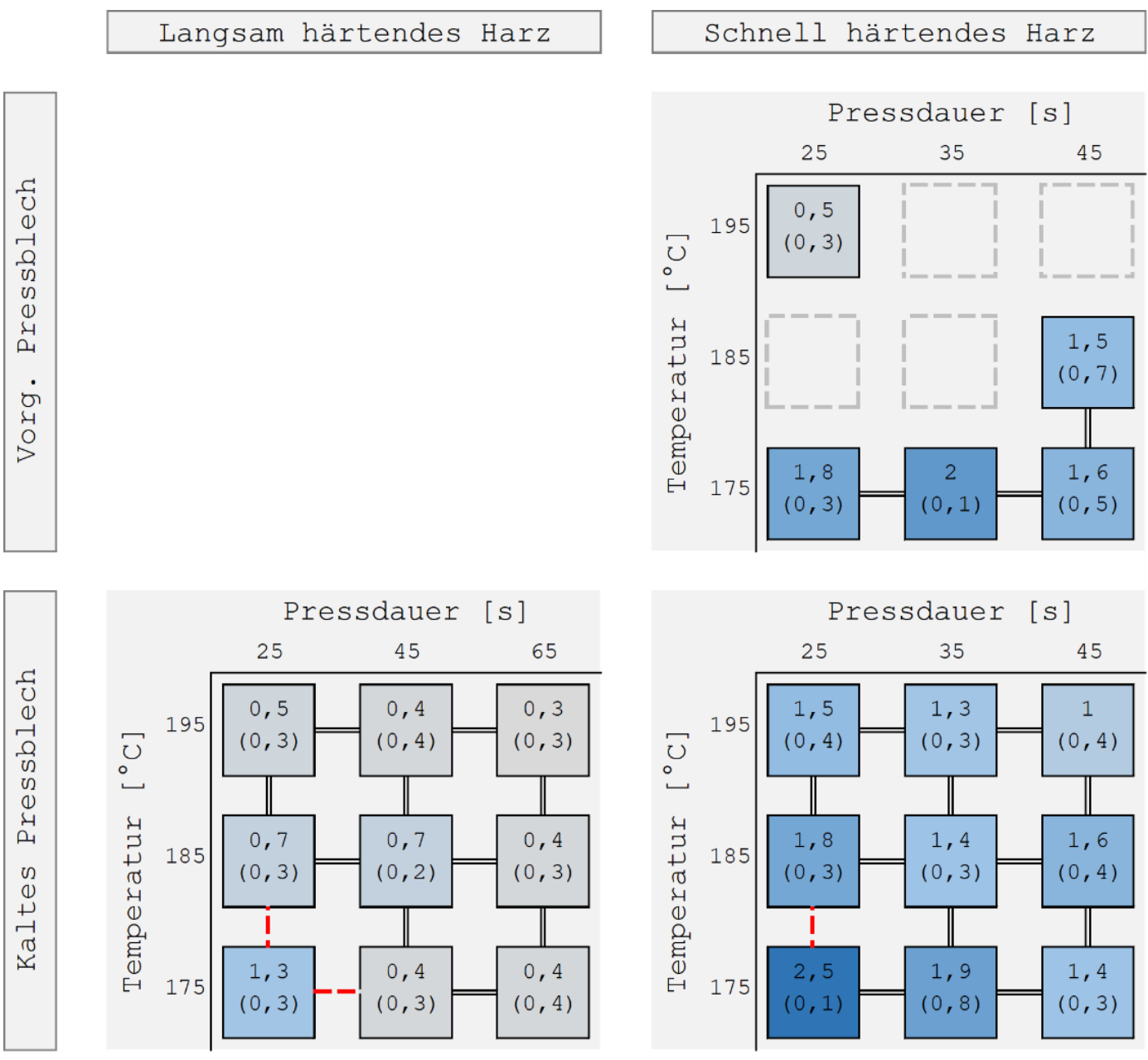

Abbildung 47: Änderungen des Stichmaßes [mm], wenn die relative Luftfeuchte von 30 auf 85 erhöht wird.

Doppellinie: kein signifikanter Unterschied, gestrichelte Linie: signifikanter Unterschied, in Klammern: Standardabweichung

Beim Wechsel von $30 \%$ rel. Lf. zu $85 \%$ rel. Lf. verändern sich überhärtete Proben nur gering (Abbildung 47). Sämtliche Proben zeigen bei 85\% rel. Lf. höhere Stichmaße. Die Proben des langsam härtenden Harzes sind schwächer verformt.

Die Probendicke schwankt um ca. 0,1 mm. An manchen Proben kommen große Späne der Spanplattenmittelschicht an der Schnittkante vor.

\section{DISKUSSION}

Die verwendete Methode wird von LUKOWSKY (2015) zur Abschätzung von Eigenspannungen bei Melaminharzbeschichtung vorgeschlagen. Die Methode weist jedoch undefinierte Einflussgrößen auf. Der Probenzuschnitt erfolgt tendenziell am Übergang der Deckschicht zur Mittelschicht. Da die Probendicke schwankt, können Späne der Spanplattenmittelschicht an der Probe anhaften und die Verformung verändern. 
Die Stichmaße bei 30\% und 85\% rel. Lf. zeigen Spannungen in den Proben, die unabhängig vom Aushärtungsgrad sind. Es lässt sich lediglich eine Abhängigkeit zum Klima und zum Harz ablesen. Im trockenen Klima sind die Stichmaße geringer als im darauffolgenden feuchten Klima. Die Spanplatte dehnt sich beim Klimawechsel mehr aus als die Direktbeschichtung. Dies passt zu den gemessenen hygrischen Längenänderungen freier Filme und Spanplatten für relative Luftfeuchten über $65 \%$.

Die Stichmaßänderung beim Klimawechsel ist hingegen abhängig von Pressdauer und -Temperatur; niedrige Stichmaßänderungen bei hohen Aushärtungsgraden. Eine Abhängigkeit der hygrischen Längenänderung der freien Filme oder Spanplatten zum Aushärtungsgrad würde dies erklären. Es wurde jedoch bewiesen, dass eine solche Abhängigkeit bei freien Filmen und Spanplatten nicht existiert. Allerdings könnte das hygrische Verhalten freier Filme auf Grund fehlender Mikrorisse nicht repräsentativ für das der Direktbeschichtung sein. Neben einen Einfluss auf die relative Längenänderung, könnten die Mikrorisse auch die Verformung beeinflussen. Anhand der Verformungsrichtung der Stichmaßproben ist zu erkennen, dass die Direktbeschichtung unter Zugspannung steht. Bei überhärteten Proben könnten sich die zahlreichen Mikrorisse unter der Zugspannung öffnen. Die Verformung würde geringer ausfallen. Unterhärtete Proben können aufgrund der geringen Mikrorissdichte Spannungen nicht auf diese Weise abbauen. Sie verformen sich beim Klimawechsel stärker.

Sollten Eigenspannungen in einer späteren Arbeit untersucht werden, empfiehlt es sich statt einer $16 \mathrm{~mm}$ dicken, dreischichtigen Spanplatte, eine dünne, einschichtige Spanplatte zu beschichten. Zum einen entfällt der Dickenzuschnitt bei der Probenpräparation. Eine potenzielle Störgröße fällt weg. Zum anderen ist die empfohlene Spanplatte deutlich homogener hinsichtlich Spanverteilung und Dichte. 


\subsubsection{AUSHÄRTUNGSGRAD}

Die Rissanfälligkeiten des langsamen und des schnell härtenden Harzes unterscheiden sich teils deutlich. Um zu prüfen, ob dies nur auf nicht vergleichbare Aushärtungen zurückzuführen ist, wurden die Aushärtungsgrade bestimmt. Dazu wurde von der Beschichtung geschliffenes Pulver ( $n=10)$ mit Salzsäure hydrolysiert und die Menge hydrolysierten Melamins mittels UV-Spektrometer bestimmt.

\section{ERGEBNISSE}

Langsam härtendes Harz
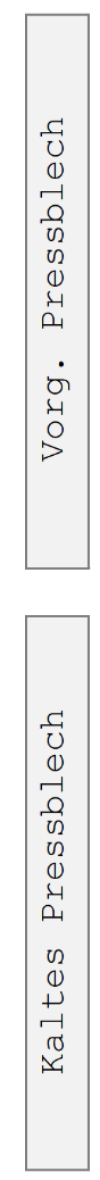

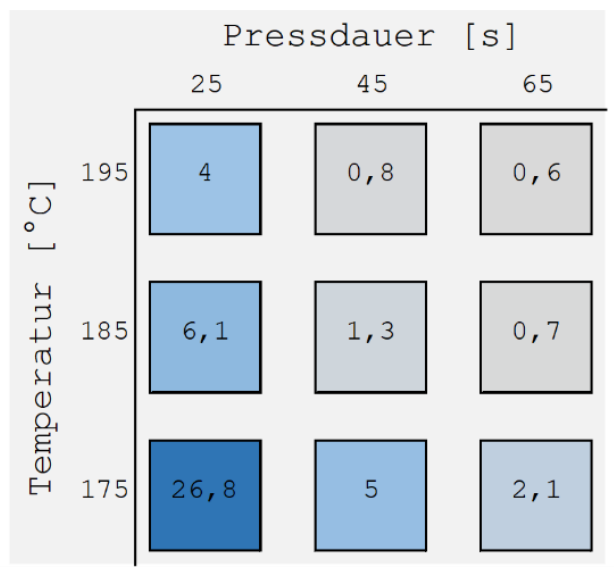

Schnell härtendes Harz
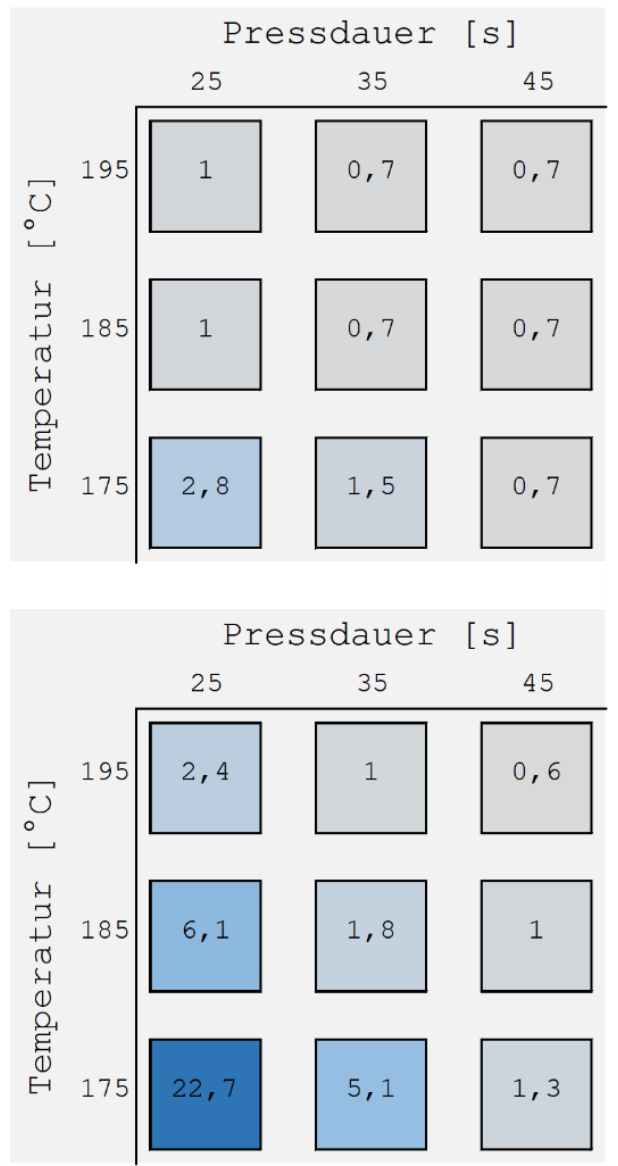

Abbildung 48: Durch Pulverhydrolyse gelöstes Melamin [mg/l] direktbeschichteter Platten unterschiedlicher Aushärtungsgrade.

Presstemperatur und -dauer bestimmen die Konzentration des Melamins in der Hydrolyselösung. Je höher der Energieeintrag beim Verpressen (je länger die Presse geschlossen bzw. je höher die Presstemperatur eingestellt ist) desto weniger Melamin kann bei der Hydrolyse aus der Beschichtung gelöst werden.

Der Aushärtungsgrad ist zudem abhängig vom Harz. Beim schnell härtenden Harz konnte bei denselben Pressbedingungen weniger Melamin ausgelöst werden als beim langsam reagierenden Harz. Wird das schnell härtende Harz mit einem vorgeheizten Pressblech produziert, sind die Messwerte der unterhärteten Proben geringer.

\section{DISKUSSION}

Überhärtete Proben sind mit der Methode kaum noch zu differenzieren. Unterhärtete Proben hingegen lassen sich auf Grund der großen Spannweite der Messwerte gut unterscheiden. Mit zunehmender Aushärtung sinkt die Anzahl weiterer Bindungsmöglichkeiten. Das Harz verändert sich 
chemisch immer weniger (KANDELBAUER, et al. 2009a). Analog sinkt die Differenzierung des Aushärtungsgrads. Auf Grund der Abhängigkeit des Aushärtungsgrades vom Harz lassen sich keine allgemeingültigen Grenzen ziehen, die eine Über- bzw. Unterhärtung aufzeigen. Die Methode eignet sich nur für vergleichende Aussagen.

Die unterschiedlichen Rissanfälligkeiten der beiden Harztypen lassen sich nicht mit unterschiedlichen Aushärtungsgraden begründen. Die Spannweiten der Aushärtungsgrade überschneiden sich. 


\subsubsection{ABSCHLIEßENDE DISKUSSION ZUR VERSUCHSREIHE}

Die kumulierten Risslängen sind abhängig von den Aushärtungsbedingungen. Je höher Pressdauer und -Temperatur, desto rissanfälliger ist die Platte. Eine Rissanfälligkeit kann durch eine Festigkeitsabnahme, veränderte hygrische Längenänderungen und Diffusionswiderstände sowie höhere Eigenspannungen begründet sein. Im Folgenden werden Hypothesen dazu diskutiert.

\section{FESTIGKEITSABNAHME}
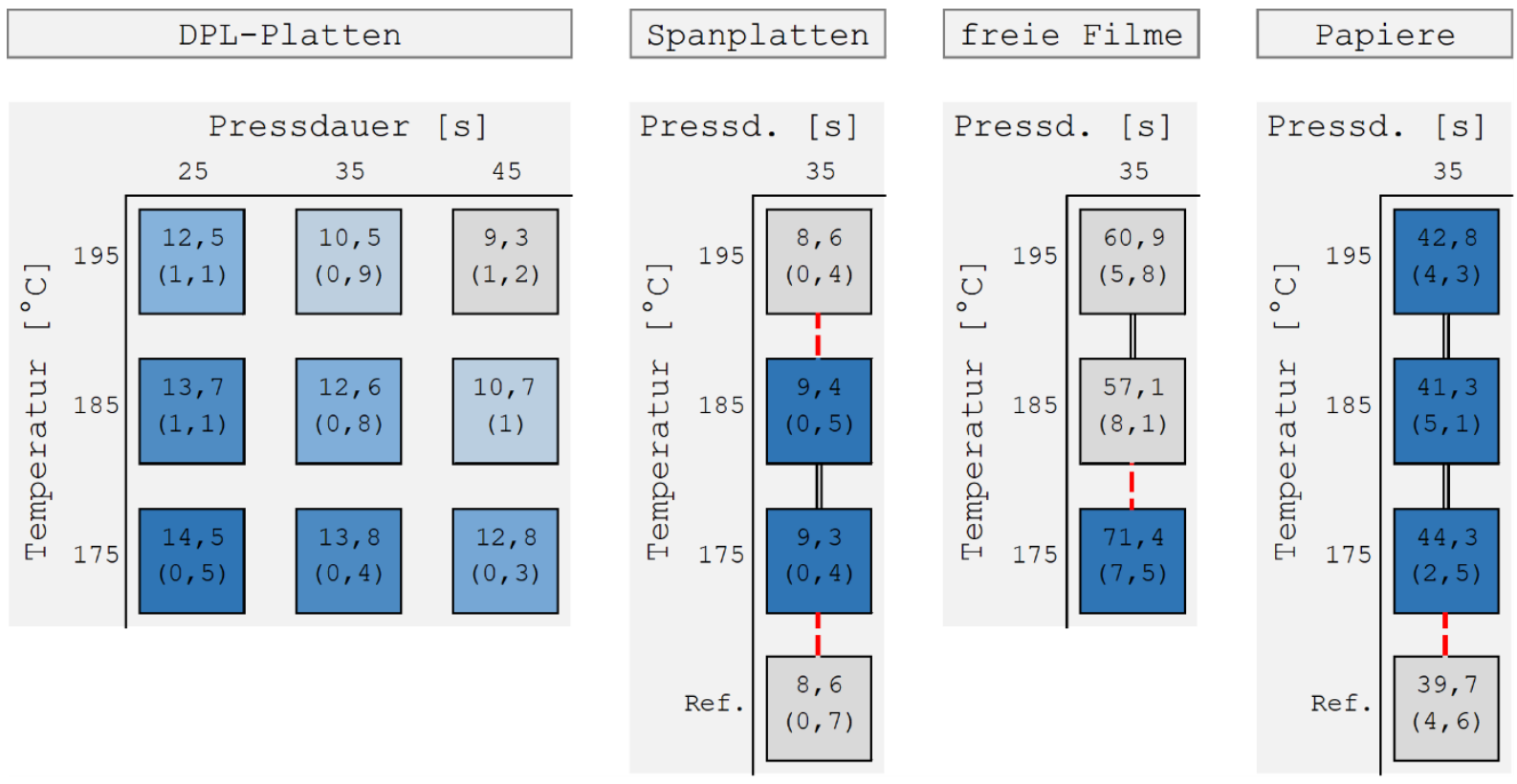

Abbildung 49: Wiederholung der Biegefestigkeiten [N/ $\left.\mathrm{mm}^{2}\right]$ direktbeschichteter Platten und Spanplatten, der Zugfestigkeiten [N/ $\left.\mathrm{mm}^{2}\right]$ freier Filme als auch der Zero-SpanFestigkeit $\left[\mathrm{N} / \mathrm{mm}^{2}\right]$ der Papiere.

Doppellinie: kein signifikanter Unterschied, gestrichelte Linie: signifikanter Unterschied, in Klammern: Standardabweichung

Platten mit hohen kumulierten Risslängen haben geringe Biegefestigkeiten. Im Falle der stark überhärteten Platte sinkt die Biegefestigkeit fast auf die einer unbeschichteten Spanplatte. Der Zusammenhang zwischen Biegefestigkeit und Rissanfälligkeit ist nicht zufällig. Bei einem Biegeversuch ist die äußerste Schicht ausschlaggebend für die Festigkeit (MATTHECK; BETHGE 2003). Die Biegefestigkeit von direktbeschichteten Platten hängt daher maßgeblich von der Festigkeit der Direktbeschichtung ab. Bei der Rissanfälligkeitsprüfung wird eine Spannung zwischen der Direktbeschichtung und der Spanplatte induziert. Bei optimal gehärteten Platten ist die Festigkeit der Direktbeschichtung hoch und die Spannungen führen nicht zum Materialversagen. Bei überhärteten Platten überschreiten die Spannungen die Festigkeiten der Direktbeschichtung und Risse entstehen.

Die Verringerung der Biegefestigkeit sollte auf Veränderungen einzelner Komponenten der direktbeschichteten Platte zurückgeführt werden können (Abbildung 49). Es wurde nachgewiesen, dass die Festigkeit der Spanplatte und des Papiers unverändert bleibt. Ein Einfluss dieser Materialien auf die Biegefestigkeit und dadurch auf die Rissanfälligkeit ist daher auszuschließen. Die Zugfestigkeiten und Elastizitätsmoduln der Direktbeschichtungen lassen sich aus den Biegefestigkeiten mittels eines mathematischen Models ableiten. Offenbar wurde der verwendete Ansatz dazu hier erstmals verwendet. Die berechneten Zugfestigkeiten zeigen eine zur Rissanfälligkeit passende signifikante Abnahme. Ein analoges Verhalten konnte allerdings bei den Zugprüfungen freier Filmen nicht nachgewiesen werden. Zwar zeigen die freien Filme einen signifikanten Festigkeitsabfall bei Erhöhung der Presstemperatur von $175^{\circ} \mathrm{C}$ auf $185^{\circ} \mathrm{C}$. Bei $195{ }^{\circ} \mathrm{C}$, der Presstemperatur bei der die 
Rissanfälligkeit erhöht ist, zeigt sich jedoch keine weitere Veränderung. Der Festigkeitsabfall der Direktbeschichtung kann nicht mit der Zugfestigkeit der freien Filme erklärt werden.
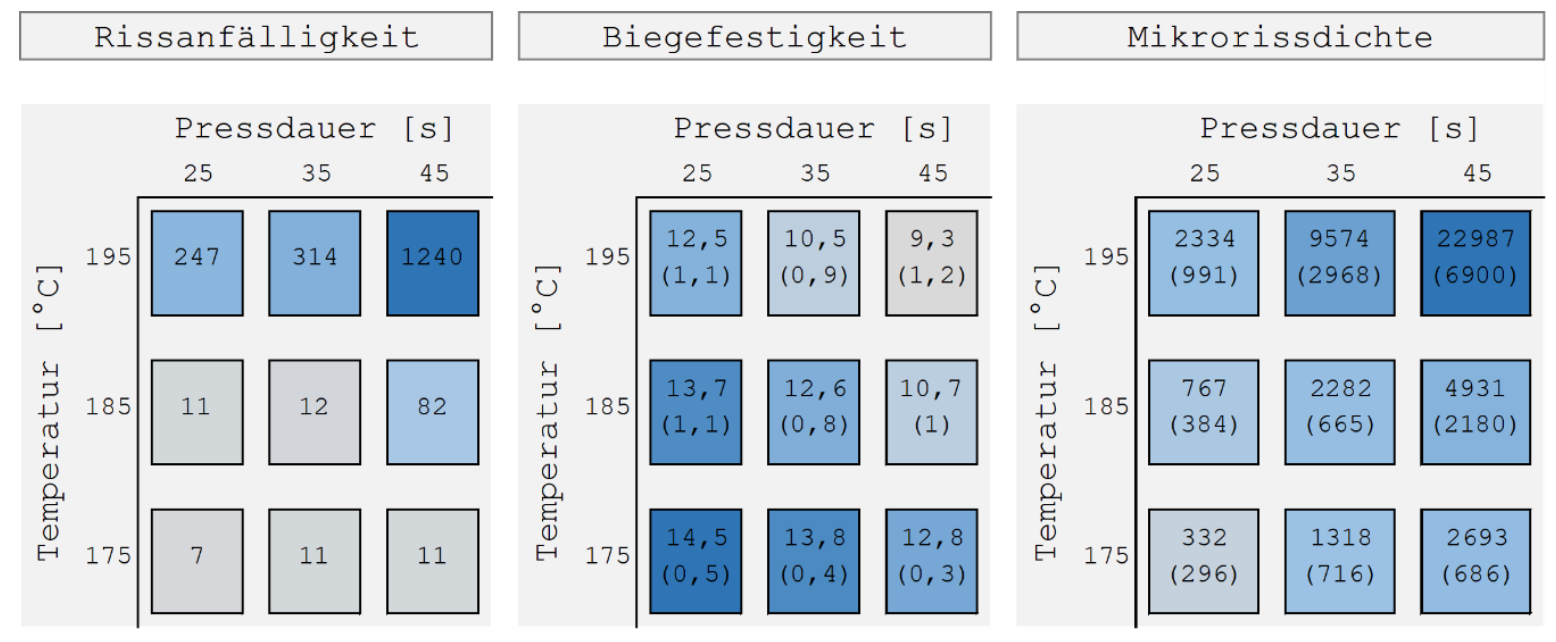

Abbildung 50: Wiederholung der Rissanfälligkeit [mm], Biegefestigkeiten [N/mm²] und Mikrorissdichte [ $\left.\mathrm{mm} / \mathrm{cm}^{2}\right]$ derselben direktbeschichteten Platten.

In den Klammern stehen die Standardabweichungen.

Allerdings unterscheiden sich freie Filme in einem wesentlichen Punkt von Direktbeschichtungen: bei Direktbeschichtungen kommen Mikrorisse vor (Abbildung 50), bei freien Filmen nicht. Mikrorisse führen zu einer Materialschwächung, die Festigkeit nimmt ab (GRIFFITH 1921; MANG; HOFSTETTER 2018). Dieser Zusammenhang zwischen Mikrorissdichte und Festigkeit ist in Abbildung 51 dargestellt. Bei der stark überhärteten Platte sinkt die Biegefestigkeit sogar auf den Wert einer unbeschichteten Spanplatte. Ab einer Mikrorissdichte von ca. $5000 \mu \mathrm{m} / \mathrm{cm}^{2}$ führen weitere Mikrorisse nur noch zu einer geringen Abnahme der Festigkeit.

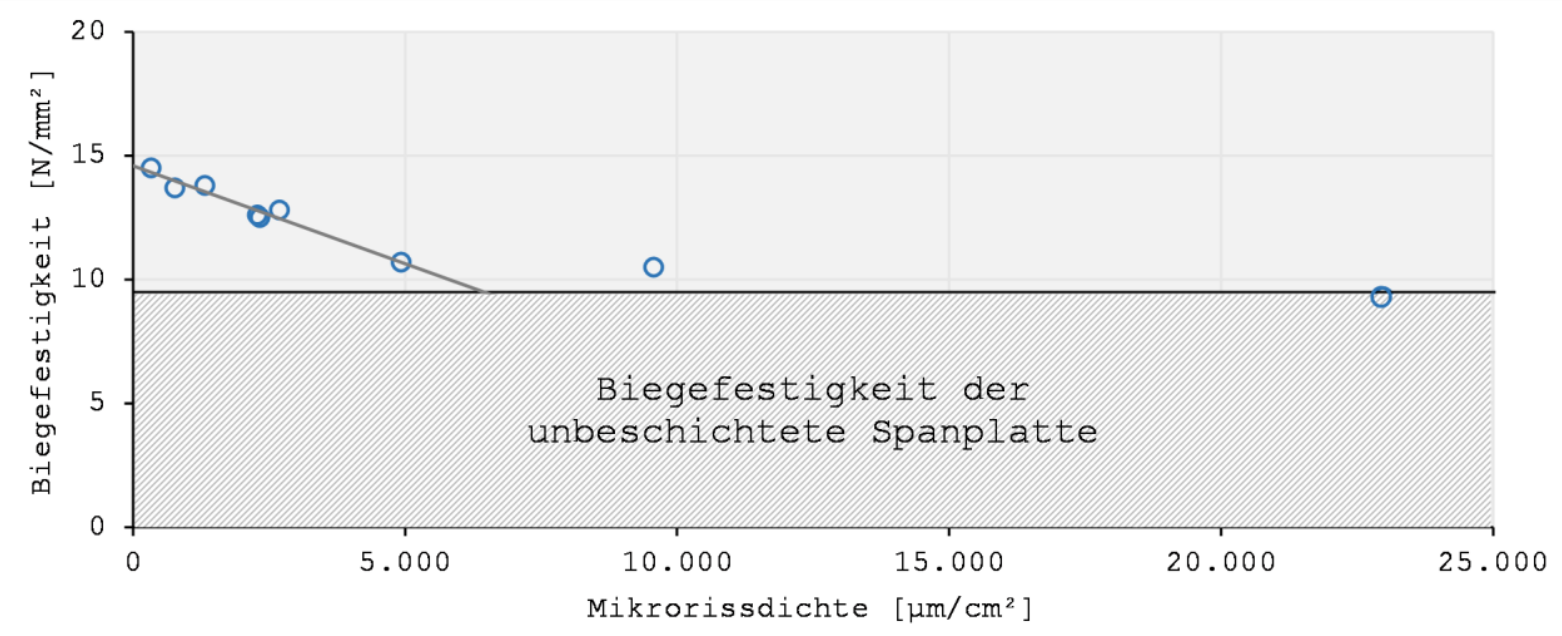

Abbildung 51: Zusammenhang von Biegefestigkeiten direktbeschichteter Platten und deren Mikrorissdichte

Auf Grund der Abwesenheit von Mikrorissen ist die Zugfestigkeit freier Filme nicht gleichzusetzen mit der Zugfestigkeit einer analog produzierten Direktbeschichtung. Viel mehr stellen freie Filme ein Festigkeitspotential dar, welches die Direktbeschichtung aufweisen könnte, würden keine Mikrorisse vorliegen.

Die Rissanfälligkeit von Direktbeschichtungen könnte möglicherweise verringert werden, indem Papiere mit höheren Zero-Span-Festigkeiten verwendet werden. Zugversuche an Papier attestieren eine geringe Festigkeit, denn es kommt nicht zu einem klassischen Versagen durch Bruch. Stattdessen 
wird der Widerstand des Papiervlieses gegen ein Auseinandergleiten der Fasern gemessen. Versagt eine Direktbeschichtung bei Zugbeanspruchung, findet ein solches Verhalten nicht statt. Es entsteht eine scharfe Bruchkante wie bei der Zero-Span-Prüfung.

Das Harz fixiert die Fasern des Papiervlieses und unterbindet ein Auseinandergleiten. Zero-SpanPrüfungen belasten Papiere daher wie sie auch als Direktbeschichtung belastet würden. Das Papier hat eine Zero-Span-Festigkeit von $41 \mathrm{~N} / \mathrm{mm}^{2}$ bei $185^{\circ} \mathrm{C}$-Proben. Die Festigkeit von vergleichbaren freien Filmen liegt bei $57 \mathrm{~N} / \mathrm{mm}^{2}$. Die Differenz kann über die Fixierung hinausgehenden Einflüssen des Harzes zugeschrieben werden. Würden Papiere mit höherer Zero-Span-Festigkeit verwendet, könnte sich die Festigkeit der Direktbeschichtung und dadurch deren Rissanfälligkeit verbessern.

\section{HYGRISCHE LÄNGENÄNDERUNGEN}

Durch eine Änderung des hygrischen Verhaltens der freien Filme oder der Spanplatte könnte sich eine Spannung in der direktbeschichteten Platte einstellen, die zur erhöhten Rissanfälligkeit führt.

Würde das hygrische Verhalten überhärteter freier Filme die erhöhte Rissanfälligkeit auslösen, müssten sie sich von weniger ausgehärteten freien Filme unterscheiden. Dies ist nicht der Fall. Ausschließen lässt sich deren Einfluss auf die Rissanfälligkeit dennoch nicht. Bei freien Filmen kommen im Gegensatz zur Direktbeschichtung keine Mikrorisse vor. Mikrorisse könnten das hygrische Verhalten verändern. Beim Quellen könnten sie sich schließen, bei Schwindungen auseinandergezogen werden. In beiden Fällen würden die hygrischen Längenänderungen sinken.

Eine Änderung im hygrischen Verhalten der Spanplatten lässt sich als Ursache für die erhöhte Rissanfälligkeit bei Überhärtung ausschließen. Es wurde nachgewiesen, dass die hygrische Längenänderungen von Spanplatten bei einer Heißverpressung unverändert bleiben. Auch eine thermische Modifizierung der äußersten Spanplattenschicht lässt sich ausschließen. Ab $150{ }^{\circ} \mathrm{C} \mathrm{kommt}$ es zu Veränderungen der Zellwandbestandteile (MAl; MILITZ 2012). Beim Direktbeschichten könnte daher die äußerste Spanplattenschicht thermisch modifiziert werden. Dies würde bei der Längenmessung nicht auffallen. Die Schieblehre liegt mittig am Stoß der Spanplatte an. Änderungen des äußersten Spanplattenbereichs werden nicht aufgenommen. Allerdings würde eine thermische Modifizierung bei der Biegeprüfung auffallen. Thermische Modifizierung führt $\mathrm{zu}$ einem Festigkeitsverlust (MAl; MILITZ 2012). Wie bereits diskutiert ist bei der Biegeprüfung die äußerste Schicht, also der vermeintlich thermisch modifizierte Bereich, ausschlaggebend für die Festigkeit. Da sich die Biegefestigkeit der Spanplatte nicht verändert, lässt sich der Einfluss einer thermischen Modifizierung ausschließen.

\section{DIFFUSIONSWIDERSTÄNDE}

Diffusionswiderstände könnten die Rissanfälligkeit beeinflussen. Zum einen können bei der Rissanfälligkeitsprüfung die geringen Diffusionswiderstände zu einer stärkeren Trocknung der Spanplatte unter der Direktbeschichtung führen. Dies kann zu einer höheren Spannung und dadurch zu vermehrter Rissbildung führen. Zum anderen könnten die Papierfasern Spannungen zum Harz aufbauen. Bei unimprägniertem Papier quellen und schwinden die einzelnen Fasern. Das damit einhergehende Abstoßen bzw. Anziehen der Fasern wird als hygrische Längenänderung des Papiers gemessen (KRASNOSHLYK 2018). Bei Direktbeschichtungen sind die Fasern in das Harz eingebettet und fixiert. Quellen die Fasern, werden Spannungen zum umgebenden Harz aufgebaut. Eine hygrische Längenänderung des gesamten freien Films könnte ausbleiben. Bei den überhärteten, diffusionsoffenen Direktbeschichtung wären diese lokalen Spannungen stärker ausgeprägt. 


\section{EIGENSPANNUNGEN}

Mit Eigenspannungen lassen sich die höheren Rissanfälligkeiten bei Überhärtung nicht erklären. Zwar zeigen die Stichmaßänderungen bei Erhöhung der relativen Luftfeuchte von 35\% auf $85 \%$ eine Abhängigkeit zu den Aushärtungsbedingungen. Diese werden jedoch bei steigender Aushärtung geringer. Um die höhere Rissanfälligkeit bei Überhärtung zu erklären, müssten sie größer werden. 


\subsection{EINFLUSSGRÖßE: BLÄTTRIGKEIT UND FÜLLGRAD DES PAPIERS}

Untersucht wurde, ob die Blättrigkeit der Direktbeschichtung und der Füllgrad des Papiers die Rissanfälligkeit der Direktbeschichtung beeinflussen. Dazu wurden 32 Platten, die sich unter anderem hinsichtlich der Blättrigkeit der Direktbeschichtung und des Papierfüllgrades unterscheiden, produziert. Als Messergebnisse sind im Folgenden die Mittelwerte der Probensätze (alle 1-blättrigen gegenüber allen 2-blättrigen Platten bzw. alle Platten mit gefüllten gegenüber allen mit ungefülltem Papier) dargestellt. Zusätzlich wurden 1-blättrige und 2-blättrige freie Filme produziert und geprüft.

\subsubsection{RISSANFÄLLIGKEIT}

Es wurde geprüft, wie ausgeprägt die Rissanfälligkeit der produzierten Platten ist. Dazu wurden Prüfkörper $(n=3)$ mit beidseitig eingebrachten Rissinitialen über 24 Stunden bei $70^{\circ} \mathrm{C}$ belastet und deren kumulierte Risslänge als Maß für die Rissanfälligkeit der Platte bestimmt.

\section{ERGEBNISSE}
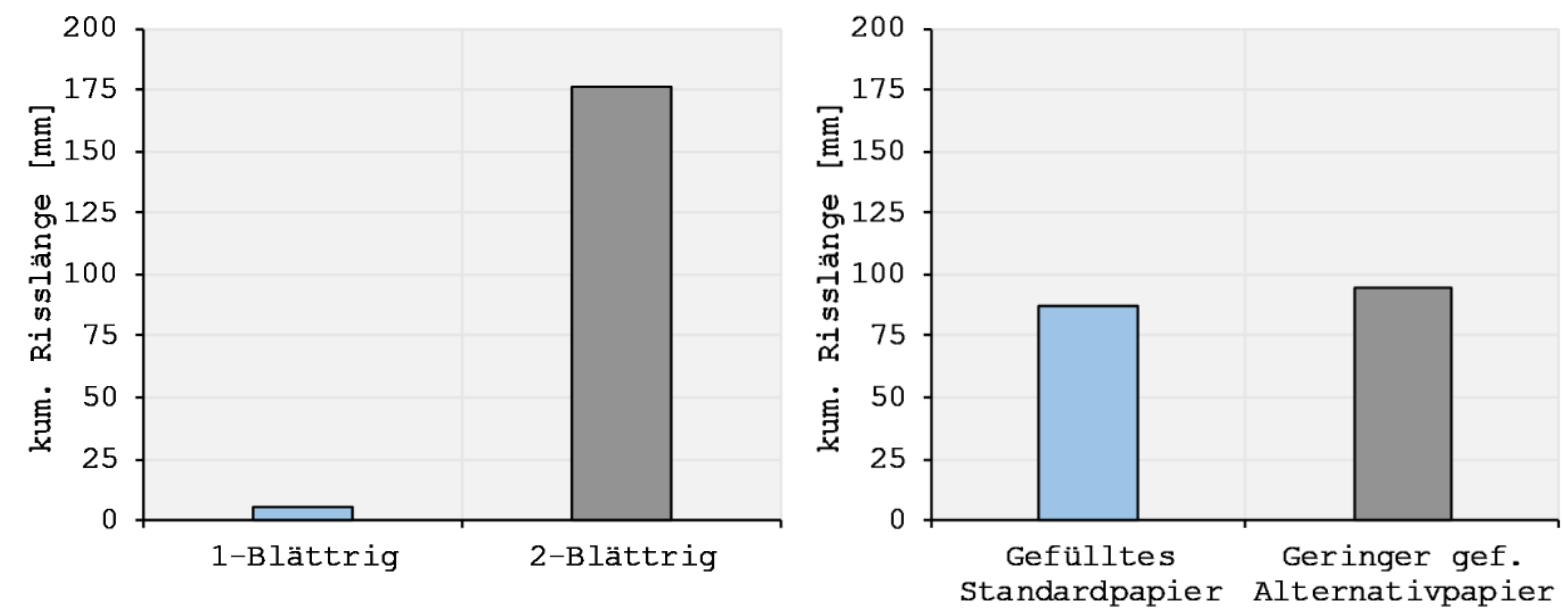

Abbildung 52: Arithmetische Mittelwerte von direktbeschichteter Platten unterschiedlicher

kumulierten Risslängen [mm] unterschiedlicher Papiere (rechts).

Abbildung 52 zeigt arithmetischen Mittelwerte. Auf Grund der hohen Vielfalt innerhalb der Probensätzen ist die Standardabweichung groß und nicht abgebildet. Dennoch kann zwischen den 1-blättrigen und 2-blättrigen Platten ein signifikanter Unterschied (99\%-Niveau) nachgewiesen werden. Die anfälligste 2-blättrige Platte hat eine kumulierte Risslänge von $600 \mathrm{~mm}$, die anfälligste 1-blättrige von $16 \mathrm{~mm}$. Die Rissanfälligkeit von Platten unterschiedlicher Papierfüllgrade unterscheidet sich nicht signifikant. 


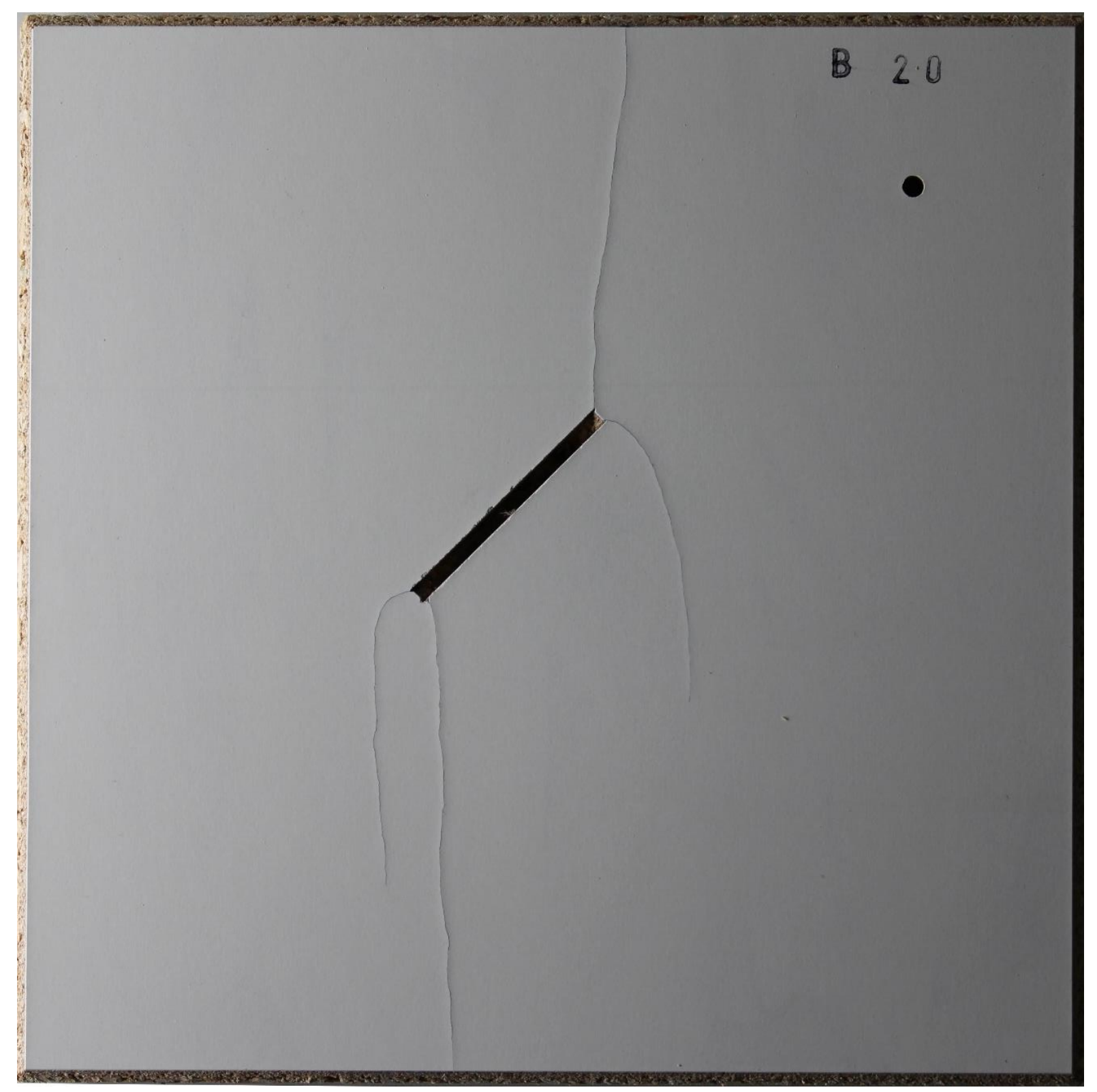

Abbildung 53: Aufnahme einer 2-Blättrigen Probe nach der Rissanfälligkeitsprüfung.

Risse bei 2-blättrigen Proben gehen stets von Nut oder Bohrloch aus. Oft verlaufen sie bis zum Probenrand. An nach links und rechts zulaufenden Ecken der Nut verlaufen die Risse zuerst waagerechten und drehen dann in die Senkrechte.

\section{DISKUSSION}

Das Rissbild 2-blättriger Platten unterscheidet sich von dem Rissbild stark überhärteter Platten (Kapitel 5.1.1). Bei 2-blättrigen Direktbeschichtungen sind die Risse stets an den Rissinitialen aufgetreten. Die Drehungen von Rissen aus der Waagerechten in die Senkrechte listet LUKOWSKY (2015) als häufiges Merkmal von Rissen auf, die an Ecken und Ausschnitten beginnen. Grund ist vermutlich eine durch die Kerbgeometrie bedingte Spannungskonzentration, wodurch der anfängliche waagerechte Rissverlauf vorgegeben wird. Mit zunehmender Entfernung von der Nutecke dominieren die anisotropen Eigenschaften des Papiers und zwingen dem Riss eine Biegung auf.

Die Rissanfälligkeit von Platten unterschiedlicher Füllgrade unterscheidet sich nicht. Industriepartnern zufolge wurde in firmeninternen Untersuchungen ein Einfluss des Füllgrades auf die Rissanfälligkeit zwar nachgewiesen: bei hohen Füllgraden kommt es zu einer Gefügeschwächung. Allerdings werden 
als Konsequenz aus dieser unveröffentlichten Studie Papiere mit einem so hohen Füllgrad nicht mehr produziert. Die Füllgrade beider Papiere liegen unter dieser Grenze.

\subsubsection{FESTIGKEITEN UND ELASTIZITÄTSMODULN}

Um zu prüfen, ob die Rissanfälligkeit 2-blättriger Direktbeschichtungen auf ein abweichendes mechanisches Verhalten zurückzuführen ist, wurden freie Filme $(n=10)$ im Zugversuch geprüft.

\section{ERGEBNISSE}
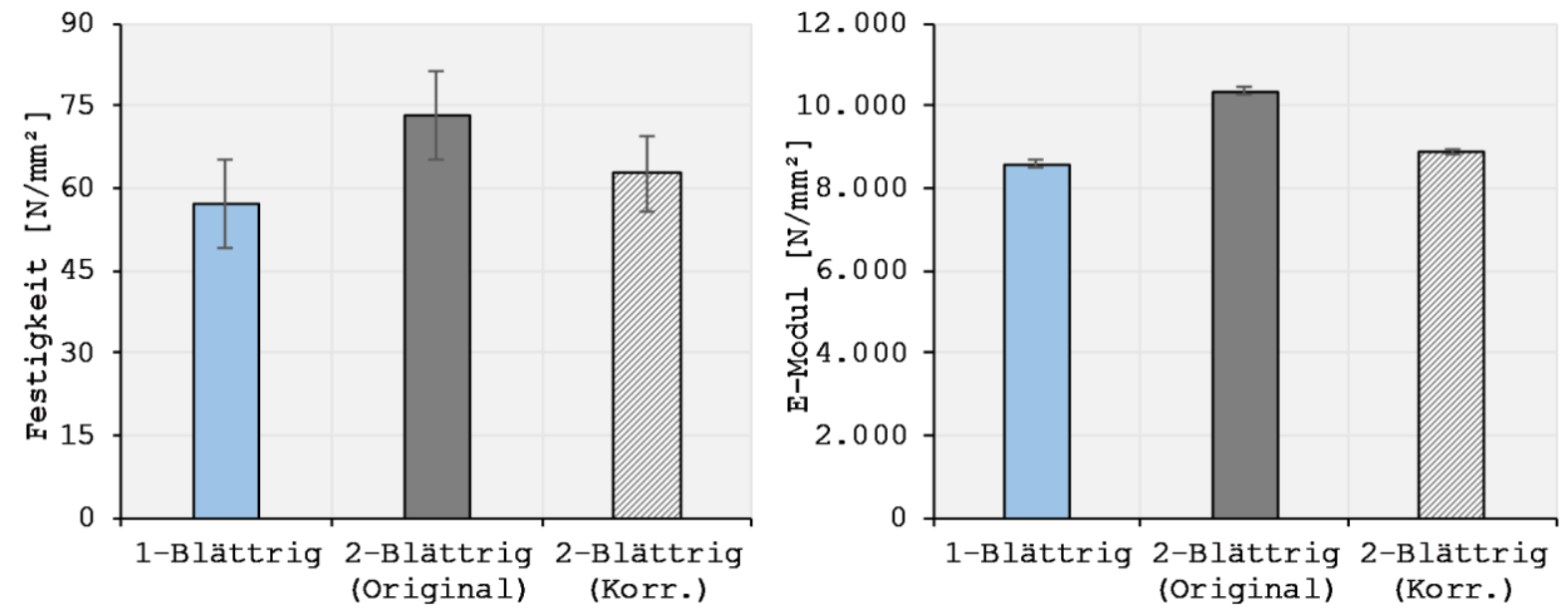

Abbildung 54: Zugfestigkeiten (links) und Elastizitätsmoduln (rechts) l-blättriger und 2-blättriger freier Filme.

Von 2-blättrigen Filmen sind die Messwerte ("Original") sowie die auf Grund der Verdichtung korrigierten Messwerte ("Korr.") abgebildet.

Die mechanischen Eigenschaften 2-blättriger freier Filme sind signifikant höher als die der 1-blättrigen (Abbildung 54). Während der Zugprüfung wurden für 1-blättrige freie Filme eine Dicke von 0,14 mm und für 2-blättrige $0,24 \mathrm{~mm}$ gemessen.

Die Prüfkörper rollen sich unterschiedlich auf: 2-blättrige freie Filme bleiben nahezu plan, 1-blättrige drehen sich auf.

\section{DISKUSSION}

Die freien Filme sind dicker als ihre Gegenstücke als Direktbeschichtung. Durch das Releasepapier kann während des Verpressens weder Harz noch durch die Polykondensation freiwerdendes Wasser in die Spanplatte eindringen. Es ist möglich, dass dadurch die freien Filme dicker sind. In Kapitel 5.2.5 werden mikroskopische Aufnahme zu den Schichtdicken dargestellt und diskutiert.

Die voneinander abweichenden mechanischen Eigenschaften 1-blättriger und 2-blättriger freier Filme lassen sich auf eine unterschiedliche Verdichtung zurückführen. Bei der Festigkeit wird die Bruchlast auf die Querschnittsfläche der Probe bezogen; auf Breite und Dicke. 2-blättrigen Filme sind nicht doppelt so dick wie 1-blättrige. Sie sind stärker verdichtet. Bezieht man die gemessene Bruchlast 2-blättriger freier Filme auf einen Querschnitt, der doppelt so groß ist wie bei 1-blättrigen, gleichen sich Elastizitätsmodule und Festigkeiten an (Abbildung 54, korrigierte Werte). Die Elastizitätsmoduln beider Gruppen sind weiterhin signifikant unterschiedlich, die Festigkeiten sind es nicht mehr.

Das unterschiedliche Aufrollverhalten der freien Filme könnte von den Produktionsbedingungen hervorgerufen werden. Das Verhältnis aus Energie pro Volumen ist bei 2-blättrigen freien Filmen geringer. Gleich eingestellte Temperatur und Druck wirken bei 2-blättrigen auf das doppelte Volumen. 
Zudem wird die Wärmeenergie zum Aushärten einseitig - also asymmetrisch - aufgebracht. Sollte das Papier einen asymmetrischen Aufbau aufweisen, könnte bei einem 2-blättrigen freien Film diese Asymmetrie verringert werden.

\subsubsection{MIKRORISSE}

Bei einer Überhärtung kommt es zu vielen Mikrorissen in der Direktbeschichtung, die eine Materialschwächung bewirken. Es wurde geprüft, ob bei den rissempfindlichen 2-blättrigen Platten ebenfalls Mikrorisse vorkommen. Dazu wurden angefärbte Mikrorisse unter einem Mikroskop vermessen und das Verhältnis aus kumulierter Risslänge zu untersuchter Fläche als Mikrorissdichte angegeben $(n=5)$.

\section{ERGEBNISSE}

Sowohl bei den 1-blättrigen als auch bei den 2-blättrigen direktbeschichteten Platten wurden keine Mikrorisse gefunden.

\section{DISKUSSION}

Die hier untersuchten Platten wurden mit kaltem Pressblech beschichtet. Bei analog produzierten Platten aus Kapitel 5.1.3 sind ebenfalls kaum Mikrorisse vorhanden. Eine Rissanfälligkeit durch Mikrorisse lässt sich daher ausschließen.

\subsubsection{HYGRISCHES VERHALTEN}

Bei 2-blättrigen Direktbeschichtungen könnte ein ausgeprägteres hygrisches Verhalten größere Spannungen zur Spanplatte aufbauen und so die Platte rissanfälliger werden. Um einen solchen Einfluss zu prüfen, wurde von freien Filmen die Längenänderung $(n=10)$ in Klimareihen sowie das Sorptionsverhalten $(n=1)$ in einem Klimazyklus bestimmt.

\section{ERGEBNISSE}
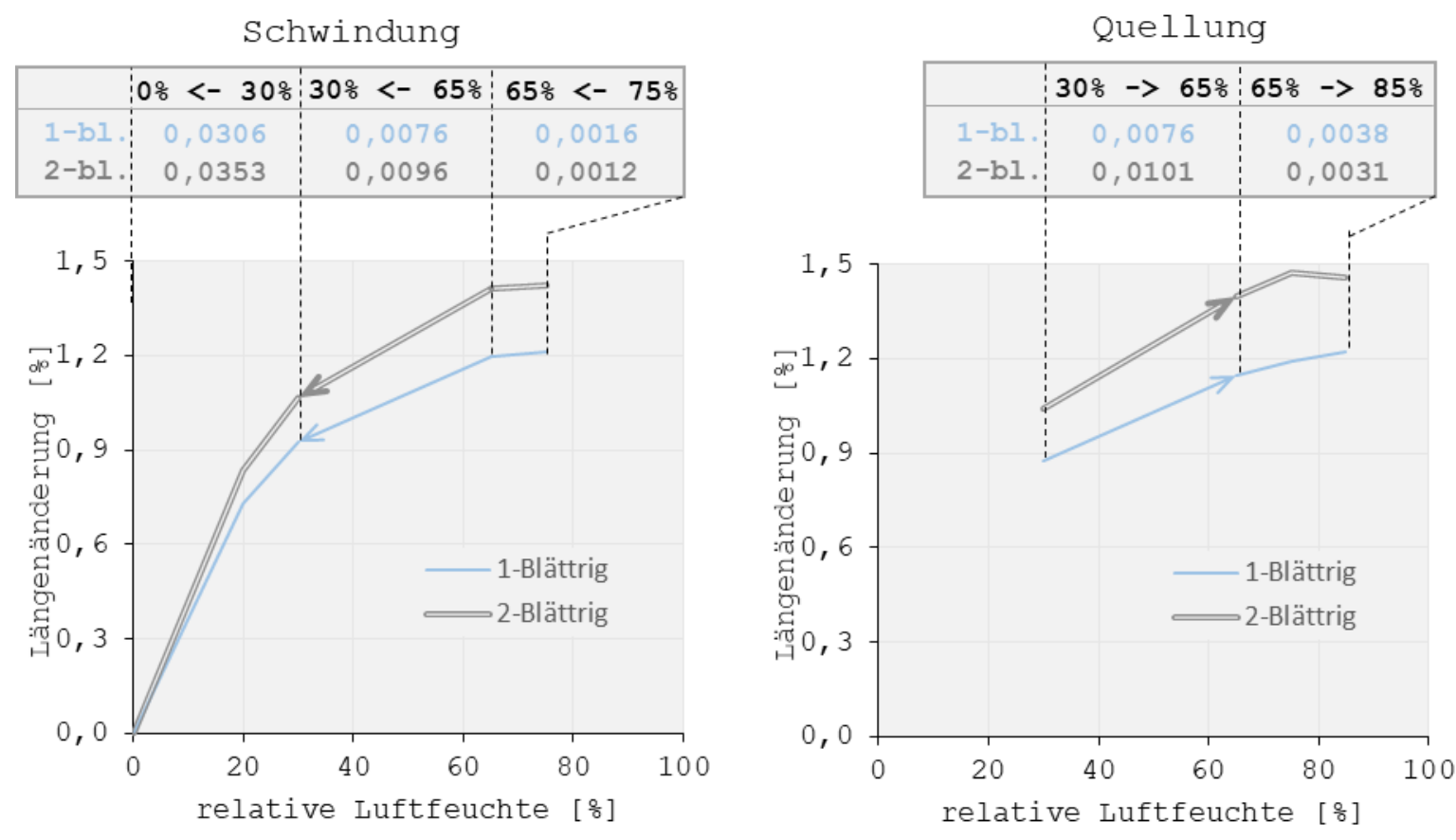

Abbildung 55: Kurven zur hygrischen Längenänderung freier Filme. 
In der tabellarischen Auflistung sind differentielle Quellmaße [응] angegeben. "1-bl." steht für 1-blättrige, "2-bl. " für 2-blättrige freie Filme.

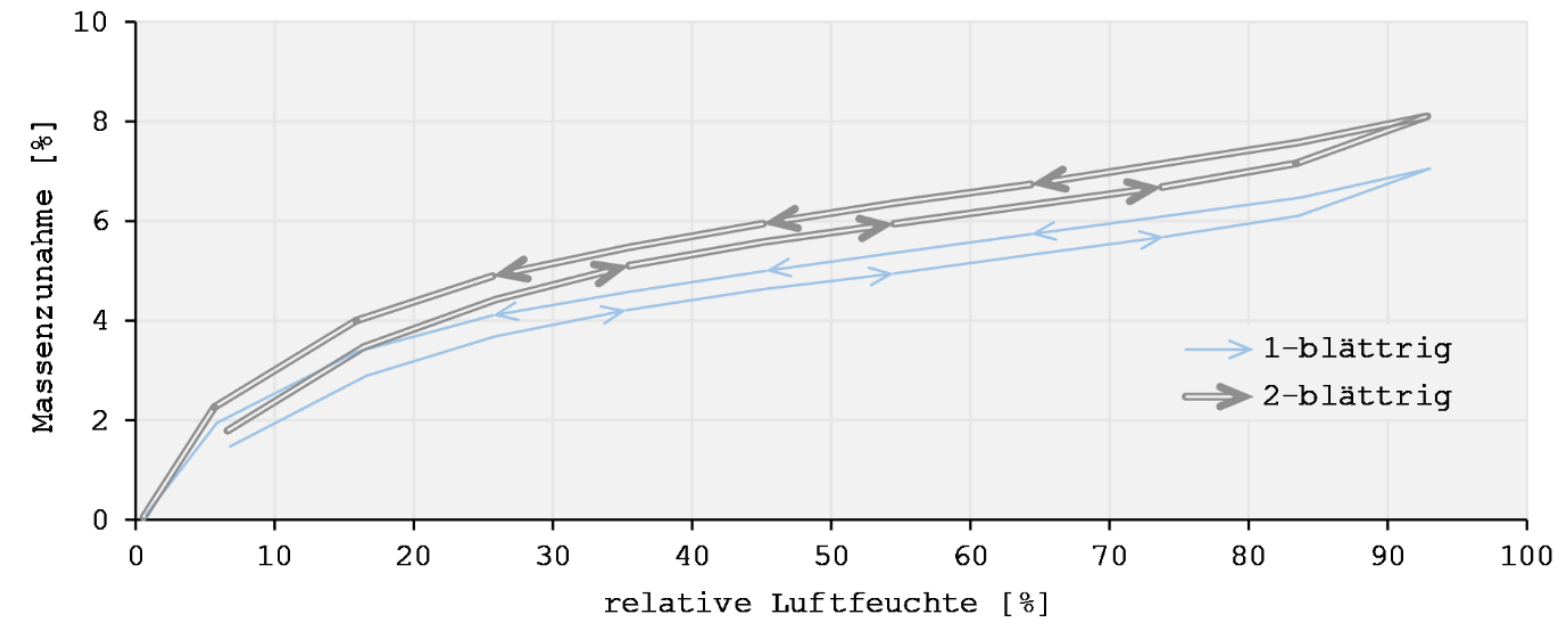

Abbildung 56: Sorption und Desorption von 1-blättrigen und 2-blättrigen freien Filmen.

Die hygrische Längenänderung als auch die relative Massenzunahme 2-blättriger Filme sind höher als bei den 1-blättrigen Gegenstücken.

\section{DISKUSSION}

Tabelle 32: Längen- und Massenänderung bei Quellung freier Filme sowie von OEHLER (2016) vermessenen Materialien.

Alle Längen beziehen sich auf den darrtrocknen Zustand.

\begin{tabular}{l|c|c|c|} 
& $30 \%$ rel. Lf. & $65 \%$ rel. Lf. & $90 \%$ rel. Lf. \\
\hline Längenänderung [\%] & 0,7 & 0,9 & 1,1 \\
- OeHLer (2016) & 0,9 & 1,2 & 1,2 \\
- Messwert 1-blättrig & 1,1 & 1,4 & 1,4 \\
- Messwert 2-blättrig & 3,5 & 5 & 7 \\
\hline Massenzunahme [\%] & 4 & 5,5 & 6,8 \\
- OeHter (2016) & 4,5 & 6,5 & 8
\end{tabular}

Die hygrischen Längenänderungen der freien Filme liegen über Werten von OEHLER (2016). OEHLER (2016) hat nur ein Dekor- und ein Kernpapier untersucht (Tabelle 32). Entsprechend können dessen Werte lediglich als grobe Richtwerte interpretiert werden.

Das unterschiedliche hygrische Verhalten von 1-blättrigen und 2-blättrigen freien Filmen ist unerwartet. Durch eine reine Verdoppelung des Volumens sollte sich das hygrische Verhalten nicht verändern. Ein Messfehler ist jedoch unwahrscheinlich: zwei voneinander unabhängige Versuche (sowohl hygrische Längenänderung als auch DVS) zeigen dieses Verhalten. Das unterschiedliche hygrische Verhalten der freien Filme könnte, analog zu deren Aufrollverhalten, den Produktionsbedingungen geschuldet sein. Der einseitige und bezogen auf das Volumen schwächere Energieeintrag bei 2-blättrigen freien Filmen könnte die abweichenden Eigenschaften hervorrufen. Ein 
damit assoziierter schwächerer Aushärtungsgrad erklärt jedoch nicht das hygrische Verhalten. Hygrisches Verhalten und Aushärtungsgrad sind nicht abhängig voneinander (Kapitel 5.1.5.1). Wodurch letztlich das abweichende hygrische Verhalten 2-blättriger freier Filme ausgelöst wird, konnte nicht geklärt werden. 


\subsubsection{MIKROSKOPIE}

Proben wurden mit einem Mikroskop untersucht.

\section{ERGEBNISSE}
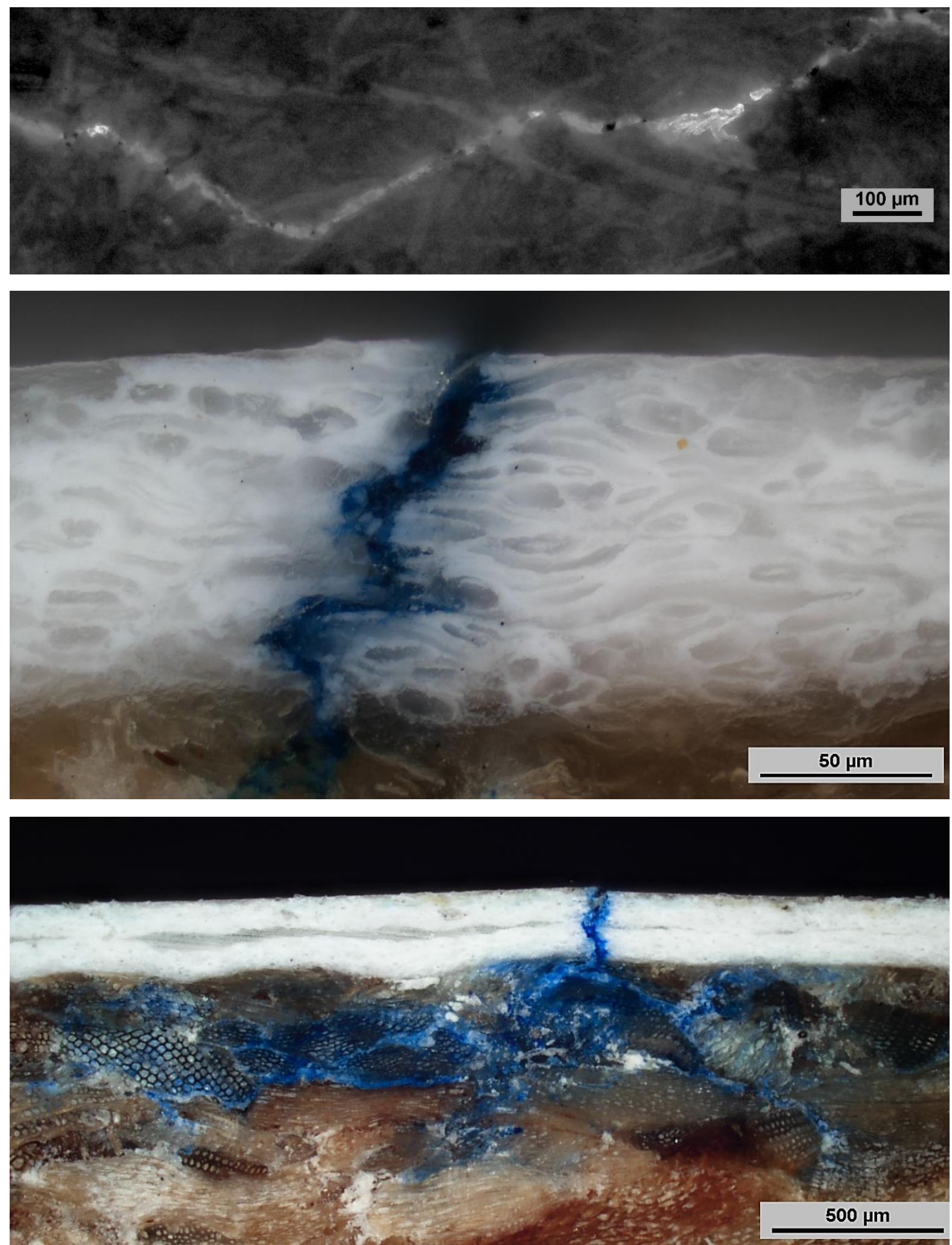

Abbildung 57: Aufsicht auf einen Riss bei einer 1-blättrigen Direktbeschichtung (oben) und Querschnitte von gerissenen 1-blättrigen (Mitte) und 2-blättrigen Proben (unten) Bei der Aufsicht ist die Bruchkante des Risses hell hervorgehoben. In blassen Grautönen ist die Fluoreszenz der Papierfasern der Direktbeschichtung abgebildet. Die Aufnahme wurde mit Bildbearbeitung optimiert, um den Kontrast der Fasern zu erhöhen. 
Bei 1-blättrigen Varianten orientieren sich die Risse an oberflächennahen Papierfasern (Abbildung 57). Ein vergleichbares Verhalten lässt sich bei 2-blättrigen Direktbeschichtung nicht beobachten. Risse bei 2-blättrigen Proben setzen sich bis zu $1 \mathrm{~mm}$ in die Spanplatte fort. Der Riss gabelt meist und setzt sich teilweise mehrere Millimeter zur Seite fort. Über den gesamten Querschnitt hat sich zwischen den Imprägnatblättern 2-blättriger Direktbeschichtung eine augenscheinlich papierfreie Zwischenschicht gebildet. Solche „Harzseen“ wurden bei sämtlichen untersuchten Direktbeschichtung als häufig vorkommende, äußerste Schicht beobachtet.
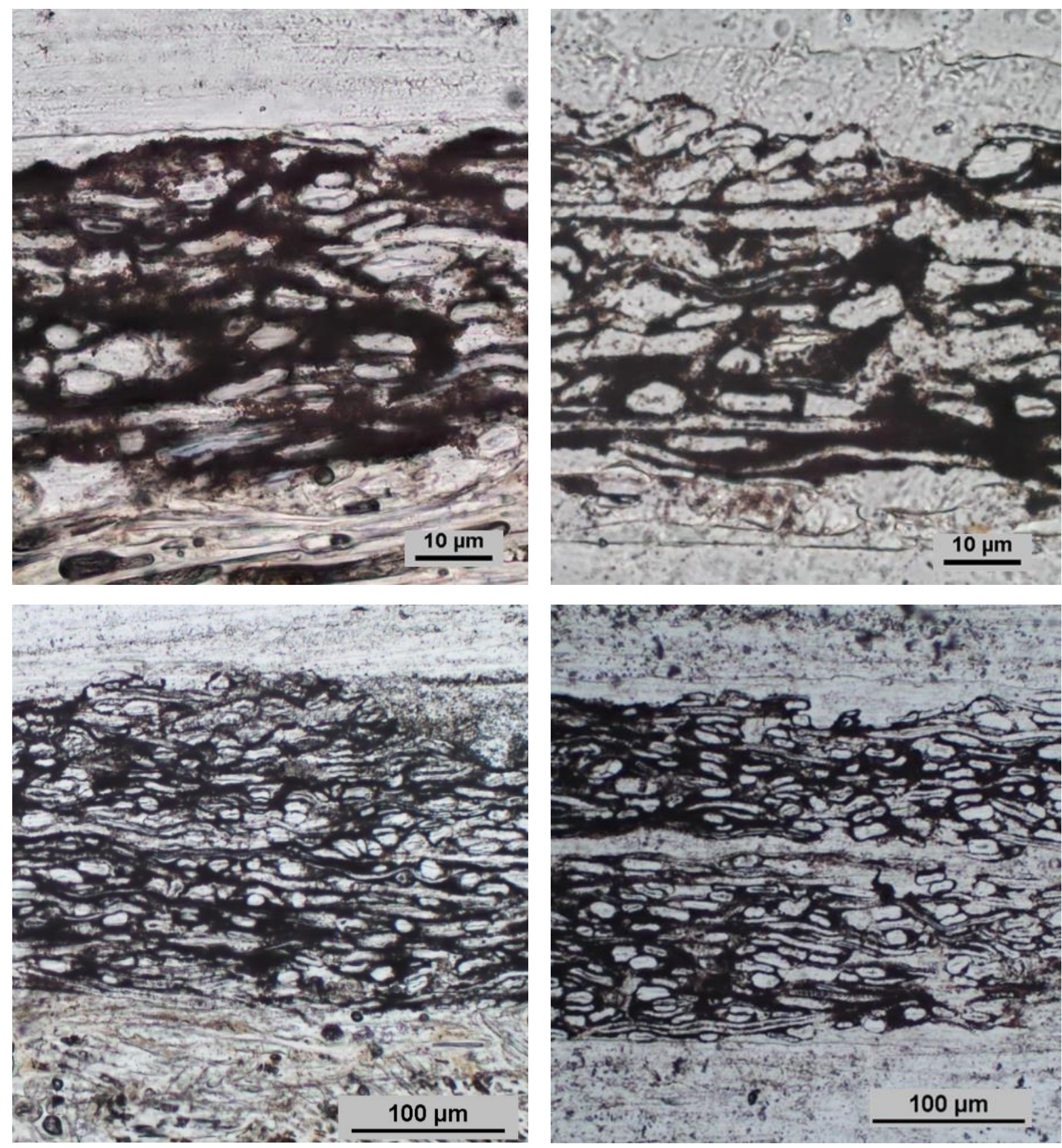

Abbildung 58: Dünnschliffe von 1-blättrigen (oben) und 2-blättrigen (unten) Direktbeschichtungen (links) und freien Filmen (rechts)

Freie Filme sind dicker als deren Gegenstücke als Direktbeschichtung (Abbildung 58). Bei 2-blättrigen Direktbeschichtungen und bei 2-blättrigen freien Filmen wurde an der oberen Schicht die gleiche Dicke 
gemessen: $100 \mu \mathrm{m}$. Die untere Schicht ist jedoch bei 2-blättrigen freien Filmen mit $130 \mu \mathrm{m}$ dicker als die der Direktbeschichtung mit $100 \mu \mathrm{m}$. Bei 1-blättrigen Varianten wurden analoge Dicken von $100 \mu \mathrm{m}$ für Direktbeschichtungen und $130 \mu \mathrm{m}$ für freie Filme gemessen. Bei Direktbeschichtungen wurden keine Harzseen (augenscheinlich papierfreie Bereiche aus reinem Harz) zwischen der Direktbeschichtung und der Spanplatte festgestellt. Solche Harzseen kommen bei den freien Filmen regelmäßig vor.

\section{DISKUSSION}

Risse über den Fasern deuten bei 1-blättrigen Platten auf einen oberflächennahen Effekt. Ein analoges Verhalten wurde bereits bei Mikrorissen erkannt (Kapitel 5.1.4). Eine Rissorientierung an oberflächennahen Fasern konnte bei 2-blättrigen Direktbeschichtungen nicht beobachtet werden. Bei 2-blättrigen liegt vermutlich eine andere Spannungsverteilung ohne hohe Spannungen in der äußersten Schicht vor.

Bei 1-blättrigen Direktbeschichtungen enden die Risse oft am oder kurz unter dem Übergang zur Spanplatte, bei 2-blättrigen setzen sich die Risse tiefer in die Spanplatte fort. Laut LUKOWSKY (2016) können Spannungen eines Laminats durch Verformungen der darunterliegenden Spanplatte abgebaut werden. Unter offenen Laminatstößen ist dies nicht möglich, die Spanplatte reißt bis in eine Tiefe, in der die Spannung erneut durch Verformung abgebaut werden kann, ein. Bei der Rissausbreitung einer Direktbeschichtung in die Spanplatte ist ein analoges Verhalten zu erwarten. Der Riss setzt sich in die Spanplatte fort, bis die Spannung zwischen Direktbeschichtung und Spanplatte durch eine Verformung aufgefangen werden kann. Demnach spricht das tiefere Eindringen der Risse bei 2-blättrigen Direktbeschichtung für eine höhere Spannung.

Zwischen den einzelnen Blättern 2-blättriger freier Filme und Direktbeschichtungen haben sich Harzseen gebildet. Am Fraunhofer WKI werden immer wieder Schadensanalysen zu Direktbeschichtungen angefertigt. Eine solche Zwischenschicht mit Harzseen, wie sie hier vorliegt, wurde nicht beobachtet. Diese Schicht könnte ein Hinweis für eine unzureichende Eignung der Imprägnate für eine 2-blättrige Direktbeschichtung sein. Bei 1-blättrigen Direktbeschichtungen gleichen Harzseen Unregelmäßigkeiten im Papier aus und bilden so eine möglichst ebene Oberfläche. Ein solches Ausgleichen von Unregelmäßigkeiten erscheint zwischen zwei Schichten nicht notwendig.

Bei den freien Filmen ist stets die Schicht, die mit dem Releasepapier Kontakt hatte, dicker als bei den Direktbeschichtungen. Diese unterschiedlichen Dicken könnten den Produktionsprozessen geschuldet sein. Bei Direktbeschichtungen könnte Harz und Kondenswasser der Vernetzung in die Spanplatte entweichen. Bei freien Filmen ist das auf Grund des Releasepapiers nicht möglich. Harz und Wasser könnten im Imprägnat verbleiben und so die höhere Dicke der freien Filme hervorrufen. 


\subsubsection{ABSCHLIEßENDE DISKUSSION ZUR VERSUCHSREIHE}

Bei der Versuchsreihe zu den Aushärtungsbedingungen wurden hohe Mikrorissdichten bei rissanfälligen Direktbeschichtung gemessen. Die Hypothese, eine erhöhte Mikrorissdichte setzt die Festigkeit herab und führt dadurch zu Rissen, ist nicht auf diese Versuchsreihe übertragbar. Die Festigkeiten der 1-blättrigen und 2-blättrigen freien Filme unterscheiden sich nicht. Auf Grund des doppelten Aufbaus 2-blättriger Direktbeschichtung halten diese sogar eine doppelte so hohe Bruchlast aus. Allerdings wurden die freien Filme mit vorgeheiztem Pressblech produziert, die direktbeschichteten Platten dieser Versuchsreihe nur mit kalten Pressblechen. Die Eigenschaften der freien Filme sind also nur bedingt auf die Direktbeschichtung übertragbar. Allerdings ist die Rissanfälligkeit von mit vorgeheiztem Pressblech produzierten Platten deutlich erhöht (Kapitel 5.1). Einflüsse auf die Rissanfälligkeit sollten verstärkt sein. Da dennoch keine unterschiedlichen mechanischen Eigenschaften gemessen wurden, lassen sich diese als Ursache für die Rissanfälligkeit 2-blättriger direktbeschichteter Platten ausschließen.

Ursächlich für die höhere Rissanfälligkeit 2-blättriger Direktbeschichtungen könnte eine höhere Spannung durch deren stärkeres Quellen und Schwinden sein. LAZARUS; PAUCHARD 2011 und KIM; NAIRN 2000 beschreiben Spannungen zwischen der Beschichtung und dem Substrat als gespeicherte potentielle Energie. Wird eine bestimmte Energieschwelle überschritten, kommt es zum Materialversagen. Diese Energieschwelle ist von der Materialdicke abhängig. Je dicker die Beschichtung, desto mehr potenzielle Energie kann aufgenommen werden, bevor es zum Materialversagen kommt. Die ausgeprägten hygrischen Längenänderungen der Direktbeschichtung könnten zu höheren Spannungen zur Spanplatte und damit zu hohen potenziellen Energien führen. 2-blättrige Direktbeschichtungen können zwar auf Grund ihrer Dicke mehr dieser potentiellen Energie aufnehmen. Allerdings möglicherweise nicht in einem Umfang, wie es die vermeintlich hohen hygrische Längenänderungen verursachen. Es kommt zur Rissbildung. Diese Hypothese wird durch die mikroskopischen Aufnahmen bekräftigt. Risse bei 2-blättrigen Direktbeschichtungen ragen tiefer in die Spanplatte. Das kann durch die höhere freiwerdende Energie erklärt werden. 


\subsection{EINFLUSSGRÖßE: MATERIALFEUCHTE}

In Abhängigkeit von Lagerungsbedingungen kann die Materialfeuchte der Spanplatte und des Imprägnats variieren. Es wurden solche Lagerungsbedingungen nachgestellt, Spanplatten und Imprägnate mit unterschiedlichen Feuchten verpresst und die Platten hinsichtlich der Rissanfälligkeit geprüft.

\subsubsection{RISSANFÄLLIGKEIT}

Es wurde geprüft, wie ausgeprägt die Rissanfälligkeit der produzierten Platten ist. Dazu wurden Prüfkörper $(n=3)$ mit beidseitig eingebrachten Rissinitialen über 24 Stunden bei $70^{\circ} \mathrm{C}$ belastet und deren kumulierte Risslänge als Maß für die Rissanfälligkeit der Platte bestimmt.

\section{ERGEBNISSE}

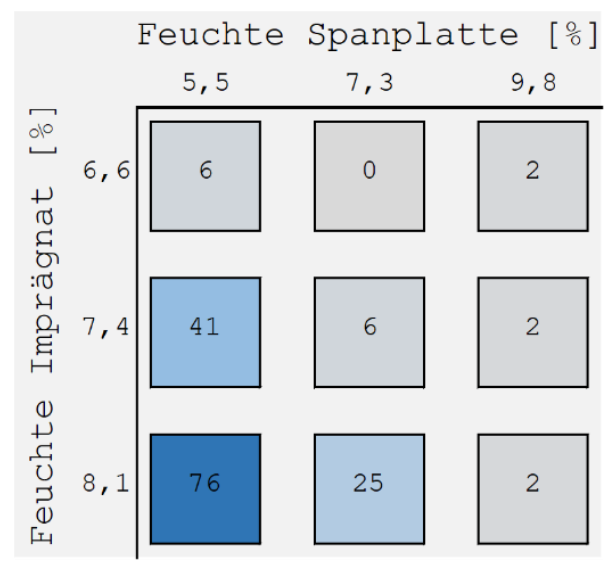

$\begin{array}{llll}\text { Abbildung 59: } & \text { Kumulierte Risslängen [mm] direktbeschichteter } & \text { Platten } \\ \text { unterschiedlicher Materialfeuchten beim Verpressen. } & \end{array}$

Mit zunehmender Feuchte des Imprägnats und abnehmender Feuchte der Spanplatte steigt die Rissanfälligkeit der Platte (Abbildung 59).

\section{DISKUSSION}

Die erhöhte Rissanfälligkeit bei bestimmten Feuchtekonstellationen lässt sich auf Spannungen zurückführen. Akklimatisieren sich die rissanfälligen Platten nach der Produktion, quillt die trockene Spanplatte und schwindet die feuchte Direktbeschichtung. Durch die gegenläufigen Dimensionsänderungen werden Spannungen zwischen den Schichten induziert.

Bei anderen Versuchsreihen wurden keine Materialfeuchten bestimmt. Es wird vermutet, dass die Platte aus trockenem Imprägnat und trockener Spanplatte den unklimatisierten Platten der anderen Versuchsreihen am nächsten kommt. 


\subsubsection{MIKRORISSE}

Bei einer Überhärtung kommt es zu vielen Mikrorissen in der Direktbeschichtung, die eine Materialschwächung bewirken. Es wurde geprüft, ob die Mikrorissdichte auch bei unterschiedlichen Feuchtekonstellationen die Rissanfälligkeit beeinflusst. Dazu wurden angefärbte Mikrorisse unter einem Mikroskop vermessen und das Verhältnis aus kumulierter Risslänge zu untersuchter Fläche als Mikrorissdichte angegeben $(n=5)$.

\section{ERGEBNISSE}

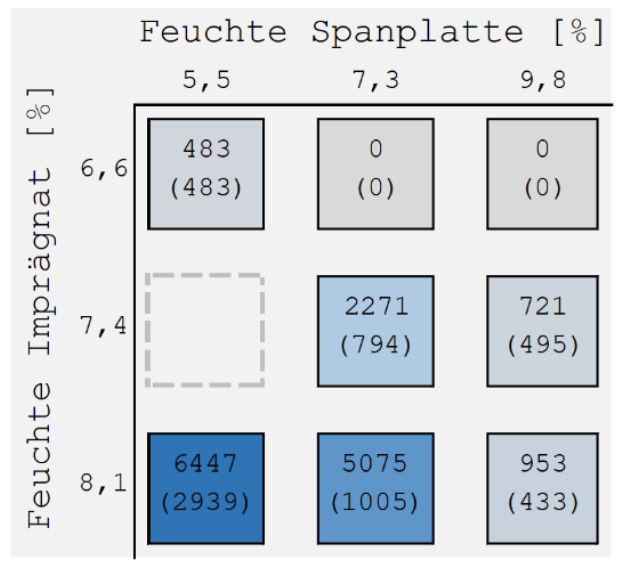

Abbildung 60: Mikrorisse [ $\left.\mathrm{\mu m} / \mathrm{cm}^{2}\right]$ direktbeschichteter Platten unterschiedlicher Materialfeuchten beim Verpressen.

In den Klammern stehen die Standardabweichungen.

Je feuchter das Imprägnat und je trockner die Spanplatte, desto höher ist die Mikrorissdichte der Platten.

\section{DISKUSSION}

Die Mikrorissdichten zeigen einen anlogen Gradienten, wie die Rissanfälligkeiten. Wie bereits diskutiert kommt es auf Grund von gegenläufigen Dimensionsänderungen zu Spannungen zwischen der Direktbeschichtung und der Spanplatte. Diese Spannungen können die Bildung von Mikrorissen verursachen. 


\subsubsection{EIGENSPANNUNGEN}

Es sollte geprüft werden, ob die zuvor beschriebenen Spannungen durch gegenläufige Dimensionsänderungen nachgewiesen werden können. Dazu wurden Proben $(n=5)$ bestehend aus Spanplattendeckschicht und Direktbeschichtung erst bei 30\% rel. Lf., dann $85 \%$ rel. Lf. klimatisiert und das Stichmaß der verformten Proben als Maß für deren Eigenspannung vermessen.

\section{ERGEBNISSE}

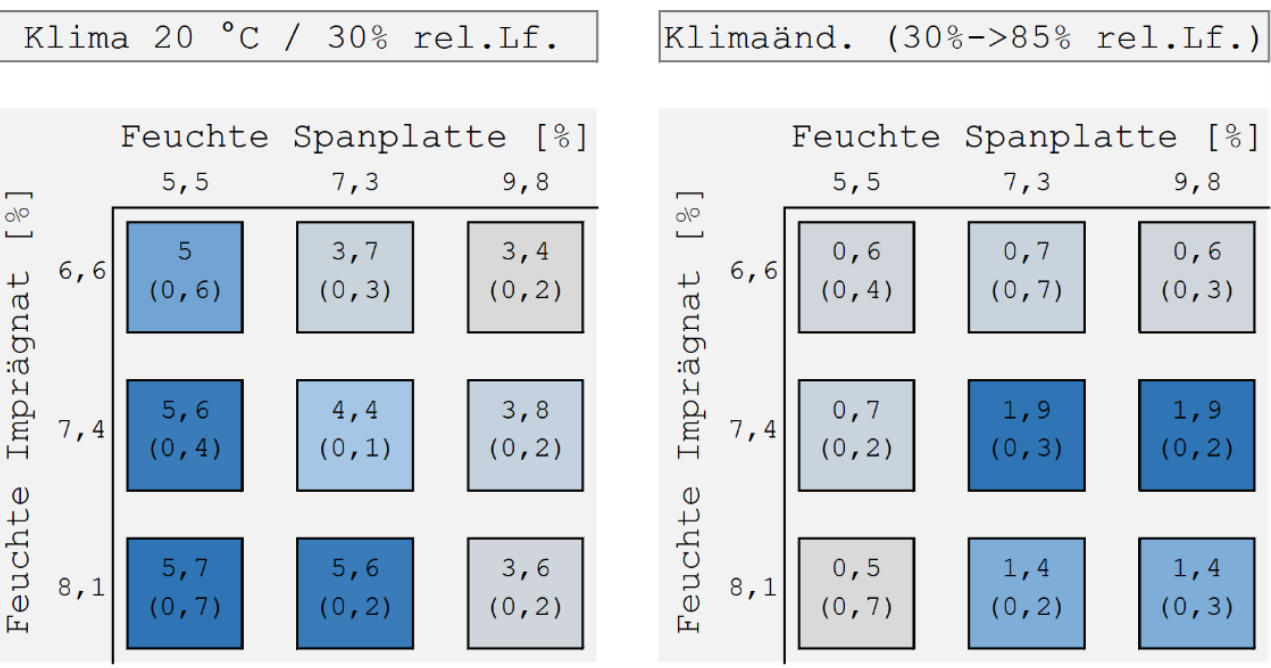

Abbildung 61: Stichmaß [mm] beim ariden Klima (links) und Änderungen des Stichmaßes [mm], wenn die relative Luftfeuchte von 30\% auf 85\% erhöht wird (rechts).

In den Klammern stehen die Standardabweichungen.

Sowohl bei $30 \%$ rel. Lf. (Abbildung 61 ) als auch bei $85 \%$ rel. Lf. (nicht abgebildet) weisen die Platten den gleichen Gradienten bei den Stichmaßen auf. Je feuchter das Imprägnat und je trockner die Spanplatte beim Heißverpressen, desto höher ist das Stichmaß der Platte. Die Stichmaßänderungen bei Erhöhung der relativen Luftfeuchte zeigen keinen Trend.

\section{DISKUSSION}

Wie bereits diskutiert treten beim Akklimatisieren der Platten gegenläufige Dimensionsänderungen auf. Es werden Spannungen zwischen der Direktbeschichtung und der Spanplatte induziert. Der Stichmaßgradient bei $30 \%$ rel. Lf. bestätigt diese Hypothese. Platten, bei denen dieser Effekt stark ausgeprägt sein sollte, zeigen hohe Eigenspannungen.

\subsubsection{ABSCHLIEßENDE DISKUSSION ZUR VERSUCHSREIHE}

Durch die gegenläufigen Dimensionsänderungen, wenn gequollene Imprägnate auf geschwundene Spanplatten verpresst werden, entstehen hohe Spannungen. Diese konnten mit den Stichmaßen identifiziert werden. Die Spannungen führen zu Mikrorissen, welche wiederum die Direktbeschichtung schwächen. Entsprechend lässt sich die erhöhte Rissanfälligkeit bestimmter Feuchtekonstellationen mit der Mikrorisshypothese aus Kapitel 5.1.9 erklären. Demnach führen Mikrorisse zu einer Festigkeitsabnahme. Platten mit einer hohen Mikrorissdichte reißen deshalb bei einer Belastung wie bei der Rissanfälligkeitsprüfung stärker. 


\subsection{EINFLUSSGRÖßE: OBERFLÄCHENDEFEKTE}

Aus Schadensanalysen zu Direktbeschichtung ist bekannt, dass Defekte in der Spanplatte zu Rissen in der darüber liegenden Direktbeschichtung führen können. Um diesen Einfluss zu untersuchen, wurden verschiedenste Defekte in die Spanplattenoberfläche eingebracht und die Spanplatte direktbeschichtet.

\subsubsection{RISSANFÄLLIGKEIT (MODIFIZIERTE VARIANTE)}

Es wurde geprüft, wie ausgeprägt die Rissanfälligkeit der produzierten Platten ist. Dazu wurden Prüfkörper ( $n=2)$ über 24 Stunden bei $70^{\circ} \mathrm{C}$ belastet und deren kumulierte Risslänge als Maß für die Rissanfälligkeit der Platte bestimmt. Abweichend zur beschriebenen Methode wurden keine Rissinitialen in die Proben gefräst und es wurden die kumulierten Risslängen für $5 \mathrm{~cm} \times 5 \mathrm{~cm}$ Rasterfelder statt für die gesamte Probe bestimmt.

\section{ERGEBNISSE}

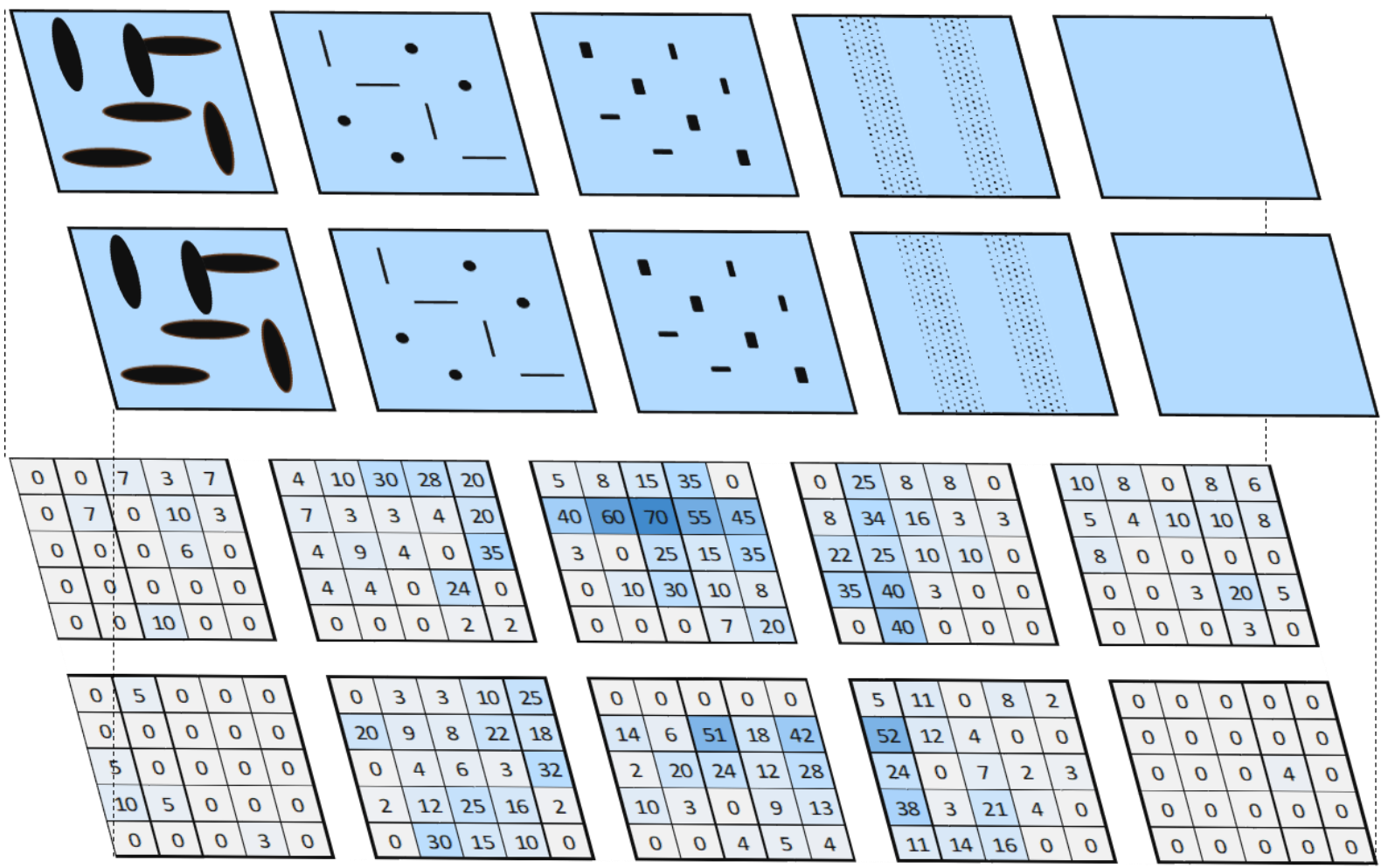

Abbildung 62: Position der Defektstellen (oben) und kumulierte Risslängen für $5 \mathrm{~cm} \times 5 \mathrm{~cm}$ Raster (unten).

Dargestellt sind die $45 \mathrm{~s}$ lang verpressten Direktbeschichtungen.

Links: Abgeschliffene Areale

Zweites von links: Bohrlöcher und Schnitte

Mitte: Fräsungen

Zweites von rechts: Entfernte einzelne Deckschichtspäne

Rechts: Unbearbeitete Referenz

Alle Defektstellen wurden auf derselben Spanplatte eingebracht. Aus der direktbeschichteten Platte wurden anschließend die Proben für die Rissanfälligkeitsprüfung gesägt. Abbildung 62 zeigt die Lage der Proben in der Spanplatte als auch kumulierte Risslängen für $5 \mathrm{~cm} \times 5 \mathrm{~cm}$ Rasterfelder.

Werden die Spanplatten $35 \mathrm{~s}$ lang beschichtet, zeigt sich nur eine marginale Rissanfälligkeit (nicht dargestellt). Die 45 s lang verpressten Direktbeschichtungen zeigen höhere kumulierte Risslängen. Bei allen Probe liegen die Risse stets abseits der Defektstellen. 


\section{DISKUSSION}

Typischerweise treten bei der Rissanfälligkeitsprüfung Risse an Nut und Bohrloch auf. Bei den hier geprüften Proben wurde auf diese Oberflächenbearbeitungen verzichtet. Die Defektstellen der Spanplatte sollten als Rissinitialen fungieren. Allerdings wurden keine Risse an diesen Defektstellen beobachtet.

Erst bei einer leichten Überhärtung bei 45 s Pressdauer entstehen Risse. Die überhärteten Platten dieser Versuchsreihe zeigen höhere kumulierte Risslängen als Proben der Versuchsreihe zu Aushärtungsbedingungen (Kapitel 5.1.1), obwohl keine Nuten oder Bohrlöcher als Rissinitialen eingebracht wurden. Das Einbringen von Defekten in die Spanplatte führt augenscheinlich zu einer Erhöhung der Rissanfälligkeit. Allerdings zeigt die Rasterdarstellung der kumulierten Risslängen einen Gradienten auf. In der Mitte der Spanplatte kommen höhere kumulierte Risslänge vor als an den Randbereichen. Dies kann auf eine Erhöhung der Rissanfälligkeit durch produktionstechnische Gründe hinweisen.

\subsubsection{TemperaturVerteILUNG IN DER PRESSE}

Der bei der Rissanfälligkeitsprüfung entdeckte Gradient könnte bei der Verpressung entstanden sein. Um diese Hypothese zu prüfen, wurden die Pressbleche bei einer späteren Verpressung mit einer Thermografie-Kamera untersucht.

\section{ERGEBNISSE}

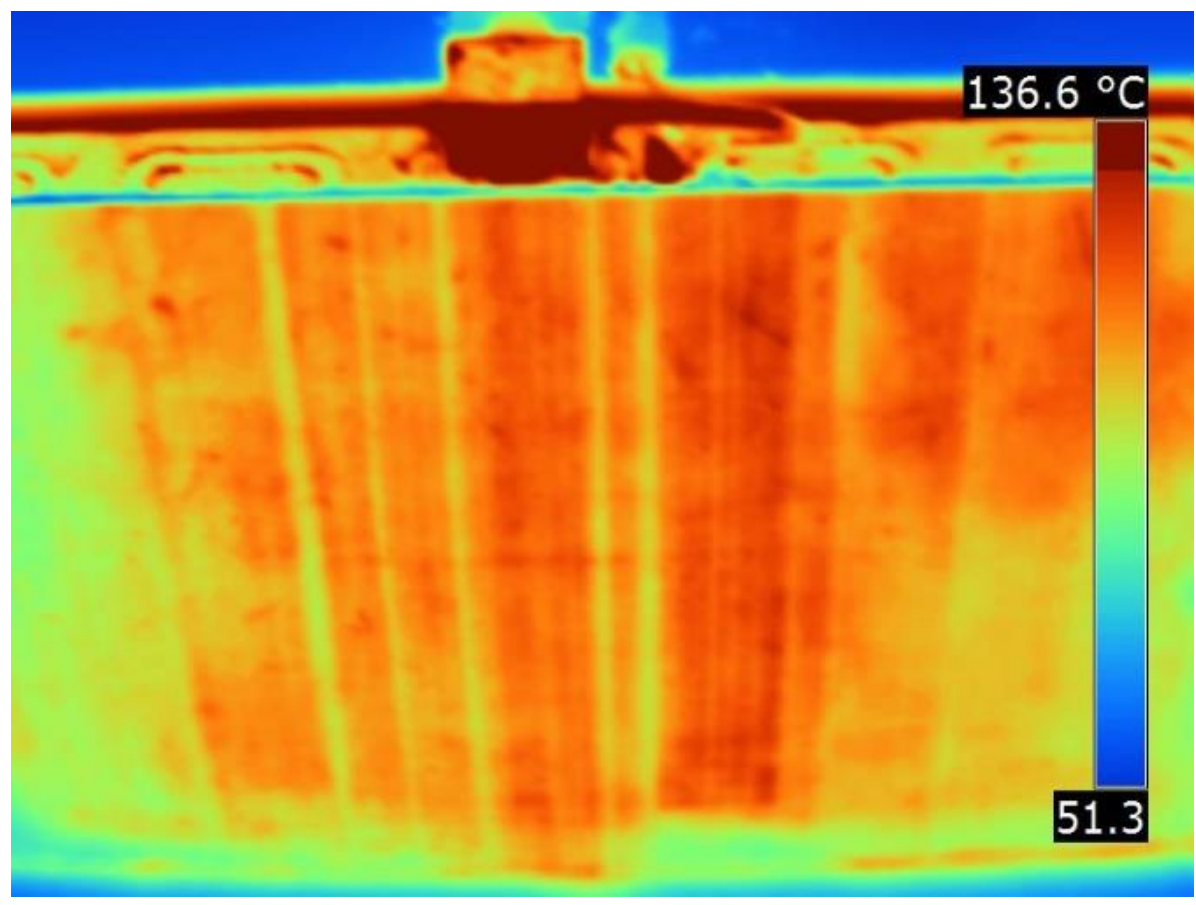

Abbildung 63: Thermografie-Aufnahmen des oberen Pressblechs direkt nach dem Direktbeschichten einer Platte.

Um das Pressblech in der Taktpresse vorzuheizen wird zuerst eine Dummy-Spanplatte verpresst. Mit dem so vorgeheizten Pressblech wird dann die Direktbeschichtung auf die gewünschte Spanplatte heißverpresst. Abbildung 63 zeigt die Oberflächentemperatur des Pressblechs nach der DummyVerpressung und vor der Verpressung der Direktbeschichtung. In der Mitte der Aufnahme hat das 
Pressblech eine Temperatur von ca. $120^{\circ} \mathrm{C}$. Zu den Seiten fällt die Temperatur des Pressblechs auf ca. $100{ }^{\circ} \mathrm{C}$ ab.

\section{DISKUSSION}

Thermografie-Aufnahmen von Stahl sind äußerst schwer zu interpretieren. Auf Grund der hohen Reflektion von Stahl wird auch die gespiegelte Fläche mitgemessen. Bei polierten Metallen ist eine thermografische Messung sogar nicht mehr möglich (GAUSSORGUES 1994). Im Falle von Abbildung 63 könnte die Reflektion des hölzernen Bodens der Technikumshalle die Aufnahme beeinflusst haben. Zudem sind Thermografieaufnahmen anfällig gegenüber Emissionen. Die dargestellten Temperaturen an den Randbereichen von Abbildung 63 können Überlagerungen der kälteren Seitenflächen der Presse sein. Als solitärer Nachweis eines Gradienten wäre diese Aufnahme auf Grund der vielen Störgrößen nicht zulässig. Da allerdings die Ergebnisse unterschiedlicher Methoden einen solchen Gradienten vermuten lassen, bestärken die Thermografieergebnisse diese Hypothese.

Wie in den Untersuchungen zu Aushärtungsbedingungen dargestellt, kann eine Erhöhung der Presstemperatur um $10^{\circ} \mathrm{C}$ zu einem deutlichen Anstieg des Aushärtungsgrades und der Rissanfälligkeit führen. Allerdings ist aufgrund der genannten Störgrößen die dargestellte Temperaturdifferenz des Pressblechs von ca. $20^{\circ} \mathrm{C}$ mit Vorsicht zu interpretieren.

\subsubsection{AUSHÄRTUNGSGRAD}

Es wurde geprüft, ob der vermeintliche Gradient bei den Aushärtungsgraden erkennbar ist. Dazu wurde von der Beschichtung geschliffenes Pulver $(n=5)$ mit Salzsäure hydrolysiert und die Menge hydrolysierten Melamins mittels UV-Spektrometer bestimmt.

\section{ERGEBNISSE}

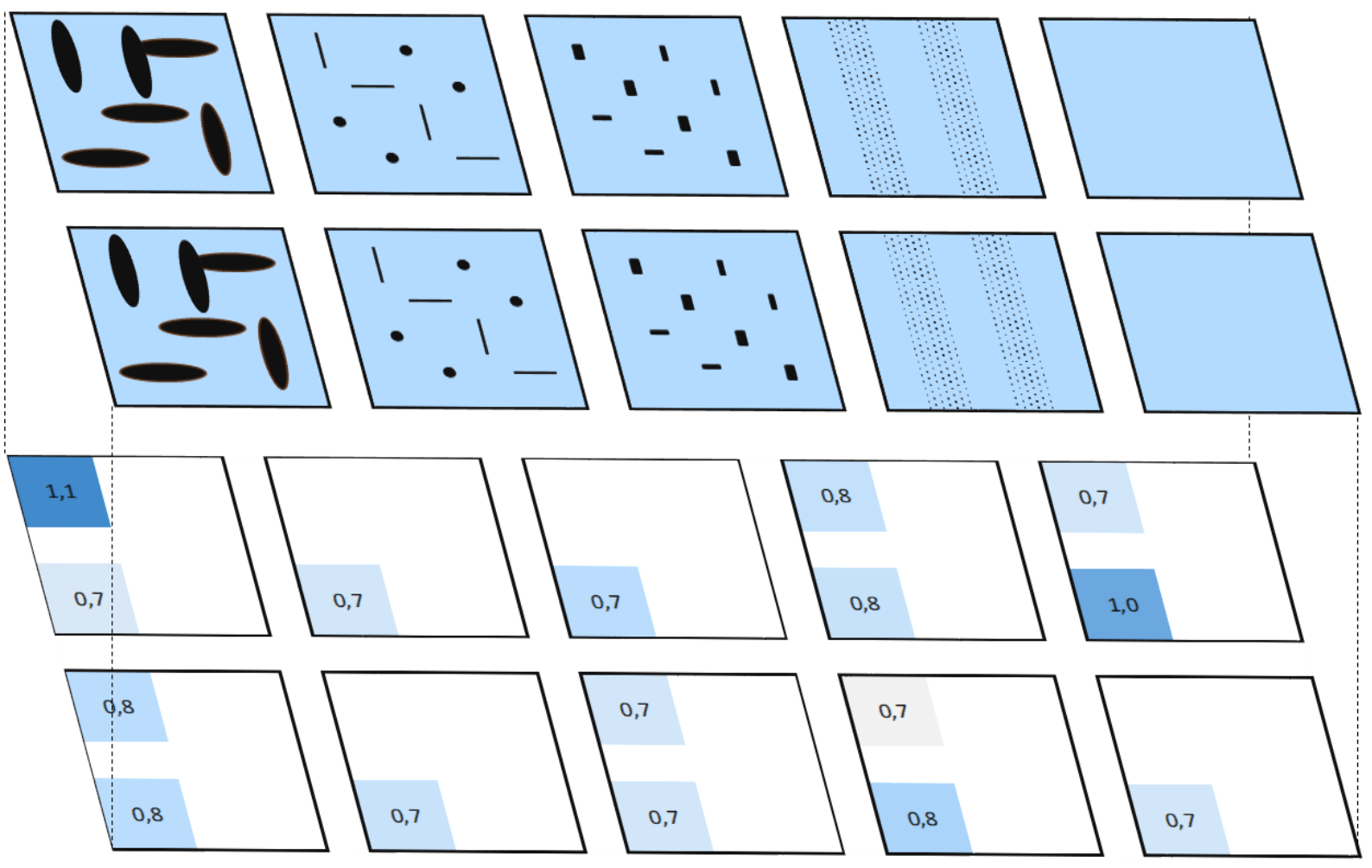

Abbildung 64: Position der Defektstellen (oben) und Aushärtungsgrade [mg/l] an ausgewählten Stellen (unten). 
Abbildung 64 zeigt, an welchen Stellen der Aushärtungsgrad bestimmt wurde und welche Melaminkonzentrationen an diesen Stellen hydrolysiert wurde. Die Mengen schwanken leicht. An den Rändern wurden vereinzelt höhere Konzentrationen gemessen.

\section{DISKUSSION}

Durch einen Vergleich zu Messwerten aus Kapitel 5.1.8 kann die untersuchte Direktbeschichtung als optimal gehärtet bis überhärtet interpretiert werden. Allerdings kann mit dieser Messmethode eine leichte nicht von einer starken Überhärtung unterschieden werden. Ein Gradient des Aushärtungsgrades kann daher weder bestätigt noch verneint werden.

\subsubsection{MIKROSKOPIE}

Die Direktbeschichtung wurde mittels Mikroskops untersucht.

\section{ERGEBNISSE}
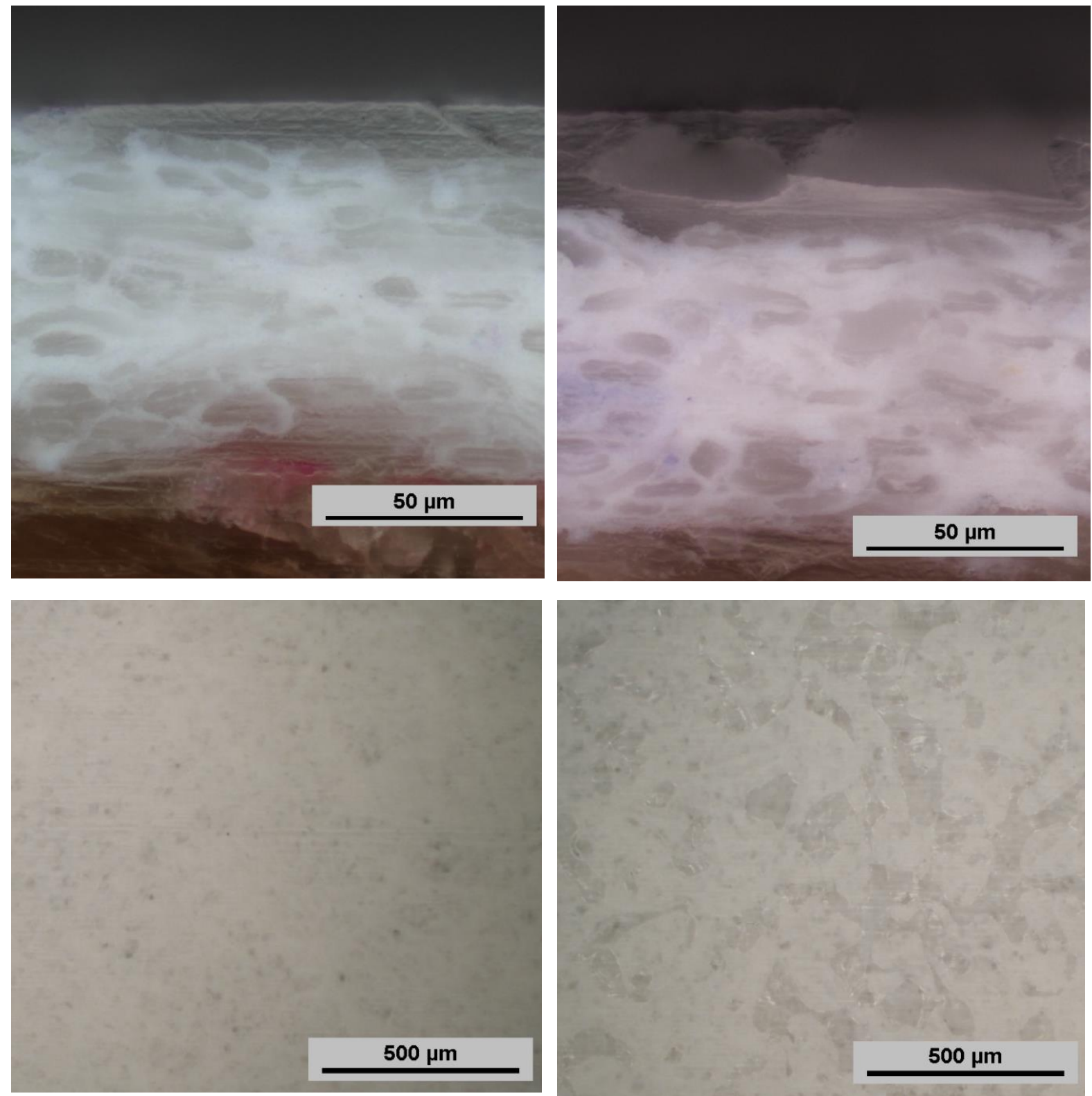

Abbildung 65: Querschnitte

(oben) und Aufsichten

(unten)

über Nuten

(rechts) und abseits von Nuten (links). 
Über Nuten und den abgeschliffenen Bereichen ist die Direktbeschichtung fleckig (Abbildung 65). Die Querschnitte an diesen Stellen zeigen Poren in der Direktbeschichtung.

\section{DISKUSSION}

Durch den Abtrag der Spanplatte an diesen Stellen liegen Täler vor. Dadurch ist der Pressdruck dort geringer und Luft könnte eingeschlossen werden. Die Direktbeschichtung zeigt über Nuten und abgeschliffenen Bereichen Luftporen. Diese liegen über der Papierbahn und sind von Harz umgeben. Diese Poren könnten von der eingeschlossenen Luft während der Verpressung hervorgerufen werden.

\subsubsection{ABSCHLIEßENDE DISKUSSION ZUR VERSUCHSREIHE}

Die kumulierten Risslängen zeigen einen Gradienten, der unabhängig von den Defektstellen in der Spanplatte zu sein scheint. Proben, die aus der Mitte der beschichteten Spanplatte entnommen wurden, haben die höchste kumulierte Risslänge. Je weiter außen die Proben lagen, desto geringer ist deren kumulierte Risslänge. Die Thermografie-Aufnahme zeigt einen Temperaturgradienten. Es wurde bereits in Kapitel 5.1.8 gezeigt, dass die Presstemperatur den Aushärtungsgrad und damit die Rissanfälligkeit beeinflusst. Entsprechend kann der Rissanfälligkeitsgradient einer Überhärtung geschuldet sein. Dafür spricht das Rissbild mit vielen kurzen Rissen. Dieses gleicht dem Rissbild von überhärteten Proben aus Kapitel 5.1.1. Mit den Aushärtungsgraden lässt sich der Gradient weder nachweisen noch widerlegen. ${ }^{3}$

Unabhängig vom Rissanfälligkeitsgradienten ist die Lage der Risse unerwartet. Zu erwarten waren Risse über den Defektstellen, wie es in Schadensanalysen bereits dokumentiert wurde. Über Defektstellen sollte die Direktbeschichtung Spannungen schlechter in den Untergrund ableiten können. Die in die Spanplatte eingebrachte Schnitte sollten analog zu Schälrissen in Sperrhölzern (Kapitel 5.5.4) eine Sollbruchstelle darstellen. Eine Unterhärtung der Direktbeschichtung über den Defektstellen könnte das dortige Fehlen von Rissen erklären. Durch die Vertiefung der Spanplatte an den größeren Defektstellen könnte sich ein Luftkissen über dem Imprägnat bilden, welches wärmedämmend wirkt. Die Direktbeschichtung über den Defekten würde schwächer aushärten. Allerdings könnte sich das Luftkissen auch zwischen dem Imprägnat und der Spanplatte bilden. In dem Fall würde die Wärme des Pressbleches vom Imprägnat aufgenommen und könnte nicht an die Spanplatte weitergegeben werden. Die Direktbeschichtung wäre überhärtet und sollte gerade in dem Fall einreißen. Möglich ist auch, dass die Defekte wie Kerben wirken. Dann würden sie Spannungen um sich herumleiten.

Wodurch die Lage der Risse abseits der Defekte hervorgerufen wird, konnte nicht abschließend geklärt werden.

3 Sollte ein produktionsbedingter Gradient vorliegen, wäre dieser auch bei den anderen Versuchsreihen vorhanden. Bei diesen wurden zu vergleichende Proben stets aus gleichen Plattenpositionen entnommen. Der vermeintliche Gradient hat daher bei anderen Versuchsreihen keinen Einfluss. 


\subsection{EINFLUSSGRÖßE: TRÄGERPLATTEN}

Untersucht wurde, ob die Trägerplatten die Rissanfälligkeit der Direktbeschichtung beeinflussen. Dazu wurden diverse Holzwerkstoffe direktbeschichtet und geprüft.

\subsubsection{RISSANFÄLLIGKEIT}

Es wurde geprüft, wie ausgeprägt die Rissanfälligkeit der produzierten Platten ist. Dazu wurden Prüfkörper ( $n=3)$ mit beidseitig eingebrachten Rissinitialen über 24 Stunden bei $70^{\circ} \mathrm{C}$ belastet und deren kumulierte Risslänge als Maß für die Rissanfälligkeit der Platte bestimmt.

\section{ERGEBNISSE}

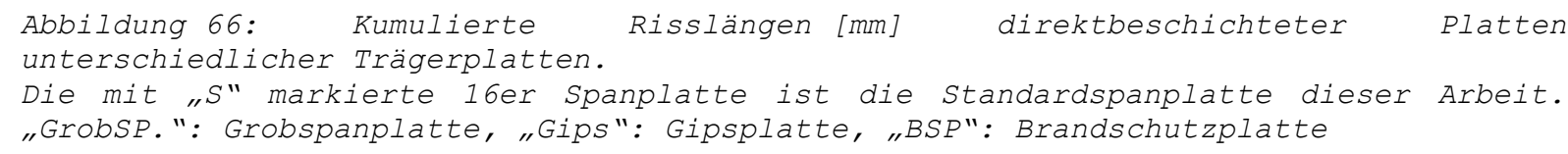

Die kumulierten Risslängen der beschichteten Spanplatten, MDF und Brandschutzplatte unterscheiden sich nicht signifikant (Abbildung 66). Die Grob-Spanplatte, Gipsplatte als auch beide Sperrhölzer zeigen eine erhöhte Rissanfälligkeit.

Bei der 9 mm-MDF spaltete sich ein Teil der Platte während des Heißverpressens. Proben wurden abseits dieser Stelle entnommen.

\section{DISKUSSION}

Die Platten wurden nach den empfohlenen Pressbedingungen des Imprägnierers beschichtet. Unter diesen Bedingungen führen Spanplatten, MDF und Brandschutzplatten als Trägerplatte nicht zu einer erhöhten Rissanfälligkeit der Direktbeschichtung. Bei Überhärtung, wenn die Direktbeschichtung rissanfälliger ist (Kapitel 5.1), könnte es eine Abhängigkeit dieser Trägerplatten zur Rissanfälligkeit geben.

Spalter bei der beidseitigen Beschichtung dünnen MDF sind ein von der Industrie bekanntes Problem. Es wird vermutet, dass bei der Verpressung entstehender Wasserdampf nicht zur Seite entweichen kann und das Material auseinander drückt.

Die hohe Rissanfälligkeit bei direktbeschichteten Sperrhölzern entspricht den Erwartungen der Industriepartner. Die unterschiedlichen Rissanfälligkeiten je nach Orientierung des Deckfurniers zur Produktionsrichtung des Imprägnats sind nicht gesichert. Die angegebenen Rissanfälligkeiten 
entsprechen dem arithmetischen Mittel von drei Proben derselben Platte. Auf Grund der Heterogenität von Sperrhölzern müssten für eine statistisch sichere Aussage deutlich mehr Proben geprüft werden. Der Zusammenhang erscheint dennoch logisch. Senkrecht zu den Holzfasern der Deckschicht entstehen die höchsten Quell- und Schwindbewegungen. Das Papier weist entlang der Produktionsrichtung die größten Festigkeiten auf. Liegen Papier und Deckschichtfasern parallel zueinander, liegen die hohen Quellbewegungen des Furniers entlang der geringeren Festigkeiten des Papiers. Viele Risse entstehen. Ausgehend von diesen Ergebnissen lässt sich eine Empfehlung für die Industrie ableisten: bei der Direktbeschichtung von Sperrhölzern sind senkrecht zueinander verlaufende Holz- und Papierfasern zu bevorzugen. Zwar treten beim verwendeten Material auch bei dieser Variante Risse auf, diese sind aber vermutlich dem Harz geschuldet. Die Industrie nutzt bei der Direktbeschichtung von Sperrhölzern darauf spezialisierte Harze. Das hier verwendete Harz gehört nicht zu diesen spezialisierten Harzen. Auch ein für Sperrhölzer spezialisiertes Harz könnte durch diese Orientierungspräferenz profitieren.

Die erhöhten Rissanfälligkeiten bei dieser Versuchsreihe sind vermutlich kleinflächigen Quell- und Schwindbewegungen geschuldet. Dadurch entstehen lokale hohe Spannung, die die Festigkeiten der Beschichtung übersteigen und zum Materialversagen führen. Bei den Sperrhölzern gibt der Jahrringverlauf im Deckfurnier eine Verteilung mechanischer als auch hygrischer Eigenschaften vor. Es kommt zu Flächen mit hohen und niedrigen Quellbewegungen. Bei der Grobspanplatte weisen die einzelnen Späne analog eines kleinen Furnierabschnitts variierende mechanische und hygrische Eigenschaften auf. Hinzu kommen abrupte Eigenschaftswechsel benachbarter Späne. Späne mit hohen Quellbewegungen können direkt neben Spänen mit niedrigen Quellbewegungen liegen. Bei Spanplatten und MDF könnten die Späne bzw. Faserbündel klein genug sein, um lokale Spannungsspitzen nicht auftreten zu lassen. Die Platten quellen und schwinden homogen über die gesamte Fläche. Gipsplatten sind von dieser Hypothese ausgeschlossen. Sie zeichnen sich durch ein verschwindend geringes Quellen und Schwinden aus. Daher lassen sich Quellbewegungen der Trägerplatte als Rissursache ausschließen. Wahrscheinlicher ist ein Versagen der Direktbeschichtung durch die eigenen Quell- und Schwindbewegungen auf dem starren Untergrund. 


\subsubsection{RISSPROJEKTION}

Um zu prüfen, ob Lage und Orientierung der Risse von der Trägerplatte vorgegeben werden, wurden Rissprojektionen durchgeführt. Dabei wird die Beschichtung einer Probe nach der Rissanfälligkeitsprüfung fotografiert, runtergeschliffen und erneut fotografiert. Mittels Bildbearbeitung werden die Risse der Direktbeschichtung auf die abgeschliffene Trägerplatte projiziert.

\section{ERGEBNISSE}

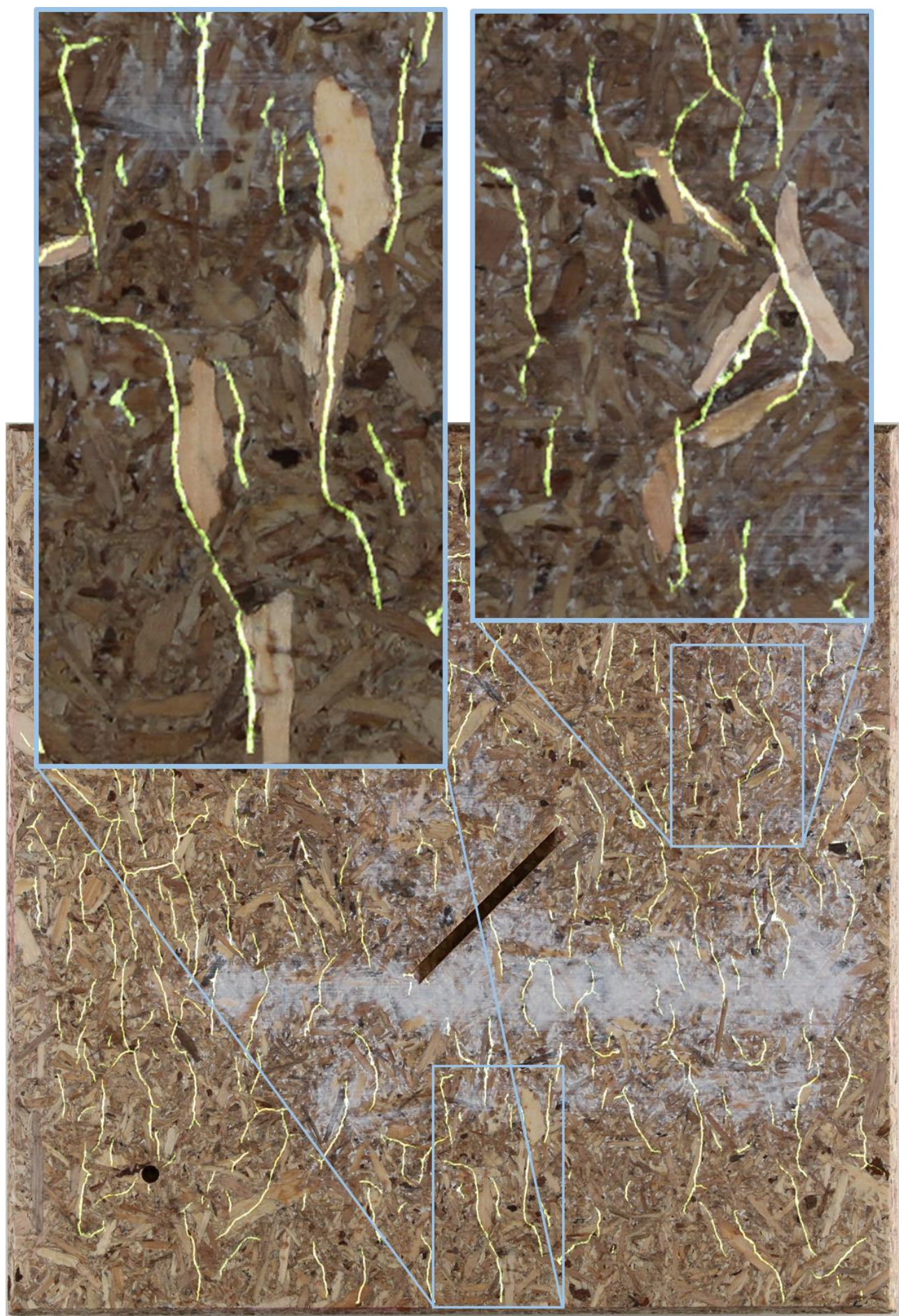

Abbildung 67: $25 \mathrm{~cm} \times 25 \mathrm{~cm}$ Rissanfälligkeitsprobe der Grobspanplatte nach der Prüfung.

In den beiden Nahaufnahmen sind einzelne Späne stärker kontrastiert dargestellt. 
Analog zu den Rissanfälligkeitsproben überhärteter Platten liegen auch bei diesen Proben die Risse vornehmlich abseits der Rissinitialen. Bei der Grobspanplatte liegen die Risse sowohl längs über Spänen als auch entlang von Spanränder. Es wurden keine Risse beobachtet, die quer über einen Span verlaufen (Abbildung 67). Bei beiden Sperrhölzern orientieren sich die Risse parallel zu den Fasern des Deckfurniers (Abbildung 68).
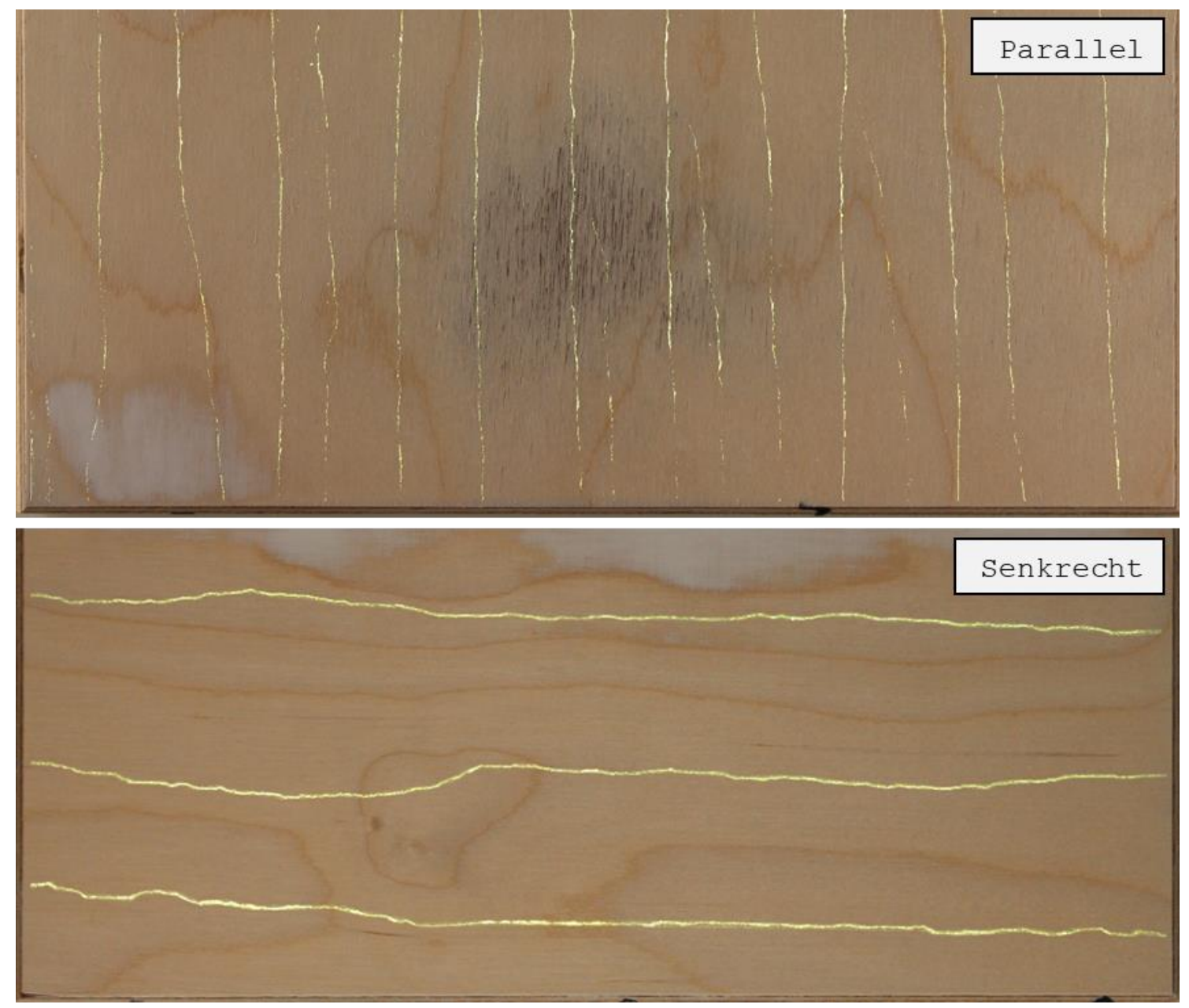

Abbildung 68: Aufnahmen der unteren Hälften von Rissanfälligkeitsproben der Sperrhölzer nach der Prüfung.

Bei beiden Aufnahmen verläuft die Produktionsrichtung des Papiers von oben nach unten. Im oberen Sperrholz ist die Faserorientierung des Deckfurniers parallel zum Papier, im unteren ist sie senkrecht zum Papier.

\section{DISKUSSION}

Holz weist quer zu den Fasern geringere Festigkeiten auf als längs der Fasern (NIEMZ 2012). Da sich Risse stets nach dem minimalsten Energieaufwand ausbreiten (STEIGEMANN, et al. 2010), reißt Holz längs der Fasern. Diese Neigung zeigt sich sowohl bei der Grobspanplatte als auch bei den Sperrhölzern. Quer zu den Holzfasern wurden keine Risse beobachtet.

Bei den restlichen Platten konnte keine von der Trägerplatte ausgehende Orientierung der Risse beobachtet werden. Dies kann durch deren Oberflächenstruktur erklärt werden. Die Späne bzw. Faserbündel sind zu klein, um den Rissen eine Richtung aufzuzwingen. Bei diesen Platten bestimmt die Produktionsrichtung des Papiers maßgeblich die Rissorientierung. 


\subsubsection{MIKRORISSE}

Bei einer Überhärtung kommt es zu vielen Mikrorissen in der Direktbeschichtung, die eine Materialschwächung bewirken. Es wurde geprüft, ob die Mikrorissdichte abhängig von der Trägerplatte ist. Dazu wurden angefärbte Mikrorisse unter einem Mikroskop vermessen und das Verhältnis aus kumulierter Risslänge zu untersuchter Fläche als Mikrorissdichte angegeben $(n=5)$.

\section{ERGEBNISSE}

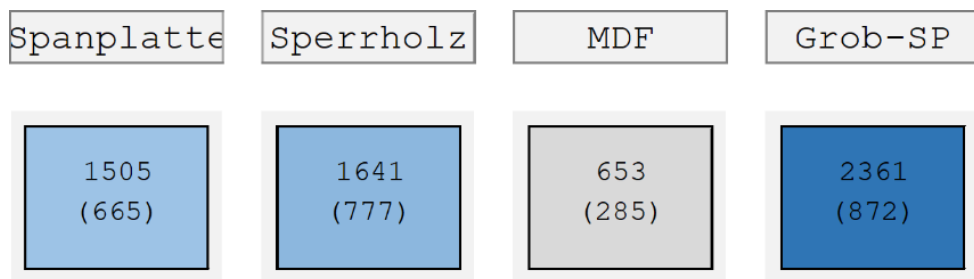

Abbildung 69: Mikrorissdichten [ $\left.\mathrm{\mu m} / \mathrm{cm}^{2}\right]$ direktbeschichteter Platten mit unterschiedlichen Trägerplatten.

In den Klammern stehen die standardabweichungen.

Die Mikrorissdichte bei MDF unterscheidet sich signifikant von denen anderer Trägerplatten. Es ist keine Orientierung der Mikrorisse an der Oberflächenstruktur der Trägerplatte zu erkennen (Abbildung 70 und Abbildung 71).

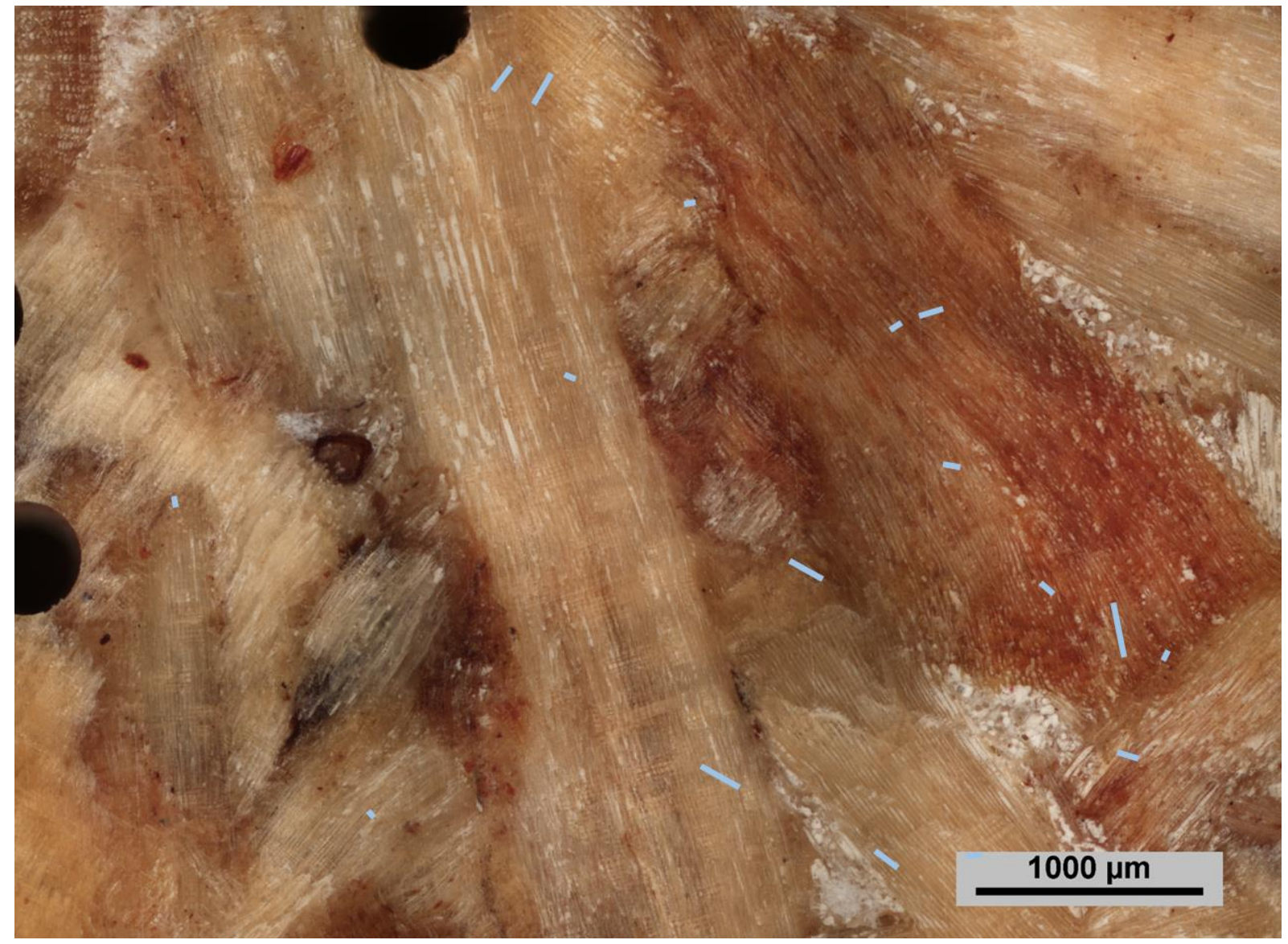

Abbildung 70: Mikrorissprojektion bei der Grobspanplatte. 


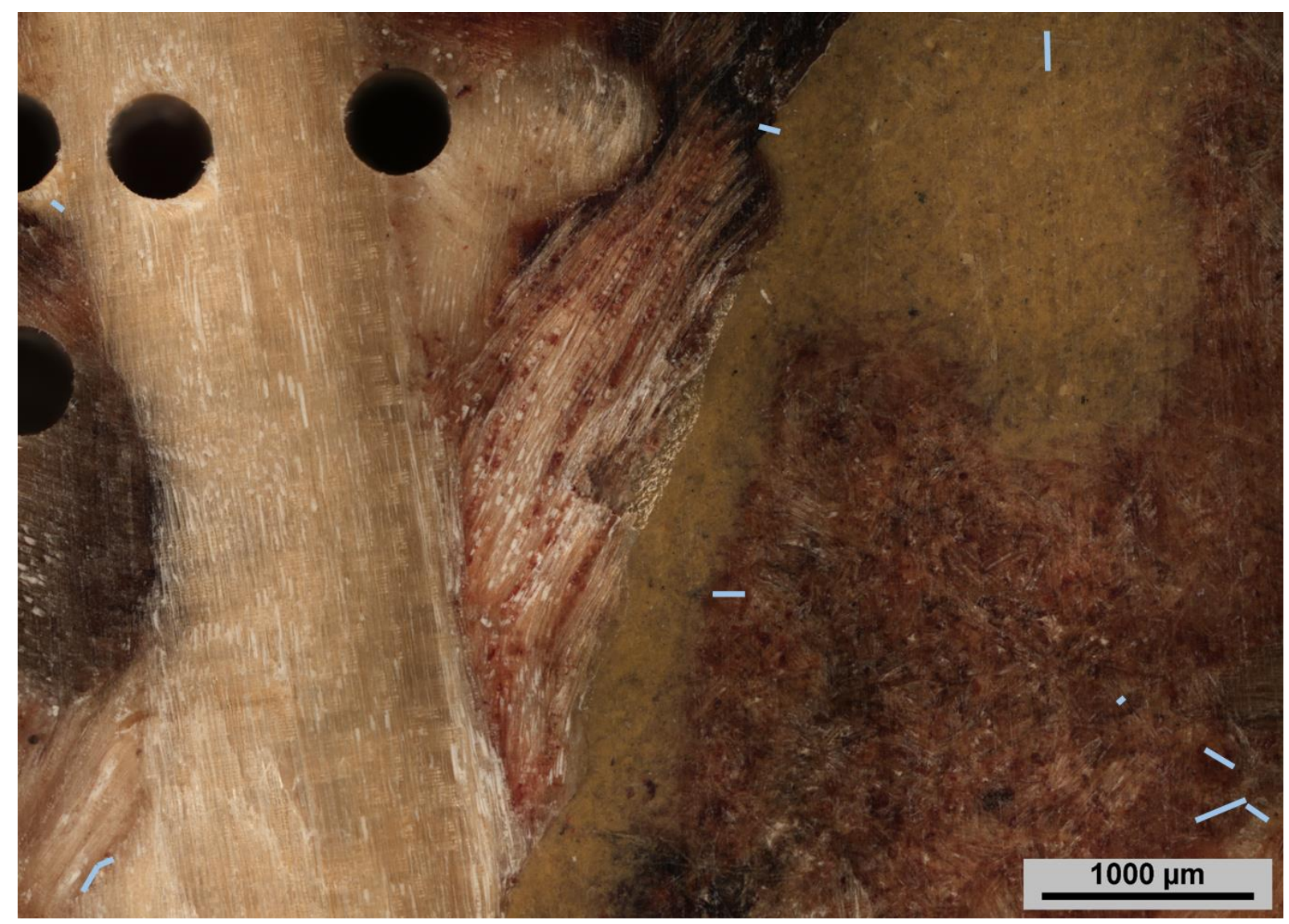

Abbildung 71: Mikrorissprojektion bei der Grobspanplatte.

\section{DISKUSSION}

Bei Spanplatten und Sperrhölzern sind die Mikrorissdichten gleich. Dennoch unterscheidet sich deren Rissanfälligkeit deutlich. Entsprechend kann die durch Mikrorisse hervorgerufene Festigkeitsabnahme (Kapitel 5.1.9) nicht maßgeblich für die erhöhte Rissanfälligkeit bestimmter Trägerplatten verantwortlich sein.

Die Lage und Orientierung von Makrorissen wird bei der Grobspanplatte und den Sperrhölzern von deren Oberflächenstruktur geprägt. Ein analoges Verhalten ist bei Mikrorissen nicht zu beobachten. Die lokalen Spannungsspitzen an Spanübergängen könnten sich über die Dicke der Direktbeschichtung verteilen. Direkt am Übergang von Trägerplatte zu Direktbeschichtung liegen die Spannungsspitzen vor. An der Oberfläche der Direktbeschichtung hingegen sind die Spannungen so weit verteilt, dass Mikrorisse sich nicht an der Trägerplattenstruktur orientieren. 


\subsubsection{MIKROSKOPIE}

Der Rissverlauf an den direktbeschichteten Trägerplatten wurde mittels Mikroskops untersucht.

\section{ERGEBNISSE}
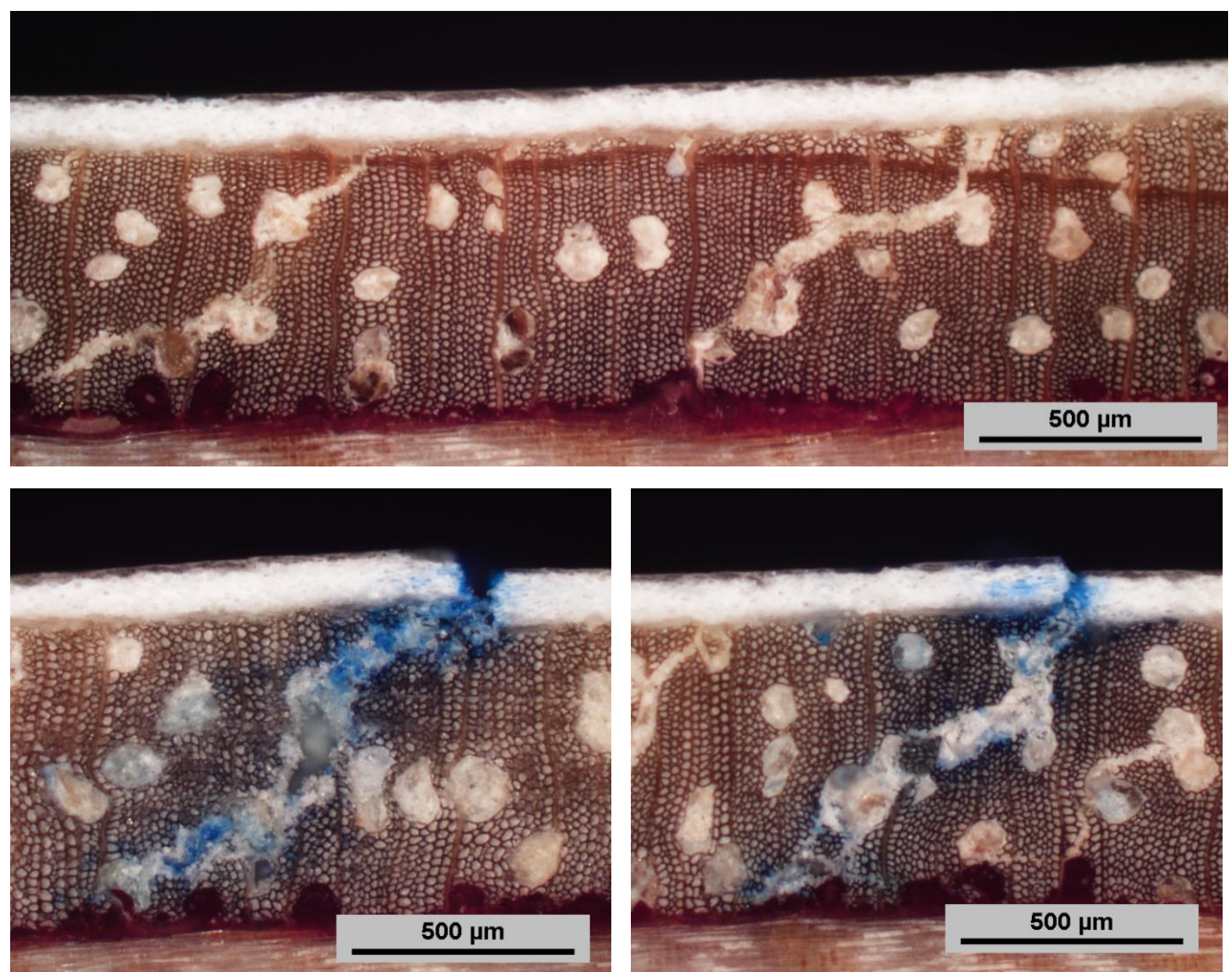

Abbildung 72: Querschnitte direktbeschichteter Sperrhölzer abseits von Rissen (oben) und an Rissen (unten).

Das Deckfurnier der Sperrholzplatte weist zahlreiche Schälrisse auf. Die Direktbeschichtung auf den Sperrhölzern reißt stets über einem solchen Furnierriss (Abbildung 72). Die Risse in der Grobspanplatte (Abbildung 73) drehen sich meist dicht unter der Grobspanplatte zur Seite und verlaufen fast waagerecht.

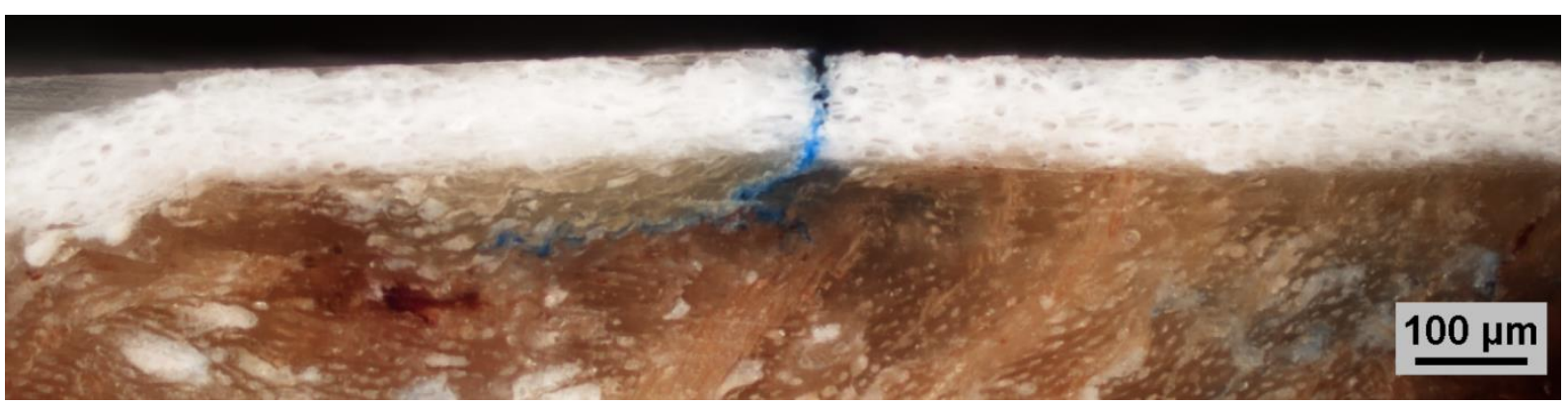

Abbildung 73: Riss in der Grobspanplatte.

\section{DISKUSSION}

Bei der industriellen Produktion von Schälfurnieren kommen stets Schälrisse vor. Es gibt unterschiedliche Ansichten, wie mit Schälrissen umgegangen werden sollte. So gibt es die Meinung, 
Furniere sollen so gedreht werden, dass die einseitig auftretenden Schälrisse auf der abgedeckten Rückseite liegen. Andere Meinungen vertreten eine gegensätzliche Drehung. Begründet wird dies mit dem Entfernen bzw. Freilegen der Schälrisse beim Abschleifen. Liegen die Risse auf der Rückseite des Furniers, führt ein Abschleifen zum Freilegen der Risse. Liegen sie auf der Oberseite werden sie weggeschliffen. (LUKOWSKY 2015)

Die Risse der Direktbeschichtung liegen beim Sperrholz stets über Schälrissen. Ein Einfluss der Schälrisse auf die Rissanfälligkeit ist wahrscheinlich. An Schälrissen weist das Furnier zum einen eine Sollbruchstelle auf. So sinkt bspw. die Biegefestigkeit durch Schälrisse (RAHAYU, et al. 2015). Zum anderen kann die Direktbeschichtung Kräfte nicht mehr in den Untergrund ableiten. Durch das Beschichten eines schälrissenfreien Holzes könnte man diese Theorie überprüfen. Dafür würde sich bspw. ein dünngeschliffenes Vollholzbrett oder Sägefurnier eignen.

\subsubsection{EIGENSPANNUNGEN}

Spannungen im System können zum Materialversagen führen. Es sollte geprüft werden, ob bei Trägerplatten mit einer hohen Rissanfälligkeit eine hohe Eigenspannung vorliegt. Dazu wurden Proben $(n=5)$ bestehend aus Spanplattendeckschicht und Direktbeschichtung erst bei 30\% rel. Lf., dann $85 \%$ rel. Lf. klimatisiert und das Stichmaß der verformten Proben als Maß für deren Eigenspannung vermessen.

\section{ERGEBNISSE}

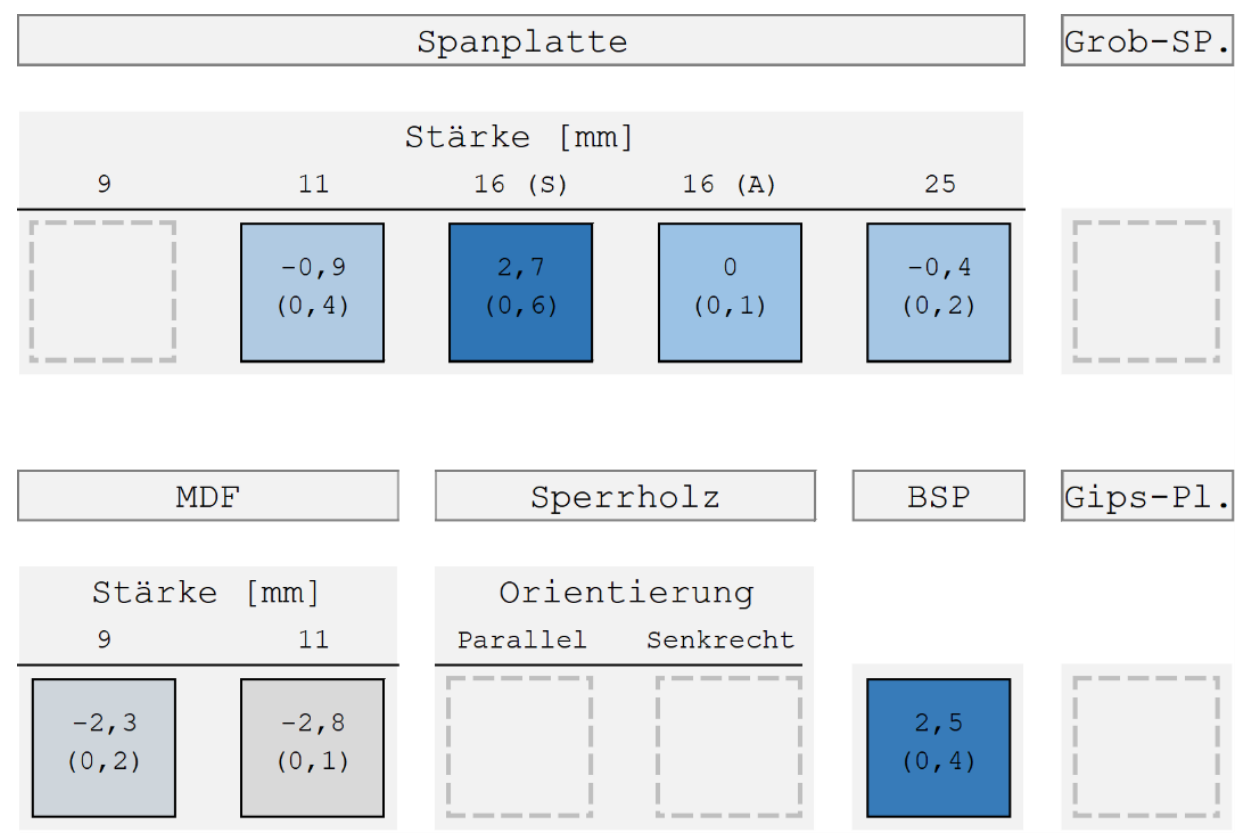

Abbildung 74: Änderungen des Stichmaßes [mm], wenn die relative Luftfeuchte von 30\% auf $85 \%$ erhöht wird.

In den Klammern stehen die Standardabweichungen.

Bei der Grobspanplatte, den Sperrhölzern und der Gipsplatte entstanden bereits vor der Prüfung Risse in der Direktbeschichtung. Da dies einen Spannungsabbau und damit eine Stichmaßänderung nach sich zieht, wurden die Stichmaße dieser Platten nicht gemessen.

Dargestellt sind Stichmaßänderungen bei einer Erhöhung der relativen Luftfeuchte von 30\% auf 85\% (Abbildung 74). Negative Werte entsprechen einer Verformungsabnahme. In dem Fall quillt die Trägerplatte beim Klimawechsel geringer als die Beschichtung. Lediglich die Standard-Spanplatte, die bei den restlichen Versuchsreihen verwendet wurde und die Brandschutzplatte zeigen positive Werte. 
Bei keiner Probe wurde ein Wechsel der Formungsrichtung von konkav zu konvex oder umgekehrt beobachtet.

\section{DISKUSSION}

Die alternative $16 \mathrm{~mm}$-Spanplatte gilt auf Grund der feinen Deckschicht und des geringen Recyclinganteils als eine Premiumspanplatte für die Direktbeschichtung. Als einzige zeigt sie keinerlei Änderung der Krümmung. Bei Erhöhung der relativen Luftfeuchte von 30\% auf $85 \%$ quellen Spanplatte und Direktbeschichtung gleich. Die beiden MDF quellen geringer als die Direktbeschichtung, das Stichmaß geht zurück.

Bei Platten mit hoher Rissanfälligkeit konnten keine verwertbaren Stichmaße gemessen werden. Daher kann kein Vergleich zwischen rissempfindlichen und rissunempfindlichen Platten hinsichtlich der Eigenspannung gezogen werden. Es ist möglich, dass rissanfälligen Platten eine hohe Eigenspannung aufwiesen und dadurch bereits vor der Prüfung gerissen sind. Aus diesem Grund ist die Eigenspannung als Einflussgröße auf die Rissanfälligkeit nicht auszuschließen.

\subsubsection{ABSCHLIEßENDE DISKUSSION ZUR VERSUCHSREIHE}

Bei überhärteten Direktbeschichtung oder ungünstigen Feuchtekonstellationen von Spanplatte und Imprägnat schwächen Mikrorisse die Direktbeschichtung. Bei 2-blättrigen Direktbeschichtungen kommt es durch höhere hygrische Längenänderungen der Direktbeschichtung zu Spannungen. Beide Hypothesen erklären nicht die Rissanfälligkeit bei dieser Versuchsreihe; die Imprägnate wurden gleich ausgehärtet, Mikrorisse liegen nicht in einem Umfang vor, der die Rissanfälligkeit erklärt und die Materialien waren gleich klimatisiert.

Bei der Oberfläche von Sperrhölzern und der Grobspanplatte kommt es zu kleinflächigen Quell- und Schwindbewegungen, die die Direktbeschichtung unter Spannungen setzen. Durch diese Spannungsspitzen kommt es bei der Rissanfälligkeitsprüfung zu einem Reißen der Direktbeschichtung. Die Orientierung der Makrorisse bekräftigt diese Hypothese. Bei Sperrhölzern schwächen die Schälrisse zusätzlich das Deckfurnier.

Quellbewegungen könnten mittels Digital Image Correlation (kurz DIC) aufgenommen werden. Bei dieser Methode werden vor und nach einer Belastung hochauflösende Bilder aufgenommen. Über eine vergleichende Auswertung lassen sich selbst kleinflächige Verformungen (Quell- und Schwindbewegungen) darstellen. 


\section{ZUSAMMENFASSUNG}

In der vorliegenden Arbeit wurden erstmalig die Einflussgrößen auf die Rissanfälligkeit von Melaminharz-Direktbeschichtungen systematisch untersucht. Dazu wurden Methoden zur Quantifizierung der Rissanfälligkeit, zur Quantifizierung des Aushärtungsgrads und zur Bestimmung der mechanischen Eigenschaften entwickelt.

Die bestehende Normprüfung zur Evaluierung der Rissanfälligkeit (DIN EN 14323:2014) erlaubt keine für die bearbeitete Fragestellung ausreichenden Differenzierung. Durch Einbringen von Rissinitialen in die Prüfkörper und Änderung der Bewertungsgrundlage konnte die Differenzierbarkeit deutlich erhöht werden. Bei den Rissinitialen handelt es sich um praxisübliche Bearbeitungen (Nut für Flachpressdübel und Bohrung für Bodenträger) einer direktbeschichteten Platte. Entsprechend zeigt dieses Verfahren eine praxisrelevante Rissanfälligkeit der Platten auf. Als Bewertungsgrundlage wurde die Klassifizierung des Rissbilds durch eine Vermessung sämtlicher Risslängen ersetzt. Als Indikator für die Rissanfälligkeit dient die kumulierte Risslänge, die eine gute Differenzierung zulässt und bei der es im Gegensatz zu anderen untersuchten Indikatoren nicht zu einer Unterschätzung der Rissanfälligkeit bestimmter Rissbilder kommt. Mit der so modifizierten Prüfung lassen sich selbst Platten, denen die Norm denselben Risswiderstand attestiert, differenzieren. Weitere Untersuchungen zur Bestimmung der Rissanfälligkeit thematisierten den Einfluss des Raumklimas, der Belastungsdauer und -höhe sowie der Materialnutzung. Das Raumklima hat bei den gewählten Rahmenparametern nur einen sehr geringen Einfluss auf die Prüfergebnisse. Änderungen der Normvorgaben zur Belastungsdauer und -temperatur beeinflussen zwar die gemessene Rissanfälligkeit, erlauben jedoch keine bessere Differenzierung. Es ist möglich, beide Seiten einer Platte an derselben Probe zu prüfen und dadurch das Untersuchungsmaterial effizienter zu verwerten. Allerdings steigt dadurch die gemessene Rissanfälligkeit im Vergleich zur Prüfung beider Seiten an separaten Prüfkörpern.

Vom Aushärtungsgrad einer Direktbeschichtung sind viele Eigenschaften abhängig: die Adhäsion zur Trägerplatte, Härte, Glanz, Medienresistenz, Kantenausbrüche beim Zuschnitt und offene Poren an der Oberfläche. In der Industrie haben sich firmeneigene Abwandlungen des Kiton-Tests (AS/NZS 4266.21:2004) zur Messung des Aushärtungsgrades etabliert. Diese Methoden sind jedoch nur subjektiv auswertbar, sie erlauben keine gute Differenzierung und die Prüfergebnisse sind beeinflusst durch die Harzüberdeckung der Papierfasern und der Raumtemperatur. In dieser Arbeit wurde eine Methode entwickelt, mit der eine bessere Differenzierung möglich ist. Bei der so genannten Pulverhydrolyse wird ein Pulver der Direktbeschichtung mit Salzsäure hydrolysiert und die Lösung UV-spektroskopisch vermessen. Untersuchungen zu der Methode zeigen, dass das UV-Spektrum der Pulverhydrolyse durch methyloliertes Melamin hervorgerufen wird. Das Spektrum der Hydrolyselösungen verändert sich nicht innerhalb der beobachteten 4 Tage bei der für diese Prüfung relevanten Wellenlänge von $237 \mathrm{~nm}$. Ein strikt einzuhaltender zeitlicher Ablauf der Methode ist daher nicht notwendig. Umgebungstemperatur, Säurekonzentration und Hydrolysedauer haben einen Einfluss auf das Ergebnis der Methode. Wird die Hydrolyse bei $40^{\circ} \mathrm{C}$ durchgeführt, lässt sich die Methode von 2 Stunden auf 5 Minuten verkürzen und Schwankungen der Raumtemperatur fallen als Störgrößen weg. Eine Reinigung der Direktbeschichtung mit Ethanol, Aceton, Wasser oder Papiertüchern vor Durchführung der Hydrolyseprüfung hat keinen Einfluss auf das Messergebnis. Mit der entwickelten Puvlerhydrolyse lässt sich der Aushärtungsgrad gut differenziert, objektiv und deutlich schneller bestimmen als mit den bisherigen Prüfmethoden.

Um die mechanischen Eigenschaften einer Beschichtung abzuschätzen, kann eine Biegeprüfung an damit beschichteten Trägerplatten durchgeführt werden. Auf Grund mechanischer und geometrischer Zusammenhänge ist der Einfluss der Beschichtung auf die Biegeeigenschaften deutlich messbar. 
Allerdings werden diese Biegeeigenschaften auch durch die mechanischen Eigenschaften der beschichteten Trägerplatte beeinflusst. Erstmals wurde ein Model angewendet, mit dem der Einfluss der Trägerplatte weitgehend eliminiert und die mechanischen Kennwerte der Beschichtung bestimmt werden können. Das Model nutzt mehrere geometrische und mechanische Kenndaten, die zum Großteil bei der Biegeprüfung aufgenommen werden. Lediglich die Schichtdicken müssen separat aufgenommen werden. Realistische Schwankungen der Eingabewerte auf Grund von Messfehlern haben nur einen geringen Einfluss auf das Model.

Allgemein lässt sich eine Rissentstehung auf zu hohe Spannungen bzw. zu geringe Festigkeiten zurückführen. Für verschiedene Einflussgrößen wurde untersucht, inwiefern sich Materialeigenschaften verändern und dadurch eine entsprechende Spannungszunahme bzw. Festigkeitsabnahme auslösen können.

Bei einer Überhärtung steigt die Rissanfälligkeit einer Direktbeschichtung an. Dies lässt sich auf Mikrorisse zurückführen. Die Mikrorissdichte einer Platte steigt mit zunehmendem Aushärtungsgrad an. Sowohl Biegeprüfungen an direktbeschichteten Platten als auch das Model zur Berechnung der mechanischen Eigenschaften der Direktbeschichtung zeigen eine durch Mikrorisse ausgelöste Festigkeitsabnahme. Durch die Festigkeitsabnahme kommt es bei überhärteten Direktbeschichtungen vermehrt zur Rissbildung.

2-blättrige Direktbeschichtungen haben eine höhere Rissanfälligkeit als 1-blättrige. Zwei voneinander unabhängige Methoden zeigen ein ausgeprägteres hygrisches Verhalten bei 2-blättrigen Direktbeschichtung. Wodurch dies hervorgerufen wird, konnte nicht herausgefunden wurden. Dieses Verhalten könnte die Rissanfälligkeit hervorrufen. Die durch hohe hygrische Längenänderungen induzierten Spannungen könnten die Festigkeit der 2-blättrigen Direktbeschichtungen überschreiten und somit Risse verursachen.

Werden Imprägnate und Spanplatten unkontrolliert gelagert, können sich deren Materialfeuchten unterscheiden. Untersuchungen bestätigen einen Einfluss sowohl der Imprägnatsfeuchte als auch der Spanplattenfeuchte auf die Rissanfälligkeit der Direktbeschichtung. Je feuchter das Imprägnat und je trockener die Spanplatte, desto höher ist die Rissanfälligkeit. Diese Rissanfälligkeit lässt sich auf Quellund Schwindbewegungen zurückführen. Bei der rissanfälligsten Platte ist ein gequollenes Imprägnat auf einer geschwundenen Spanplatte verpresst. Akklimatisieren sich die Materialien, schwindet das Imprägnat und die Spanplatte quillt. Diese gegenläufige Dimensionsänderungen induzieren Spannungen zwischen den Schichten und es treten Mikrorisse auf, welche die Festigkeit der Direktbeschichtung verringern.

Aus Schadensanalysen ist bekannt, dass Löcher in der Spanplatte zu Rissen in der darüber liegenden Direktbeschichtung führen können. Um diesen Einfluss zu untersuchen, wurden vor dem Verpressen unterschiedliche Defekte in die Spanplattenoberfläche eingebracht. Allerdings wurde bei dem Untersuchungsmaterial unbeabsichtigt ein Rissanfälligkeitsgradient in die Platte eingebracht. Proben aus der Plattenmitte sind rissanfälliger als Proben vom Plattenrand. Dadurch lässt sich der Einfluss der verschiedenen Defekttypen nicht vergleichen. An diesem Material entstandene Risse liegen stets abseits der Defekte. Selbst in der rissanfälligen Plattenmitte kommt es nicht zur erwarteten Rissbildung über den Defekten. Der Widerspruch zwischen Beobachtungen aus Schadensfällen und dem Verhalten der untersuchten Platten ließ sich im Rahmen der Arbeit nicht klären.

Direktbeschichtungen auf Grobspanplatten und Sperrhölzern haben höhere Rissanfälligkeiten als Direktbeschichtungen auf Spanplatten oder MDF. Zwar wurden Mikrorisse dokumentiert, deren Dichte von der Trägerplatte abhängig ist. Allerdings kommen bei keiner Platte Mikrorissdichten in einer Höhe 
vor, die die Rissanfälligkeit erklären könnte. Bei der Grobspanplatte liegen die Risse entlang oder zwischen Spänen. Jeder Span quillt und schwindet. Zwischen benachbarten Spänen können abrupte Eigenschaftswechsel vorkommen. An diesen Stellen könnten Spannungsspitzen die Festigkeit der Direktbeschichtung übersteigen. Bei Sperrhölzern orientieren sich die Risse an der Faserorientierung des Deckfurniers. Quer zu den Fasern weist Holz sowohl die höchsten Quell- und Schwindbewegungen als auch die niedrigste Festigkeit auf. Zusätzlich ist das Deckfurnier von Schälrissen überzogen. Diese fungieren als Sollbruchstellen. Risse der Direktbeschichtung setzen sich stets in einen solchen Schälriss fort.

Um eine Direktbeschichtung mit einer geringen Rissanfälligkeit zu produzieren gibt es mehrere Vorrausetzungen:

1. Die Direktbeschichtung sollte nicht überhärtet sein.

2. Die Direktbeschichtung sollte das gleiche hygrische Verhalten aufweisen wie die Trägerplatte.

3. Die Materialfeuchten des Imprägnats und der Trägerplatte sollten zum Zeitpunkt der Verpressung aufeinander abgestimmt sein.

4. Löcher, Risse oder andere Defekte in der Trägerplatte sollten nicht vorkommen.

5. Die Trägerplatte sollte eine homogene, feine Oberflächenstruktur aufweisen.

Wird bereits einer dieser Punkte nicht eingehalten, kann dies zu einer erhöhten Rissanfälligkeit der Direktbeschichtung kommen. Wichtig für eine geringe Rissanfälligkeit ist ein gutes Zusammenspiel aus Direktbeschichtung und Trägerplatte. 


\section{QUeLLENVERZEICHNIS}

\subsection{LITERATUR}

Anonymus (Unbekannt)

Prüfvorschrift WKI - Bestimmung des Aushärtungsgrades an mit Melaminharz vergüteten

Holzwerkstoffen.

Anonymus (2012)

HPL/CPL: Marktwachstum verlagert sich nach Osten

Ergebnisse einer neuen Studie wurden auf der ICDLI-jahrestagung vorgestellt

Euwid 48.

BATCHELOR, et al. (2006)

Effect of test conditions on measured loads and displacements in zero-span testing

Tappi journal Vol. 5, No. 10.

BÖTTCHER; SAGROSKE (1985)

Rissbildung bei melaminharzbeschichteten Holzspanplatten infolge Über- oder Unterhärtung des Harzes 30/85.

CARDARELLI (2018)

Materials Handbook

Springer International Publishing(Cham); ISBN: 978-3-319-38923-3.

CASEY (1980)

Pulp and paper

Wiley(New York, Chichester); Seiten: XXII, S. 821-1446; ISBN: 978-0-471-03176-5.

CZICHOS; HENNECKE (2004)

HÜTTE - Das Ingenieurwissen

Springer-Verlag Berlin Heidelberg(Berlin, Heidelberg); ISBN: 978-3540203254.

Diem; MatTHIAS; WAGNeR (2000)

Amino Resins.

EBDON; HUNT; O'ROURKE (1987)

Characterisation of separated melamine-formaldehyde adducts (methylolmelamines) and adduct mixtures by h.p.l.c. and by n.m.r. and u.v. spectroscopy

British Polymer Journal 2; Band: 19. 197-203.

EGGER (2019)

Technisches Datenblatt - Eurospan E1E05 TSCA P2 CE EAC (Rez. 181).

EGGER (2020a)

Technisches Datenblatt - Eurospan E1E05 Hydro P3 CE EAC.

EGGER (2020b)

Technisches Datenblatt - Eurospan FLAMMEX E1E05 P2 CE EAC.

EYERER; ELSNER; HIRTH (2005)

Die Kunststoffe und ihre Eigenschaften

Springer-Verlag(s.l.); Seiten: 1633; ISBN: 3-540-21410-0. 
Freedonia Group (2014)

World Decorative Laminates

Industry Study with Forecasts for 2018 \& 2023.

GAUSSORGUES (1994)

Infrared Thermography

Springer Netherlands(Dordrecht); Seiten: 1 Online-Ressource (XV, 508 p); ISBN: 9789401107112.

GoloMBEK (Unbekannt)

PAPER TESTING DEVICE

Device and method for measuring the curing and the condensation properties of reaction resins (e.g. aminoplast, epoxy, polyester, polyurethane etc.) of impregnated papers, fleeces, tissues etc.

GRIFFITH (1921)

The Phenomena of Rupture and Flow in Solids

Philosophical Transactions of the Royal Society of London 221. 163-198.

GRUBBS (1969)

Procedures for Detecting Outlying Observations in Samples

Technometrics Vol. 11, No. 1.

GUENTHER (1988)

Schnelle Methode zum Bestimmen des Aushärtegrads von MF- und MPF-Formmassen.

Kunststoffe 4; Band: 78. 328-329.

GURNAGUL; PAGE (1989)

The difference between dry and rewetted zero-span tensile strength of paper

Tappi journal Vol. 72.

HAGSTRAND; OKSMAN (2001)

Mechanical Properties and Morphology of Flax Fiber Reinforced Melamine-Formaldehyde

Composites

Polymer Composites Vol. 22 No. 4. 568-578.

HIMMEL; MAI (2015)

Effects of acetylation and formalization on the dynamic water vapor sorption behavior of wood Holzforschung 5; Band: 69. 99.

HOLIK (2013)

Taschenbuch der Papiertechnik, Kapitel 10

CARL HANSER Verlag GMBH \&([Place of publication not identified]); ISBN: 978-3-446-43802-6.

ICDLI (2017)

ICDLI Market Research.

KANDELBAUER, et al. (2009a)

Model-free kinetic analysis of melamine-formaldehyde resin cure

Chemical Engineering Journal 2-3; Band: 152. 556-565.

KANDELBAUER, et al. (2009b)

On the performance of a melamine-urea-formaldehyde resin for decorative paper coatings

European Journal of Wood and Wood Products 1; Band: 68. 63-75. 
KANDELBAUER, et al. (2010)

Möglichkeiten der Online-NIR-Spektroskopie im Bereich der Fertigungsindustrie am Beispiel der umfassenden Produktionskontrolle von dekorativen Schichtstoffen

Chemie Ingenieur Technik 4; Band: 82. 537-544.

KIM; NAIRN (2000)

Fracture mechanics analysis of coating/substrate systems

Part II: Experiments in bending

Engineering Fracture Mechanics. 1-25.

KLEPPMANN (2016)

Versuchsplanung

Produkte und Prozesse optimieren

Hanser(München, Wien); Seiten: 349; ISBN: 978-3-446-44716-5.

KOHLMAYR, et al. (2013)

Drying and curing behaviour of melamine formaldehyde resin impregnated papers

Journal of Applied Polymer Science 3; Band: 131. n/a-n/a.

KRASNOSHLYK (2018)

Etude multi-échelles et multiphysiques des méchanismes de fissuration dans les matériaux à base de fibres naturelles().

LAZARUS; PAUCHARD (2011)

From craquelures to spiral crack patterns

Influence of layer thickness on the crack patterns induced by desiccation

Soft Matter 6; Band: 7. 2552.

LUKOWSKY (2015)

Failure analysis of wood and wood-based products

McGraw-Hill Education(New York, Chicago, San Francisco); Seiten: 220; ISBN: 9780071839372.

LUKOWSKY (2016)

Produktanalyse und Entwicklung eines Verfahrens zur Prüfung der Verklebung von

Mehrschichtparketten unter besonderer Berücksichtigung des Verhaltens bei Renovierungen mit wasserbasierten Beschichtungen

IGF-Vorhaben Nr. 17856 N.

MAI; MILITZ (2012)

Taschenbuch der Holztechnik, Kapitel 4.3

Fachbuchverl. Leipzig (München); Seiten: 568; ISBN: 978-3-446-42605-4.

MANG; HOFSTETTER (2018)

Festigkeitslehre

Springer Vieweg (Berlin); Seiten: XV, 584 strony; ISBN: 3662575647.

MATTHECK; Bethge (2003)

Warum alles kaputt geht

Form und Versagen in Natur und Technik

Forschungszentrum Karlsruhe(Karlsruhe); Seiten: 208; ISBN: 3-923704-41-0.

MERLINE; VUKUSIC; ABDAla (2012)

Melamine formaldehyde 
Curing studies and reaction mechanism

Polymer Journal 4; Band: 45. 413-419.

MORATH; WOODS (1958)

Analysis of Amino-Formaldehyde Resins

Analytical Chemistry 8. 1437-1440.

NEMLI; ÇOLAKOG ${ }^{2} \mathrm{LU}$ (2005)

The influence of lamination technique on the properties of particleboard

Building and Environment 1; Band: 40. 83-87.

NIEMz (2012)

Taschenbuch der Holztechnik, Kapitel 1.4

Fachbuchverl. Leipzig (München); Seiten: 568; ISBN: 978-3-446-42605-4.

NOWAK-OSSORIO; BRAUN (1982)

Bestimmung stickstoffhaltiger Bindemittelkomponenten in Holzspanplatten

Holz als Roh- und Werkstoff 40. 255-259.

OEHLER (2016)

Schwindung und Verzug von harzbasierten Laminaten. Abschlussbericht Aif $1757 \mathrm{~N}($ ).

OHLMEYER (2002)

Untersuchung über die Eigenschaftsentwicklung von Holzwerkstoffplatten nach dem Heißpressen().

PAGE (1969)

A Theory for the tensile strength of paper

Tappi journal Vol. 52 No. 4. 674-681.

PFLEIDERER (2020a)

Technisches Datenblatt - ClassicBoard p3.

PFLEIDERER (2020b)

Technisches Datenblatt - ClassicBoard P2.

PULMAC INTERNATIONAL (Unbekannt)

Z-Span 1000 Operation and Service Manual.

RAHAYU, et al. (2015)

The Effect of Jabon Veneer Quality on Laminated Veneer Lumber Glue Bond and Bending Strength

J. Ilmu Teknol Vol. 13 No. 2.

RAUCH HOLZWERKSTOFFE (Unbekannt)

Daten und Fakten

Prospekt zu WOODMAX M und WOODMAXX Spanplatten.

ROFFAEL (2004)

Untersuchungen zur Verminderung der Längenänderung von Holzspanplatten durch gezielte Nutzung von materialimmanenten Eigenschaften und Verwendung von feuchtebeständigen Zusatzstoffen Schlussbericht(Projektnummer 0339851/8). 
SCHINDLBAUER; ANDERER (1980)

Eine einfache Charakterisierung von Melamin-Formaldehyd-Kondensaten mit Hilfe von NMR-

Messungen

Fresenius Zeitung für Analytische Chemie 301. 210-214.

SCHRÖDER; FRANZ; HAGEN (1976)

Ausgewählte Methoden zur Plastanalytik

Akademie-Verlag(Berlin).

SCHWAB; STEFFEN; KORTE (1997)

Feuchtebedingte Längenänderungen von Holzwerkstoffen in Plattenebene

European Journal of Wood and Wood Products 2; Band: 55. 227-233.

SONDEREGGER; NieMz (2006)

Untersuchungen zur Quellung und Wärmedehnung von Faser-, Span- und Sperrholzplatten

European Journal of Wood and Wood Products 1; Band: 64. 11-20.

SONDEREGGER; NIEMZ (2009)

Thermal conductivity and water vapour transmission properties of wood-based materials

European Journal of Wood and Wood Products 3; Band: 67. 313-321.

STEIGEMANN, et al. (2010)

Simulation of crack paths in functionally graded materials

Engineering Fracture Mechanics 11; Band: 77. 2145-2157.

TAPPI 494 ()

Tensile properties of paper and paperboard.

THOLE (1991)

Dimensionsänderungen von Möbelspanplatten verschiedener Hersteller in drei

Raumrichtungen 28/91.

WASHINGTON, et al. (2009)

The use of near-IR and differential scanning calorimetry to develop models that predict the extent of phenolic resin curing in saturating kraft paper

Papel 10; Band: 70. 62-71.

ZEPPENFELD; GRUNWALD (2005)

Klebstoffe in der Holz- und Möbelindustrie

DRW-Verlag Weinbrenner(Leinfelden-Echterdingen); Seiten: 352; ISBN: 3871813591.

\subsection{NORMEN UND PATENTE}

AS/NZS 4266.0 (2004)

Reconstituted wood-based panels - Methods of test.

AS/NZS 4266.21 (2004)

Reconstituted wood-based panels-Methods of test - Method 21: Over-cure or under-cure of resin.

DIN 53107 (2016)

Prüfung von Papier und Pappe - Bestimmung der Glätte nach Bekk. 
DIN EN 310 (1993)

Holzwerkstoffe - Bestimmung des Biege-Elastizitätsmoduls und der Biegefestigkeit.

DIN EN 318 (2002)

Holzwerkstoffe - Bestimmung von Maßänderungen in Verbindung mit Änderungen der relativen Luftfeuchte.

DIN EN 13183-1-Ber (2003)

Feuchtegehalte eines Stückes Schnittholz.

DIN EN 13329 (2017)

Laminatböden - Elemente mit einer Deckschicht auf Basis aminoplastischer, wärmehärtbarer Harze Spezifikationen, Anforderungen und Prüfverfahren.

DIN EN 14323 (2014)

Holzwerkstoffe - Melaminbeschichtete Platten zur Verwendung im Innenbereich - Prüfverfahren.

DIN EN ISO 527-3 (2003)

Kunststoffe Bestimmung der Zugeigenschaften Teil 3: Prüfbedingungen für Folien und Tafeln.

DIN EN ISO 536 (2012)

Papier und Pappe - Bestimmung der flächenbezogenen Masse.

DIN EN ISO 12572 (2017)

Wärme- und feuchtetechnisches Verhalten von Baustoffen und Bauprodukten.

DIN ISO 3781 (2012)

Papier und Pappe - Bestimmung der breitenbezogenen Bruchkraft nach dem Eintauchen in Wasser.

ISO 2144 (2015)

Paper, board, pulps and cellulose nanomaterials - Determination of residue (ash content) on ignition at $900^{\circ} \mathrm{C}$.

ISO 5636-5 (2013)

Paper and board - Determination of air permeance (medium range) - Part 5: Gurley method.

ISO 6588-2 (2012)

Paper, board and pulps - Determination of $\mathrm{pH}$ of aqueous extracts - Part 2: Hot extraction.

EP 3238934 A1 (2017). BRAUn; DENK; KaLWA

Verfahren zur Bestimmung des Aushärtungsgrades von mindestens einer auf einer Trägerplatte angeordneten Polymerschicht.

US 5001068 (1991). GOLOMBEK

METHOD FOR DETERMINING SOLIDIFICATION DEGREE OF CARRIER IMPREGNATED WITH REACTION RESIN. 
ANHANG

ANOVA-MODEL: HYDROLYSIERTES MELAMIN IN ABHÄNGIGKEIT VON HYDROLYSEDAUER, SÄUREKONZENTRATION UND UMGEBUNGSTEMPERATUR

\begin{tabular}{|c|c|c|c|c|c|}
\hline \multirow[b]{2}{*}{ Source } & \multicolumn{2}{|l|}{ Sum of } & \multirow{2}{*}{$\begin{array}{r}\text { Mean } \\
\text { Square }\end{array}$} & \multirow{2}{*}{$\begin{array}{r}F \\
\text { Value }\end{array}$} & p-value \\
\hline & Squares & $d f$ & & & Prob > F \\
\hline Model & 1706,07 & 7 & 243,72 & 401,11 & $<0.0001$ \\
\hline A-Dauer & 632,50 & 1 & 632,50 & 1040,93 & $<0.0001$ \\
\hline B-Konzentration & 136,62 & 1 & 136,62 & 224,84 & $<0.0001$ \\
\hline C-Temperatur & 409,26 & 1 & 409,26 & 673,53 & $<0.0001$ \\
\hline$A B$ & 4,53 & 1 & 4,53 & 7,46 & 0,0099 \\
\hline$A C$ & 26,43 & 1 & 26,43 & 43,49 & $<0.0001$ \\
\hline$A^{2}$ & 34,76 & 1 & 34,76 & 57,20 & $<0.0001$ \\
\hline$A^{2} C$ & 15,97 & 1 & 15,97 & 26,29 & $<0.0001$ \\
\hline Residual & 20,66 & 34 & 0,61 & & \\
\hline Lack of Fit & 17,59 & 24 & 0,73 & 2,39 & 0,0767 \\
\hline Pure Error & 3,07 & 10 & 0,31 & & \\
\hline
\end{tabular}

ANOVA-MODEL: RISSANFÄLLIGKEIT IN ABHÄNGIGKEIT VON PRESSDAUER UND PRESSTEMPERATUR

Für das schnell härtende Harz verpresst mit vorgeheiztem Pressblech:

\begin{tabular}{llllll} 
Source & Sum of & & Mean & $\mathbf{F}$ & P-value \\
\hline Model & Squares & df & Square & Value & Prob > F \\
\hline A-Dauer & $2,361 \mathrm{E}+006$ & 3 & $7,871 \mathrm{E}+005$ & 14,69 & 0,0013 \\
B-Temperatur & $1,581 \mathrm{E}+006$ & 1 & $1,581 \mathrm{E}+006$ & 29,51 & 0,0006 \\
AB & $7,511 E+005$ & 1 & $7,511 E+005$ & 14,02 & 0,0057 \\
\hline Residual & $3,570 E+005$ & 1 & $3,570 E+005$ & 6,66 & 0,0326 \\
\hline \hline Cor Total & $4,286 \mathrm{E}+005$ & 8 & 53579,33 & & \\
\hline
\end{tabular}


Für das schnell härtende Harz verpresst mit kaltem Pressblech:

\begin{tabular}{llllll} 
Source & $\begin{array}{l}\text { Sum of } \\
\text { Squares }\end{array}$ & df & Square & Value & Prob > F \\
\hline \hline Model & $1,731 \mathrm{E}-003$ & 4 & $4,327 \mathrm{E}-004$ & 28,06 & 0,0035 \\
\hline A-Zeit & $4,949 E-004$ & 2 & $2,475 E-004$ & 16,05 & 0,0123 \\
B-Temperatur & $1,236 \mathrm{E}-003$ & 2 & $6,180 \mathrm{E}-004$ & 40,07 & 0,0023 \\
\hline Residual & $6,169 \mathrm{E}-005$ & 4 & $1,542 \mathrm{E}-005$ & & \\
\hline \hline Cor Total & $1,793 \mathrm{E}-003$ & 8 & & &
\end{tabular}

Für das langsam härtende Harz verpresst mit kaltem Pressblech:

\begin{tabular}{|c|c|c|c|c|c|}
\hline \multirow[b]{2}{*}{ Source } & \multicolumn{2}{|l|}{ Sum of } & \multirow{2}{*}{$\begin{array}{l}\text { Mean } \\
\text { Square }\end{array}$} & \multirow{2}{*}{$\begin{array}{l}\text { F } \\
\text { Value }\end{array}$} & \multirow{2}{*}{$\begin{array}{l}\text { P-value } \\
\text { Prob > F }\end{array}$} \\
\hline & Squares & $d f$ & & & \\
\hline Model & 153,78 & 4 & 38,44 & 6,47 & 0,0490 \\
\hline$A-Z e i t$ & 22,89 & 2 & 11,44 & 1,93 & 0,2596 \\
\hline B-Temperatur & 130,89 & 2 & 65,44 & 11,01 & 0,0236 \\
\hline Residual & 23,78 & 4 & 5,94 & & \\
\hline Cor Total & 177,56 & 8 & & & \\
\hline
\end{tabular}

ANOVA-MODEL: BIEGEFESTIGKEIT IN ABHÄNGIGKEIT VON PRESSDAUER UND PRESSTEMPERATUR

ANOVA Model zur Auswertung der Biegefestigkeit mit den Parametern Pressdauer (A) und Presstemperatur (B)

\begin{tabular}{|c|c|c|c|c|c|}
\hline Source & $\begin{array}{l}\text { Sum of } \\
\text { Squares }\end{array}$ & $d f$ & $\begin{array}{r}\text { Mean } \\
\text { Square }\end{array}$ & F-value & p-value \\
\hline Model & 143,03 & 5 & 28,61 & 38,79 & $<0.0001$ \\
\hline A-Pressdauer & 28,58 & 1 & 28,58 & 38,75 & $<0.0001$ \\
\hline B-Presstemp. & 75,67 & 1 & 75,67 & 102,60 & $<0.0001$ \\
\hline$A B$ & 35,29 & 1 & 35,29 & 47,85 & $<0.0001$ \\
\hline$B^{2}$ & 0,18 & 1 & 0,18 & 0,24 & 0,6251 \\
\hline$A B^{2}$ & 10,99 & 1 & 10,99 & 14,90 & 0,0003 \\
\hline Residual & 35,40 & 48 & 0,74 & & \\
\hline Lack of Fit & 1,21 & 3 & 0,40 & 0,53 & 0,6634 \\
\hline Pure Error & 34,19 & 45 & 0,76 & & \\
\hline
\end{tabular}


ANOVA-MODEL: MIKRORISSDICHTE IN ABHÄNGIGKEIT VON PRESSDAUER UND PRESSTEMPERATUR

ANOVA Model zur Auswertung der Mikrorissdichte mit den Parametern Pressdauer (A) und Presstemperatur (B)

\begin{tabular}{|c|c|c|c|c|c|}
\hline Source & $\begin{array}{r}\text { Sum of } \\
\text { Squares }\end{array}$ & df & $\begin{array}{r}\text { Mean } \\
\text { Square }\end{array}$ & F-value & p-value \\
\hline Model & 67252 & 5 & 13450 & 67,66 & $<0.0001$ \\
\hline A-Pressdauer & 4298 & 1 & 4298 & 21,62 & $<0.0001$ \\
\hline B-Presstemp. & 30310 & 1 & 30310 & 152,47 & $<0.0001$ \\
\hline$A B$ & 5676 & 1 & 5676 & 28,55 & $<0.0001$ \\
\hline$B^{2}$ & 3305 & 1 & 3305 & 16,63 & 0,0002 \\
\hline$A B^{2}$ & 1244 & 1 & 1244 & 6,26 & 0,0167 \\
\hline Residual & 7753 & 39 & 199 & & \\
\hline Lack of Fit & 14 & 3 & 5 & 0,02 & 0,9958 \\
\hline Pure Error & 7739 & 36 & 215 & & \\
\hline
\end{tabular}

